


\section{Propositions}

1. Quantitative and qualitative resistance should be defined based on their phenotypic segregation rather than on the efficacy of their defense responses. (this thesis)

2. A reduction in foliar symptoms on tomato does not always imply increased resistance against $V$. dahliae.

(this thesis)

3. The proportion of papers in scientific journals which describe negative results must increase significantly.

4. Just like a book shouldn't be judged by its cover, speakers at scientific conferences shouldn't be judged by their abstracts.

5. If left unchecked, artificial intelligence will become one of the greatest threats to society.

6. True leaders don't dominate, but motivate.

Propositions belonging to the thesis, entitled

Identification and inheritance of quantitative resistance against

Verticillium dahliae in tomato

Jasper Pepijn Vermeulen

Wageningen, 20 October 2020 


\section{Identification and inheritance of quantitative resistance against Verticillium dahliae in tomato}




\section{Thesis committee}

\section{Promotors}

Prof. Dr Yuling Bai

Personal chair, Plant Breeding

Wageningen University \& Research

Prof. Dr Bart P.H.J. Thomma

Professor of Phytopathology

Wageningen University \& Research

\section{Co-promotor}

Dr Henk J. Schouten

Researcher, Plant Breeding

Wageningen University \& Research

\section{Other members}

Prof. Dr M.G.M. Aarts, Wageningen University \& Research

Dr P.M. Bleecker, Universiteit van Amsterdam

Prof. Dr A.F.J.M. van den Ackerveken, Utrecht University

Dr J.C. Boshoven, Bejo Zaden, Warmenhuizen

This research was conducted under the auspices of the Graduate School Experimental Plant Sciences (EPS) 


\title{
Identification and inheritance of quantitative resistance against Verticillium dahliae in tomato
}

\author{
Jasper Pepijn Vermeulen
}

\section{Thesis}

submitted in fulfilment of the requirements for the degree of doctor

at Wageningen University

by the authority of the Rector Magnificus,

Prof. Dr A.P.J. Mol,

in the presence of the

Thesis Committee appointed by the Academic Board

to be defended in public

on Tuesday 20 October 2020

at 4 p.m. in the Aula 
Jasper P. Vermeulen

Identification and inheritance of quantitative resistance against Verticillium dahliae in tomato, 184 pages.

$\mathrm{PhD}$ thesis, Wageningen University, Wageningen, the Netherlands (2020)

With references, with summary in English.

ISBN: 978-94-6395-513-3

DOI: https://doi.org/10.18174/529717 


\section{Table of contents}

$\begin{array}{lll}\text { Chapter } 1 & \text { General Introduction } & 7\end{array}$

Chapter 2 Evaluation of phenotyping and inoculation procedures for the identification of enhanced resistance against Verticillium dahliae in tomato

Chapter 3 Screening of wild tomato germplasm reveals broad-spectrum resistance against Verticillium wilt disease in at least two accessions

Chapter 4 Mapping of Verticillium dahliae race 2 resistance reveals multiple QTLs from susceptible Solanum lycopersicum Moneymaker, but not from resistant Solanum pimpinellifolium VG-3 and VG-21

Chapter 5 At least three QTLs are associated with the resistance of Solanum cheesmanii VG-20 to a race 2 strain of Verticillium dahliae

Chapter 6 Comparative genomics reveals two candidates for the Verticillium dahliae in planta-secreted effector protein recognized in $\mathrm{V} 2$ tomato plants

Chapter 7 General discussion

References

$\begin{array}{ll}\text { Summary } & 171\end{array}$

$\begin{array}{ll}\text { Aknowledgements } & 175\end{array}$

$\begin{array}{ll}\text { About the author } & 179\end{array}$

$\begin{array}{lr}\text { Education statement } & 181\end{array}$ 



\section{Chapter 1}

\section{General Introduction}

Jasper P. Vermeulen ${ }^{1,2}$

'Laboratory of Phytopathology, Wageningen University \& Research, 6708 PB Wageningen, The Netherlands. ${ }^{2}$ Laboratory of Plant Breeding, Wageningen University \& Research, 6708 PB Wageningen, The Netherlands. 


\section{Introduction}

Plants have established symbiotic relationships with microorganisms for hundreds of millions of years, most likely already before they started to colonize the land (Han 2019). After all, the origin of plants is thought to be the result of the integration of a cyanobacterium-like prokaryote in the common ancestors of plants (Mereschkowsky 1905; Gould et al. 2008). Despite that many present-day symbiotic relationships between plants and microorganisms have no detrimental or even beneficial effects for the plant, some microorganisms are pathogenic and cause disease. Land plants host a great diversity of pathogens, including fungi, bacteria, oomycetes, and viruses. Although the precise origin of these antagonistic relationships of plants with microorganisms is difficult to determine, it is safe to say that fungi, bacteria, oomycetes and viruses have probably been infecting plants for hundreds of millions of years (Han 2019). Since the first crops were domesticated at the dawn of agriculture over 10.000 years ago (Focus 2007), farmers have been continuously challenged by plant pathogens. In fact, the domestication of crops and its cultivation in large homogeneous monocultures has had a significant effect on the evolution of these pathogens, likely resulting in more aggressive pathogens which are highly specialized to crops grown in agricultural production systems (Stukenbrock and McDonald 2008). Although no one knows exactly how much food and feed is lost on due to diseases caused by microbial pathogens, available estimates indicate that between $10 \%$ and $30 \%$ of the worlds harvestable yields are lost due to such diseases (Oerke 1999, 2006; Flood 2010). To control pathogen damage, one of the main approaches has been to employ chemical control agents such as fungicides, oomycides and bactericides to control fungal, oomycete and bacterial pathogens, respectively. The control of plant pathogens with these chemicals has contributed to the steady increase in crop yields over the last half-century (Cooper and Dobson 2007; Oerke 2006). However, more recently, the use of chemical control in agriculture has become severely restricted, particularly in Europe (Lamichhane et al. 2016), and an increasing number of plant pathogens has developed resistance against particular agrochemicals (Hollomon 2015; Network 2016). Therefore, effective alternative strategies are desirable to protect crops against pathogens. A popular alternative strategy to control pathogens is to improve the natural defences of plants against pathogens, mostly by breeding more resistant crops.

\section{How plants cope with pathogens: tolerance and resistance}

In the hundreds of millions of years of engaging in antagonistic relationships with pathogens, plants have evolved various mechanisms to cope with pathogens. Broadly speaking, these mechanisms can be divided into tolerance and resistance. Tolerance generally refers to mechanisms of the plant which reduce the damage of the pathogen infection without 
restricting pathogen colonization. In agricultural settings, the term tolerance is also used to refer to mechanisms which enable the plant to endure pathogen infection with minimal impact on yield. Resistance refers to mechanisms which reduce or completely halt pathogen colonization on the host, which results into a reduction in the damage of the infection on the host in turn.

\section{Tolerance: tolerating the presence of the pathogen}

Tolerance has been described in multiple plant species against various pathogen species, including fungal pathogens (Dan et al. 2001; Politowski 1978; Roberts 1984; Mikaberidze and McDonald 2020), bacterial pathogens (Buell and Somerville 1995), oomycete pathogens (Salvaudon and Shykoff 2013) and viral pathogens (Hily et al. 2016). Several mechanisms have been suggested to play a role in tolerance to plant pathogens. Firstly, plants can compensate for the reduced photosynthesis activity in infected plant parts by increasing the $\mathrm{CO}_{2}$ fixation (Inglese and Paul 2006) or by induction of photosynthetic light-reaction components (Stare et al. 2015) in non-infected tissues. Secondly, tolerance mechanisms have been reported to reallocate resources from growth into reproduction (Pagán et al. 2008; Veronese et al. 2003). Finally, it has been suggested that upon pathogen infection, low levels of defence responses can, in some cases, repress symptom development without affecting pathogen colonization (Chamnongpol et al. 1998; Alexander et al. 1993; Block et al. 2005). When compared with resistance, tolerance has received relatively little attention from breeders, which may be related to the fact that tolerance does not control the pathogen, but only the disease. After all, even though tolerant plants may display similar yields as healthy plants, inoculum accumulation still takes place. Similarly, tolerance mechanisms which reduce fitness loss do not always reduce yield loss. For example, mechanisms which cause resource allocation from growth to reproduction may result in yield losses in crops of which non-reproductive plant parts are harvested. Still, because of the phenotypes of tolerance and resistance that are sometimes similar, namely a reduction in symptom expression, the two terms are occasionally confused. For example, it has been demonstrated that resistance against the fungal vascular pathogen Verticillium dahliae cannot be fully distinguished from tolerance without quantifying the pathogen biomass in infected plants (Dan et al. 2001). In some cases, breeders may even have inadvertently selected for tolerant genotypes rather than resistant genotypes. For example, by solely focussing on symptom development, breeders may have selected wheat genotypes which are tolerant, but not resistant to Zymoseptoria tritici (Mikaberidze and McDonald 2020).

\section{Qualitative R-gene resistance against plant pathogens}

In contrast to tolerance, resistance restricts the colonization of the pathogen, and thereby reduces the disease. The most well-studied type of resistance is qualitative resistance, which segregates into discrete classes of resistant and susceptible genotypes and is in many cases 
based on single dominant resistance $(R)$ genes. Because of the relatively simple genetics of qualitative resistance, $R$ genes have been used in resistance breeding since the beginning of the $20^{\text {th }}$ century. For example, by 1934, $R$ gene resistance was already described in 13 different crops against 23 different diseases (Hansen 1934; Allard 1960). In the second half of the $20^{\text {th }}$ century, phytopathologists began unravelling the dynamics behind $R$ gene resistance. In the 1940s, Harald Flor introduced the gene-for-gene hypothesis based on his observations of the interaction between leaf rust (Melampsora lini) and flax (Linum usitatssimum). Flor's gene-for-gene hypothesis states that for each dominantly inherited $R$ gene in a host plant, there is a corresponding dominantly inherited avirulence (Avr) gene in the pathogen. The product of the $R$ gene recognizes the product of the corresponding Avr gene, resulting in resistance. When the pathogen loses the avirulence gene or possesses a variant of which the product is no longer recognized by the product of the $R$ gene, this results in susceptibility.

In 2006, Dangl and Jones presented the famous "zigzag model" to explain the evolutionary arms race between plants and pathogens (Jones and Dangl 2006). According to this model, the first layer of the plant's immune system against pathogens is governed by Pattern Recognition Receptors (PRRs), which recognize conserved molecular patterns of the pathogen, referred to as Microbe Associated Molecular Pattern (MAMPs), or Pathogen Associated Molecular Patterns (PAMPs). MAMPs are broadly conserved among microbial classes and contribute to microbial fitness. Recognition of MAMPs by PRRs triggers MAMP-triggered immunity (MTI), also referred to as PAMP-triggered immunity (PTI). To overcome MTI, pathogens have evolved specific effector molecules that interfere with MTI, leading to Effector-Triggered Susceptibility (ETS). In contrast to MAMPs, effectors are specific to a single or few related species and not broadly conserved among microbial classes. They generally contribute to pathogen virulence. To cope with ETS and reinstall immunity, plants evolved a second layer of defence, which is governed by specialized resistance $(\mathrm{R})$ proteins that recognize effectors, either directly or indirectly, resulting in Effector-Triggered Immunity (ETI; Jones and Dangl 2006). When compared with MTI, ETI is generally considered to be a stronger defence response.

Although the zigzag model is useful to explain the evolutionary arms race between plants and pathogens, accumulating molecular evidence has indicated that the binary distinction between PRRs and R proteins, as well as between the corresponding MAMPs and effectors, is oversimplified as in reality there is a continuum between these molecules (Thomma et al. 2011; Cook et al. 2015). Consequently, not all microbial compounds which trigger immunity can be classified either as MAMPs or as effectors. For example, some effectors are not strainor species-specific but are widely distributed, such as fungal LysM effectors (de Jonge and Thomma 2009) and conserved Nep1-like proteins (NLPs) Cook et al. 2015; Thomma et al. 2011). Similarly, some MAMPs may also be required for virulence, such as flagellin, which contributes to the virulence of bacterial pathogens such as Pseudomonas syringae pv. Tabaci 
(Taguchi et al. 2010, 2006). Besides for the detection of MAMPs and effectors, plants also possess immune receptors to respond to infection-associated molecules that are generated by damage of the infection, termed as Damage Associated Molecular Patterns (DAMPs), which are not included in the zigzag model (Boller and Felix 2009). These arguments justified an update of the zigzag model.

Recently, a new model, termed the "invasion model" was proposed (Cook et al. 2015; Thomma et al. 2011), which accommodates all possible molecular elicitors of immune responses, such as MAMPs, DAMPs and effectors, as 'invasion patterns' or 'IPs' which are then recognized by "invasion pattern receptors" or 'IPRs'. Recognition of IPs by IPRs triggers IP-triggered responses (IPTRs) that may result into successful defence, leading to resistance. However, this model also appreciates that particular pathogens activate immune responses in the host that lead to the actual stimulation of disease, such as necrotrophic pathogens that benefit from host cell death. A slightly more extensive model, the 'spatial immunity model' was proposed by van der Burgh et al. (2019), which incorporates where immune receptors detect infection-associated molecules. According to this model, cell-surface receptors such as receptor like proteins (RLPs) and receptor like kinases (RLKs) recognize so-called "extracellular immunogenic patterns" (ExIPs) which results in "extracellularly triggered immunity" (ExTI). Accordingly, cytoplasmatic immune receptors, such as NB-LRRs, recognize "intracellular immunogenic patterns" (InIPs), which triggers "intracellularly triggered immunity" (InTI).

Over the last century, over $300 R$ genes have been cloned and characterized (Kourelis and Van Der Hoorn 2018). The majority of these encode cytoplasmatic immune receptors, such as nucleotide binding site-leucine rich repeat receptors (NB-LRRs) which, following the spatial immunity model, thus trigger InTI. The second major class of the cloned $R$ genes encode cell-surface receptors, including RLPs and RLKs, which trigger ExTI. Although the defence responses triggered by the R proteins encoded by $R$ genes, especially InTI triggered by NB-LRRs, often results into complete immunity, $R$ gene-mediated resistance is infamous for its lack of durability. $R$ genes are often relatively quickly overcome by pathogens, causing breeders to need to continuously search for additional resistance sources (St.Clair 2010). The high level of resistance provided by many $R$ genes can cause a strong diversifying selection on pathogen populations to select for pathogen variants which are no longer recognized by the $R$ gene, or for pathogen variants which suppress the resistance response in plants (Brown 2015). However, the strength of this diversifying selection mainly depends on the fitness costs of losing the avirulence factor that is recognized by the R gene (Brown 2015), which can explain why some $R$ genes are more durable than others. 


\section{Susceptibility genes}

In some cases, qualitative resistance inherits recessively. In these cases, the resistance is not governed by an $R$ gene, but by an impaired susceptibility $(S)$ gene. In contrast to $R$ genes, $S$ genes facilitate susceptibility of the host to a pathogen. As reviewed by van Schie and Takken (2014), three types of $S$ genes are currently recognized. Firstly, the products of some $S$ genes facilitate the recognition and penetration of the host by the pathogen. Secondly, other $S$ gene products act as negative regulators of immune signalling in the plant. Finally, the products of some $S$ genes fulfil metabolic needs of the pathogen in the plant. Impairment of $S$ genes can result in a reduced colonization by the pathogen, leading to increased resistance. In contrast to typical $R$ gene-mediated resistance, disabling of $S$ genes can result in relatively broad-spectrum and more durable resistance (Pavan et al. 2010; van Schie and Takken 2014). However, although the products of identified $S$ genes thus facilitate disease, they have not been lost during evolution, likely because they also have important intrinsic functions for the plant. Although impairment of $S$ genes can thus lead to increased resistance, this may also cause pleiotropic effects which may result in decreased plant fitness or crop yields (Pavan et al. 2010; van Schie and Takken 2014).

\section{Quantitative resistance}

When the first $R$ genes were discovered at the beginning of the $20^{\text {th }}$ century, some researchers also reported resistance which was not governed by a single $R$ gene, but by multiple genetic loci. By 1934, a total of 88 publications reported resistances in 15 different crops against 29 different diseases which were based on multiple genes (Hansen 1934; Allard 1960). Like other biological traits, resistance can be classified as either quantitative or qualitative. Whereas qualitative resistance produces distinct classes of resistant and susceptible individuals and segregates as simple mendelian loci, quantitative resistance produces a continuous range of phenotypes ranging from susceptible to resistant (Corwin and Kliebenstein 2017). Following this definition, resistance based on single $R$ genes or impaired $S$ genes can generally be categorized as qualitative resistance, whereas resistance based on multiple loci can in most cases be categorized as quantitative resistance. However, the term quantitative resistance has also been used to refer to partial resistance (Cowger and Brown 2019; Niks et al. 2015). Still, in this thesis, I will use the terms quantitative and qualitative resistance to refer to the phenotypic segregation of the resistance, not the efficacy of its defence responses. According to this criterion, quantitative resistance thus refers to any form of resistance that produces a continuous distribution from susceptible to resistant phenotypes and is therefore generally not based on a single $R$ - or $S$-gene, but on multiple, often small-effect loci.

In contrast to qualitative resistance, quantitative resistance is still relatively poorly understood. Before the advent of molecular markers, unravelling genetically complex traits was very difficult, as genetic maps were commonly not sufficiently dense to map genetic traits (Lander and Botstein 1989). After the emergence of the first molecular DNA markers 
in the 1980s, the genetics of quantitative traits slowly began to be unravelled. The enhanced genetic maps combined with the emergence of statistical methods allowed researchers to analyse the relationship between marker genotypes and phenotypic variation through analyses such as interval mapping, introduced by Lander and Botstein in 1989. With these methods, chromosomal regions associated with quantitative traits, also referred to as "quantitative trait loci" (QTLs), could be identified.

By cloning of the causal genes of QTLs, the diverse mechanistic bases underlying quantitative resistance are slowly being unravelled. Although the actual cloning of causal genes from resistance QTLs is still rather rare, the causal genes that have been identified appear to be involved in diverse mechanisms. First of all, multiple quantitative resistance genes have been shown to be involved in pathogen detection. For example, of the major QTLs identified for the quantitative disease resistance in rice against Magnaporthe oryzae, the majority of the cloned loci encoded either RLKs or NB-LRRs (Raboin et al. 2016; Ballini et al. 2008; Miah et al. 2013; Kang et al. 2016). Besides pathogen perception, several quantitative resistance genes have been shown to be involved in defence signal transduction downstream of pathogen perception. For example, cloned genes were found to encode defensins, pathogenesisrelated proteins, and several genes were found to be involved in PAMP signalling (Kump et al. 2011; Belcher et al. 2012). Thirdly, quantitative resistance genes may be involved in chemical warfare in plant-pathogen interactions (Poland et al. 2009). Finally, some evidence suggests that some quantitative resistance genes may be involved in the regulation of developmental and morphological phenotypes in the plants which may have pleiotropic effects on disease resistance. For example, traits such as stomatal aperture and/or density, or the plant's ability to repel water have been suggested to also affect immunity against particular foliar pathogens (Melotto et al. 2006; Bradley et al. 2003). Although more light has thus been shed on potential mechanisms for quantitative disease resistance, the identified QTLs and causal genes may only be the tip of the iceberg. As mapping populations are typically limited in size, and therefore limited in power to detect a high number of small-effect loci, this results into an underestimation of the true number of quantitative resistance loci, especially when resistances are highly polygenic (Corwin and Kliebenstein 2017). It appears that in contrast to qualitative resistance, which is typically governed by $R$ genes, there is no single unifying mechanism that underlies qualitative resistance, which partially explains why quantitative resistance is still relatively poorly understood.

Despite the genetic complexity of quantitative resistance, interest in this type of resistance has grown over the last decades. A common justification for studying quantitative resistance is its potential durability and broad-spectrum efficacy. By comparing various forms of resistance, Parlevliet (2002) concluded that quantitative resistance generally appears to be more durable than $R$ gene resistance. Theoretically, this is not very surprising. Because of the polygenic nature of quantitative resistance, pathogen variants that overcome a single 
gene that contributes partially to the quantitative resistance may experience a smaller evolutionary advantage than pathogen variants that overcome a qualitative $R$ gene. Therefore, pathogen variants which overcome a partial quantitative resistance gene likely spread less rapidly through the pathogen population than strains which have overcome a major $R$ gene which governs a strong resistance response, and thus provides a stronger selection pressure. However, some examples demonstrate that quantitative resistance is not always durable or broad-spectrum. For example, multiple QTL mapping studies for resistance against $B$. cinerea revealed that the mapped QTLs do typically not have broadspectrum efficacy, and that the effect of the QTLs were largely dependent on the pathogen genotype (Corwin and Kliebenstein 2017), which was also found for QTLs for resistance against S. sclerotiorum in sunflower (Davar et al. 2011). Thus, it remains to be determined how individual loci for quantitative resistance behave with respect to broad-spectrum efficacy and durability of the resistance.

\section{The Tomato: From a werewolves' snack to major vegetable crop}

According to old legends from pre-Columbian America, the tomato was initially known as a fruit eaten by werewolves and wolves. This is reflected in the old name of the tomato, Lycopersicon esculentum, which means edible (esculentum) wolf peach (lyco-persicon) (Preedy and Watson 2019). Although Linneaus treated the tomato as a member of the genus Solanum already in 1753, little consensus existed for a long time whether tomato should indeed be part of the genus Solanum, or whether it should be treated as a distinct Lycopersicon genus. Only when DNA sequencing technology accelerated phylogenetic analyses, it became widely accepted that the tomato is deeply nested in the genus Solanum, and should be renamed as Solanum lycopersicum (Liedl et al. 2013; Spooner et al. 1993; Asamizu and Ezura 2009).

From an evolutionary point of view, as a 12 million years old clade, the tomato clade is relatively young (Wikström et al. 2001). Around 7 million years ago, its radiation began across western South America (Clint Nesbitt and Tanksley 2002). The precise origin of the domesticated tomato is still under debate, as it has been hypothesized to either originate from the Andean region or from Mesoamerica. However, Blanca et al. (2012) reported evidence which suggests that pre-domestication of the tomato took place in the Andean region, and that the domestication of the tomato was then subsequently completed in Mesoamerica. Likely, the name "tomato" originates from Mesoamerican region, as the Aztecs named the fruit "tomatl", meaning "plants bearing globous and juicy fruit" (Bauchet and Causse 2012). After conquering the Aztec city Tenochtitlan in 1521, known as Mexico city today, the Spanish conquistador Cortes likely introduced the tomato into Spain, after which it spread further throughout Europe and the rest of the world (Blanca et al. 2012; Bai and Lindhout 2007; Bergougnoux 2014). Due to its resemblance with toxic Solanum 
species, the tomato was long used only for ornamental purposes. Only from the late $17^{\text {th }}$ century onwards, the tomato was incorporated into the local cuisine in southern European countries such as Italy. However, the domestication of the tomato as an edible vegetable only really accelerated in the $19^{\text {th }}$ century (Bergougnoux 2014). By the end of the $19^{\text {th }}$ century, various tomato cultivars were available in different colours and for different purposes (Bai and Lindhout 2007). From the beginning of the $20^{\text {th }}$ century onwards, public institutes became more involved in breeding, and private breeding companies shifted towards hybrids. Breeding of tomatoes went through multiple phases; from breeding for yield in the 1970s, shelf-life in the 1980s, breeding for taste in the 1990s, and currently for nutritional quality (Bai and Lindhout 2007; Schouten et al. 2019). Simultaneously, breeders sought for resistance against pests and pathogens, which intensified in the 1970s due to increased pressure to limit the usage of chemical control agents. Although the genetic diversity among tomato cultivars was low in the 1960s, Schouten et al. (2019) demonstrated that the genetic diversity in the tomato cultivars increased substantially since the 1970s in north-western Europe, likely due to the breeding for resistance and taste.

Currently, the tomato is, after potato, the most produced vegetable crop in the world (FAO 2020) Although the global average yields per hectare of tomato production systems have tripled over the last half-century (FAO 2020), several challenges for tomato production remain. Because the tomato is susceptible to over 200 pests and pathogens (Jones 1991), one of the main remaining challenges is disease management. Plant pathogens cause a potential average yield loss on tomato of over 19\% (Zalom 2003), making breeding for resistance valuable to further improve tomato production. Up to this date, over 60 tomato genes that are involved in interactions with various insects and pathogens have been mapped, including both $R$ genes, $S$ genes and quantitative resistance loci (Bai and Lindhout 2008). In this thesis, the main focus is on the identification of genes or QTLs for resistance against vascular wilt disease caused by the filamentous fungal pathogen Verticillium dahliae.

\section{Verticillium wilt disease}

Verticillium wilt is a vascular disease caused by multiple species of ascomycete fungal genus Verticillium. First mentioned by Nees von Essenbeck in 1817, Verticillium is a relatively small genus that currently consists of ten soil-borne species (Essenbeck 1817; Inderbitzin et al. 2011). Of the ten Verticillium species, V. isaaci, V. klebahnii, V. nubilum, V. tricorpus and $V$. zageramsianum have a mostly saprophytic lifestyle and only on rare occasions cause opportunistic infections in plants. The other five species, V. albo-atrum, V. alfalfa, V. dahliae, $V$. longisporum and $V$. nonalfalfa are all plant pathogens that cause plant diseases. Although all five pathogenic species cause damage on various crops, $V$. dahliae is economically the most important pathogen. First isolated from dahlia plants and identified as a new species 
by Klebahn in 1913, this notorious pathogen causes disease on over 200 plant species, including crops such as tomato, potato, eggplant, pepper, olive, strawberry, sunflower and cotton. Besides its enormous host range, $V$. dahliae produces resting structures, called microsclerotia, which can remain viable in the soil for many years (Wilhelm 1955), making crop rotation difficult. $V$. dahliae is a monocyclic pathogen, meaning that one cycle of infection and inoculum production occurs within one growing cycle (Fradin and Thomma 2006). The life cycle of $V$. dahliae starts when hyphae grow out of microsclerotia, the germination of which is likely triggered by excess carbon and nitrogen originating from exudates released in the rhizosphere (Grayston et al. 1997). Possibly directed by nutrient gradients, the hyphae grow towards the roots of the host, and typically infect susceptible plants by entering the roots through wounds at the root tips or at sites of lateral root formation (Bishop and Cooper 1983). Once in the roots, the hyphae enter the xylem vessels where they produce conidiospores that are carried upwards through the xylem vessels with the sap stream (Fradin and Thomma 2006). When conidiospores get trapped in pit cavities or xylem vessel end walls, the conidiospores germinate and hyphae invade adjacent xylem vessels to continue the colonization (Bishop and Cooper 1983; Fradin and Thomma 2006). By the time the shoots and roots have become fully colonized by $V$. dahliae, the fungus enters its saprophytic stage, and necrosis and senescence of the plant tissue begins (Fradin and Thomma 2006). At the end of the disease cycle, $V$. dahliae produces large amounts of microsclerotia which are released into the soil when the dead plant tissue decomposes.

Symptoms of Verticillium wilt depend on the host species and the environment (Fradin and Thomma 2006). On tomato, symptoms may include wilting, yellowing and necrosis of leaves, as well as stunting (Fig. 1). Although no real consensus exists about the primary cause of the Verticillium wilt disease symptoms, several mechanisms have been suggested to be involved in symptom development (Fradin and Thomma 2006; Pegg and Brady 2002). First of all, $V$. dahliae produces various phytotoxins and cell-death elicitors which have been suggested to cause chlorosis, necrosis and possibly wilting. Besides phytotoxins, V. dahliae produces cell-wall degrading enzymes such as pectinolytic enzymes that may contribute to necrosis and wilting. Thirdly, vessel occlusion, either caused by physical blockage by the pathogen or by host defence responses have been reported to cause wilting symptoms. Finally, stunting of infected plants may also be caused by reduced photosynthesis rates upon stomatal closure due to water stress (Fradin and Thomma 2006; Pegg and Brady 2002).

As a soil-borne pathogen, $V$. dahliae is especially problematic in soil-based production systems, and to a much lesser extent in substrate-based production systems that use alternative growing media such as rockwool (Ownley and Trigiano 2017), as for instance commonly used in Dutch tomato greenhouses. Yield losses caused by $V$. dahliae commonly range from $10 \%$ to $15 \%$, but losses over $50 \%$ have been reported for multiple economically valuable crops such as potato, cotton, strawberry and lettuce (Dean et al. 2014; Pegg and Brady 2002; Klosterman et al. 2009). Because the pathogen resides inside the plant for 
most of the disease cycle, most fungicides are ineffective against $V$. dahliae. Combined with its large host-range and the longevity of microsclerotia (Wilhelm 1955), this makes that Verticillium wilt is difficult to control. $V$. dahliae generally spreads through the transfer of contaminated soil, contaminated equipment, irrigation and infected plant materials such as rootstocks and tubers (Fradin and Thomma 2006). In warmer climates, solarization of the soil can reduce, or even eliminate the inoculum in the soil. Chemical methods such as soil fumigations also reduce the inoculum in the soil, but due to environmental and public health concerns, this practice has become banned in most parts of the world (Fradin and Thomma 2006). Another approach to control Verticillium wilts is the use of the natural defence system of the plant by employing genetic resistance.

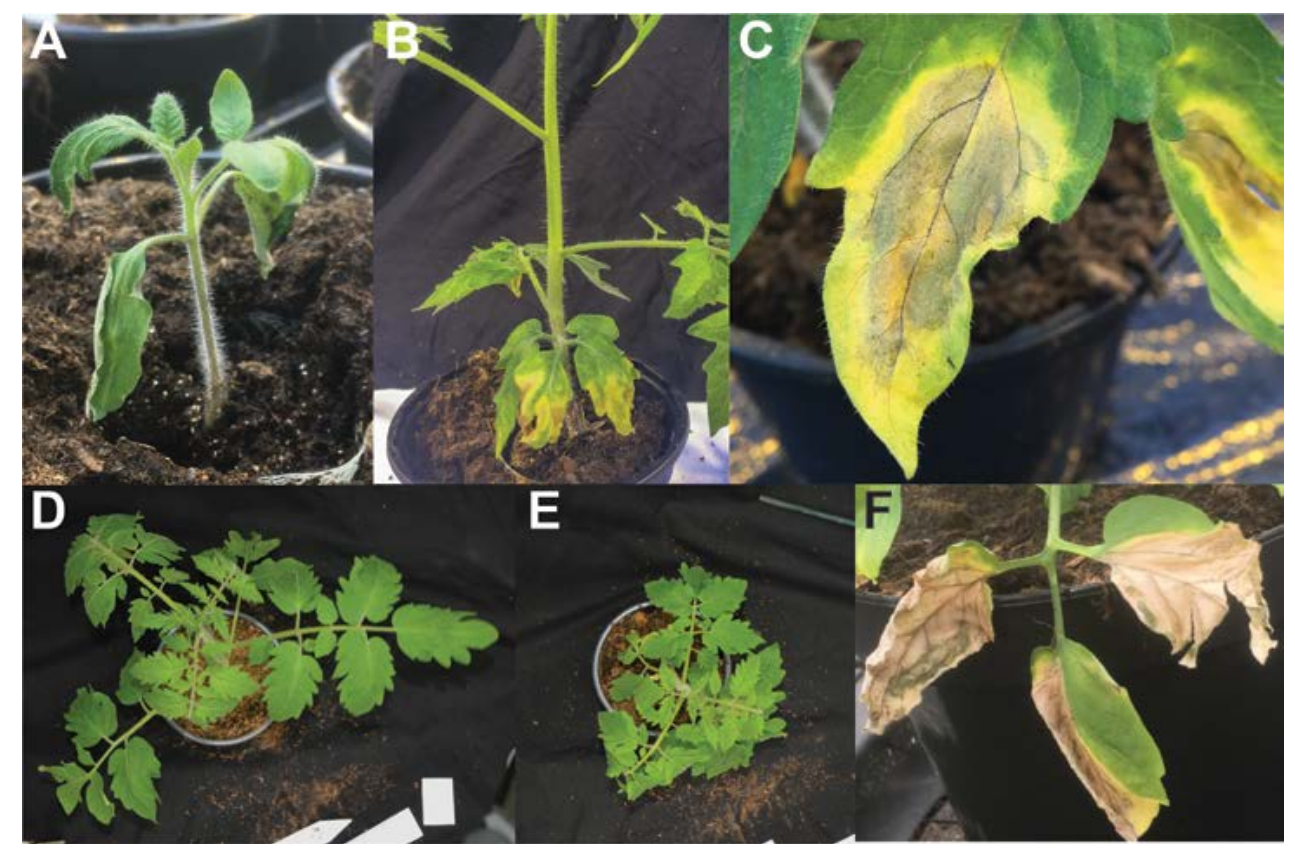

FIGURE1 | Verticillium wilt disease symptoms on tomato plants at 10 (A), 21 (B-E), and 32 (F) days post inoculation with V. dahliae. (A) displays wilting, (B), (C) and (F) display yellowing and necrosis of leaves. (E) displays the stunting of an infected plant when compared with a mock-inoculated plant (D).

In resistant plants, the defence against $V$. dahliae has two phases, the pre-vascular and postvascular phase (Fradin and Thomma 2006). Before entering the xylem vessels, V. dahliae needs to cross the endodermis of the root cortex, which acts as a natural barrier against microbial infection. In many infections, $V$. dahliae does not cross the endodermis, and remains trapped in the outer layers of the root (Talboys 1972; Huisman 1988). When hyphae from $V$. dahliae manage to cross the endodermis and enter the xylem, the post-vascular phase of the infection begins. In this phase, plants employ both physical and chemical defence responses to contain the pathogen. Physical defence responses commonly result in 
the occlusion of xylem vessels by gums, gels and tyloses, which block the movement of the fungus (Yadeta et al. 2013). Further spread of the fungus to adjacent xylem vessels is also limited by coating and reinforcements of the vascular cell walls. Although these defence responses occur both in susceptible plants and resistant plants, the defence responses are thought to occur faster and more extensively in resistant plants (Fradin and Thomma 2006; Yadeta et al. 2013). Besides these physical defence mechanisms, chemical defence also plays a major role in the defence against $V$. dahliae in the xylem. Part of the chemical defence is aimed at modulating the morphology of the xylem tissue to inhibit fungal colonization (Yadeta et al. 2013). Other compounds have antimicrobial activity and eliminate the trapped fungus (Fradin and Thomma 2006; Yadeta et al. 2013). This elimination occurs both in susceptible and resistant plants. However, whereas in susceptible plants the fungus recovers and begins to spread further through the xylem, the fungus does not overcome this elimination in resistant plants (Gold and Robb 1995).

\section{Breeding for resistance against $V$. dahliae in tomato}

After beginning to cause severe damage on crops in Europe and the US in the late $19^{\text {th }}$ and early $20^{\text {th }}$ century, $V$. dahliae caught the attention of breeders and phytopathologists (van der Lek 1919; Rudolph 1931). Already at that time, the employment of genetic resistance was mentioned as a preferable strategy to control $V$. dahliae (van der Lek 1919) and in the early $20^{\text {th }}$ century several researchers and breeders reported resistance against $V$. dahliae in tomato. For example, in 1937 the development of tomato cultivar "Riverside" was reported that was resistant to both Fusarium and Verticillium wilts (Sherbakoff 1949). In 1932, a Peruvian wild tomato accession was identified which possessed relatively strong Verticillium resistance (Deseret News and Telegram 1955). In 1951, Schaible et al. reported that this Peruvian accession displayed qualitative resistance against Verticillium wilt which was based on a single dominantly inherited locus named "Ve" (Schaible et al. 1951). Although this Ve locus was rapidly introduced in commercial cultivars, breeders struggled to map the position of the Ve locus. First, evidence suggested that the Ve locus is located on chromosome 4 (Rick et al. 1959), which was later disproven by Kerr and Busch (1975). Later, a screening of a RIL population suggested that the Ve locus is located on chromosome 7, which was later contradicted by Diwan et al. (1999), who definitively demonstrated that $V e$ is located on chromosome 9. Further fine mapping and cloning showed that the Ve locus consists of two genes, encoding the RLPs Ve1 and Ve2 (Kawchuk et al. 2001), of which Ve1 was determined to be the causal resistance gene (Fradin et al. 2009). Although VeI resistance was welcomed by farmers and resulted in increased tomato yields (Deseret News and Telegram 1955), in 1957 the first resistance-overcoming strains of $V$. dahliae were reported that caused symptoms on Ve1 resistant plants (Alexander 1962). Consequently, the $V$. dahliae population was split up into race 1 and 2 , based on the ability to cause disease on Ver tomatoes, with race 1 strains 
being contained while race 2 strains are not. In 2012, de Jonge et al. (2012) reported that Ve1 recognises an effector named Ave1 which has an important virulence function for the pathogen. Race 2 strains lack Ave1 and are therefore generally less aggressive than race 1 strains. Although race 2 strains were initially only reported in the USA (Alexander 1962; Robinson 1957), they soon appeared also in Europe on multiple locations (Cirulli 1969; Tjamos 1976; Pegg and Dixon 1969). Currently, race 2 strains are present in most of the tomatoproducing regions of the world. Although global yield losses from $V$. dahliae race 2 strains are difficult to determine, yield losses from $10 \%$ to $30 \%$ have been reported in the USA (Bender and Shoemaker 1984; Grogan 1979). The increasing incidence of race 2 strains motivated researchers to search for novel resistance sources. Although multiple resistances in tomato were reported (Okie and Gardner 1982b, 1982a; Latterot 1984; Baergen et al. 1993; Stamova 2005), none of these studies reported to have identified QTLs or genes underlying these resistances. In 2017, Usami et al. reported qualitative resistance against $V$. dahliae based on a single dominantly inherited locus named " $V 2$ " (Usami et al. 2017). Although this V2 resistance provided resistance against multiple $V$. dahliae race 2 strains, several strains were identified which caused severe wilting symptoms on plants that carry V2 (Usami et al. 2017). Therefore, a third $V$. dahliae race was established to assign strains that escape $V 2$ recognition. According to the latest classification, $V$. dahliae strains which have overcome the resistance of Ve1 or V2 should be assigned to race 2 or 3 , respectively, whereas strains which have not overcome the resistance of Ve1 and V2 should be assigned to race 1 (Fig. 2). As V2 is not yet commercially exploited outside Japan, and no resistance sources have been reported yet that control race 3 strains of $V$. dahliae, additional resistance sources are desired.
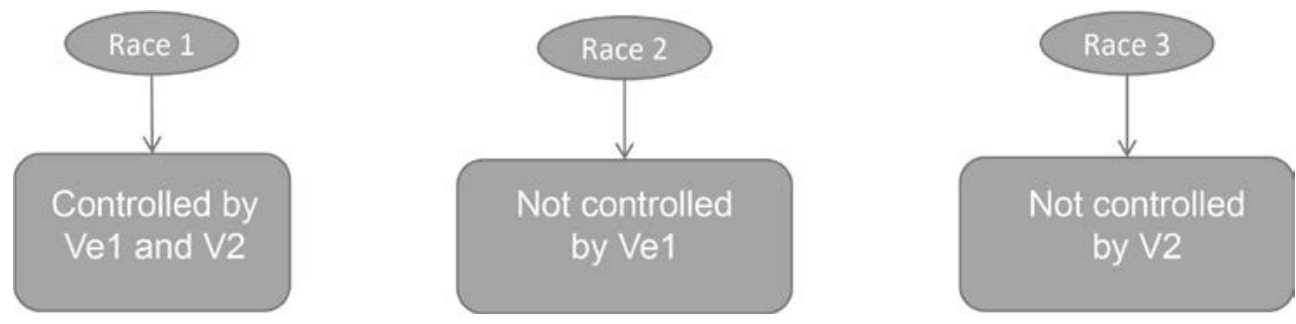

FICURE 2 | The latest classification of V. dahliae into race 1, 2 and 3, based on the resistance genes Ve1 (Fradin et al. 2009; Kawchuk et al. 2001) and the recently described V2 locus (Usami et al. 2017).

Previously, Yadeta (2012) screened a large collection of over 60 wild tomato accessions for resistance against $V$. dahliae race 2 . These screens revealed several accessions that displayed reduced Verticillium wilt symptoms to two race 2 strains of $V$. dahliae. Furthermore, to test whether these accessions are not tolerant, but resistant to these $V$. dahliae race 2 strains, fungal colonization in the stems was measured. Indeed, some accessions displayed reduced $V$. dahliae colonization, thereby confirming that these accessions are genuinely resistant to these $V$. dahliae strains. 


\section{Scope and outline of this thesis}

The main objective of this $\mathrm{PhD}$ thesis research was to verify several of the resistance sources that were previously identified by Yadeta (2012) against race $2 \mathrm{~V}$. dahliae, and to identify genes or QTLs underlying this resistance. During this $\mathrm{PhD}$ research, the V2 resistance locus was reported by Usami et al. (2016), giving rise to a re-classification of $V$. dahliae into 3 races (Fig. 2). Therefore, we also sought to identify the avirulence factor of $V$. dahliae race 2 strains that is recognized by $\mathrm{V} 2$.

To find a robust method to accommodate large-scale resistance screens with $V$. dahliae on tomato, we compared and assessed multiple phenotyping methods in chapter 2 . Furthermore, we aimed to optimize the inoculation and phenotyping procedures in order to increase the robustness and reproducibility of our disease screens. With the optimized phenotyping and inoculation method, we performed the $V$. dahliae screens in the remainder of the thesis.

In chapter 3, we compared the $V$. dahliae symptom expression on multiple wild tomato accessions. We identified three wild accessions, S. pimpinellifolium VG-3, S. pimpinellifolium VG-21 and S. cheesmanii VG-20, of which especially S. pimpinellifolium VG-21 and S. cheesmanii VG-20 displayed a broad-spectrum resistance against a diverse selection of $V$. dahliae strains and a $V$. nonalfalfa strain. By measuring the colonization of $V$. dahliae in the lower stems, we confirmed that especially $S$. pimpinellifolium VG-21 and S. cheesmanii VG-20 are resistant to most of the strains of which the colonization was assessed.

To unravel the genetics of the resistance of S. pimpinellifolium VG-3, S. pimpinellifolium VG-21 and S. cheesmanii VG-20, we developed RIL populations with the susceptible S. lycopersicum Moneymaker as the mother. In chapter 4, we describe the QTL mapping analysis for the resistance of S. pimpinellifolium VG-3 and S. pimpinellifolium VG-21. Unfortunately, we were unable to identify QTLs for the resistance of both accessions. However, surprisingly, in the population of S. pimpinellifolium VG-3, we identified a QTL on chromosome 3 contributed by Moneymaker that was associated with reduced $V$. dahliae-induced yellowing symptoms on the lower leaves.

In chapter 5, we describe the QTL mapping analysis for the V. dahliae resistance of $S$. cheesmanii VG-20. In this analysis, we identified three QTLs contributed by the $S$. cheesmanii VG-20 genome that were associated with reduced $V$. dahliae-induced stunting symptoms. Furthermore, we again identified a QTL on chromosome 3 contributed by Moneymaker, which overlapped with the QTL on chromosome 3 we mapped in the S. pimpinellifolium VG-3 RIL population. However, in this analysis, the QTL on chromosome 3 was associated with $V$. dahliae-induced stunting, whereas the QTL in the VG-3 population was associated with yellowing symptoms of the lower leaves. 
In chapter 6, we performed a comparative genomics analysis to identify the avirulence factor of race 2 strains that is recognized by V2 of the rootstock cultivar Aibou. Based on pathogenicity profiling, a collection of $V$. dahliae strains was categorized into race 2 and 3. Based on the availability of previously sequenced genomes and Oxford Nanopore Technology sequencing of additional genomes, we performed comparative genomics and compared the genomes of the race 2 and 3 strains. We identified race 2 -specific genomic sequence of $277 \mathrm{~kb}$ that contains two candidate effector genes that are both predicted to be secreted and are expressed in planta, of which future functional analysis will have to reveal which one encodes the genuine avirulence factor that is recognized by V2.

Finally, in chapter 7, I integrate and discuss the results presented in this $\mathrm{PhD}$ thesis, together with perspectives for future research on the resistance against $V$. dahliae in tomato. Furthermore, I discuss how the findings presented in this thesis may be used for breeding tomatoes with increased Verticillium wilt resistance. 



\title{
Chapter 2
}

\section{Evaluation of phenotyping and inoculation procedures for the identification of enhanced resistance against Verticillium dahliae in tomato}

\author{
Jasper P. Vermeulen ${ }^{1,2, \%}$, \\ Katharina Hanika ${ }^{1,2, *}$, \\ Bart P.H.J. Thomma ${ }^{1,3}$, \\ Yuling Bai², \\ Henk J.Schouten ${ }^{2}$
}

* These authors contributed equally to this work.

'Laboratory of Phytopathology, Wageningen University \& Research, 6708 PB Wageningen, The Netherlands. ${ }^{2}$ Laboratory of Plant Breeding, Wageningen University \& Research, 6708 PB Wageningen, The Netherlands.

${ }^{3}$ Cluster of Excellence on Plant Sciences (CEPLAS), University of Cologne, Botanical Institute, Cologne, Cermany. 


\section{Abstract}

Plants possess an innate immune system that provides resistance against most pathogens and pests. Still, particular pathogens evolved to overcome this resistance. In agriculture, a common approach to control such pathogens is to breed for genetic resistance. This approach aims at the exploration of plant germplasm for resistance sources, identification of the underlying resistance genes or quantitative trait loci (QTLs), followed by the introduction of these genes or QTLs into commercial cultivars. In order to reliably explore germplasm for resistance sources, an accurate method for phenotyping is essential. In other words, the phenotyping method should provide a good "discriminative power" to be able to detect differences in symptom expression between host genotypes. In the search for resistance against the vascular wilt pathogen Verticillium dahliae, various symptoms have been used to score disease development. The most commonly scored symptoms are reduced overall plant stature and size (stunting) and foliar symptoms such as yellowing, chlorosis and necrosis. In this study, we compared the discriminative power of several plant size-related disease symptoms on tomato in $V$. dahliae resistance screens. We obtained the highest discriminative power to detect differences in symptom expression between host genotypes by measuring the canopy area of $V$. dahliae-inoculated plants. The discriminative power was furthermore higher at 21 days after inoculation than at 14 days after inoculation. To assess whether the discriminative power of scoring the canopy area as a phenotyping method could be further optimized, we attempted to streamline the root-dipping inoculation method. We could not find a meaningful effect on the discriminative power by increasing the inoculum concentration, trimming of the roots prior to dipping them in inoculum or applying nutrients to the soil after the inoculation. 


\section{Introduction}

Plants are continuously exposed to a wide range of organisms, including a wide diversity of insects and microbes, some of which are potentially harmful. To protect themselves against pests and pathogens, plants possess a sophisticated immune system. Nevertheless, particular pests and pathogens can overcome these immune responses and cause disease. For example, the tomato is susceptible to over 200 different plant pests and pathogens (Jones 1991). Although the use of chemical control agents has been a common approach to tackle these organisms, the use of such chemicals has become increasingly restricted over the last decades (Lamichhane et al. 2016). Another strategy to combat pests and pathogens is to breed for resistance (Bai and Lindhout 2007, 2008). Like many other genetic traits, also resistance can be classified as either qualitative or quantitative (Corwin and Kliebenstein 2017). Qualitative resistance is genetically relatively simple and is based on single $R$ - or $S$-genes, whereas quantitative resistance is based on multiple, often small-effect loci. Because of its simple genetics, qualitative resistance has been studied and used in breeding more frequently than quantitative resistance (St.Clair 2010). As quantitative resistance is based on many loci that often make a limited contribution on their own, carefully designed disease screens and sensitive, accurate and robust phenotyping methods are crucial to successfully study the genetics of such resistance.

Vascular wilt pathogens typically concern soil-borne organisms that infect plants through the roots and invade the xylem vessels to spread to distal tissues of the plant host, often leading to wilting symptoms (Yadeta et al. 2013). Throughout the majority of the disease cycle, the pathogen is confined to the interior of the plant and pathogen colonization is often difficult to assess. An important vascular pathogen of tomato is Verticillium dahliae, an ascomycete fungus that belongs to the Verticillium genus. The Verticillium genus consists of ten species, of which five have a mostly saprophytic lifestyle, and the other five are plant pathogens (Inderbitzin et al. 2011). V. dahliae infects its hosts through the roots and then attempts to penetrate the root cortex to enter the xylem. Once in the xylem, it produces conidiospores that are carried with the sap stream and spread throughout the plant. Although symptoms depend largely on the host and environmental conditions, they may include stunting, vascular browning, wilting, yellowing and necrosis of the leaves (Fradin and Thomma 2006). Because V. dahliae resides inside the plant throughout most of the disease cycle, most fungicides are ineffective. However, resistance breeding is an alternative approach to control this disease.

Although several resistance sources to $V$. dahliae have been identified in tomato, only one resistance gene, Ve1, has been successfully mapped and cloned thus far (Kawchuk et al. 2001; Fradin et al. 2009). Soon after the deployment of this resistance gene, resistance-overcoming race 2 strains emerged (Alexander 1962; Robinson 1957). Therefore, identification of additional resistance genes or quantitative trait loci (QTLs) is desired to control V. dahliae 
race 2 strains. Although QTLs have been identified for $V$. dahliae resistance in other crops (Rygulla et al. 2008; Antanaviciute et al. 2015; Bolek et al. 2005; Wang et al. 2008; Zhao et al. 2014; Toppino and Barchi 2016), these studies often report quantitative resistance based on multiple loci, often with small effects. To detect such small-effect loci, accurate methods are required to quantify $V$. dahliae symptoms or the colonization in plants. In other words, these methods should provide a high "discriminative power" to be able to accurately detect differences in symptom expression or pathogen colonization between host genotypes.

Generally, research on resistance against $V$. dahliae is performed under controlled conditions, to be able to achieve robust inoculation protocols that result in reproducible disease phenotypes. One of the most commonly used $V$. dahliae inoculation methods for tomato is the root-dipping method, which involves dipping tomato roots directly into a $V$. dahliae conidiospore suspension (Papadaki et al. 2017; Shittu et al. 2009; Uribe et al. 2014; Parisi et al. 2016; Fradin et al. 2009). Alternative inoculation methods include the injection of conidiospores into the stem, watering of the soil with conidial suspensions, or the introduction of microsclerotia into the soil (Jiménez-Díaz et al. 2017; Bletsos et al. 2003; Buhtz et al. 2015; Antoniou et al. 2008; Depotter et al. 2019).

Because $V$. dahliae symptoms vary considerably among host species, methods to phenotype $V$. dahliae also differ between host species (Fradin and Thomma 2006). In tomato, the most common methods for phenotyping are scoring systems that categorize plants based on the severity of foliar symptoms such as wilting or yellowing (Chen et al. 2004; Busch and Smith 1981; Shittu et al. 2009; Jiménez-Díaz et al. 2017). For example, plants can be scored based on the number of leaves that are affected by yellowing and wilting on a scale from $0-5$, (Shittu et al. 2009). Other methods focus on quantitative determination of the impact of $V$. dahliae infection on elements of plant development, for example by measuring reduced plant height, stem length, fresh weight or canopy diameter, collectively referred to as stunting (Yadeta 2012; Papadaki et al. 2017). Finally, methods exist to quantify the colonization of $V$. dahliae using real-time PCR, or to qualitatively assess resistance by fungal outgrowth assays (Lievens et al. 2006; Fradin et al. 2009). Since solely the scoring of symptom expression cannot always distinguish tolerant from resistant plants, quantification of the colonization of $V$. dahliae needs to be performed to conclusively determine whether a host genotype is resistant to $V$. dahliae. However, these methods are generally more laborious because they require further processing of samples, in contrast to the direct measurements of symptoms. These methods are therefore too labour-intensive for large-scale resistance screens or QTL mapping experiments and thus we focus on plant growth parameters to phenotype Verticillium wilt in tomato.

Our long-term goal is to screen for novel sources of resistance against $V$. dahliae in tomato. However, to accommodate large-scale screens, we decided to first compare phenotyping methods in order to identify the most robust method to be performed in our plant growth 
facilities. Next, we optimized inoculation procedures to further optimize the robustness of our disease screens to, eventually, be able determine the most effective method to detect differences in disease symptoms between different host genotypes.

\section{Materials \& methods}

\section{Plant and pathogen material}

All tomato accessions (Table 1) were grown in potting soil (Potgrond 4, Horticoop, Katwijk, The Netherlands) in the greenhouse (Unifarm, Wageningen University \& Research, the Netherlands) at $25^{\circ} \mathrm{C} / 19^{\circ} \mathrm{C}$ (day/night) with $60 \%$ relative humidity and a minimal light intensity of $100 \mathrm{~W} / \mathrm{m}^{2}$. V. dahliae race 2 strain DVDS26 was grown on potato dextrose agar $(\mathrm{PDA})$ at room temperature in the dark.

TABLE1 | Tomato genotypes used in this study.

\begin{tabular}{cc}
\hline Species & Accession \\
\hline Solanum lycopersicum & Moneymaker \\
\hline Solanum lycopersicum & Moneymaker 35S:Ve1 \\
\hline Solanum pimpinellifolium & VG-3 \\
\hline Solanum cheesmanii & VG-20 \\
Solanum pimpinellifolium & VG-21 \\
\hline Solanum pimpinellifolium & VG-22 \\
\hline Solanum pimpinellifolium & VG-55 \\
Solanum pimpinellifolium & VG-63 \\
\hline Solanum lycopersicum Moneymaker x & \\
Solanum pimpinellifolium VG-3 & RIL660 (F6) \\
Solanum lycopersicum Moneymaker x & \\
Solanum pimpinellifolium VG-3 & RIL708 (F6) \\
\hline
\end{tabular}

\section{V. dahliae inoculation \& phenotyping}

To screen the tomato accessions, $V$. dahliae inoculations were carried out with the root dipping method as described by Fradin et al. (2009). Phenotyping was done at 14- and 21days post-inoculation (dpi) by measuring the stem diameter $(\mathrm{cm})$ just above the cotyledons with a digital calliper and by taking top- and side view pictures of the plants. Plant height (cm) was measured from the side-view pictures from the cotyledons upwards, and canopy area $\left(\mathrm{cm}^{2}\right)$ and canopy diameter $(\mathrm{cm})$ were measured from the top view pictures using ImageJ (Schneider et al. 2012). Stunting was calculated as follows:

$$
\text { Stunting }(\%)=\left(1-\frac{\text { canopy area of } V . \text { dahliae-inoculated plant of genotype } x}{\text { average canopy area of mock-treated plants of genotype } x}\right) \times 100
$$


For the comparison of different inoculation protocols, two inoculum concentrations ( $1 \mathrm{x}$ $10^{6}$ and $1 \times 10^{7}$ conidiospores $/ \mathrm{mL}$ ) were used and roots of half of the plants were trimmed to approximately $1 \mathrm{~cm}$. Also, at two and at three weeks after inoculation, a nutrient solution (Table S1) was applied twice per week to half of the plants.

\section{Estimating the discriminative power of the resistance test}

Ten tomato genotypes (Table 1) were inoculated with the race 2 strain V. dahliae DVDS26. At 14and 21 days post-inoculation, the stem diameter, plant height, canopy diameter, and canopy area were scored on mock-inoculated and $V$. dahliae-inoculated plants as described above. To estimate the discriminative power of the $V$. dahliae-associated symptoms, a one-way ANOVA was performed on these measurements. In this ANOVA, we tested for each symptom, per genotype, for significant differences between mock-inoculated and $V$. dahliae-inoculated plants. The experiment number was included in the analysis as a blocking factor. Where necessary, we performed a square-root or log10-transformation to guarantee the data met the normality and equality of variance assumptions. To estimate the discriminative power of each $V$. dahliae-associated symptom, the F-value from the ANOVA of the interaction between genotype * treatment was used. This F-value reflects the significance of the difference of the effect of the $V$. dahliae inoculation on plant size between genotypes.

\section{Results}

\section{Canopy area measurements at 3 weeks after inoculation provide the highest discriminative power to distinguish resistance level differences}

In order to explore tomato germplasm for sources of resistance against race $2 \mathrm{~V}$. dahliae strains, we queried for a phenotyping method to score Verticillium wilt disease that would provide the highest degree of resolution. In other words, this phenotyping method should provide the best discriminative power to best detect differences in $V$. dahliae symptoms between host genotypes. This discriminative power can be estimated with a one-way ANOVA, by testing which method yields the most significant differences in symptom expression between genotypes. Using a panel of ten tomato genotypes (Table 1), we compared the discriminative power of multiple phenotyping methods. Based on our earlier observations in greenhouse trials with $V$. dahliae-inoculated tomato plants, for instance the trials described by Fradin et al. (2009) and Yadeta (2012), we know that symptoms start to develop around $10 \mathrm{dpi}$, after which they aggravate. Taking the practicalities of large-scale screenings in mind, in which we prefer to terminate experiments 3 weeks after inoculation, we decided to focus the phenotyping efforts on 14 and 21 dpi. Initially, we aimed to assess both the scoring foliar $V$. dahliae symptoms and plant-size related Verticillium wilt symptoms. However, unfortunately, some of the tomato genotypes, especially $S$. 
cheesmanii VG-20, developed yellowing of the lower leaves in mock-inoculated plants (Fig S1). Simultaneously, several plants of the susceptible Moneymaker did not develop apparent yellowing symptoms upon inoculation with V. dahliae strain DVDS26. Therefore, we concluded that yellowing cannot be used as a symptom that is consistently associated with Verticillium wilt disease. Consequently, we discarded yellowing of leaves as a trait to be scored for $V$. dahliae susceptibility. Therefore, we continued with measurements of the reduction in stem diameter, canopy diameter, canopy area, and plant height on mockinoculated and $V$. dahliae-inoculated plants. Of these parameters, canopy area displayed the highest discriminative power both at 14 days and 21 days post-inoculation. Moreover, the discriminative power of the canopy area at 21 dpi was higher than at 14 dpi (Fig. 1).
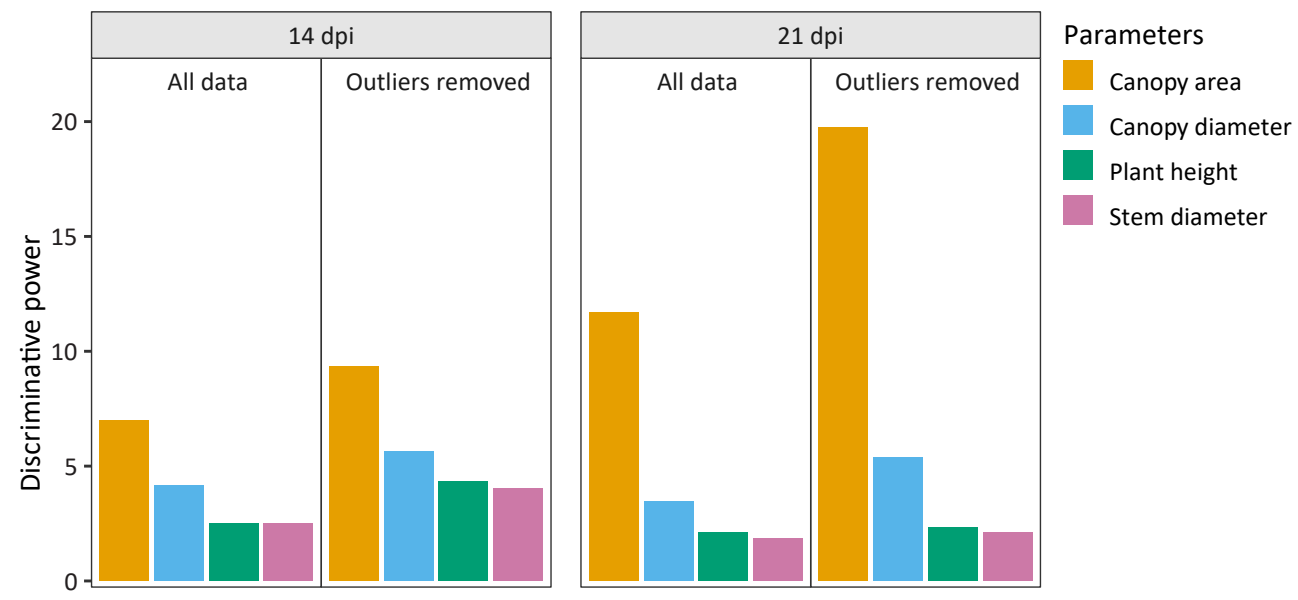

FIGURE 1 | Estimated discriminative power of canopy area, canopy diameter, plant height and stem diameter at 14 and 21 days after inoculation with V. dahliae DVDS26, based on the complete dataset and on the dataset from which outliers were or were not removed based on the studentized residuals (Table S3). Discriminative power was estimated with a one-way ANOVA, of which the F-value of the interaction genotype * treatment was used as the value for discriminative power.

Given the typically relatively large degree of variation in $V$. dahliae symptoms among plants of the same genotype, we also assessed the effect of the removal of outliers on the discriminative power of each of the symptoms. Removal of these outliers (Table S3) yielded a clear effect on the canopy area and canopy diameter, as it increased the discriminative power both at 14 and $21 \mathrm{dpi}$. At $14 \mathrm{dpi}$, outlier removal also resulted in a slight increase in the discriminative power of plant height and stem diameter. Overall, our analysis thus demonstrates that canopy diameter measurements at 21 dpi provide the highest discriminative power to distinguish resistant from susceptible genotypes, and that outlier removal may help to further improve the discriminative power of the resistance test. 


\section{Optimization of the inoculation procedure}

In order to assess whether the scoring of canopy area at $21 \mathrm{dpi}$ as a phenotyping measure could be optimized further, an attempt was made to optimize the inoculation method. To do this, two wild tomato accessions that were previously found by Yadeta (2012) to display a relatively high degree of resistance against $V$. dahliae race 2 were selected together with the susceptible control Moneymaker. The effect of three modifications of the rootdipping inoculation method on the discriminative power of the disease test was evaluated. First, the effect of the inoculum concentration was tested by increasing the conidiospore concentration from $10^{6}$ to $10^{7}$ conidiospores/ml (Fradin et al., 2009; Parisi et al., 2016; Jiménez-Díaz et al., 2017; Tsolakidou et al., 2019). Secondly, the addition of nutrients (Table Si) twice a week after the second week post sowing was assessed. Finally, trimming of the roots before inoculation was tested as this has been suggested to promote $V$. dahliae infection (Papadaki et al. 2017; Parisi et al. 2016).

In contrast to the different phenotyping methods, only minordifferences in the discriminative power of the different inoculation methods were detected (Fig. 2). No significant effect of the increase in conidiospore concentration on stunting of $V$. dahliae-inoculated plants was found for any of the tested genotypes, neither with or without root-trimming or nutrient application (Fig. 3). Furthermore, while the addition of nutrients significantly increased the canopy area of mock-treated plants of all genotypes (Fig. S2), we found that the addition of nutrients had no significant effect on $V$. dahliae-induced stunting for any of the genotypes (Fig. 3). Interestingly, the application of nutrients reduced the overall variation in stunting of V. dahliae-inoculated VG-20 plants and reduced the number of yellowing leaves of mockinoculated plants (Fig. S1). Finally, although no effect of root-trimming on the canopy area of mock-inoculated plants was detected (Fig. S2), also no effect of root-trimming on $V$. dahliae-induced stunting was observed for any of the genotypes (Fig. 3). Collectively, our data thus indicates that increasing the conidiospore concentration, root-trimming and nutrient application do not improve the discriminative power of the resistance test.

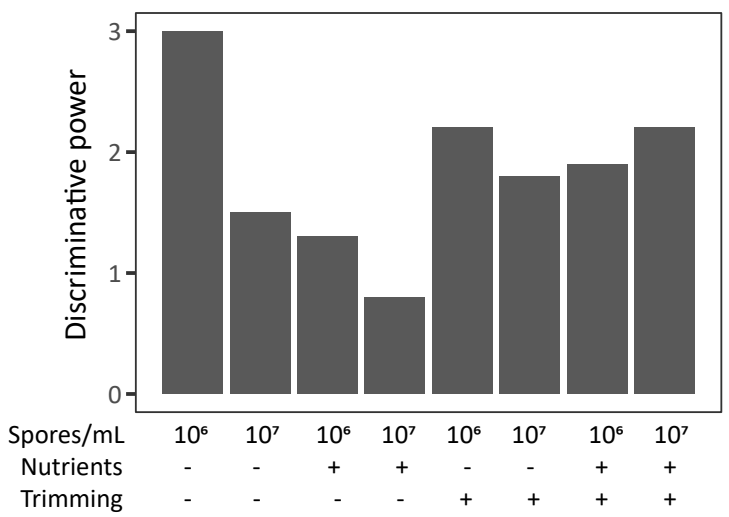

FICURE 2 | Estimated discriminative power of canopy area at 21 days after inoculation with V. dahliae DVDS26. Outliers were removed based on the studentized residuals (Table S4). Discriminative power was estimated with a one-way ANOVA, of which the F-value of the interaction between the genotype and the treatment. 


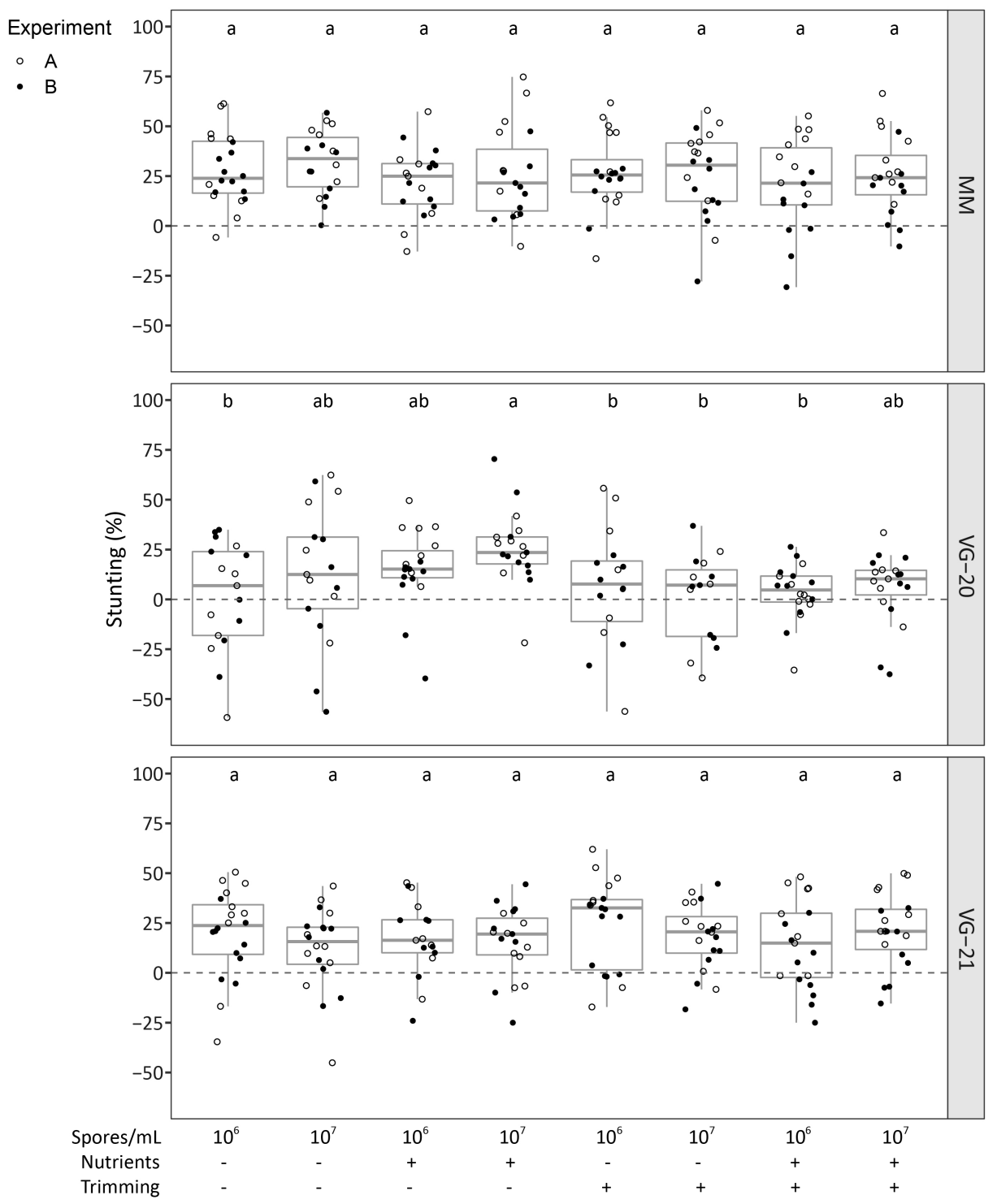

FIGURE 3 | Stunting (\%) based on the canopy area of V. dahliae-inoculated plants when compared with mockinoculated plants at 21 dpi of the tomato genotypes Moneymaker (top), VG-20 (middle) and VG-21 (bottom). Conidiospore concentration, addition of nutrients and trimming of the roots were compared. The depicted data comes from two independent experiments (filled versus non-filled dots) with $n \geq 9$ (ANOVA with Fisher's unprotected LSD, $\alpha=0.001$ ). 


\section{Discussion}

Since the emergence of $V$. dahliae race 2 strains on tomato, several additional resistance sources to Verticillium wilt were reported in tomato and other crops (Okie and Gardner 1982b, 1982a; Latterot 1984; Baergen et al. 1993; Stamova 2005; Klosterman et al. 2009; Usami et al. 2017; Yadeta 2012). Although these studies all had the similar goal to identify resistances to Verticillium wilt and to unravel the underlying genetics, the methods to inoculate plants and subsequently phenotype Verticillium wilt symptoms varied considerably among these studies.

Typically, disease screens aim to compare disease symptoms among different host genotypes or different treatments, to draw conclusions about the effect of the host genotype or treatment on the susceptibility/resistance against the disease. To best be able to draw such conclusions, the phenotyping method must have a high discriminative power to detect differences in symptom expression between host genotypes. In this chapter, we compared several methods to measure V. dahliae symptoms on tomato plants. By comparing the effect of $V$. dahliae-inoculation on the plant height, stem diameter, canopy area and canopy diameter, we demonstrated that some parameters better detect differences in symptom expression among tomato genotypes than other methods. We demonstrated that differences in symptom expression were most profound when the canopy area is measured at 21 dpi. The effect of $V$. dahliae -inoculation differed less profoundly between the tomato genotypes when stem diameter or plant height were determined.

The differences in the discriminative power of the parameters that were assessed in our study are not very surprising. First of all, the accuracy of some measurements may be better than that of other measurements. For example, we observed that the thickness of the stems of some wild tomato accessions was irregular, increasing variation in the stem diameter measurements and thereby likely reducing its discriminative power. Secondly, the difference in discriminative power of the canopy area and canopy diameter may be because canopy diameter solely measures the distance between the furthest leaf tips. Canopy area, in contrast, captures the area of the entire canopy and therefore also captures changes in leave size. Besides differences in the accuracy of the measurements, V. dahliae infection may also affect specific aspects of plant size to a more considerable extent than it affects other aspects. The higher discriminative power of canopy area and canopy diameter may thus also indicate that Verticillium infection has a stronger effect on canopy size than it has on plant height or stem diameter. In agreement with this, the susceptible control Moneymaker displayed a greater reduction in canopy area and canopy diameter than in plant height or stem diameter upon inoculation with $V$. dahliae (Table S2).

Overall, our analysis thus demonstrates that when searching novel resistance sources against $V$. dahliae, determining the right parameter to score disease symptoms is an important success factor for finding such resistance sources, including the subsequent QTL mapping 
analyses. Based on the appropriate parameter, the stunting of $V$. dahliae-inoculated plants should be calculated relative to mock-inoculated plants of the same genotype instead of directly comparing surface areas. In this way, genotypes which differ in size in absence of $V$. dahliae inoculation can still be compared with respect to their $V$. dahliae susceptibility.

Besides growth-related Verticillium wilt symptoms, many studies also score foliar symptoms such as wilting, yellowing and necrosis of the leaves. Thus, we initially aimed to also include these symptoms in our analysis. However, our Moneymaker plants did not develop apparent yellowing symptoms on all $V$. dahliae-inoculated plants, neither at $14 \mathrm{dpi}$ nor at 21 dpi. Simultaneously, mock-inoculated VG-20 plants also developed yellowing leaves in nutrient-deficient conditions (Fig. S1), indicating that yellowing of leaves is not always necessarily associated with $V$. dahliae infection. This thus indicates that yellowing symptoms could not be used to accurately identify genotypes which are more resistant than Moneymaker in our set of tomato germplasm.

Besides testing different phenotyping parameters to measure disease caused by $V$. dahliae, we also compared different inoculation methods to further optimize our assays. We assessed the effect of conidiospore concentration, nutrient application, and root-trimming on the discriminative power of the disease test. Although the highest discriminative power was found with $10^{6}$ conidiospores/ml without trimming of roots and nutrient applications (Fig. 2), the differences in discriminative power between inoculation methods were much less pronounced than the differences between the different phenotyping methods (Fig. 1). No clear differences in stunting were observed between the different inoculation treatments. Firstly, the two most commonly used conidiospore concentrations were tested for their effect on stunting (Fradin et al., 2009; Parisi et al., 2016; Jiménez-Díaz et al., 2017; Tsolakidou et al. , 2019). As no difference was found, it can be concluded that a concentration of $10^{6}$ conidiospores $/ \mathrm{ml}$ is sufficient to result in a robust $V$. dahliae infection. Possibly, the use of a higher conidiospore concentration does not increase stunting, as it does not lead to more penetration sites or higher levels of xylem colonization. Potentially, the use of a lower concentration may reduce the infection efficiency, but this would need to be tested further.

Some studies describe the application of nutrients during disease assays with $V$. dahliae (Shittu et al. 2009; Jiménez-Díaz et al. 2017; Parisi et al. 2016). However, a direct effect on disease development was not evaluated in those studies. In our study, the addition of nutrients did not affect overall stunting of the genotypes, although it mitigated yellowing in VG-20 and reduced the overall variation of this genotype (Fig. 3 and S1). This suggests that adding nutrients can affect symptom development for particular genotypes.

Finally, we found that root-trimming as described in previous studies (Papadaki et al. 2017; Parisi et al. 2016) does not aid $V$. dahliae infection, as no effect on stunting was found. It may be speculated that root-trimming creates additional wounds which can be used by $V$. dahliae as an entry point for infection. However, roots have many natural openings, such as sites 
of lateral root emergence, and furthermore they are unavoidably further damaged during uprooting before inoculation. Consequently, the potential benefit of root-trimming for $V$. dahliae infection may be limited. Furthermore, trimming drastically reduces the size of the roots and therefore reduces the available root surface to which conidiospores could attach. As no beneficial effect of root-trimming on stunting could be found, we demonstrate that it is not necessary to trim the roots before infection under our experimental conditions.

Collectively, our study demonstrated that canopy area at 21 days post-inoculation yields the best discriminative power to detect differences in $V$. dahliae-symptom expression in our set of tomato genotypes and under our experimental conditions. Furthermore, we demonstrated that applying nutrients to the soil post-inoculation, trimming the roots of seedlings prior to inoculation or increasing the inoculum concentration from $10^{6}$ to $10^{7}$ conidiospores $/ \mathrm{ml}$ has no meaningful beneficial effect on the discriminative power of the resistance test. 


\section{Supplemental material}

SUPPLEMENTARY TABLE 1 | Composition of nutrient solution used in this study.

\begin{tabular}{llll}
\hline Macro-elements & $\mathrm{mmol} / \mathrm{L}$ & Micro-elements & $\mu \mathrm{mol} / \mathrm{L}$ \\
\hline $\mathrm{NH}$ & 1.2 & $\mathrm{Fe}$ & 35.0 \\
\hdashline $\mathrm{K}$ & 7.2 & $\mathrm{Mn}$ & 8.0 \\
\hline $\mathrm{Ca}$ & 4.0 & $\mathrm{Zn}$ & 5.0 \\
$\mathrm{Mg}$ & 1.82 & $\mathrm{~B}$ & 20.0 \\
$\mathrm{NO}$ & 12.4 & $\mathrm{Cu}$ & 0.5 \\
\hline $\mathrm{SO}_{4}$ & 3.32 & $\mathrm{Mo}$ & 0.5 \\
$\mathrm{P}$ & 1.0 & & \\
\hline
\end{tabular}

SUPPLEMENTAL TABLE 2 | Plant size parameters of the susceptible controls.

\begin{tabular}{|c|c|c|}
\hline \multirow{2}{*}{ Treatment group } & \multicolumn{2}{|c|}{ Canopy area $\left(\mathrm{cm}^{2}\right)$} \\
\hline & Moneymaker & Moneymaker 35S:Ve1 \\
\hline V. dahliae-inoculated plants & $301.89(102.6)$ & $266.66(80.96)$ \\
\hline Mock - inoculated plants & $527.38(88.99)$ & $470.68(114.59)$ \\
\hline Stunting of V. dahliae inoculated plants & $42.76(19.4)$ & $43.35(17.2)$ \\
\hline \multirow[t]{2}{*}{ Treatment group } & \multicolumn{2}{|c|}{ Canopy diameter (cm) } \\
\hline & Moneymaker & Moneymaker 35S:Ve1 \\
\hline V. dahliae-inoculated plants & $30.37(5.96)$ & $28.27(5.23)$ \\
\hline Mock - inoculated plants & $42.43(5.07)$ & $39.24(3.61)$ \\
\hline Stunting of $V$. dahliae inoculated plants & $28.41(14.1)$ & $27.97(13.32)$ \\
\hline \multirow[t]{2}{*}{ Treatment group } & \multicolumn{2}{|c|}{ Height $(\mathrm{cm})$} \\
\hline & Moneymaker & Moneymaker 35S:Ve1 \\
\hline V. dahliae-inoculated plants & $27.52(4.74)$ & $21.89(3.80)$ \\
\hline Mock - inoculated plants & $31.69(2.80)$ & $24.95(3.80)$ \\
\hline Stunting of V. dahliae inoculated plants & $13.17(14.95)$ & $12.28(15.22)$ \\
\hline
\end{tabular}

Averages of canopy area, canopy diameter and height of mock-inoculated and V. dahliae-inoculated plants of the susceptible control Moneymaker and race 1 resistant control Moneymaker 35S:Ve1. Plants were inoculated with the $V$. dahliae race 2 strain DVDS26. Stunting represents the reduction in size of $V$. dahliae-inoculated plants relative to the average size of the mock-inoculated plants of the same genotype. Numbers in brackets indicate the standard deviation. 
SUPPLEMENTAL TABLE 3 | Number of removed outliers in the analysis of the data presented in figure 1

\begin{tabular}{|c|c|c|c|c|}
\hline \multirow{2}{*}{ Genotype } & \multicolumn{4}{|c|}{14 days post inoculation } \\
\hline & Canopy area & Stem diameter & Plant Height & Canopy diameter \\
\hline Moneymaker & 1 & 0 & 0 & 0 \\
\hline MM_Ve1 & 0 & 2 & 0 & 0 \\
\hline VG-3 & 0 & 1 & 0 & 0 \\
\hline VG-20 & 2 & 1 & 0 & 0 \\
\hline VG-21 & $\circ$ & 2 & 0 & $\circ$ \\
\hline VG-22 & 0 & 1 & 1 & 2 \\
\hline VG-55 & 3 & 0 & 0 & 3 \\
\hline VG-63 & 0 & 0 & 2 & $\circ$ \\
\hline RIL660 & $\circ$ & 2 & 1 & 0 \\
\hline RIL708 & 0 & 0 & 0 & 0 \\
\hline Total & 6 & 9 & 4 & 5 \\
\hline \multirow{2}{*}{ Genotype } & \multicolumn{4}{|c|}{21 days post inoculation } \\
\hline & Canopy area & Stem diameter & Plant Height & Canopy diameter \\
\hline Moneymaker & 0 & 2 & 0 & 2 \\
\hline MM_Ve1 & $\circ$ & 0 & 1 & $\circ$ \\
\hline$V G-3$ & 2 & 1 & 0 & 0 \\
\hline VG-20 & 1 & 0 & 2 & $\circ$ \\
\hline VG-21 & 1 & 0 & 0 & 0 \\
\hline VG-22 & 0 & 0 & 1 & 1 \\
\hline VG-55 & 2 & 1 & 0 & 1 \\
\hline VG-63 & 2 & 1 & 0 & 2 \\
\hline RIL660 & 0 & 2 & 2 & 2 \\
\hline RIL708 & 0 & 0 & 0 & 2 \\
\hline Total & 8 & 7 & 6 & 10 \\
\hline
\end{tabular}


SUPPLEMENTAL TABLE 4 | Number of removed outliers in the analysis of the data presented in figure 2

\begin{tabular}{|c|c|c|c|c|c|c|c|c|}
\hline Trimming & - & - & - & - & + & + & + & + \\
\hline Nutrients & - & - & + & + & - & - & + & + \\
\hline Inoculum concentration & $10^{6}$ & $10^{7}$ & $10^{6}$ & $10^{7}$ & $10^{6}$ & $10^{7}$ & $10^{6}$ & $10^{7}$ \\
\hline Moneymaker & o & $\circ$ & 0 & 0 & 0 & 0 & 0 & 0 \\
\hline VG-20 & 3 & 2 & 0 & 0 & 3 & 3 & 0 & 0 \\
\hline VG-21 & 0 & ० & 2 & 1 & ० & 0 & 1 & 1 \\
\hline Total & 3 & 2 & 2 & 1 & 3 & 3 & 1 & 1 \\
\hline
\end{tabular}

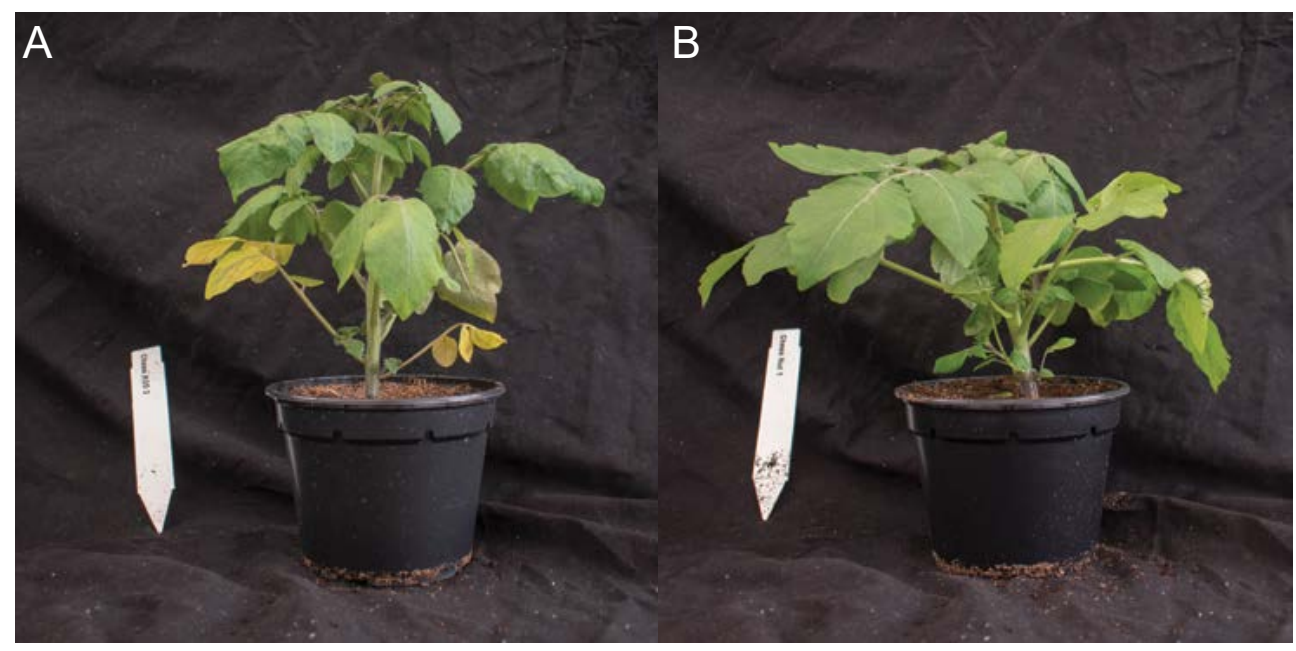

SUPPLEMENTAL FIGURE 1 | Appearance of non-inoculated plants of S. cheesmanii VG-20 at four weeks after sowing without nutrient addition (A) or after the receipt of additional nutrients twice a week after the $2^{\text {nd }}$ week post sowing (B).
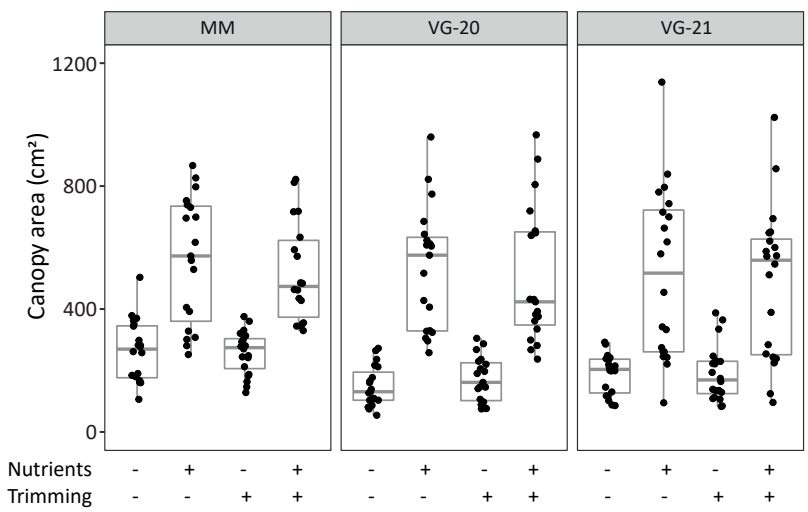

SUPPLEMENTAL FICURE 2 | Canopy area $\left(\mathrm{cm}^{2}\right)$ of mock-treated plants (21 dpi) with and without the addition of nutrients and with and without trimming of the roots. Different letter labels indicate significant differences as determined with a one-way ANOVA followed by a fishers LSD test $(\mathrm{p}<0.001)$. 



\title{
Chapter 3
}

\section{Screening of wild tomato germplasm reveals broad- spectrum resistance against Verticillium wilt disease in at least two accessions}

\author{
Jasper P. Vermeulen ${ }^{1,2}$, \\ Katharina Hanika ${ }^{1,2}$, \\ Danny Schipper², \\ Yuling $\mathrm{Bai}^{2}$, \\ Henk J.Schouten ${ }^{2}$, \\ Bart P.H.J. Thomma ${ }^{1,3}$
}




\section{Abstract}

Currently, Verticillium dahliae consists of three races, of which race 1 strains are contained by the resistance governed by VeI and V2, whereas race 2 and 3 have overcome the resistance governed $V e 1$ and $V 2$, respectively. Thus far, only $V e 1$ is widely exploited in tomato cultivation as the gene has been introduced in most tomato cultivars, whereas V2 has not yet been exploited commercially outside Japan. Since no $R$ genes are currently described which control race 3 strains of $V$. dahliae and $V 2$ is not widely available, additional resistance sources are required to control $V$. dahliae. Previously, a diverse collection of wild tomato accessions was screened for resistance against $V$. dahliae race 2 , revealing multiple tomato accessions which displayed reduced Verticillium wilt symptoms. Interestingly, these accessions did not display reduced Verticillium wilt symptoms when challenged with two $V$. dahliae race 1 strains. In this study, we confirmed the $V$. dahliae resistance of several of these wild tomato accessions. Solanum cheesmanii VG-20, and Solanum pimpinellifolium VG-3 and VG-21 displayed resistance against a diverse selection of $V$. dahliae strains and one $V$. nonalfalfa strain. In contrast to earlier findings, our analysis revealed that these accessions were also partially resistant to various $V$. dahliae race 1 strains. Furthermore, VG-20 and VG-21 also displayed resistance against a race 3 strain of $V$. dahliae, indicating that the resistance may be effective against strains of all currently known races of $V$. dahliae. Given that the resistances of VG-3, VG-20, and VG-21 appear to be of a broad-spectrum nature, they display more of the typical characteristics of quantitative resistance than of qualitative $R$-gene resistance. To confirm this hypothesis, further studies should be performed to unravel the genetic structure of the resistances of these accessions. 


\section{Introduction}

Microbial disease is ubiquitous in nature. It has been hypothesized that parasites constitute over half of the organisms on Earth (Windsor 1998), causing damage to a wide of variety of hosts. Plants are no exception, as plants are exposed to a wide range of parasites, including pathogens. To limit the damage caused by pathogens, plants employ two main defence strategies: resistance and tolerance. Resistance refers to mechanisms that prevent or limit pathogen infection or proliferation, while tolerance refers to mechanisms which aim to limit the amount of damage caused by the infection without controlling the pathogen colonization (Pagán and García-Arenal 2018; Roy and Kirchner 2000; Råberg 2014).

Plant resistance can be distinguished into quantitative and qualitative resistance. Qualitative resistance is in most cases based on single $R$ - or $S$-genes and segregates into discrete classes of resistant and susceptible phenotypes. $R$ genes commonly encode cytoplasmic immune receptors such as nucleotide binding-leucine rich repeat receptors (NLRs) or cell surface receptors such as receptor-like-proteins (RLPs) and receptor like kinases (RLKs) (St.Clair 2010; Corwin and Kliebenstein 2017), which recognise avirulence factors of the pathogens. $R$ genes often confer a race-specific, complete form of resistance that results in absence of disease and has therefore been commonly used in plant breeding. A large number of $R$ genes has been identified, characterized, and used in breeding programmes (Pandolfi et al. 2016). However, the high level of resistance provided by many $R$ genes can confer a strong diversifying selection on pathogen populations to select for pathogen variants that are no longer recognised by the $R$ gene, or that are able to suppress the resistance response in the plant. The strength of this diversifying selection depends largely on the fitness cost of losing the avirulence factor which is detected by the $R$ gene (Brown 2015). Still, $R$ gene-based resistance is not often very durable, which causes breeders to continuously need to seek for additional resistances (St.Clair 2010).

Quantitative resistance segregates into a more continuous distribution from resistant to susceptible phenotypes and is in most cases based on multiple, often small-effect loci (Corwin and Kliebenstein 2017). Because of its more complex biology and genetics, quantitative resistance against plant pathogens is still relatively poorly understood. Over the last decades, quantitative resistance has received increasing attention from breeders and phytopathologists. Because of its polygenic nature, quantitative resistance is thought to be generally more durable (St.Clair 2010; Corwin and Kliebenstein 2017). After all, since quantitative resistance is based on multiple, often small-effect genes, pathogen variants that overcome a partial quantitative resistance gene may have a smaller evolutionary advantage than pathogen variants that overcome a qualitative $R$ gene. However, due to the biological and genetic complexity of quantitative resistance, it has not been gained as much attention as qualitative resistance. 
When compared to resistance, tolerance to plant pathogens has received little attention from breeders and phytopathologists. Several examples of tolerance to plant pathogens have been reported (Roberts 1984; Politowski 1978; Mikaberidze and McDonald 2020), but the underlying genes and mechanisms are still mostly unknown. Because tolerance and resistance can produce similar phenotypes - a reduction in disease symptoms the two phenomena can easily be confused as they can only be discriminated based on quantification of pathogen colonization. For example, it has been demonstrated for potato that resistance against the fungal vascular pathogen Verticillium dahliae cannot always be distinguished from tolerance (Dan et al. 2001), which may cause breeders to select tolerant potato genotypes instead of resistant genotypes. Although both tolerance and resistance can result in reduced symptoms, only resistance thus reduces or prevents the accumulation of pathogen biomass in the plants and the field.

The genus Verticillium consists of ten species, of which five mainly have a saprophytic lifestyle and the other five are pathogenic (Inderbitzin et al. 2011). Of the five pathogenic Verticillium species, $V$. dahliae is especially problematic due to its wide host range and because it produces resting structures called microsclerotia which can remain viable in the soil for many years (Wilhelm 1955). Because the fungus remains inside the plant throughout most of its life cycle, most fungicides are ineffective. Employing genetic host resistance has been a popular approach to control this disease. The first resistance locus, Ve, was identified in 1953, which is now known to contain two RLP-encoding genes, Ve1 and Ve2, of which VeI is the functional R gene (Fradin et al. 2009; Kawchuk et al. 2001). Soon after the introduction of $V e 1$ into commercial cultivars, race 2 strains emerged which overcame Ve1-resistance (Alexander 1962). Recently, the tomato rootstock cultivar Aibou was reported to provide resistance against $V$. dahliae, which is likely based on a dominant locus called V2 (Usami et al. 2017). Interestingly, several V. dahliae strains were not contained on Aibou, suggesting that these strains have overcome the resistance governed by V2 (Usami et al. 2017). Therefore, $V$. dahliae strains which have overcome the resistance of Ve1 or V2 should be assigned to race 2 or 3 , respectively, whereas strains which have not broken the resistance of $V e 1$ and $V 2$ should be assigned to race 1.

Besides the resistance of the tomato rootstock Aibou, multiple other resistance sources against race 2 of $V$. dahliae were identified by Yadeta (2012). In this study, several wild tomato accessions displayed reduced stunting symptoms when challenged with two $V$. dahliae strains. Some tomato genotypes also displayed reduced $V$. dahliae colonization in the stems, confirming that these accessions are not tolerant, but resistant to these $V$. dahliae strains. Interestingly, the resistance of these accessions did not seem effective against two race 1 strains, as these accessions displayed clear Verticillium wilt symptoms when challenged with these strains (Yadeta 2012). In this chapter, we further assessed the resistance levels and spectrum of several wild tomato accessions described by Yadeta et al. (2012). We identified three wild tomato accessions, S. cheesmanii VG-20, S. pimpinellifolium 
VG-21 and S. pimpinellifolium VG-3, which displayed broad-spectrum resistance against a diverse set of Verticillium strains including, besides race 2 strains, also race 1 strains and a race 3 strain. Finally, all three accessions displayed reduced $V$. dahliae symptom expression when challenged with a race $2 \mathrm{~V}$. nonalfalfa strain.

\section{Materials \& methods}

\section{Preparation of inoculum and inoculation procedures}

Seedlings of Solanum lycopersicum Moneymaker, Solanum pimpinellifolium VG-21, VG-3, and Solanum cheesmanii VG-20 were inoculated with 15 different Verticillium strains. Of these strains, one belonged to the species $V$. nonalfalfa, whereas the other 14 belonged to $V$. dahliae (Table 1). For production of the inoculum for the inoculation, two petri dishes with potato dextrose agar (PDA) were inoculated per Verticillium strain from $-80^{\circ} \mathrm{C}$ glycerol stocks. The fungus was grown on plates at $24^{\circ} \mathrm{C}$ for at least five days. Using these cultures, three fresh petri dishes with PDA were inoculated per Verticillium strain and kept at $24^{\circ} \mathrm{C}$ for five to seven days. Then, conidiospores were harvested from the potato dextrose agar, washed, and resuspended in tap water to a final concentration of 106 conidiospores per $\mathrm{mL}$. For inoculation, ten-day-old seedlings were carefully uprooted and roots were washed in tap water. On average, of each tomato accession, 20 plants were used of which half were rootdipped for 10 minutes in water and the other half were root-dipped for 10 minutes in the inoculum. After inoculation, seedlings were placed in $14 \mathrm{~cm}$ pots and randomly distributed across the greenhouse compartment. Plants were grown in the greenhouse (Unifarm, Wageningen University \& Research, the Netherlands) at maximum temperatures of $26^{\circ} \mathrm{C} / 19^{\circ} \mathrm{C}$ (day/night) with $60 \%$ relative humidity and a minimum light intensity at daytime of $100 \mathrm{~W} / \mathrm{m} 2$. Day length was set at 16 hours, night length at 8 hours.

\section{Phenotyping of the stunting symptoms and statistical analysis}

Plants were phenotyped at 21 days post-inoculation (dpi). The canopy of individual plants was photographed from the top using a Nikon D3200 DSLR camera that was fixed on a tripod. Using ImageJ version 1.51f (Schneider et al. 2012), the canopy area of the plants was determined. The degree of stunting of Verticillium-inoculated plants was then calculated by comparing the size of the inoculated plant with the average size of all mock-treated plants of the same accession, using the following formula:

$$
\text { Stunting }(\%)=\left(1-\frac{\text { Canopy area of inoculated plant }}{\text { Average canopy area of all mock }- \text { treated plants of genotype } x}\right) * 100
$$

To test for significant differences in stunting, an ANOVA was performed. Outliers were detected based on the studentized residuals from the ANOVA analysis. Datapoints with studentized residuals below -2.5 or above 2.5 were considered as outliers and removed. 


\section{Determination of Verticillium biomass in infected plants}

To determine the colonization of $V$. dahliae in inoculated plants, a stem fragment of $2-3 \mathrm{~cm}$ was harvested from the stem directly above the cotyledons at 22-23 dpi. Stem fragments were placed in $15 \mathrm{~cm}$ long plastic tubes, which were stored in liquid nitrogen directly after the harvest. The stem fragments were then freeze-dried for at least 24 hours at a temperature below $-40^{\circ} \mathrm{C}$ and atmospheric pressure below 0.1 mbar. After freeze-drying, stems were manually ground to a powder with a metal rod. Next, DNA was isolated from the stems with a modified version of the procedure from Pak et al. (1997) (Appendix). To determine the Verticillium biomass, real-time PCR was performed on a CFX96 Touch Real-Time PCR Detection System (Bio-Rad, Veenendaal, The Netherlands) with the SsoAdvanced Universal SYBR Green Supermix (Bio-Rad, Veenendaal, The Netherlands). The real-time PCR programme consisted of an initial denaturation step of 10 minutes at $98^{\circ} \mathrm{C}$ followed by 41 cycles of $98^{\circ} \mathrm{C}$ for $15 \mathrm{~s}$ and $60^{\circ} \mathrm{C}$ for $1 \mathrm{~min}$. After the 41 cycles, a stepwise melting curve analysis was performed from 65 to $95^{\circ} \mathrm{C}$ with steps of $0.5^{\circ} \mathrm{C}$ and 5 seconds per step. The fungus-specific primer ITSI-F, which is based on the internal transcribed spacer region of the ribosomal DNA from Lievens et al. (2006), was used in combination with $V$. dahliae-specific reverse primer ST-Ve1-R to determine the amount of $V$. dahliae DNA in the DNA samples. To determine the amount of tomato DNA, primers designed on the tomato Elongation Factor $1 \alpha$ (Appiano 2016) were used. Of each stem sample, two technical replicates were tested with the real-time PCR. Stem samples of which the two technical replicates displayed a difference greater than $1 \mathrm{Ct}$ for the tomato amplicons were not further analysed. Relative levels of fungal biomass compared to the amount of host DNA were then determined with the 2- $\Delta \Delta \mathrm{Ct}$ method (Livak and Schmittgen 2001). Statistical differences were determined based on the $\Delta \Delta$ Ct values with a one-way ANOVA, followed by a Fishers' LSD test. Datapoints with studentized residuals below -2.5 or above 2.5 were considered as outliers and removed.

\section{Results}

\section{Four wild tomato accessions displayed reduced stunting upon inoculation with two} V. dahliae race 2 strains

To confirm and further study the previously described resistances (Yadeta 2012) we selected six wild tomato accessions (Table 2 , materials \& methods) which previously displayed a reduction in $V$. dahliae symptoms, and challenged these accessions with the race $2 V$. dahliae strains DVD3 and DVDS26. At 21 days post inoculation, we measured the stunting of $V$. dahliae-inoculated plants. Compared to the susceptible control Moneymaker, all six wild tomato accessions displayed reduced stunting symptoms when challenged with either $V$. dahliae DVD3 or DVDS26 (Fig. 1). All wild tomato accessions, except for accession VG-22, 
displayed a reduction in stunting symptoms when challenged with $V$. dahliae DVDS26. When challenged with V. dahliae DVD3, only VG-20, VG-21 and VG-63 displayed reduced stunting when compared with Moneymaker. Of the six accessions, only VG-20, VG-21 and VG-63 displayed reduced symptom expression to both race $2 \mathrm{~V}$. dahliae strains.

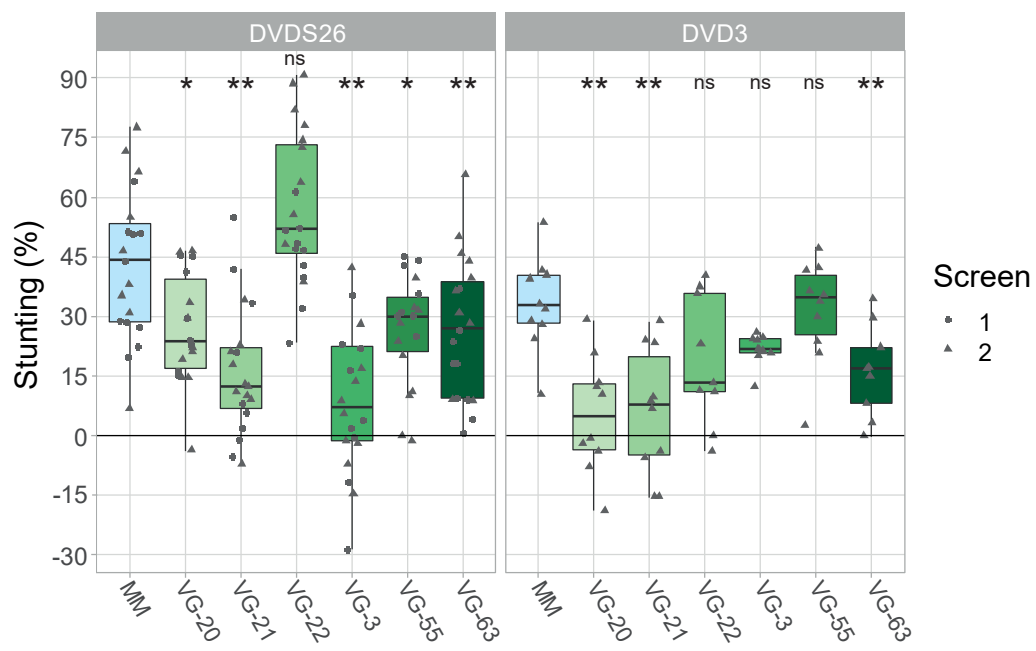

FICURE1|Stunting of wild tomato accessions challenged with V. dahliae race 2 strains DVD 3 and DVDS26 at 21 days post inoculation. "MM" represents the susceptible control S. lycopersicum Moneymaker. VG-2O is of the species S. cheesmanii, the remaining VG-numbers refer to wild accessions of the species $S$. pimpinellifolium. Asterisks indicate significant differences in stunting compared to Moneymaker; ${ }^{*} \mathrm{p}<0.01,{ }^{* * *} \mathrm{p}<0.005$. Significant differences were determined with a one-way ANOVA followed by a fishers LSD test.

\section{Wild tomato accessions VG-3, VG-21 and VG-20 displayed reduced stunting upon inoculation with a phylogenetically diverse set of Verticillium strains}

VG-20 and VG-21 were selected for further study, as these accessions displayed reduced stunting (Fig. 1) and reduced $V$. dahliae outgrowth in a previous study when challenged with both DVDS26 and DVD3 (Yadeta 2012). In addition, because for VG-3 a fully genotyped RIL population was available at Wageningen University, this accession was also included for further analysis. To further assess the resistance spectrum of VG-20 and of VG-21, a diverse set of $V$. dahliae strains was selected. To be able to make a phylogenetically diverse selection of strains, we mostly selected strains of which the genome sequence was available and for which a phylogenetic tree has been built. Where possible, at least one tomato-infecting race 2 strain was selected from each major branch of the phylogenetic tree (Fig. 2). As the none of the strains in the lowest major branch of the tree are pathogenic on tomato, no strains were selected from this branch. An additional $V$. dahliae strain from Australia and the Netherlands, and one V. nonalfalfa strain from Belgium was also included (Table 1). 
TABLE 1 | Overview of all Verticillium strains used in this study.

\begin{tabular}{|c|c|c|c|c|c|}
\hline Species & Strain & Race & Country of origin & Originally isolated from & $\begin{array}{c}\text { Genome } \\
\text { sequenced? }\end{array}$ \\
\hline V. dahliae & $\mathrm{JR2}$ & 1 & Canada & Solanum lycopersicum & Yes \\
\hline V. dahliae & $2009-650$ & 1 & Ukraine & Capsicum annuum & Yes \\
\hline V. dahliae & CBS38166 & 1 & Canada & Solanum lycopersicum & Yes \\
\hline V. dahliae & v52 & 1 & Austria & Capsicum annuum & Yes \\
\hline V. dahliae & St.1401 & 1 & USA & Pistacia vera & Yes \\
\hline V. dahliae & $\mathrm{CBS} 321.91$ & 2 & Netherlands & Solanum lycopersicum cv Criterium & No \\
\hline V. dahliae & DVDS29 & 2 & Canada & Soil & Yes \\
\hline V. dahliae & VdLs17 & 2 & California & Lactuca sativa & Yes \\
\hline V. dahliae & VPRI 42079 & 2 & Australia & Solanum lycopersicum & No \\
\hline V. dahliae & DVD161 & 2 & Canada & Solanum tuberosum & Yes \\
\hline V. dahliae & DVDS26 & 2 & Canada & Soil & Yes \\
\hline V. dahliae & $\mathrm{DVD}_{3}$ & 2 & Canada & Solanum tuberosum & Yes \\
\hline V. nonalfalfa & CBS451.88 & 2 & Belgium & Unknown & No \\
\hline V. dahliae & Gf1207 & 2 & Japan & Solanum lycopersicum 'Momotaro Sunny' & Yes \\
\hline V. dahliae & HOMCF & 3 & Japan & Solanum lycopersicum 'Momotaro CF' & Yes \\
\hline
\end{tabular}

TABLE 2 | Overview of all tomato accessions used in this study.

\begin{tabular}{cc}
\hline Species & Accession \\
\hline Solanum lycopersicum & Moneymaker \\
\hline Solanum pimpinellifolium & VG-3 \\
\hline Solanum cheesmanii & VG-20 \\
\hline Solanum pimpinellifolium & VG-21 \\
\hline Solanum pimpinellifolium & VG-22 \\
\hline Solanum pimpinellifolium & VG-55 \\
\hline Solanum pimpinellifolium & VG-63 \\
\hline
\end{tabular}




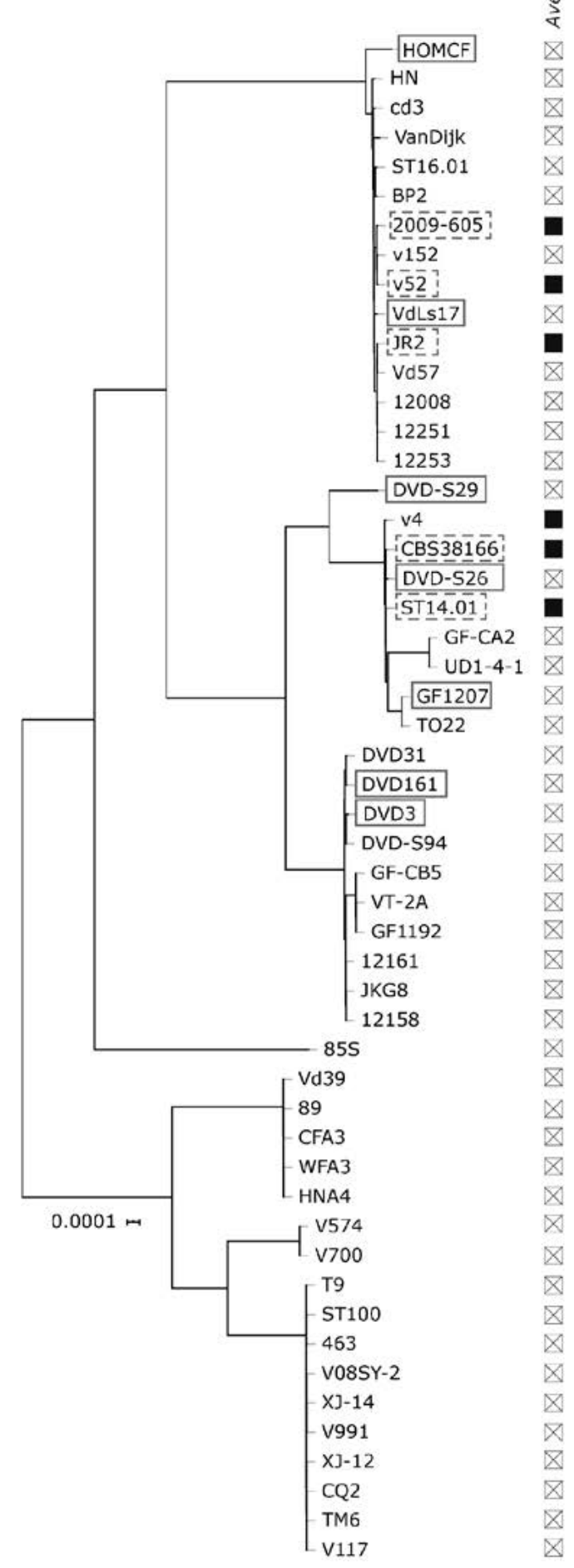

FICURE 2 | Phylogenetic tree of a selection of sequenced $V$. dahliae strains. Boxes with solid lines represent the race 2 or $3 \mathrm{~V}$. dahliae strains used in this study. Boxes with dashed lines represent the selected race $1 V$. dahliae strains. Phylogenetic relationships between sequenced V. dahliae strains were inferred using Realphy (Langmead and Salzberg 2012) Phylogenetic tree was modified from Chavarro-Carrero, (unpublished). 
Compared to Moneymaker, all three wild accessions displayed reduced stunting symptoms when challenged with all of the $V$. dahliae race 2 strains (Fig. 3A). Similarly, when challenged with the $V$. nonalfalfa race 2 strain CBS451.88, the accessions displayed a reduction in symptoms. Previously, Yadeta (2012) reported that these accessions displayed a higher level of stunting when inoculated with the race 1 strain JR2. However, VG-20, VG-21 and VG-3 still displayed reduced stunting when compared with Moneymaker plants, suggesting that these accessions may be at least partially resistant or tolerant to this strain. To further test the response of the three wild accessions to $V$. dahliae race 1 strains, we selected four $V$. dahliae race 1 strains from different branches of the phylogenetic tree (Fig. 2). Compared with Moneymaker, the three wild accessions displayed reduced stunting when challenged with these four race 1 strains, except for VG-20, which displayed no reduced stunting when challenged with $V$. dahliae strain v52. Finally, we challenged the wild accessions with two race 2 and two race 3 strains of $V$. dahliae from Japan (Usami et al. 2017). Of the three accessions, VG-20 and VG-21 displayed reduced stunting when challenged with both the race 2 strain GF1207 and the race 3 strain HOMCF, demonstrating that the resistance of VG-20 and VG21 is also effective against race 3 of $V$. dahliae. Overall, our analysis demonstrates that, when compared with Moneymaker, the wild accessions VG-3, VG-20 and VG-21 display reduced susceptibility towards a wide variety of Verticillium strains.

\section{VG-20 and VG-21 reduced the colonization of a diverse set of $V$. dahliae race 1,2 and 3 strains}

To verify that VG-3, VG-21 and VG-20 restrict the colonization of V. dahliae or whether they solely reduce the damage from the $V$. dahliae infection, the biomass of $V$. dahliae in the lower stems of $V$. dahliae-inoculated plants was measured and compared to the $V$. dahliae biomass in inoculated Moneymaker plants. Analogous to the reduced stunting of VG-20 and VG-21, these accessions also displayed reduced colonization of the race 1 strain JR2, race 2 strains DVD3, DVDS26, DVDS29 and the race 3 strain HOMCF (Fig. 4), confirming that these accessions indeed possess broad-spectrum, race non-specific resistance against $V$. dahliae. VG-20 generally displayed the lowest level of $V$. dahliae colonization, except when inoculated with strain HOMCF of which the colonization in the lower stem was the lowest on VG-21. In contrast to VG-20 and VG-21, on VG-3 only V. dahliae DVDS29 and HOMCF did not display reduced colonization when compared with Moneymaker. Furthermore, of the three wild tomato accessions, the average $V$. dahliae colonization was highest on VG-3 (Fig. 4A-B). 

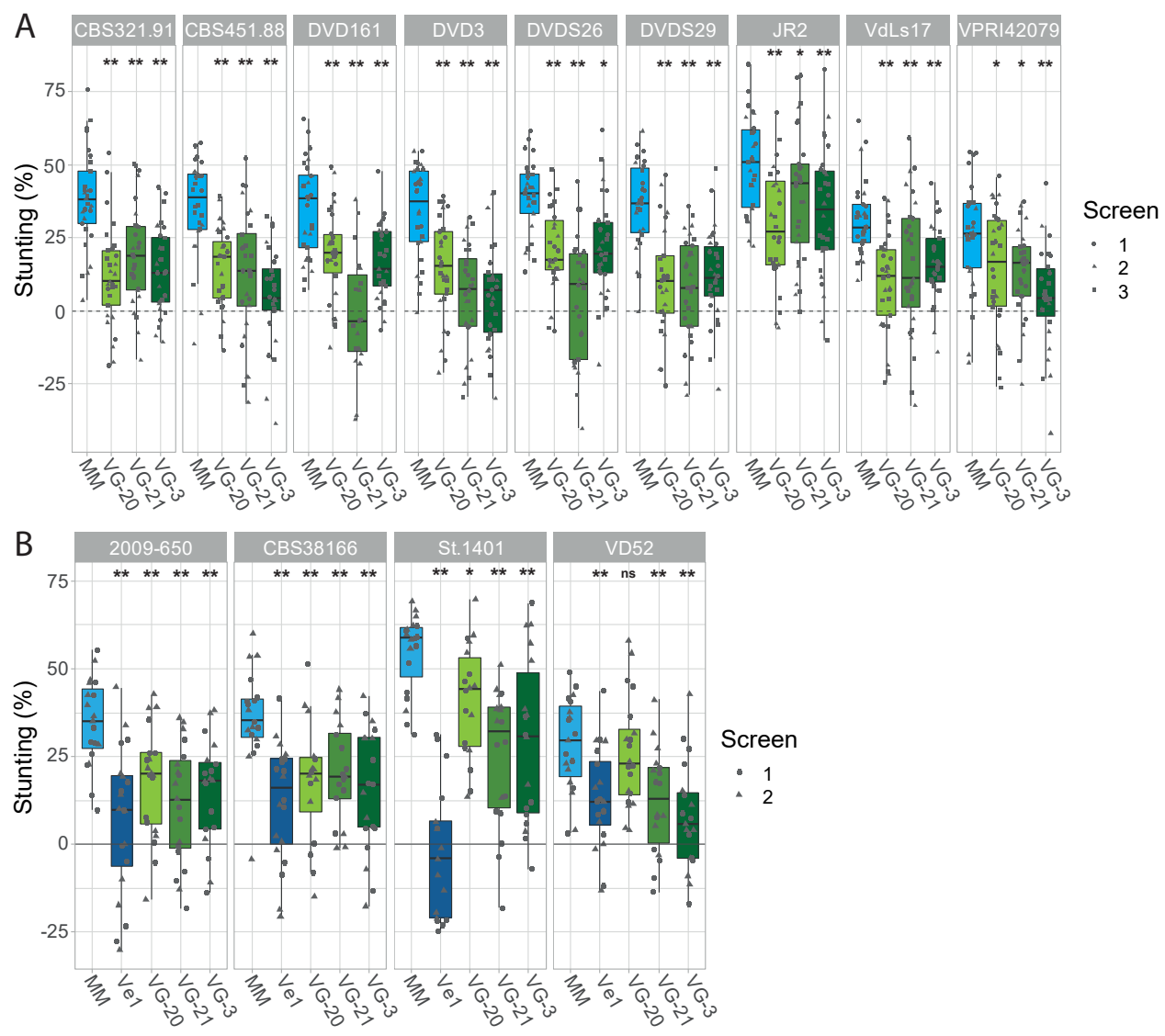

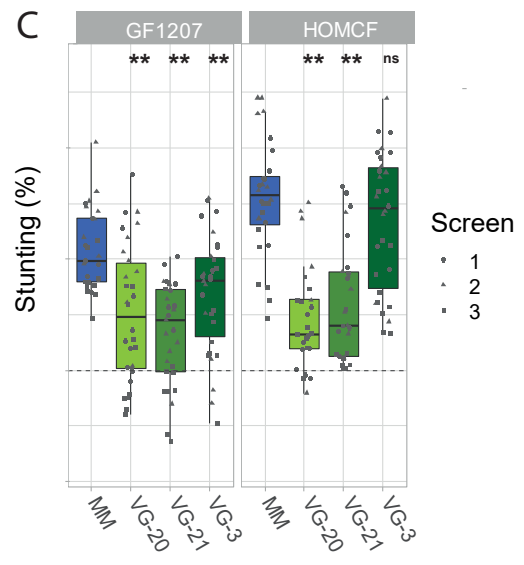

FICURE 3 | Infection screens with a phylogenetically diverse set of Verticillium strains. "MM" refers to S. lycopersicum Moneymaker. VG-20 is of the species S. cheesmanii, VG-21 and VG-3 are S. pimpinellifolium. CBS451.88 is Verticillium nonalfalfa; the other strains belong to Verticillium dahliae. JR2 is a race 1 strain, $\mathrm{HOMCF}$ a race 3 strain, while the remaining strains belong to race 2 . The Y-axis indicates the stunting (\%) of inoculated plants at $21 \mathrm{dpi}$. Panels A-C display results from 3 separate experiments where different shapes of the scattered dots represent separate experiments. Asterisks indicate significant differences in stunting when compared with the stunting of Moneymaker; ${ }^{*} \mathrm{p}<0.05,{ }^{* *} \mathrm{p}<0.005$. Significances were determined in R using a one-way ANOVA followed by a Fishers' LSD test. 

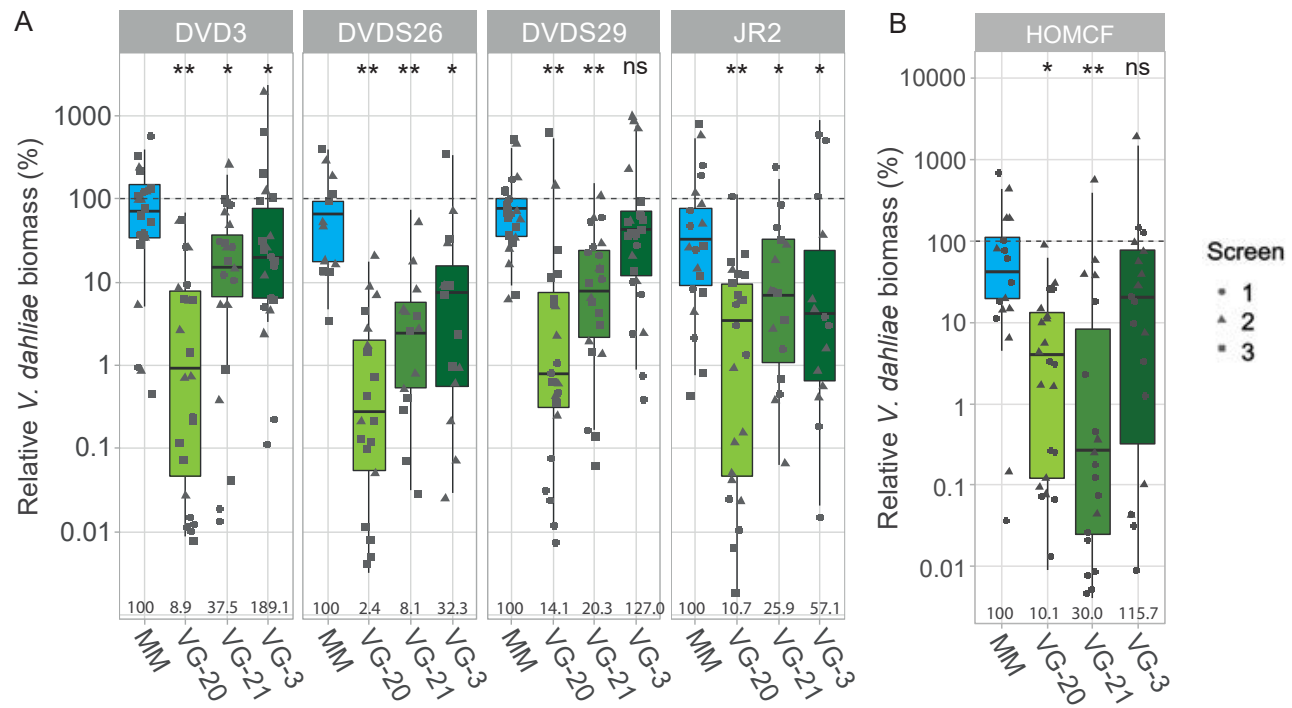

FIGURE 4 | Biomass of V. dahliae in wild tomato accessions VG-20, VG-21 and VG-3 relative to the biomass of V. dahliae in S. lycopersicum Moneymaker (MM). VG-2O is of the species $S$. cheesmanii, VG-21 and VG-3 are $S$. pimpinellifolium. Biomass was determined in lower parts of stems of the plants of which the stunting symptoms are displayed in figure $3 \mathrm{~A}$ and $3 \mathrm{C}$. Biomass of $\mathrm{V}$. dahliae DVD3 and DVDS29 was determined in all three repeat screenings, while the biomass of V. dahliae DVDS26, JR2, HOMCF and VdLs17 in was determined in two repeat screenings. Asterisks indicate significant differences when compared with Moneymaker. Significance levels were determined by a one-way ANOVA followed by a fishers LSD; ${ }^{*} \mathrm{p}<0.05,{ }^{* *} \mathrm{p}<0.005$. Numbers under each boxplot indicate the average relative $V$. dahliae biomass when compared with Moneymaker.

\section{Discussion}

Since the emergence of VeI-resistance overcoming $V$. dahliae race 2 strains, multiple researchers have focused on the identification of additional resistance sources to control $V$. dahliae in tomato. Most resistance sources in tomato against $V$. dahliae described to date are qualitative resistances, such as the resistances governed by Ve1 and V2 (Kawchuk et al. 2001; Fradin et al. 2009; Stamova 2006; Usami et al. 2017).

Although race 1 and 2 strains can be controlled by the introgression of Ver and V2, respectively, no $R$ genes are currently described which control race 3 strains. Therefore, additional resistance sources are required to control $V$. dahliae. In addition to the resistance provided by $V e 1$ and $V 2$, multiple wild tomato accessions which displayed resistance against race 2 strains of $V$. dahliae have been reported (Yadeta 2012). Interestingly, the resistance of these tomato accessions was reported to be effective against race 2 strains but not race 1 strains, as the accessions developed Verticillium wilt symptoms when challenged with two race 1 strains. Possibly, this was because race 2 strains are generally less virulent than race 1 strains, since they lack the Ave1 effector (de Jonge et al. 2012; Yadeta 2012). 
In this chapter, we further evaluated the resistance of a selection of previously described wild tomato accessions (Yadeta 2012). We confirmed that six accessions displayed reduced stunting symptoms when challenged with at least one $V$. dahliae race 2 strain. Of these accessions, VG-3, VG-20 and VG-21 displayed a reduction in stunting symptoms when challenged with a diverse selection of $V$. dahliae race 2 strains. Similar to the observations of Yadeta (2012), the accessions displayed stunting symptoms when challenged with the two aggressive $V$. dahliae race 1 strains JR2 and St.1401. However, the symptom expression was lower than on Moneymaker. Furthermore, when challenged with the less aggressive race 1 strain 2009-650, all three wild accessions displayed a similar symptom expression as when challenged with $V$. dahliae race 2 strains. This all indicates that the resistance of VG-3, VG-20 and VG-21 is not specific to race 2 strains only, which is confirmed by the reduced colonization of the race 1 strain JR2 on all three accessions (Fig. 4). Additionally, since VG-21 and VG-20 also displayed reduced symptom expression compared with Moneymaker when challenged with race 3 strain HOMCF, the resistance of these accessions does not seem to be restricted to any of the current races of $V$. dahliae.

As earlier mentioned by Dan et al., a reduction or absence of $V$. dahliae symptoms does not necessarily mean that the plant is resistant to V. dahliae (Dan et al. 2001), as the plant may be tolerant to the fungus. Similarly, Mikaberidze et al. recently demonstrated that when Zymoseptoria tritici-infected wheat plants display a reduction in symptom expression, these plants are not always necessarily more resistant to $Z$. tritici (Mikaberidze and McDonald 2020). However, since VG-20 and VG-21 displayed a reduction both in symptom expression and in $V$. dahliae colonization, it can be concluded that these accessions are not tolerant but resistant to $V$. dahliae. For VG-3, the relationship between reduced symptom expression and $V$. dahliae colonization is less clear, as no reduction in $V$. dahliae biomass could be detected in plants challenged with V. dahliae DVDS29 and HOMCF. This could indicate that VG-3 is not resistant, but tolerant to these strains. However, we cannot rule out that VG-3 possesses weak resistance against these $V$. dahliae strains. After all, a large degree of variation was observed in the $V$. dahliae biomass measurements. Given that VG-3 generally displayed the highest amount of $V$. dahliae biomass when compared with VG-21 and VG-20, the smaller reduction in $V$. dahliae biomass in VG-3 may have remained unnoticed.

Currently, we have no data which reveal the underlying genetic and molecular mechanisms of the resistance of VG-3, VG-20 and VG-21. However, based on the spectrum of the resistance, some initial hypotheses about the nature of the resistance can be made. Because qualitative and quantitative resistance are often based on different genetic structures, they commonly display different characteristics. Qualitative $R$ genes act in a gene-for gene manner and only control pathogen strains which possess a specific avirulence factor, like Ve1 resistance is only effective against strains which possess Ave1 which can be detected by the Ve1 receptor (Fradin et al. 2009). In contrast, quantitative resistance often provides a more broad-spectrum resistance than qualitative resistance (St.Clair 2010; Corwin and 
Kliebenstein 2017). Given that the resistance of VG-3 and especially VG-20 and VG-21 seems effective against a diverse selection of $V$. dahliae strains, this suggests that this resistance displays more characteristics of quantitative rather than qualitative $R$ gene resistance. Still, this is only an indirect indication, as, $R$ genes may in some cases also provide a more broad-spectrum resistance (i.e. Song et al. 2003; Van Der Vossen et al. 2003). More broadspectrum $R$ gene resistance can occur when the immune receptor detects avirulence factors which are conserved among many pathogen variants. Therefore, to test the hypothesis that these accessions possess quantitative, not qualitative resistance, it should be assessed how this resistance segregates in a mapping population. Further mapping of QTLs or $R$ genes may then reveal the genetic architecture of the resistance. If any QTLs or $R$ genes would be identified for the resistance of VG-21, VG-20 and VG-3, it should be further tested whether these QTLs or R genes are effective against all Verticillium strains used in this study. After all, we currently cannot rule out that the broad-spectrum resistance observed in this study is the result of multiple separate QTLs or R genes which each control a subset of the Verticillium strains used in this study.

Determination of the genetic nature of the resistance may also help to evolve a hypothesis regarding the potential durability of the quantitative resistance of VG-21, VG-20 and VG-3. As defined by $\mathrm{R}$. Johnson, durable resistance is the resistance that remains effective when used in a large growing area for a prolonged period of time in an environment favourable for disease development and spread (Johnson 1981). Compared to qualitative resistance, quantitative resistance is often reported to be more durable (Corwin and Kliebenstein 2017; St.Clair 2010; Parlevliet 2002). Because of its partial and polygenic nature, the fitness benefit for a pathogen of overcoming a gene for partial quantitative resistance can generally be expected to be smaller than that of a strain which overcomes a single strong qualitative $R$ gene. If the resistance of VG-21, VG-20 and especially VG-3 would be quantitative and based on multiple small-effect loci, it thus has the hypothetical potential to be more durable than i.e. V2 resistance. However, if the fitness cost of losing the avirulence factor that is recognized by an $R$ gene is substantial, $R$ gene resistance can also be relatively durable. Given that the Ave1 effector which is recognized by VeI has an important contribution to virulence (de Jonge et al. 2012), it is therefore difficult to predict whether the quantitative resistance of VG-21, VG-20 and VG-3 may turn out to be more durable than Ve1 resistance. Finally, as demonstrated by Pilet-Nayel et al. (2017), especially when a partial, broad-spectrum form of resistance like the resistance of VG-21 and VG-20 is combined with qualitative $R$ genes like $V e 1$ and $V 2$, durable resistance may be achieved. Introgressing the resistance of these wild accessions in lines which also possess VeI and V2 may therefore be the best strategy to use these resistances for the development durable resistance against $V$. dahliae. 


\section{Appendix}

\section{Protocol for DNA isolation of Verticillium-infected tomato stems}

To collect the samples:

1. Harvest the lowest part from the tomato stem; from the soil upwards approx. $3-5 \mathrm{~cm}$

2. Place the stem in a pre-cooled $13 \mathrm{ml}$ tube, and put in liquid nitrogen.

3. Open the tubes, and place then for at least 2 days in the freeze-dryer. After freezedrying, the stems can be stored in the dark at room temperature

4. Before grinding, re-freeze the stems in liquid nitrogen. Smash the stems in the tubes to a rough powder with a metal rod

5. Put the a few scoops of the smashed material in $2 \mathrm{ml}$ Eppendorf tubes. These tubes should at most be filled until 5ooul. If the stems are small (e.g. with wild accessions), you might need to use the whole stem in 1 tube.

6. Add 3 metal balls in each Eppendorf tube.

7. Grind with the RETCH/tissue-lyser for $\sim 30$ seconds. Put the tubes back in the liquid nitrogen, keep frozen.

8. Repeat step 7.

DNA isolation day:

1. Turn the heat block on and set it to $65^{\circ} \mathrm{C}$

2. Prepare $70 \%$ ethanol

3. Place the isolation buffer in the water bath for about $30 \mathrm{~min}$ (pipetting becomes easier)

4. Place isopropanol in the freezer

Work in the fume hood for these steps:

1. Add $\sim 700 \mu \mathrm{L}$ of isolation buffer to each tube containing the already grinded and frozen samples.

2. Vortex the tubes thoroughly

3. Use the thermomixer at $65^{\circ} \mathrm{C}$. Set the thermomixer to shake at $\sim 600 \mathrm{rpm}$ to mix the samples properly. Let samples there for about 1 hour.

(meanwhile prepare the new $2 \mathrm{ml}$ and $1.5 \mathrm{~mL}$ Eppendorf tubes that will be used next).

4. (Without centrifuging): add $\sim$ ImL of chloroform : isoamyl alcohol (24:1)

5. Shake the tubes for $\sim 30$ seconds (you can use tissue paper over the tubes in case the chloroform leaks).

6. Separate phases by centrifuging at $12.000 \mathrm{~g}$ for $30 \mathrm{~min}$ at $4^{\circ} \mathrm{C}$

7. If the water phases appear clean and transparent, continue with step 11. If the samples are brown/dark-coloured/cloudy, continue with step 8.

8. Pipette the water phase in a new $2 \mathrm{ml}$ tube. Add $\sim 1 \mathrm{~mL}$ of chloroform : isoamyl alcohol $(24: 1)$ 
9. Shake the tubes using two tube racks for $\sim 1$ minute (use a tissue paper for the tubes in case the chloroform leaks).

10. Separate phases by centrifuging at $12.000 \mathrm{~g}$ for $10 \mathrm{~min}$ at $4^{\circ} \mathrm{C}$

11. Pipette the water phase (max 600ul) in the new $1.5 \mathrm{~mL}$ tube

12. Add 1 volume of pre-cooled isopropanol to the water phase.

13. Mix the content carefully by inverting it about 10 times, then pellet the DNA by centrifuging for 5 minutes at 12,000 g at 4C. Discard the supernatant (using a pipette)

14. Add $1 \mathrm{~mL}$ of $70 \%$ ethanol (you can stop in this step if needed), and centrifuge for 5 minutes at $12.000 \mathrm{~g}$ at $4 \mathrm{C}$. Discard the supernatant (carefully, using a pipette)

15. Let the pellet dry until it has a "gel" aspect

16. Dissolve the pellet in 100ul TE. It is best to let the pellet slowly dissolve overnight in the fridge.

17. Check the quality of the samples with nanodrop.

\section{Lysis buffer for $500 \mathrm{ml}$}

$\begin{array}{ll}\text { Tris- } \mathrm{HCl} 1 \mathrm{M}(\mathrm{pH} 7.5) & 100 \mathrm{ml} \\ \text { EDTA 0.5 M (pH 8.0) } & 5 \mathrm{ml} \\ \mathrm{NaCl} 5 \mathrm{M} & 200 \mathrm{ml} \\ \mathrm{MQ} & 195 \mathrm{ml} \\ \mathrm{CTAB} & 10 \mathrm{~g}\end{array}$

\section{Extraction buffer for $500 \mathrm{ml}$}

$\begin{array}{ll}\text { Tris-HCl 1M (pH 7.5) } & 50 \mathrm{ml} \\ \text { EDTA 0.5 M (pH 8.0) } & 5 \mathrm{ml} \\ \text { MQ } & 445 \mathrm{ml} \\ \text { Sorbitol } & 31.9 \mathrm{~g} \\ \text { Sarkosyl } & 10 \%\end{array}$

Isolation buffer for $200 \mathrm{ml}$

Lyses buffer

Extraction buffer

Sarkosyl 10\%

RNase A (20mg/ml)

B-mercaptoethanol

TE buffer (pH 7.5)

$1 \mathrm{M}$ Tris- $\mathrm{HCl} \mathrm{pH} 84 \mathrm{ml}$

$0.5 \mathrm{M}$ EDTA

MQ
$105.3 \mathrm{ml}$

$84.2 \mathrm{ml}$

$10.5 \mathrm{ml}$

$20 \mathrm{ul}$

$1 \%$

$400 \mathrm{ml}$ :

$0.8 \mathrm{ml}$

$395.2 \mathrm{ml}$ 


\title{
Chapter 4
}

Mapping of Verticillium dahliae race 2 resistance reveals multiple QTLs from susceptible Solanum lycopersicum Moneymaker, but not from resistant Solanum pimpinellifolium VG-3 and VG-21

\author{
Jasper P. Vermeulen ${ }^{1,2}$, \\ Bart P.H.J. Thomma ${ }^{1,3}$, \\ Yuling $\mathrm{Bai}^{2}$, \\ Henk J.Schouten ${ }^{2}$
}




\section{Abstract}

Most QTL mapping studies to identify plant resistance against Verticillium dahliae report quantitative resistance based on multiple loci. Tomato seems to be an exception, as several sources of monogenic resistance have been reported. However, this resistance often remains only effective towards a subset of $V$. dahliae strains. Therefore, new resistance sources are desirable. We previously identified two accessions, Solanum pimpinellifolium VG-3 and VG21 , that displayed reduced stunting symptom and $V$. dahliae colonization when challenged with a diverse set of $V$. dahliae strains. To study the genetics of this resistance, we screened RIL populations of VG-3 and VG-21 for resistance against race 2 of $V$. dahliae. The segregation of stunting symptom of $V$. dahliae-inoculated plants indicates that both accessions possess quantitative resistance against $V$. dahliae. Unfortunately, no QTLs could be identified based on the stunting symptom in the VG-21 and VG-3 RIL populations. Intriguingly, the VG-3 population also segregated for the yellowing of leaves of $V$. dahliae-inoculated plants, which displayed a different segregation pattern than the stunting symptom. In contrast to the situation as observed for stunting, both the V. dahliae-inoculated $\mathrm{F} 1$ and many of the RILs developed more yellowing leaves than both parents, suggesting that heterozygosity and transgressive segregation might have caused yellowing symptoms on $V$. dahliaeinoculated plants. Since the resistant VG-3 plants did not display less yellowing leaves than the susceptible parent Solanum lycopersicum Moneymaker, this suggests that in the VG-3 population a reduction of the yellowing symptoms may not be associated with resistance but with tolerance to $V$. dahliae. Based on the yellowing symptoms, two QTLs from the susceptible parent Moneymaker could be identified. Unfortunately, however, no QTLs that explain the resistance of VG-3 and VG-21 could be identified. 


\section{Introduction}

The tomato originates from the Andean region in western South America (Blanca et al. 2012; Bai and Lindhout 2007; Bergougnoux 2014). Although the time and place of domestication of the tomato are not precisely known, recent studies suggest that the pre-domesticated tomato from the Andean region was domesticated in Mesoamerica and was subsequently introduced into Europe by the Spanish, after which it spread throughout the world (Bergougnoux 2014; Bai and Lindhout 2007). During the $19^{\text {th }}$ century, the domestication of the tomato as an edible vegetable intensified, and today it is, after potato, the most produced vegetable crop in the world ${ }^{1}$. In the last half-century, average global tomato yields (hg/ha) have increased dramatically with around $300 \%^{1}$. Despite these impressive yield increases, several challenges remain in tomato production. Because the tomato is susceptible to over 200 pests and pathogens (Jones 1991), one of the largest challenges in tomato production is disease management. In tomato cultivation, plant pathogens have the potential to cause average yield losses of over 19\%. Current control practices keep these losses minimized to around $11.3 \%$ globally (Zalom 2003). In agriculture in general, one of the primary approaches to control pests and pathogens has been the use of chemical control agents (Oerke 2006). However, since a few decades, the use of chemicals in agriculture has become severely restricted, particularly in Europe (Lamichhane et al. 2016). With increasing restrictions for the use of agrochemicals, but also keeping increasing resistance development against agrochemicals in pests and pathogens in mind, an important focus of tomato breeders is to breed for resistance against pest and pathogens (Bai and Lindhout 2008, 2007).

Plant resistance can be classified into qualitative and quantitative resistance. Qualitative resistance is in most cases based on a single inherited resistancelocus. Populations segregating for qualitative resistance produce discrete classes of resistant and susceptible individuals. Quantitative resistance, on the other hand, segregates into a continuous distribution of phenotypes from susceptible to resistant and is in most cases based on multiple loci (Corwin and Kliebenstein 2017). Qualitative and quantitative resistance should not be confused with full and partial resistance, as the latter definitions solely define the effectiveness of the resistance, while the former refers to the phenotypic distribution of the resistance. Because of the technical complexity of identifying genes responsible for quantitative resistance, relatively little is known about the molecular mechanisms of this class of resistance. Quantitative resistance has not been used as extensively in resistance breeding as qualitative resistance (St. Clair 2010; Corwin and Kliebenstein 2017). With qualitative resistance, breeders can relatively easily select resistant offspring and introgress the resistance, while for quantitative resistance, the genetics need to be studied to be able to effectively do this. It is therefore not surprising that even before the structure of DNA was resolved (Crick and Watson 1953), several resistance genes had already been introduced into commercial cultivars.

1 http://www.fao.org/faostat/en/\#data, data extracted at 19/01/2020 
One example of early success in resistance breeding is the introduction of the Ve-locus into tomato cultivars in the 1950s, which provides resistance against Verticillium dahliae (Schaible et al. 1951; Deseret News and Telegram 1955). V. dahliae is a soil-borne fungus that infects its hosts through the roots and then attempts to enter the xylem. Once in the xylem, it produces conidiospores that spread throughout the plant with the sap stream (Fradin and Thomma 2006). On tomato, common symptoms may include stunting, wilting, yellowing and necrosis of the leaves. Because $V$. dahliae is a vascular pathogen, most fungicides are ineffective once it has entered the plant because the fungus cannot be reached. The fungus produces resting structures called microsclerotia which can remain viable in the soil for many years (Wilhelm 1955). This combined with its broad host range, which comprises hundreds of plant species, explains why $V$. dahliae remains a challenging pathogen to control, even in the $21^{\text {st }}$ century. Although the introduction of the Ve-locus in commercial tomato cultivars was welcomed by farmers, only a few years after its introduction, $V$. dahliae strains emerged that were able to overcome the resistance of Ve plants and cause disease (Robinson 1957; Alexander 1962). Since then, resistance-overcoming V. dahliae strains were assigned to race 2, whereas strains that remain to be contained by the $V e$-locus are assigned to race 1.

Considerable efforts have been made to identify race 2 resistance in tomato. Whereas most of these efforts have remained unsuccessful, the Japanese rootstock accession $S$. lycopersicum Aibou was reported to display qualitative resistance against race 2 strains. This resistance was found to be based on a single dominantly inherited locus, V2 (Usami et al. 2017). However, no $R$ gene has been mapped yet for this resistance. Importantly, the resistance governed by $V 2$ appears to be effective to only a subset of race 2 strains, as several strains were found to be able to cause severe foliar and vascular symptoms on these plants. Therefore, $V$. dahliae was further classified into three races. According to this latest classification, strains which have overcome $V e 1$ or $V 2$ resistance are classified as race 2 and 3 , respectively. Strains which are contained by $V e 1$ and $V 2$ are then classified as race 1.

To control race 3 strains, but also to provide further resistance against $V$. dahliae in general, novel resistance sources are desirable. In contrast to the qualitative resistance from Ver and $V 2$, studies for $V$. dahliae-resistance in other crops such as cotton, strawberry, eggplant and rapeseed mostly report other quantitative resistance based on multiple QTLs (Rygulla et al. 2008; Antanaviciute et al. 2015; Bolek et al. 2005; Wang et al. 2008; Zhao et al. 2014; Toppino and Barchi 2016).

Previously, a wide collection of wild tomato germplasm was screened for resistance against race 2 of $V$. dahliae (Yadeta 2012). Several of these wild accessions displayed a reduction in $V$. dahliae-associated stunting symptom (Yadeta 2012). In chapter 3, we confirmed the resistance of some of these accessions. In this chapter, we aim to identify QTLs for the resistance of two of these accessions, VG-3 and VG-21, both belonging to the species Solanum pimpinellifolium. 
To this end, a RIL population was developed for each of these accessions based on a cross with the susceptible parent Solanum lycopersicum Moneymaker. Unfortunately, based on the segregation of $V$. dahliae-associated stunting symptom, we were unable to identify QTLs for the resistance of the two $S$. pimpinellifolium accessions. However, in the RIL population of VG-3, we identified two QTLs from the susceptible parent Moneymaker that reduced the number of yellowing leaves in $V$. dahliae-inoculated plants. Moreover, we provide evidence showing that the RIL population of VG-3 segregates differentially for two types of Verticillium wilt symptoms, yellowing and stunting, suggesting that different genetic mechanisms affect the development of these symptoms.

\section{Materials \& methods}

\section{Development of RIL-populations and genotyping}

A previously generated and fully genotyped F7 RIL population of S. lycopersicum Moneymaker $\mathrm{x}$ S. pimpinellifolium VG-3 was available at Wageningen. To produce the RIL-population with S. pimpinellifolium VG-21, S. lycopersicum Moneymaker was used as mother plant. After selfing of F1 plants, seeds of the F2 population were selfed by single seed descent until F6. To genotype the RIL population, leaves were harvested from F5-parental plants. To select SNP markers for genotyping of the RIL population, all markers from Sim et al. (2012) were tested on Moneymaker and VG-21. Based on the tomato EXPMIMP 2009 genetic map (Ashrafi et al. 2009), the genetic positions of the markers were interpolated. Out of all 7,688 SolCapSNPs, 192 polymorphic markers were selected for genotyping the RIL population. The selected markers were distributed across the interpolated EXPIMP 2009 map with intervals of on average $5.5 \mathrm{cM}$.

\section{Marker validation and construction of the genetic map}

For the Moneymaker x VG-3 population, we used the genetic map that was already available at Wageningen University. For the Moneymaker x VG-21 RIL population, we developed a novel genetic map. Before constructing the genetic map, all markers were first visually inspected. Markers for which the genotyping resulted in no data or only heterozygous data were removed. Next, markers were further validated by checking for (1) unusually skewed segregation of markers, (2) markers with $>10 \%$ of missing data, (3) identical markers on different physical positions, and (4) unusually high crossover rates between physically closely located markers, using the R-packages R/QTL and AsMAP (Taylor and Butler 2017). Markers which were considered as unreliable based on these criteria were removed. Using the "MSTmap" function of the R package ASMap (Taylor and Butler 2017), linkage groups were constructed for each chromosome based on the 188 remaining markers with a p-value of $10^{-4}$ with the options "anchor" and "byChr" enabled. The physical order and genetic order 
were evaluated using the "ripple" function of R/QTL. If the genetic order and physical order of markers were not in agreement, the likelihood of both marker orders was estimated using the "ripple" function of the package R/QTL version 1.45-6 (Broman et al. 2003). If the genetic order and physical order of the markers was equally likely, we favoured the physical order of the markers.

\section{Preparation of inoculum and inoculation procedures}

The RIL-population Moneymaker x VG-3 was screened with V. dahliae isolate DVD3, while the RIL-population Moneymaker x VG-21 was screened with V. dahliae isolate DVDS29. To produce the inoculum, five petri dishes with Potato Dextrose Agar were inoculated with the strains from a $-80^{\circ} \mathrm{C}$ glycerol stock.

To produce the inoculum for screening the Moneymaker x VG-3 RIL population, 20 petri dishes with potato dextrose agar were inoculated from the 5 petri dishes with the fungal culture. Five days after inoculation, spores were harvested from the potato dextrose agar, washed and resuspended in tap water to a final concentration of $10^{7}$ conidiospores per $\mathrm{mL}$. For inoculation, 10-day old seedlings were carefully uprooted and roots were washed in tap water. On average, of each RIL 8-10 plants were used of which half were root-dipped for 10 minutes in water and the other half were root-dipped for 10 minutes in the inoculum. After inoculation, seedlings were placed in $14 \mathrm{~cm}$ pots and randomly distributed across the greenhouse compartment.

To produce the inoculum for screening the Moneymaker x VG-21 RIL population, agar blocks of $\sim 1 \mathrm{~cm} 2$ were cut from the 5 petri dishes with the fungal culture and transferred to freshly-made potato sucrose broth that was produced by boiling 400 grams of pre-peeled potato dices in $1 \mathrm{~L}$ of water for 15 minutes. The water in which the potatoes were boiled was then filtered through cheese cloth. Per $\mathrm{L}$ of broth, $1 \mathrm{~L}$ of water and $40 \mathrm{~g}$ of sucrose was added, followed by autoclaving for 30 minutes at $120^{\circ} \mathrm{C}$. After inoculating the broth with the agar blocks, it was kept in an incubator for at least 5 days at $24^{\circ} \mathrm{C}$ at $100 \mathrm{rpm}$. Using Mira cloth, mycelium was filtered out of the broth. Subsequently, the inoculated broth was centrifuged at 3,500 rpm for 30 minutes and the supernatant was removed. Next, the conidiospores were re-suspended in tap water to a final concentration of $10^{7}$ conidiospores $/ \mathrm{mL}$. For inoculation, 12-day-old seedlings were carefully uprooted and the roots were washed in tap water. Of each genotype, on average, 12-14 plants were used per genotype. Half of the plants were mock-inoculated by root-dipping them in water for 10 minutes, whereas the other half were root-dipped for 10 minutes in the inoculum. After inoculation, seedlings were potted into trays of small cups containing sowing soil. From these trays, the cups were transferred to $14 \mathrm{~cm}$ pots according to the randomization scheme described below. Plants in block 1 and 2 were randomized one day after the inoculation, plants in block 3 and 4 two days after inoculation and the plants in block 5 and 6 three days after inoculation. To this end, of 
each genotype, the replicate plants were randomized across the greenhouse compartment in six blocks. The randomization was performed in such a way that the genotypes were represented by one replicate mock- and one replicate $V$. dahliae-inoculated plant in each of the blocks. The remaining plants of each genotype were randomly assigned to one of the blocks. All genotypes were furthermore randomized across the blocks in pairs of mockinoculated and V. dahliae-inoculated plants (Fig. 1). By doing so, of each genotype, a mockinoculated plant was located directly adjacent to a $V$. dahliae-inoculated plant of the same genotype (Fig. 1).
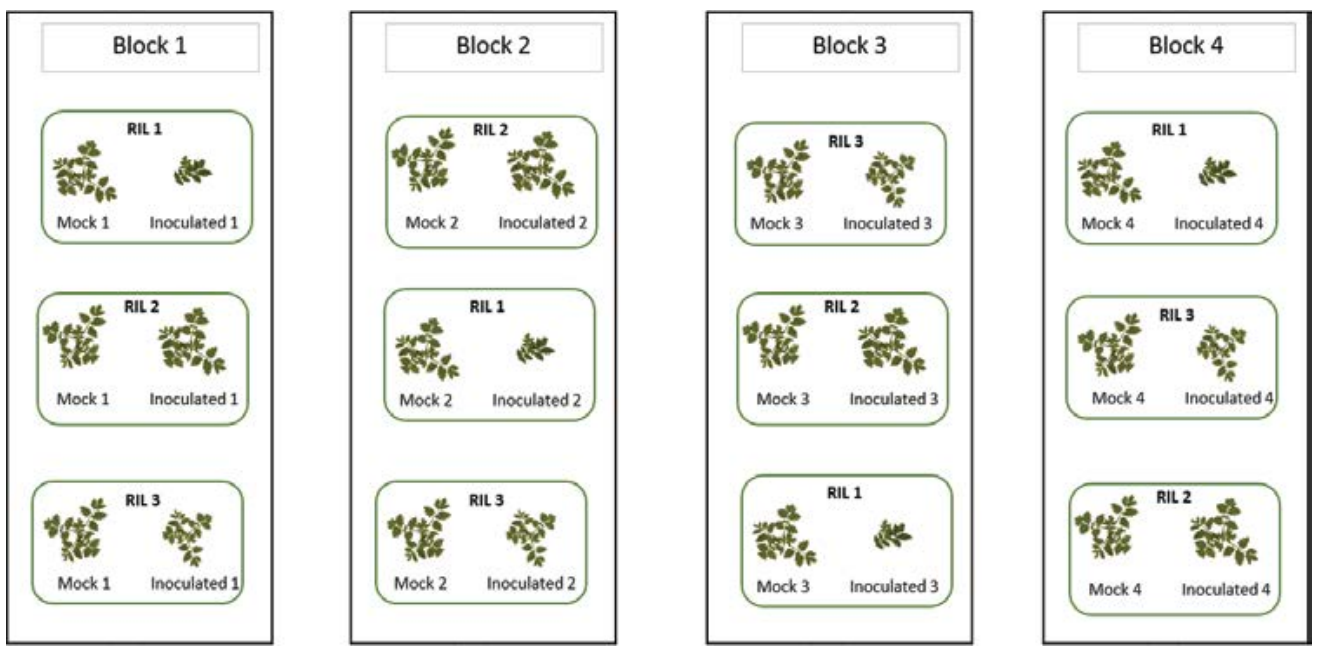

FICURE 1 | Overview of the first four blocks of the paired randomized block design that was used to position the RILs in the greenhouse. RILs were randomized across the greenhouse over six blocks in pairs of mock-treated and inoculated plants such that a mock-treated and inoculated plant are always immediately adjacent.

\section{Phenotyping of the populations}

Plants of the Moneymaker x VG-3 population were phenotyped at 21- and 27-days postinoculation (dpi). At $21 \mathrm{dpi}$, the canopy of individual plants was photographed from the top using a Nikon D3200 DSLR camera that was fixed on a tripod. Using ImageJ version 1.51f (Schneider et al. 2012), the canopy area of the plants was then determined. The degree of stunting of $V$. dahliae-inoculated plants was calculated by comparing the size of the inoculated plant with the average size of all mock-treated plants of the same genotype, using the following formula:

$$
\text { Stunting }(\%)=\left(1-\frac{\text { Canopy area of inoculated plant }}{\text { Average canopy area of all mock }- \text { treated plants of genotype } x}\right) * 100
$$


Furthermore, at $27 \mathrm{dpi}$, the number of leaves which developed yellowing symptoms were counted both for the mock-inoculated and the V. dahliae-inoculated plants.

Plants of the Moneymaker x VG-21 population were phenotyped at 24 and 25 days after inoculation. Experimental blocks 1 to 3 were phenotyped at 24 dpi and blocks 4 to 6 at 25 dpi. The canopy area was determined as described above. The stunting of $V$. dahliae-inoculated plants was calculated by comparing the canopy area of the $V$. dahliae-inoculated plant with the canopy area of mock-inoculated plants within each pair using the following formula:

$$
\text { Stunting }(\%)=\left(1-\frac{\text { Canopy area of inoculated plant of pair } x \text { of genotype } y}{\text { Canopy area of mock-treated plant } x \text { of genotype } y}\right) * 100
$$

To detect outliers, a one-way ANOVA was performed for differences in stunting. Based on the studentized residuals from this analysis, outliers were identified. Datapoints with studentized residuals below -2.5 or above 2.5 were considered as outliers and removed.

The broad-sense heritability was estimated from the output of a one-way ANOVA using the formula below:

$$
\sigma_{e}^{2}=M S_{\text {residual }} \text { and } \sigma_{g}^{2}=\frac{M S_{\text {genotype }}-\sigma_{e}^{2}}{\text { average number of replicates per genotype }}
$$

Estimated broad sense heritability $\left(H^{2}\right)=\frac{\sigma_{g}^{2}}{\sigma_{g}^{2}+\sigma_{e}^{2}}$

\section{QTL analysis in $\mathbf{R}$}

QTL analyses were performed with version $1.45-6$ of the R package R/QTL (Broman et al. 2003). A QTL analysis consisted of 3 steps. First, using the function "scanone", an interval mapping analysis was performed using the "em" algorithm. A permutation test with 1000 permutations was then performed with the "scanone" function to determine the significance threshold ( $\mathrm{p}<0.05$ ). For the QTL analysis, two covariates were added. As the phenotypes from both experiments were included in the same analysis, one covariate was used to separate the phenotypes from both experiments. As a second covariate, the average canopy area of mock-inoculated plants of each RIL was included. After the "scanone" analysis, a two-dimensional QTL scan called "scantwo" was performed using the "em" algorithm. Also, in this case, a permutation test was performed. Due to the high amount of computational time required for this permutation test, the permutations were divided into ten batches using the "n.batch" option and were analysed on a Linux-based server cluster. All evidence for QTLs found with "scanone" and "scantwo" were combined into a multiple QTL model using the function "makeqtl", followed by "fitqtl", and refined with the "refineqtl" function. Using the function "stepwiseqtl", the most likely multiple QTL models were evaluated. 
The significance threshold for this analysis was determined using the permutation test of "scantwo" with the "imp" algorithm.

\section{Results}

To study the segregation of Verticillium wilt symptoms in the Moneymaker $x$ VG-3 and Moneymaker x VG-21 RIL populations, we inoculated the populations with $V$. dahliae race 2 strain DVD3 or DVDS29, respectively. These strains were selected because they displayed on (1) a clear reduction in stunting symptoms and pathogen biomass, and (2) provided a contrast in stunting symptoms between the resistant parent and the susceptible parent (chapter 3 of this thesis); For VG-20, this was V. dahliae strain DVDS29.

\section{QTL analysis of the resistance of VG-3}

Stunting symptom of V. dahliae-inoculated plants segregated quantitatively in the MM $x$ VG-3 RIL population

In the two phenotyping screens of the Moneymaker x VG-3 RIL-population, Moneymaker plants grouped with $37 \%$ stunting and $56 \%$ stunting, respectively, among the genotypes with the highest degree of stunting, confirming its susceptibility to V. dahliae (Fig. 2 A-B). The resistant VG-3 parent displayed no clear stunting symptoms in the two experiments $(-3,9 \%$ and $-3.6 \%$ stunting, respectively), confirming its resistance against $V$. dahliae DVD3. The F1 displayed an intermediate level of symptoms in the first screen (16,6\% stunting), whereas it grouped with VG-3 in absence of stunting symptoms in the second screen (0.8\% stunting). This suggests that the resistance inherits - at least partially - in a dominant fashion. As the RILs segregated as a continuum from susceptible to resistant phenotypes (Fig. 2), this suggests that VG-3 possess quantitative resistance against $V$. dahliae, most likely based on multiple genes.

As Verticillium wilt symptoms typically display a considerable degree of variation, we estimated both the heritability and reproducibility of the stunting symptom of $V$. dahliaeinoculated plants (Table 1 ). An estimated heritability of $H^{2}=0.34$ and $H^{2}=0.49$ was obtained for the first and second screen, respectively. The correlation coefficient $\left(R^{2}\right)$ was only 0.04 between the two experiments. Eleven of the RILs displayed differences of more than $25 \%$ stunting between the two experiments (Fig. 3). Exclusion of these genotypes from the dataset resulted in an increase in reproducibility to $R^{2}=0.22$ (Table 1 ). Furthermore, removal of these RILs resulted in a slight reduction in the estimated heritability in the individual experiments. In the combined two experiments, the removal resulted in a slight increase in the estimated heritability from $H^{2}=0.27$ to $H^{2}=0.32$ (Table 1 ). 

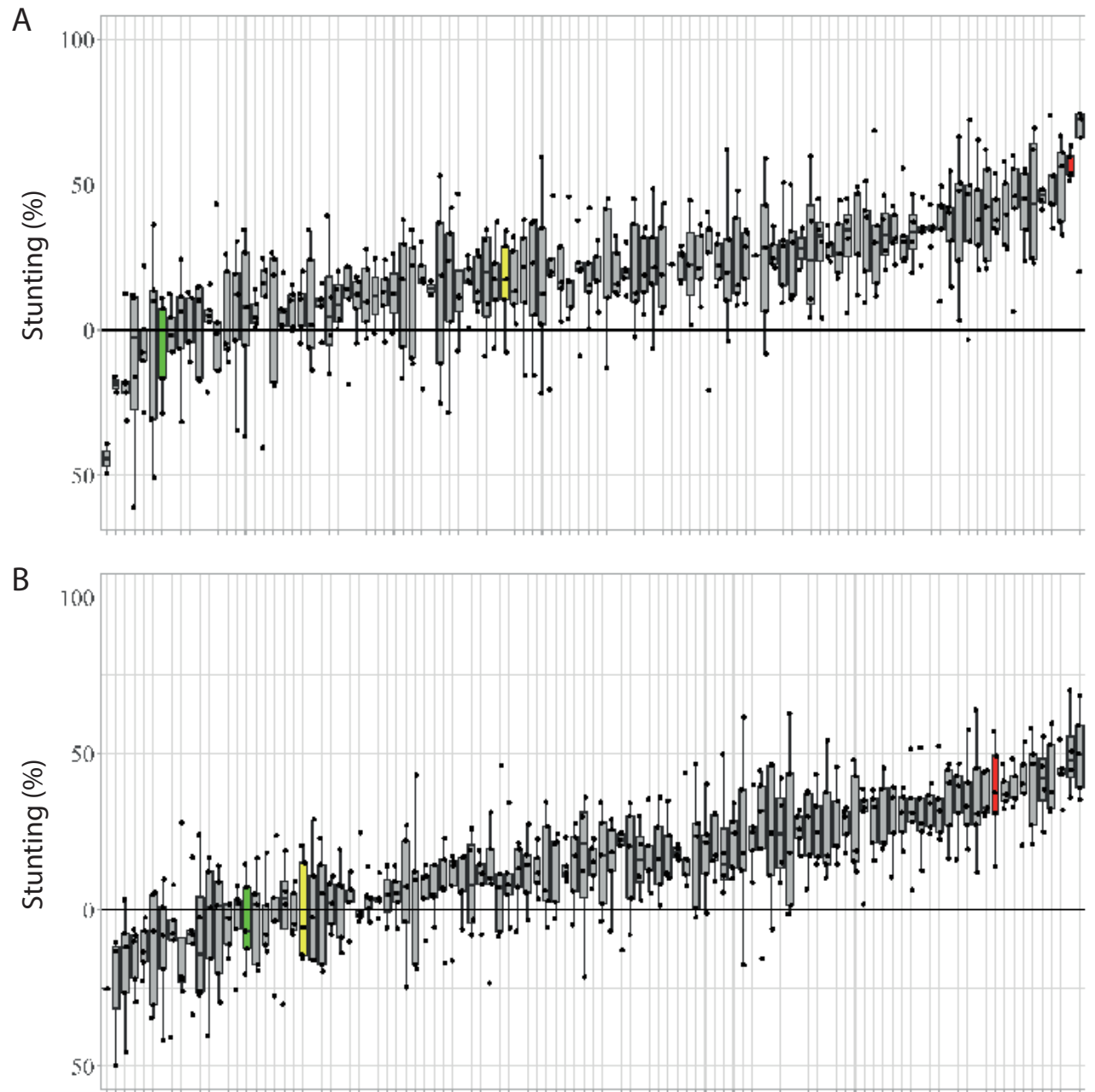

FICURE 2 | Stunting (\%) of the S. lycopersicum Moneymaker parent (red), the S. pimpinellifolium VG-3 parent (green), the $\mathrm{Fl}$ (yellow), and the RILs of the S. lycopersicum Moneymaker x S. pimpinellifolium VG-3 population (grey) at 21 days after inoculation with $V$. dahliae strain DVD3, from the first (A) and second (B) screen. Stunting reflects the relative reduction in canopy area of $\mathrm{V}$. dahliae-inoculated plants relative to the canopy area of mock-inoculated plants of the same genotype. 


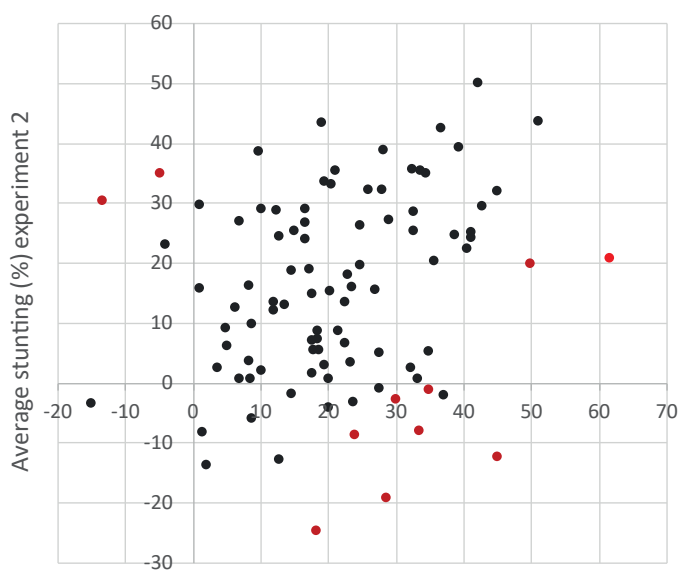

Average stunting (\%) experiment 1
FICURE 3 Comparison of the average stunting of $V$. dahliae-inoculated RILs of the $S$. lycopersicum Moneymaker x $S$. pimpinellifolium VG-3 RIL-population (individual dots) in the first experiment ( $x$-axis) and the second experiment (y-axis). Red dots represent 11 RILs with the largest variability in phenotype between the two experiments.

TABLE 1 | Heritability and correlation estimates

\begin{tabular}{|c|c|c|c|c|}
\hline $\begin{array}{l}\text { Heritability }\left(H^{2}\right) \text { and correlation } \\
\text { coefficient }\left(R^{2}\right)\end{array}$ & $H^{2}$ experiment 1 & $H^{2}$ experiment 2 & $\begin{array}{c}H^{2} \text { experiment } 1+2 \\
\text { combined }\end{array}$ & $\begin{array}{c}R^{2} \text { between } \\
\text { experiment } 1 \& 2\end{array}$ \\
\hline All RILs & 0.34 & 0.49 & 0.27 & 0.04 \\
\hline $\begin{array}{l}\text { Only RILs with the most } \\
\text { consistent phenotype }\end{array}$ & 0.31 & 0.46 & 0.32 & 0.22 \\
\hline
\end{tabular}

$H^{2}$ and $R^{2}$ refer to the estimates of the broad-sense heritability $\left(H^{2}\right)$. and Pearson correlation coefficient $\left(R^{2}\right)$ of the stunting of $V$. dahliae-inoculated plants in the Moneymaker x VG-3 RIL-population, with either all RILs or only the RILs with the most consistent phenotype.

\section{No QTLs were identified in the VG-3 population for stunting}

Given that the stunting symptom represents the effect of $V$. dahliae inoculation on the growth of the plant, segregation in plant size might affect the stunting symptom. The RILpopulation segregated for the canopy area of mock-inoculated plants, for which we mapped a QTL on chromosome 1 using interval mapping (Fig. S1). We observed a low but significant correlation between the size of mock-inoculated plants and the stunting of $V$. dahliaeinoculated plants. It thus appears that larger plants were slightly more stunted (Fig. S2). Therefore, we considered the canopy area of mock-inoculated plants as a covariate in the QTL analysis of stunting. For the QTL analysis, we used the R package R/QTL (Broman et al. 2003; Broman and Sen 2009) and used both interval mapping and a two-dimensional twoQTL scan called "scantwo" to search for QTLs. Unfortunately, with none of the methods we could detect QTLs that displayed LOD-values above the thresholds set by the permutation tests. Also, when the eleven RILs with the largest differences in phenotype between the two experiments (Fig. 3) were removed, no significant QTLs could be detected. 
The MM x VG-3 RIL population segregated transgressively for the number of yellowing leaves in V. dahliae-inoculated plants

Besides for stunting, the Moneymaker x VG-3 RIL population also segregated for the yellowing of leaves in $V$. dahliae-inoculated plants (Fig. 4). Although a small number of mock-inoculated plants also displayed some yellowing of leaves, no meaningful correlation between the yellowing of leaves in mock-inoculated and $V$. dahliae inoculated plants was observed $\left(R^{2}=0.063\right.$, Fig. S3). This thus indicates that the yellowing of leaves in $V$. dahliaeinoculated plants was caused by the $V$. dahliae inoculation.

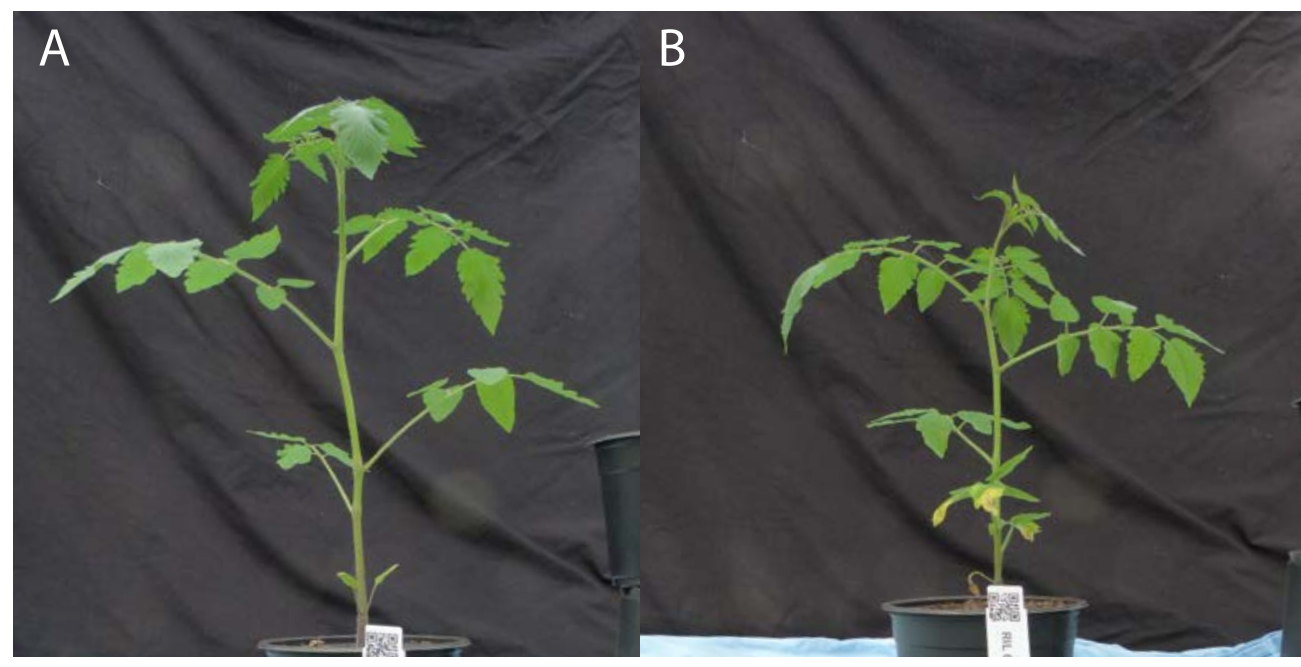

FICURE 4 | Example of yellowing observed on the lower leaves of a V. dahliae DVD3-inoculated RIL (B), which was not visible in the mock-treated plants (A). The pictures were taken at 27 days after inoculation with $V$. dahliae.

No meaningful correlation $\left(R^{2}=0.02\right)$ was found between the stunting and the number of yellowing leaves of $V$. dahliae inoculated plants (Fig. S4). Furthermore, although Moneymaker plants were clearly more stunted than VG-3 plants, Moneymaker plants did not display more yellowing leaves than VG-3 plants (Fig. 5). This all demonstrates that the yellowing symptoms segregate differently in the Moneymaker x VG-3 RIL population than the stunting symptom. In fact, many $V$. dahliae-inoculated RILs displayed a higher number of yellowing leaves than both parental lines (Fig. 5). These results demonstrate that the yellowing symptoms of $V$. dahliae-inoculated plants segregate transgressively in this population. Furthermore, also the $V$. dahliae-inoculated Fi plants displayed a higher degree of yellowing symptoms than both parents, indicating that yellowing symptoms are more profound in heterozygous plants.

Although we did not observe segregation for the total number of leaves in the Moneymaker $\mathrm{x}$ VG-3 RIL population, the population did segregate for plant size. To test whether 
larger plants displayed more yellowing, i.e. due to nutrient deficiencies, we performed a correlation analysis. No meaningful correlation $\left(R^{2}=0.01\right.$, Fig S6) was found between the number of yellowing leaves of $V$. dahliae-inoculated plants and the canopy area of mockinoculated plants.

The estimated heritability of the yellowing symptoms was similar to the estimated heritability of the stunting of $V$. dahliae-inoculated plants $\left(H^{2}=0.38\right.$ vs $\left.H^{2}=0.32\right)$ (Table 1-2). However, the reproducibility of the yellowing symptoms was higher than that of the stunting, with $R^{2}=0.48$ (Fig. 6) for the number of yellowing leaves versus $R^{2}=0.22$ (Fig. 3) for stunting.

A

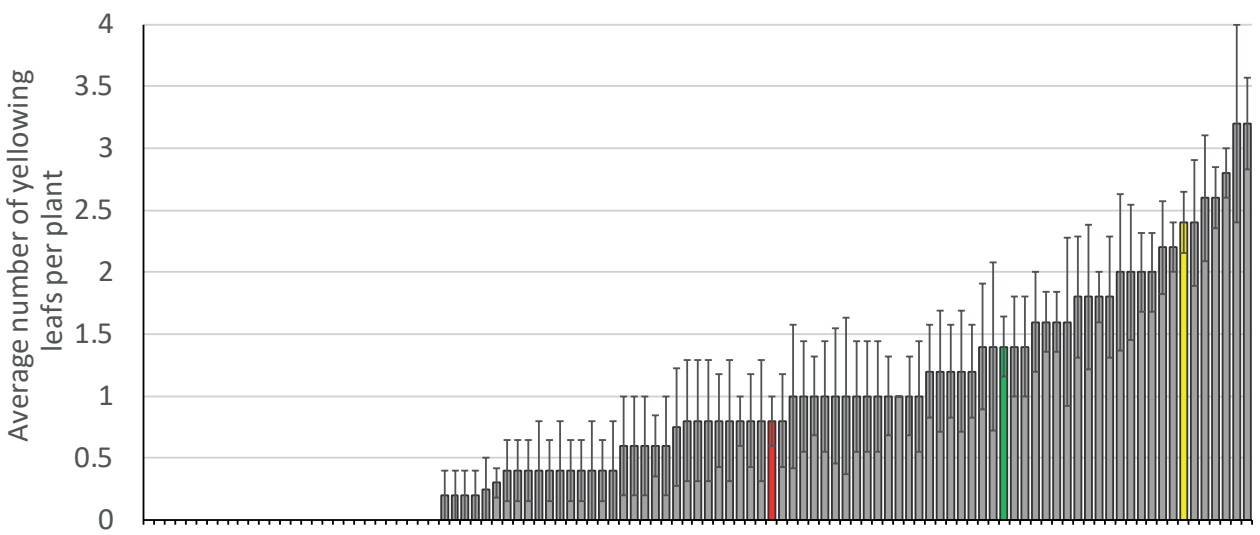

B

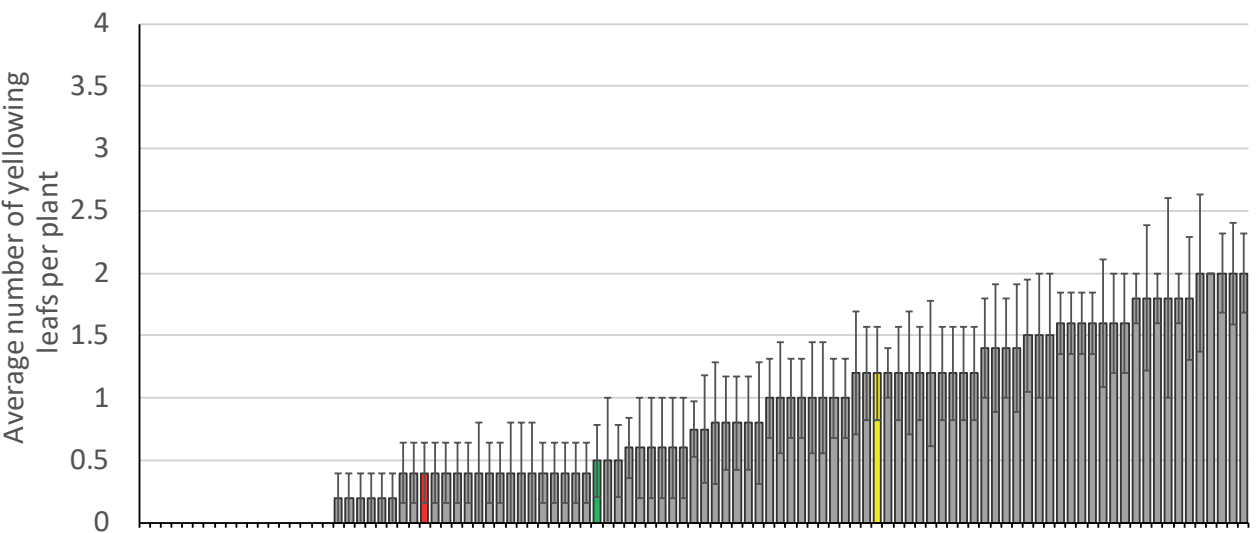

FICURE 5 | Average number of yellowing leaves of $V$. dahliae DVD3-inoculated plants of the S. lycopersicum Moneymaker parent (red), the $S$. pimpinellifolium VG-3 parent (green), the F1 (yellow), and the RILs of the S. lycopersicum Moneymaker x S. pimpinellifolium VG-3 population (grey) at 23 days post-inoculation, from the first (A) and second (B) screen. 
TABLE 2 | Heritability estimates.

\begin{tabular}{cccc} 
& Experiment 1 & Experiment 2 & Experiment 1 + 2 \\
\hline$H^{2}$ & 0.46 & 0.38 & 0.38 \\
\hline
\end{tabular}

$H^{2}$ refers to the estimated broad-sense heritability of the number of yellowing leaves of $V$. dahliae-inoculated plants. Heritability was estimated using a one-way ANOVA.

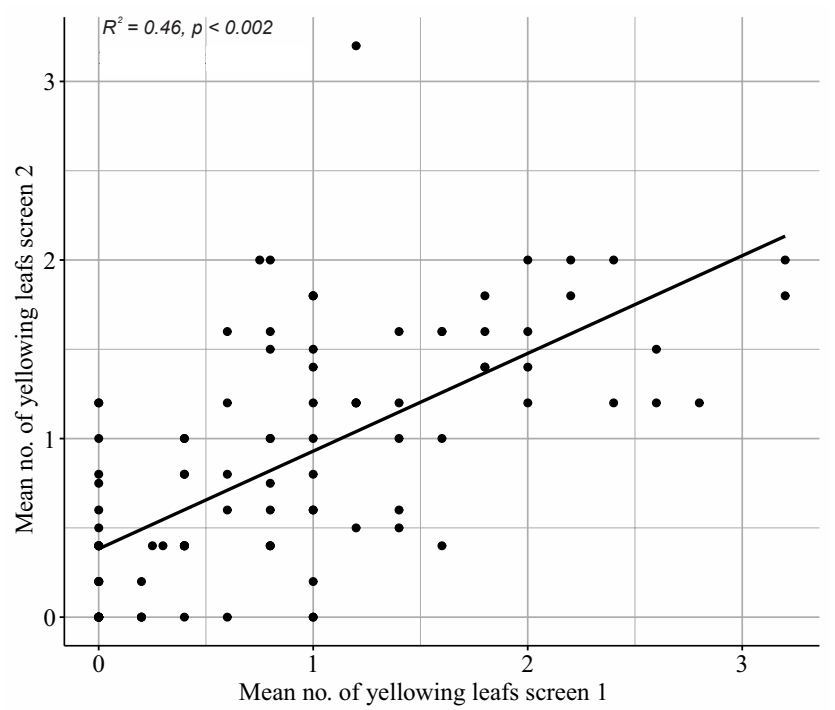

FIGURE 6 | Spearman's correlation for the average number of yellowing leaves of $V$. dahliae-inoculated RILs of the S. lycopersicum Moneymaker x S. pimpinellifolium VG-3 population between the first screen ( $\mathrm{x}$-axis) and the second screen (y-axis). Correlations were estimated using the nonparametric Spearman's rank correlation test.

\section{Two QTLs in the VG-3 RIL population were associated with yellowing of V. dahliae-inoculated plants}

To search for QTLs associated with the number of yellowing leaves of $V$. dahliae-inoculated plants, we used the same approach as described above for the stunting. Some of the RILs also displayed a low number of yellowing leaves of mock-treated plants. To take this into account in the QTL analysis, we included the number of yellowing leaves of mock-treated plants as a covariate. With this approach, we found strong evidence for a major QTL on cho3 (Fig. S9A). This approach also revealed some evidence for epistasis between this QTL on cho3 and another QTL on cho5 (Fig. S9B). Besides these two QTLs, some indications for additional QTLs on ch04, cho8, and ch12 were found (Fig. S9A). To determine which combination of all found QTLs is best supported by the data, we performed a stepwise selection method called "stepwiseqtl". The stepwiseqtl analysis determined that a model consisting of the QTLs on cho3 and cho5 is best supported by the observed data (Fig. 7, Table 3). 
For both QTLs, RILs with the Moneymaker allele displayed fewer yellowing symptoms than RILs with the VG-3 allele (Fig. 8A). However, in agreement with the found epistasis between the QTLs on ch03 and ch05, the QTL from Moneymaker on cho5 only affected the yellowing symptoms in RILs which have the VG-3 allele of the QTL on cho3 (Fig. 8B). Together, the QTLs explained $27.6 \%$ of the phenotypic variation, with the QTL on cho3 explaining $20.6 \%$, and the QTL on ch05 7.0\%, respectively. The covariates explained around 10\% of the phenotypic variability. This demonstrates that including the yellowing symptom of mock-inoculated plants reduced the residual variation in the analysis, thereby justifying the inclusion of the covariate. Finally, although a few RILs also displayed yellowing in mock-inoculated plants, no QTLs were found based on the number of yellowing leaves of mock-inoculated plants (Fig. S9).

TABLE 3 | The most likely QTL model for V. dahliae-associated yellowing symptoms

\begin{tabular}{|c|c|c|c|c|c|c|c|}
\hline Chromosome & 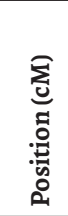 & 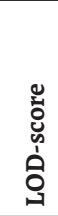 & 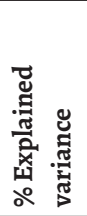 & 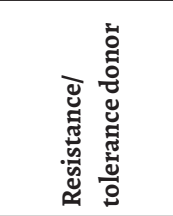 & 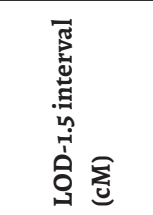 & 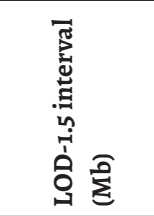 & 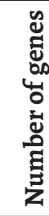 \\
\hline 3 & 96 & 11.3 & 20.6 & Moneymaker & $\begin{array}{c}30.5 \\
(73.6-104.1)\end{array}$ & $\begin{array}{c}11.9 \\
(1.06-46.5)\end{array}$ & 279 \\
\hline 5 & 26.4 & 4.2 & 7.0 & Moneymaker & $\begin{array}{c}45.4 \\
(1.06-46.5)\end{array}$ & $\begin{array}{c}4.6 \\
(0.42-5.02)\end{array}$ & 521 \\
\hline
\end{tabular}

The QTLs for yellowing of leaves of $V$. dahliae-inoculated plants in the S. lycopersicum Moneymaker x S. pimpinellifolium VG-3 RIL population which are best supported by the data. The number of genes was estimated based on the physical positions of the flanking markers of the QTLs on the reference genome Heinz SL2.50. Annotated genes were based on the ITAG annotations version 2.4.

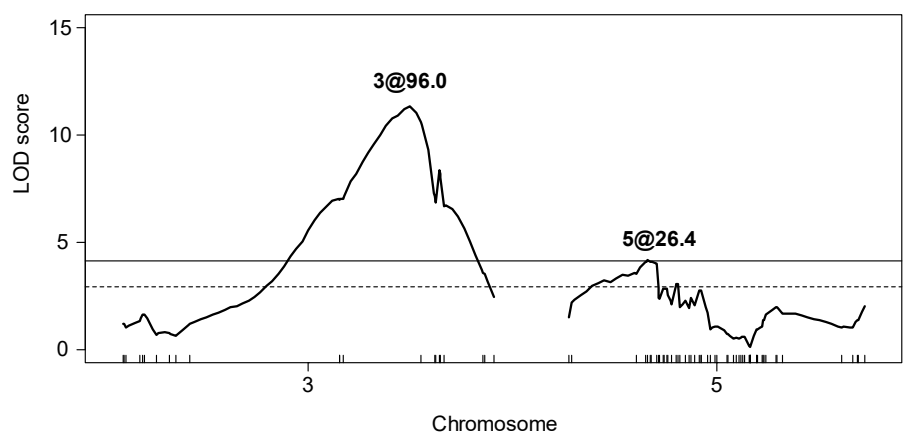

FIGURE 7 | LOD-profile of the most likely multiple QTL model with the QTLs on ch03 and cho5 for the number of yellowing leaves of $V$. dahliae-inoculated plants. Y-axis represents the LOD-score of the QTLs. Vertical bars on the $\mathrm{x}$-axis display the genetic position (cM) of the genetic markers on cho3 and 5 . The horizontal dashed line represents the main-effect penalized LOD-threshold, the horizontal solid line represents the strict interaction penalized LOD-threshold. Thresholds were determined by a two-dimensional permutation test ( $\mathrm{n}=1000$ ). 

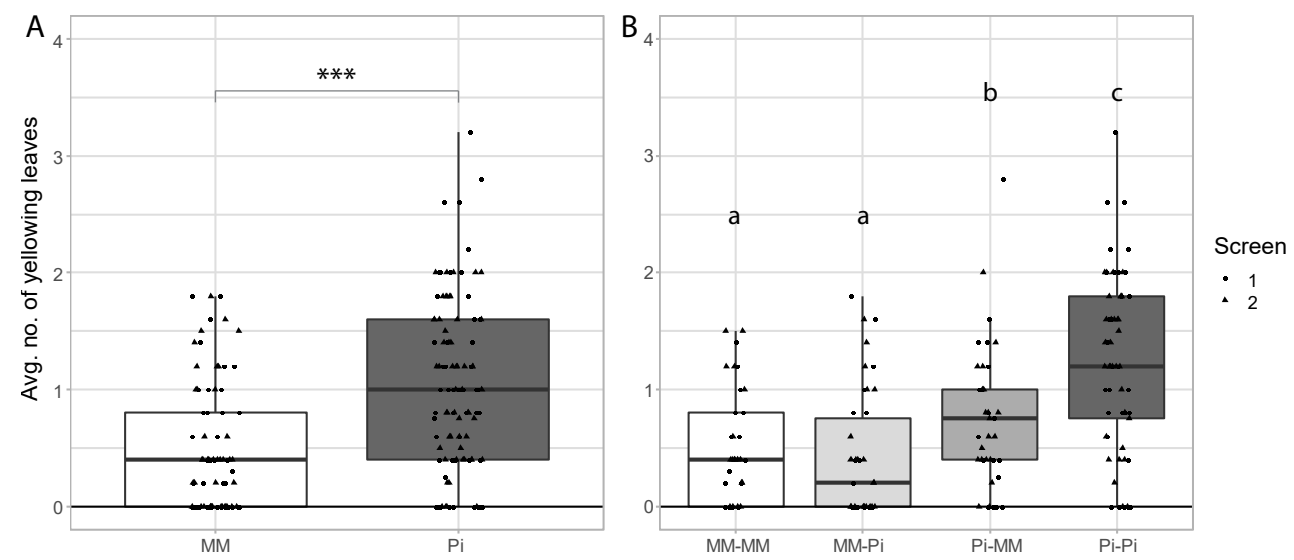

FIGURE 8 | (A) Effects of the QTL on cho3 on the number of yellowing leaves of V. dahliae-inoculated RILs (y-axis). "MM" alleles represent the allele from Moneymaker, "Pi" represents the alleles from VG-3. Asterisks indicate significant differences $(\mathrm{p}<0.01)$, determined with a Welch's t-test. (B) Effects of the QTL on cho3 and 5 on the yellowing phenotype (y-axis). Labels on the $\mathrm{x}$-axis represent the allele combinations of the QTL on cho3 and ch05, respectively. Different letter labels indicate significant differences according to a Welch's test $(p<0.05)$.

\section{QTL analysis of the resistance of S. pimpinellifolium VG-21}

\section{Randomizing RILs in pairs reduced differences in stunting between blocks}

In the Moneymaker x VG-3 RIL population, we observed a considerable degree of variability in stunting symptoms among plants of the same genotype. Therefore, we tried to improve the experimental design to reduce this variability in the Moneymaker x VG-21 RIL population. Given that we already determined theoptimal approach for both inoculation and phenotyping (chapter 2 of this thesis), we tested whether we could improve the randomization design of the experiment. Rather than randomly distributing each plant across the greenhouse compartment, we randomized the RILs across six experimental blocks in pairs of mocktreated and inoculated plants for the Moneymaker x VG-21 RIL population. In this manner, a mock-treated plant was always located immediately adjacent to a $V$. dahliae-inoculated plant of the same genotype. Furthermore, this enabled the calculation of stunting within a pair of a mock-inoculated and a V. dahliae-inoculated plant in the same block, which was not possible with the Moneymaker x VG-3 RIL population. Significant differences in the canopy area of mock-inoculated plants were observed between the blocks in both experiments (Fig. 9A-C), demonstrating that local environmental differences affected the growth of the plants. However, no differences in stunting of $V$. dahliae-inoculated plants were observed between the tables (Fig. 9 B-D), suggesting that the paired randomization design reduced the variability in stunting between blocks. 


\section{No QTLs could be identified for the quantitative resistance of VG-21}

In the Moneymaker x VG-21 RIL population, we only scored the stunting of V. dahliaeinoculated plants since it was impossible to reliably score $V$. dahliae-induced yellowing of the leaves due to symptoms like yellowing, necrosis or intumescence on the leaves of mock-inoculated plants. Similar to the Moneymaker x VG-3 RIL-population, the V. dahliae stunting symptom segregated quantitatively in the Moneymaker x VG-21 population (Fig. 10). In the two experiments, Moneymaker grouped with $38.2 \%$ and $43.6 \%$ stunting among the RILs with the highest degree of stunting, and VG-21 grouped with $7.9 \%$ stunting and $16.7 \%$ stunting among the RILs with the lowest degree of stunting. The F1 displayed an intermediate level of stunting, of 14.3 and $15.8 \%$ stunting, respectively

Similar as for the Moneymaker x VG-3 RIL population, the reproducibility of the symptoms was low. Several RILs displayed large differences in symptoms between the two experiments. Removal of these RILs (Fig. S7) from the dataset resulted in an increase of the reproducibility of $R^{2}=0.08$ to $R^{2}=0.17$ (Table S1). For this RIL population we did not find any evidence that variation in canopy area of mock-inoculated plants affected the degree of stunting (Fig. S8). We therefore did not include canopy area of mock-inoculated plants as a covariate in our analysis. No QTLs for stunting of $V$. dahliae-inoculated plants could be identified in this population, neither based on the individual screens, nor on the phenotypes of both screens combined. The estimated heritability of the stunting of $V$. dahliae-inoculated plants in this population was $H^{2}=0.10$ (Table S1), indicating that most of the variance in stunting was based on non-genetic factors.

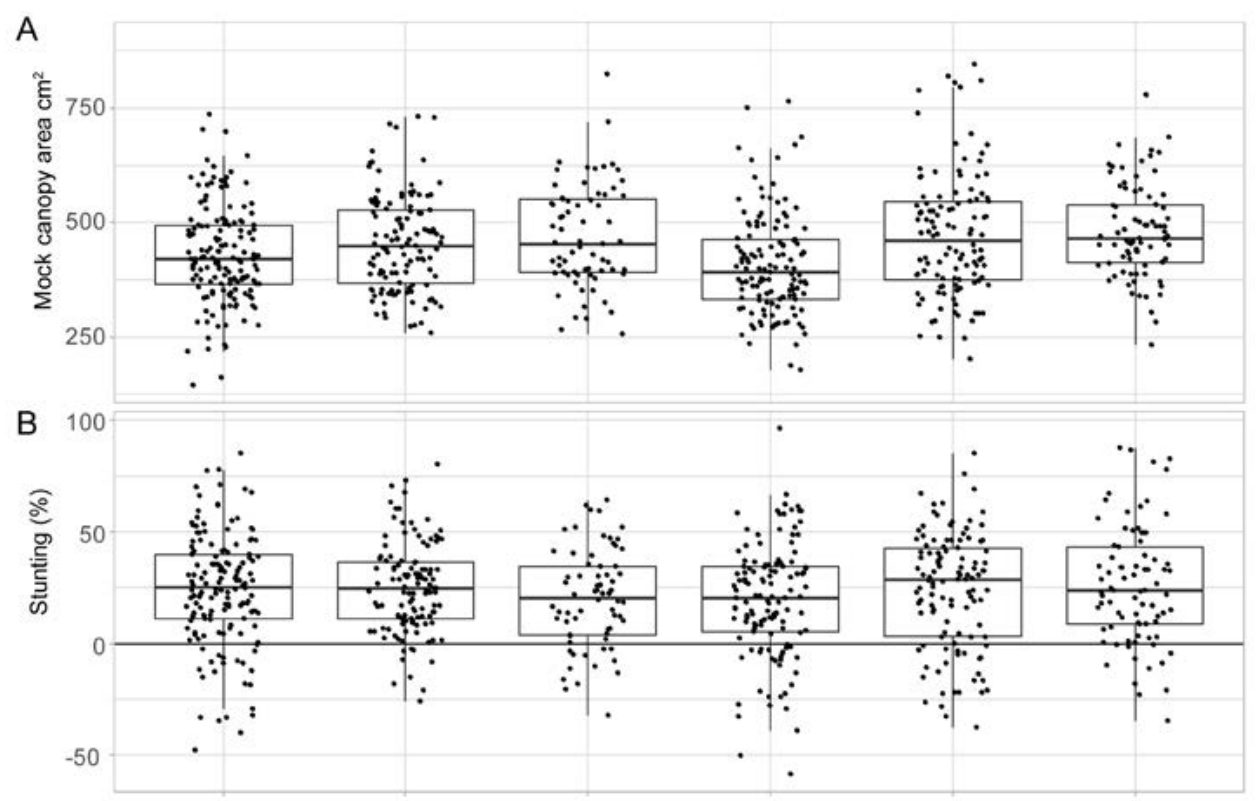




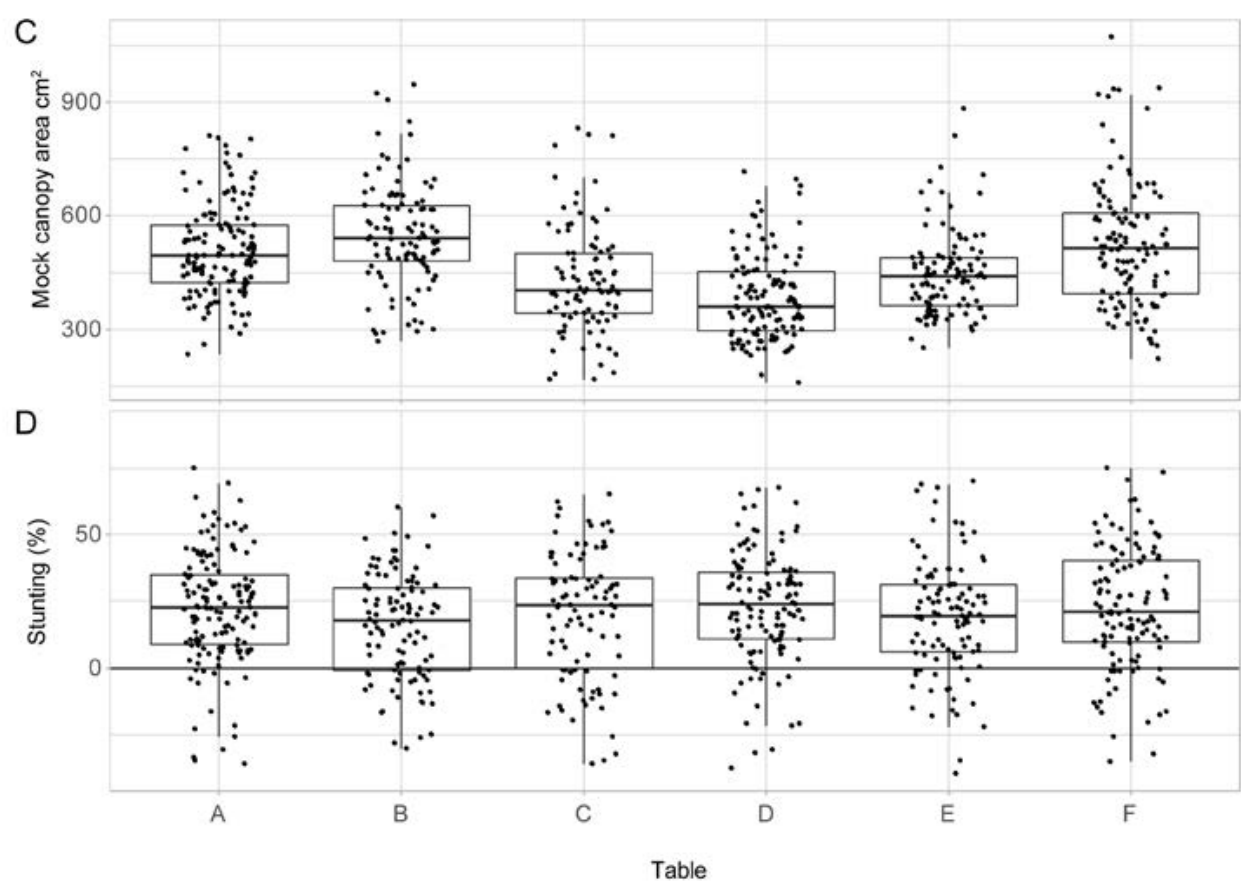

FIGURE 9 | Distribution of canopy area of mock-inoculated RILs of the S. lycopersicum Moneymaker x S. pimpinellifolium VG-3 population and the stunting of V. dahliae-inoculated RILS in the first (A-B) and second (C-D) RIL experiment. The X-axis illustrates the six experimental blocks in the greenhouse. Phenotypes were measured at 24-25 dpi. Different letter labels indicate significant differences as determined by a one-way ANOVA, followed by a Tukey's LSD test $(\mathrm{p}=0.05)$. 

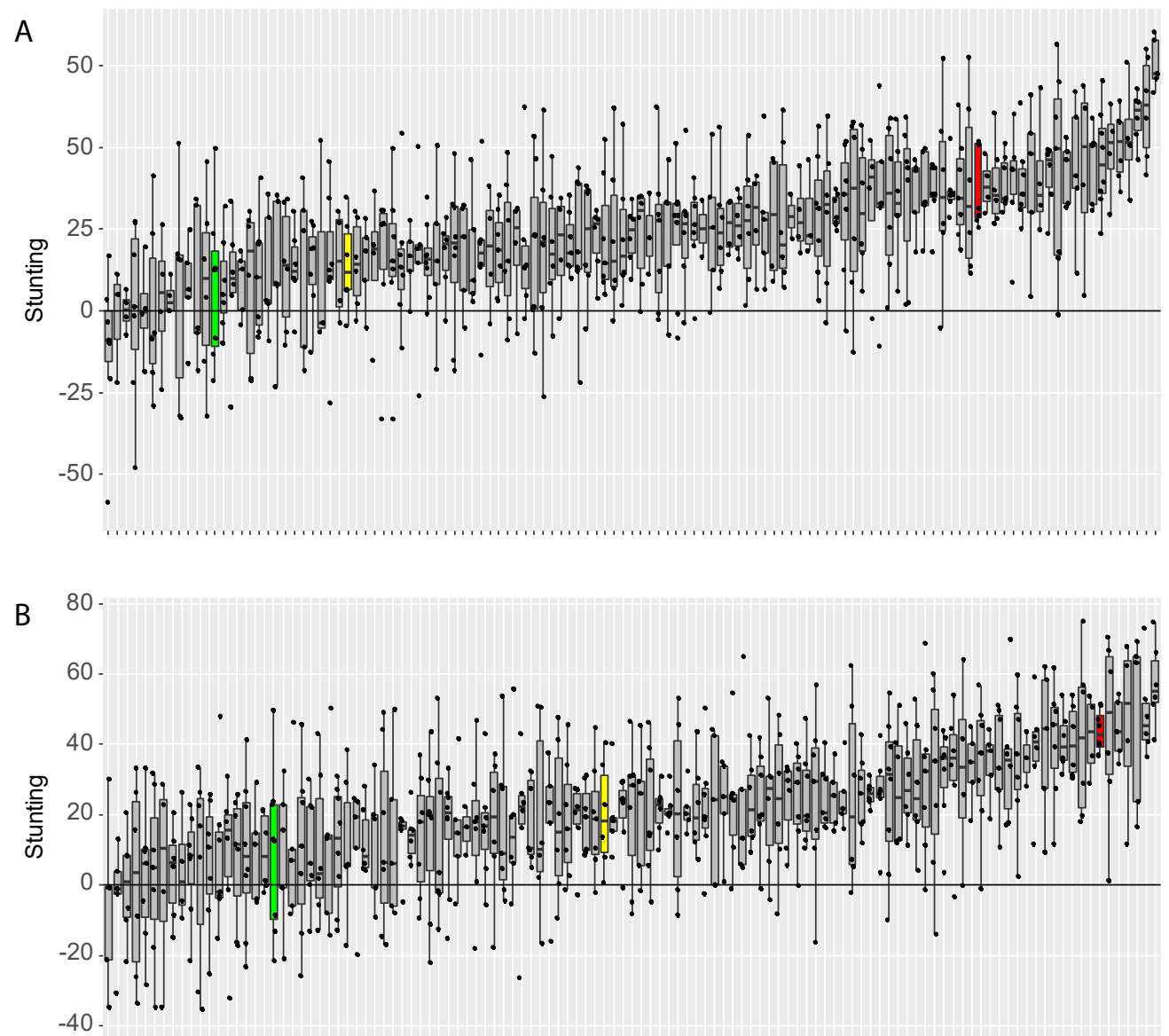

FIGURE 10 | Stunting of Moneymaker (red), VG-21 (green), the F1 (yellow), and the RILs (grey) at 24-25 days postinoculation with $V$. dahliae DVDS29. (A) represents the first and (B) represents the second experiment. Stunting reflects the relative reduction in canopy area of $V$. dahliae-inoculated plants relative to the canopy area of mockinoculated plants of the same genotype. 


\section{Discussion}

Although multiple findings of qualitative resistance against $V$. dahliae have been reported for tomato (Schaible et al. 1951; Usami et al. 2017), mapping studies in other crops typically report multiple QTLs for quantitative resistance against V. dahliae (Rygulla et al. 2008; Antanaviciute et al. 2015; Bolek et al. 2005; Wang et al. 2008; Zhao et al. 2014; Toppino and Barchi 2016). In this chapter, we studied the genetics of the $V$. dahliae resistance of two $S$. pimpinellifolium accessions, based on two Verticillium wilt-related symptoms.

In chapter 3, we demonstrated that S. pimpinellifolium VG-3 and VG-21 displayed reduced stunting symptoms and $V$. dahliae colonization compared to the susceptible control Moneymaker when challenged with a diverse set of $V$. dahliae strains, which demonstrates that these accessions possess resistance against $V$. dahliae. Unfortunately, based on the stunting symptoms, no QTLs could be identified for the resistance of these accessions. Several factors may have hampered the QTL analysis. First of all, the segregation of the stunting symptoms in both populations indicates that VG-3 and VG-21 possess quantitative resistance against $V$. dahliae, likely based on multiple genes. When a trait is quantitative and based on multiple genes, a mapping population of many hundreds of genotypes may be required to reliably identify QTLs (Li et al. 2010; Vales et al. 2005). Given that the QTL analyses with the Moneymaker x VG-3 and Moneymaker x VG-21 populations were performed with only 88 and 103 RILs, respectively, the power of our QTL analysis may have been too weak to accurately detect the QTLs. Secondly, the low estimated heritability of the stunting symptom in both populations suggests that a large proportion of the variation in the stunting symptom was caused by non-genetic factors. This may thus have further hampered our ability to detect QTLs.

Interestingly, the VG-3 RIL population not only segregated for stunting, but also for yellowing of leaves of $V$. dahliae-inoculated plants. Based on this yellowing symptom, several QTLs could be identified in the VG-3 population. It may thus be tempting to conclude that the yellowing symptoms may therefore be a more reliable $V$. dahliae symptom for QTL mapping. Indeed, although the estimated heritability of both phenotypes was similar, the reproducibility of the yellowing symptoms in the S. lycopersicum Moneymaker x VG-3 RIL population was higher than that of the stunting symptom, with correlation coefficients of $R^{2}=0.45 v s R^{2}=0.22$, respectively. However, the higher reproducibility of the yellowing symptoms is not the only explanation why we could not detect the same QTLs with both Verticillium wilt symptoms. Firstly, the stunting and yellowing symptoms segregated differently in the Moneymaker $\mathrm{x}$ VG-3 RIL population. Secondly, no meaningful correlation $\left(R^{2}=0.02\right)$ was found between stunting and yellowing of $V$. dahliae-inoculated plants (Fig S4). This suggests that different genetic mechanisms underly the segregation for stunting and the segregation for yellowing in $V$. dahliae-inoculated plants. Furthermore, although we showed in chapter 3 that VG-3 is more resistant to $V$. dahliae DVD3 than Moneymaker, we show in this chapter that VG-3 
plants did not display less yellowing of leaves than Moneymaker plants when challenged with $V$. dahliae DVD3. This suggests that a reduction of the yellowing symptoms may not be associated with resistance, but with tolerance to $V$. dahliae.

Even though QTLs were detected for the number of yellowing leaves in V. dahliae-inoculated plants, these QTLs did not fully explain the segregation of the yellowing symptoms in the RIL population. Whereas for the stunting, most RILs displayed an intermediate phenotype between the level of both parents, many of the RILs developed a higher degree of yellowing symptoms than both parents. These non-parental phenotypes in the RILs are often referred to as transgressive segregation. Furthermore, as the F1 also displayed more yellowing symptoms than both parents, it appears that the yellowing of leaves is also more severe in heterozygous plants. Interestingly, we also observed that Fi plants were larger than both parents (Fig. S5), indicating heterosis in these interspecific F1 hybrids. Heterosis is often the result of novel, non-parental combinations of alleles in the heterozygous F1, either by complementing deleterious alleles or synergistic interactions of the parental alleles. Because heterosis is based on heterozygous allele combinations, it is usually limited to the F1 and disappears in later generations because of increasing homozygosity (Baack and Rieseberg 2007). In contrast to heterosis, transgressive segregation is more trans-generationally stable because it is not caused by heterozygous allele combinations (Shivaprasad et al. 2012).

Over recent years, more light has been shed on the molecular mechanisms behind heterosis and transgressive segregation (de los Reyes 2019). During hybridization, changes in the transcriptome or epigenome can occur, which may result in non-parental phenotypes in the offspring. Several recent studies reported that small RNA molecules might play an important role in causing heterosis in F1 plants in different plant species (Crisp et al. 2019; Zhang et al. 2014a; Chen et al. 2010; Groszmann et al. 2011). Although the role of sRNAs in the expression of non-parental phenotypes in Fi tomato plants has not yet been extensively studied, their role in transgressive segregation has been demonstrated. For example, Shivaprasad et al. demonstrated that in an interspecific tomato cross, small-RNA molecules can accumulate in the F2 progeny and introgression lines which causes changes in gene expression, resulting in transgressive phenotypes (Shivaprasad et al. 2012). Currently, we have no evidence that small RNAs cause the transgressive phenotypes in the Moneymaker $x$ VG-3 population. However, it is known that small-RNAs can play an important role in plant-pathogen interactions. Several small-RNAs have been found to be involved in the regulation of defence-related genes, either by the enhancing or suppression of immune responses (Rose et al. 2019; Tang and Chu 2017). For example, in potato, overexpression of two miRNAs has been shown to result in increased sensitivity to V. dahliae (Yang et al. 2015). Furthermore, cross-kingdom RNA-based gene silencing is likely a common phenomenon in plant-microbe interactions and occurs in both directions (Wang et al. 2016; Chaloner et al. 2016; Weiberg et al. 2015). It may thus be worth to investigate the role of transcriptional changes and small RNAs in the transgressive phenotypes of the Moneymaker $\mathrm{x}$ VG-3 
RIL population. Given that transgressive segregation often occurs in both directions, it is a possibility that several of the RILs possess a degree of resistance or tolerance that is superior to that of both parents. Indeed, several RILs displayed no yellowing symptoms in both experiments, whereas both parents always displayed some yellowing of leaves when challenged with $V$. dahliae DVD3. However, given that even though Moneymaker is more susceptible to V. dahliae DVD3 than VG-3 (chapter 3), Moneymaker plants did not display more severe yellowing symptoms than VG-3 plants, it is unclear how yellowing symptoms in this RIL population relate to $V$. dahliae resistance. It may therefore be interesting to compare the level of $V$. dahliae colonization in RILs which displayed no yellowing symptoms with the colonization in the parents and RILs with the most severe yellowing symptoms. Furthermore, it may be worth to identify the loci which are differentially expressed between RILs with no yellowing symptoms and severe yellowing symptoms. If such differences in yellowing symptoms among RILs are indeed based on gene regulatory changes, an expression-QTL (eQTL) analysis (Michaelson et al. 2009) may help to unravel the loci that cause these regulatory changes. Although the identification of these eQTL and differentially expressed genes will not likely explain the resistance of VG-3, they may help to shed more light on the mechanisms that cause yellowing symptoms in $V$. dahliae-inoculated plants.

In the Moneymaker x VG-21 RIL population, several mock-inoculated RILs developed yellowing, necrosis and intumescence on the leaves. This made a reliable scoring of the number of yellowing leaves impossible. Because several RILs developed severe intumescence symptoms, this may also have caused more variation in the $V$. dahliae-related stunting symptom, resulting in a very low reproducibility and heritability of the phenotype. Given that also with the higher estimated heritability and reproducibility of the stunting symptom in the Moneymaker x VG-3 RIL population no QTLs were found, it is not surprising that also in the Moneymaker x VG-21 RIL population no QTLs were detected. The low estimated heritability in both populations suggests that a large proportion of the variance in stunting in both populations is based on non-genetic factors. This is likely the result of the large variation in stunting among RILs of the same genotype. Although we improved our methodology for the inoculation, phenotyping and randomization, this environmentally determined variation persisted. Partially, this may be because stunting is an indirect measure of $V$. dahliae resistance. After all, many other environmental factors which affect plant growth may also affect the stunting. Similarly, other genetic or environmental factors may affect the yellowing of leaves, as is illustrated by the yellowing of mock-inoculated plants in both populations.

Although methods exist to estimate the colonisation of $V$. dahliae in plants (Lievens et al. 2006), these methods are too labour-intensive for large-scale screenings of RIL populations. Therefore, in order to succeed in the analysis of QTLs for the resistance of VG-3 and VG-21, the current experimental design should be further improved. First, given the likely polygenic nature of the resistance, expanding the RIL population may help with 
the identification of QTLs. Secondly, increasing the number of genotypes per RIL will result in a more accurate estimate of its phenotype, which will thereby improve the QTL analysis. However, this will result in experiments consisting of many thousands of plants. Given the highly quantitative nature of the resistance, the usability of the resistance of VG-3 and VG-21 in breeding programmes is doubtful. Alternatively, if the Moneymaker x VG-21 RIL-population would be expanded, RILs which develop severe intumescence, yellowing or necrosis on the leaves can be excluded from the analysis. An exclusion of these RILs would allow the scoring of yellowing of leaves in V. dahliae-inoculated plants. Given the striking different segregation patterns of the stunting and yellowing symptoms in the Moneymaker $x$ VG-3 RIL-population, it would be valuable to test whether a similar phenomenon can be observed in the Moneymaker x VG-21 RIL population. Although these transgressive segregation patterns would likely not explain the resistance of VG-3 and VG-21, unravelling their genetics in both populations may further help to gain novel insights in the mechanisms behind the development of yellowing leaves in $V$. dahliae-inoculated plants. 


\section{Supplemental material}

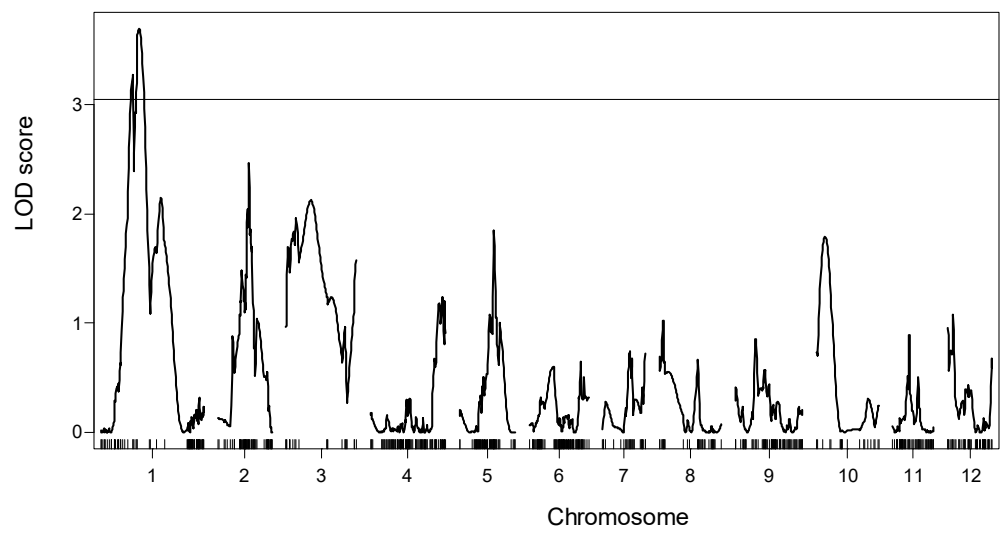

SUPPLEMENTAL FIGURE 1 | Results from simple interval mapping for mock canopy area in the S. lycopersicum Moneymaker x S. pimpinellifolium VG-3 RIL population. Horizontal line indicates the significance threshold ( $\mathrm{p}<$ 0.05), which was determined with a permutation test with 1000 permutations.

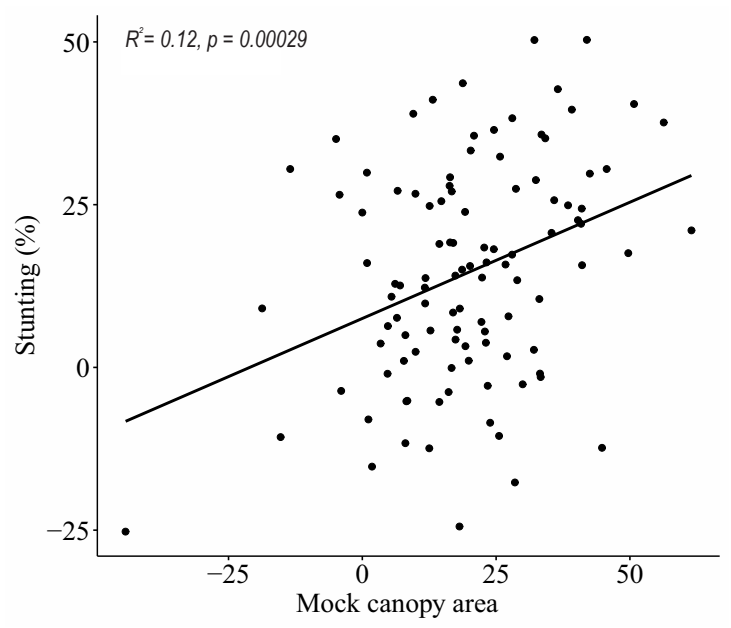

SUPPLEMENTAL FIGURE 2 | Pearson correlation analysis between the canopy area of mock-inoculated plants and the stunting V. dahliae-inoculated plants of the S. lycopersicum Moneymaker x S. pimpinellifolium VG-3 RIL population. 


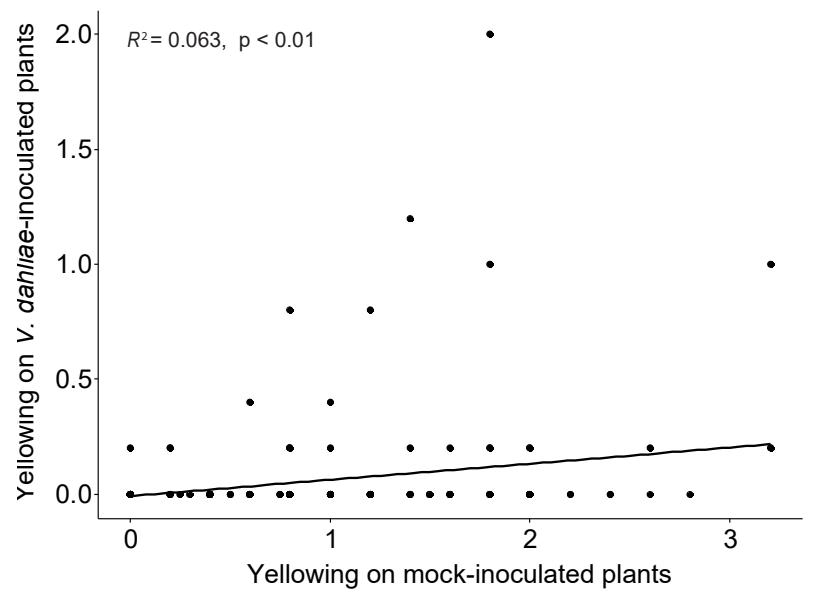

SUPPLEMENTAL FIGURE 3 | Spearman Correlation analysis between the number of yellowing leaves of mockinoculated and $V$. dahliae-inoculated plants in the Moneymaker VG-3 RIL population.

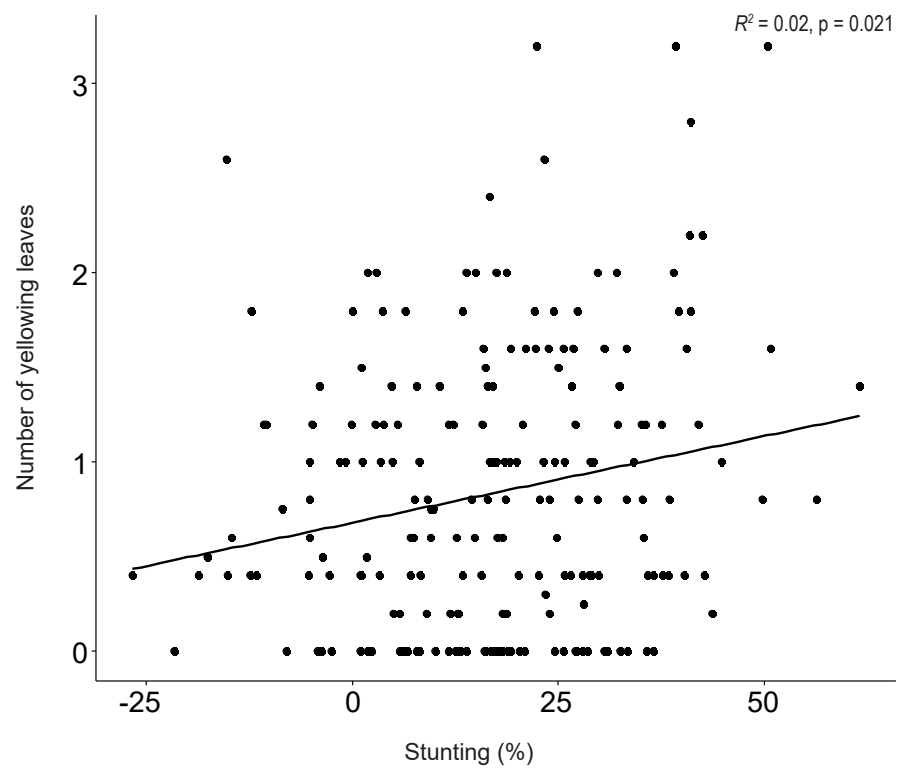

SUPPLEMENTAL FICURE 4 | Spearman Correlation analysis between the number of yellowing leaves and stunting of $V$. dahliae-inoculated plants in the Moneymaker x VG-3 RIL population. 


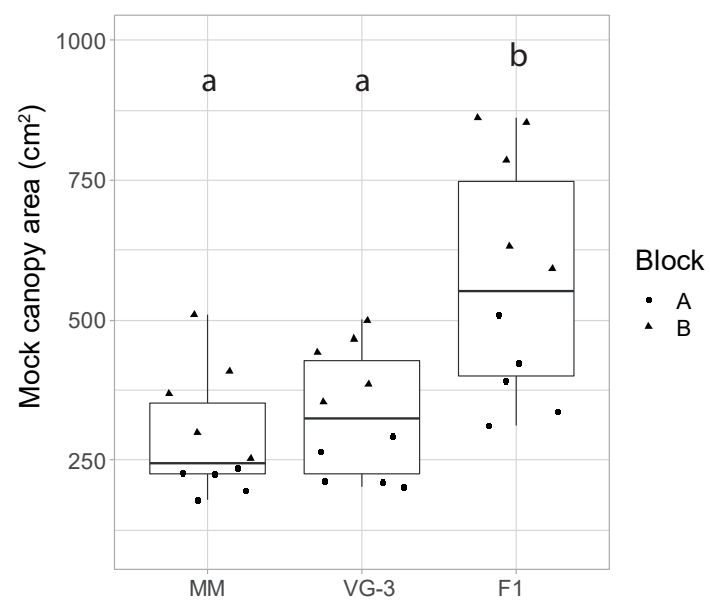

SUPPLEMENTAL FIGURE 5 | Comparison of the canopy area $\left(\mathrm{cm}^{2}\right)$ of mock-inoculated plants of the $S$. lycopersicum Moneymaker parent (MM), the S. pimpinellifolium VG-3 parent and the F1. Different letter labels indicate significant differences as determined by a one-way ANOVA followed by a fishers LSD test $(\mathrm{p}<0.01)$.

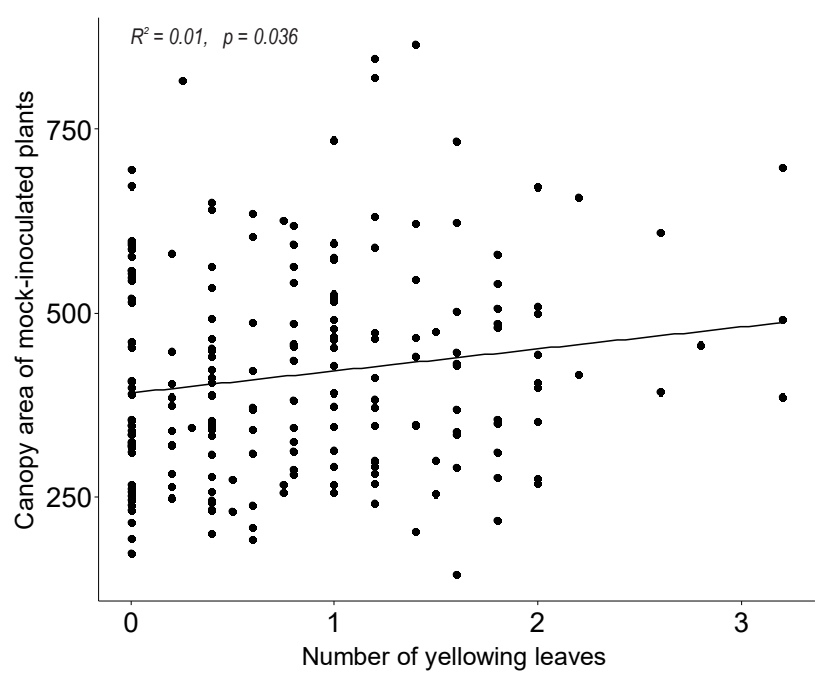

SUPPLEMENTAL FIGURE 6 | Kendall rank correlation between the average canopy area of mock-inoculated RILs and the average number of yellowing leaves of $V$. dahliae-inoculated RILs of the S. lycopersicum Moneymaker $\mathrm{x} S$. pimpinellifolium VG-3 population. 


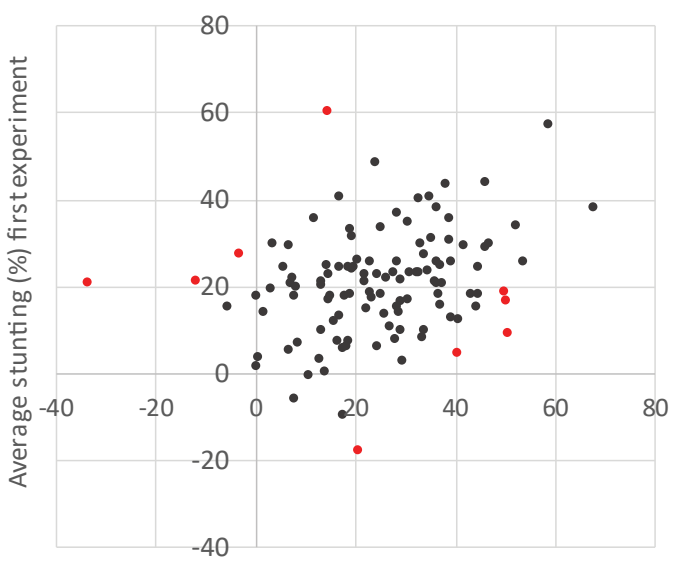

Average stunting (\%) second experiment

SUPPLEMENTARY FIGURE 7 | Comparison of the average stunting of the S. lycopersicum Moneymaker x $S$. pimpinellifolium VG-21 RILs in the first screen ( $\mathrm{x}$-axis) and the second screen (y-axis). Red dots show the RILs with the least reproducible phenotypes.

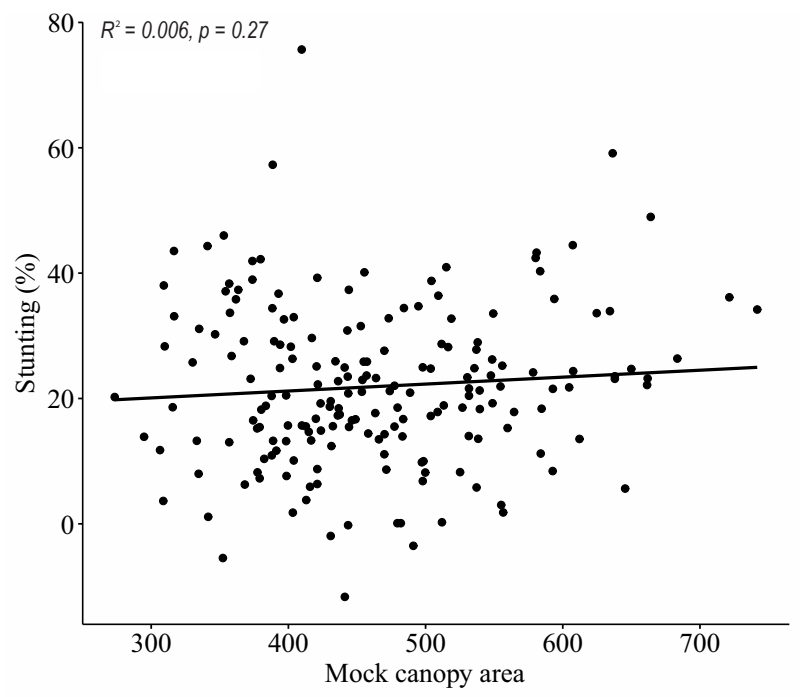

SUPPLEMENTAL FIGURE 8|Pearson correlation analysis between the Canopy area of non-inoculated plants and the level of stunting of inoculated plants of the S. lycopersicum Moneymaker x S. pimpinellifolium VG-21 RIL population. 
SUPPLEMENTARY TABLE 1 | Heritability estimates and correlation coefficients.

\begin{tabular}{|c|c|c|c|c|}
\hline $\begin{array}{c}\text { Heritability }\left(H^{2}\right) \text { and } \\
\text { correlation coefficient } \\
\left(R^{2}\right)\end{array}$ & $H^{2}$ experiment 1 & $H^{2}$ experiment 2 & $\begin{array}{c}H^{2} \text { experiment } 1+2 \\
\text { combined }\end{array}$ & $\begin{array}{c}R^{2} \text { between } \\
\text { experiment } 1 \text { \& } 2\end{array}$ \\
\hline All RILs & 0.14 & 0.08 & 0.06 & 0.08 \\
\hline $\begin{array}{l}\text { Only RILs with the most } \\
\text { consistent phenotype }\end{array}$ & 0.09 & 0.081 & 0.10 & 0.17 \\
\hline
\end{tabular}

Estimates of the broad-sense heritability of the stunting phenotype in the S. lycopersicum Moneymaker $\mathrm{x} S$. pimpinellifolium VG-21 RIL population, with either all RILs or only the RILs with a reliable phenotype. Heritability was estimated using a one-way ANOVA.
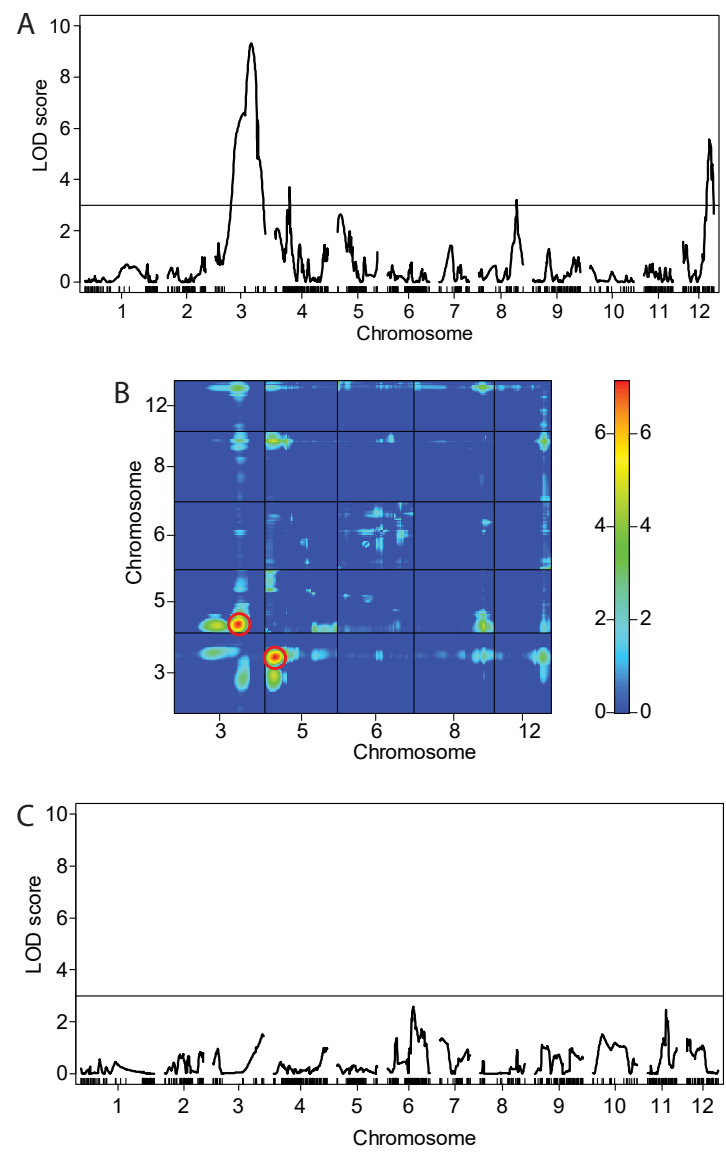

SUPPLEMENTARY FIGURE 9 | LOD-profiles from the interval mapping $(A, C)$ and $2 D$ two-QTL (B) analyses based on yellowing $V$. dahliae symptoms of inoculated plants (A, B) and mock-treated plants (C) of the S. lycopersicum Moneymaker x S. pimpinellifolium VG-3 RIL population. (A, C) the horizontal line represents the significance threshold ( $\mathrm{p}<0.05$ ), determined with a permutation test (1000 permutations). (B) Red circles highlight positions with significant LOD scores for interactions between two QTLs $(p<0.05)$. The significance threshold was determined using a two-dimensional permutation test with 400 permutations. LOD scores of (B) indicate the likelihood of a pair of two QTLs over the likelihood of a single QTL, allowing for epistatic interactions. 


\title{
Chapter 5
}

\section{At least three QTLs are associated with the resistance of Solanum cheesmanii VG-20 to a race 2 strain of Verticillium dahliae}

\author{
Jasper P. Vermeulen ${ }^{1,2}$, \\ Bart P.H.J. Thomma ${ }^{1,3}$, \\ Yuling $\mathrm{Bai}^{2}$, \\ Henk J.Schouten ${ }^{2}$
}

'Laboratory of Phytopathology, Wageningen University \& Research, 6708 PB Wageningen, The Netherlands. ${ }^{2}$ Laboratory of Plant Breeding, Wageningen University \& Research, 6708 PB Wageningen, The Netherlands.

${ }^{3}$ Cluster of Excellence on Plant Sciences (CEPLAS), University of Cologne, Botanical Institute, Cologne, Cermany. 


\section{Abstract}

Vascular wilts caused by Verticillium dahliae are among the most destructive soil-borne fungal diseases, which can result in over $50 \%$ yield losses in some crops. Because most fungicides are ineffective once $V$. dahliae has entered the plant, and because the fungus produces resting structures that can stay viable for many years, Verticillium wilt disease is difficult to control. Therefore, the preferred method to control Verticillium wilt has been to identify and employ host resistance. Up to this date, only one Verticillium wilt resistance gene, Ve1, has been identified and cloned in tomato. Soon after the first tomato crops with Ve1 resistance were introduced in the 1950s, race 2 strains that overcame this resistance emerged in the US and in Europe. Although several accessions with resistance against race 2 strains of $V$. dahliae have been reported, the corresponding genes or QTLs have not yet been identified. We previously identified two $S$. pimpinellifolium accessions and one $S$. cheesmanii accession that exhibit resistance against $V$. dahliae race 2 strains. Previous mapping studies failed to identify QTLs for the resistance of both S. pimpinellifolium VG-3 and VG-21 but led to the identification of QTLs for yellowing symptoms contributed by the susceptible parent $S$. lycopersicum Moneymaker. In this study, based on stunting symptoms, we present evidence for two QTLs for V. dahliae race 2 resistance on chromosome 1 and 7 of VG-20. Secondly, also two QTLs from Moneymaker associated with reduced stunting symptoms were detected, of which one overlaps with the QTL on chromosome 3 we previously identified in the Moneymaker x VG-3 RIL population. 


\section{Introduction}

Verticillium wilt is a destructive vascular disease, causing severe damage to many crop species (Tabaeizadeh et al. 1999; Zeise and Von Tiedemann 2002; Klosterman et al. 2009; Fradin and Thomma 2006). Although Verticillium species possess the machinery for sexual reproduction, they are thought to have a predominantly asexual lifestyle (Short et al. 2014; De Jonge et al. 2013). The Verticillium genus presently consists of 10 species, of which five are known as plant pathogens, while the other five species are thought to mainly have saprophytic lifestyles (Inderbitzin et al. 2011). Of the five pathogenic species, Verticillium dahliae is economically the most important, infecting hundreds of plant species worldwide (Klosterman et al. 2009; Fradin and Thomma 2006). V. dahliae is particularly problematic because it produces resting structures called microsclerotia, which can stay viable in the soil for many years (Wilhelm 1955). When exudates from nearby host roots trigger microsclerotia to germinate, hyphae penetrate the root epidermis and grow towards the root cortex to enter the xylem vessels. Once inside the xylem, the fungus produces conidiospores that spread throughout the plant (Fradin and Thomma 2006; Klosterman et al. 2009). Although the type and degree of Verticillium wilt symptoms depend strongly on environmental conditions and host species, they generally consist of stunted growth, wilting, yellowing and chlorosis of tissues (Pegg and Brady 2002; Fradin and Thomma 2006; Klosterman et al. 2009). In susceptible plants, yield losses commonly range from 10 to $15 \%$, but losses over $50 \%$ have been reported for multiple economically valuable crops such as potato, cotton, strawberry and lettuce (Dean et al. 2014; Pegg and Brady 2002; Klosterman et al. 2009).

In the late $19^{\text {th }}$ and early $20^{\text {th }}$ century, Verticillium wilts first caught the attention of scientists after the pathogen began to cause severe damage in Europe and the US (van der Lek 1919; Rudolph 1931). Because vascular wilts such as Verticillium wilts reside inside the plant, few methods exist to control the disease once plants are infected. Due to its broad host range and longevity of its microsclerotia, crop rotation is often not effective to control Verticillium wilts. Some control methods exist to reduce the inoculum quantities in the soil, mostly by solarisation and fumigation. However, the first is only useful in warm and sunnier climates, and the latter has been severely restricted due to environmental regulations. The preferred strategy to control the disease, already since the first reports of Verticillium wilts in the early $20^{\text {th }}$ century (van der Lek 1919; Rudolph 1931), has therefore been the use of host resistance. Soon after the first Verticillium wilts were reported, multiple resistance sources were found (Sherbakoff 1949). In only one of these sources, a wild Peruvian tomato accession, a dominantly inherited locus called " $V e$ " was identified (Schaible et al. 1951). After several failed attempts to locate the Ve locus, it was finally mapped on chromosome 9 (Diwan et al. 1999; Zamir et al. 1993; Juvik et al. 1991; Kerr and Busch 1977, 1975). Further research then revealed that the Ve locus contains two receptor-like protein-encoding genes, Ve1 and Ve2, of which $V e 1$ is the causal resistance gene (Kawchuk et al. 2001; Fradin et al. 2009). Unfortunately, 
soon after the first Ve-resistant cultivars were released in 1953 (Deseret News and Telegram 1955), Verticillium strains were reported to cause disease on plants harbouring the Ver gene; first in the US (Alexander 1962; Robinson 1957) but soon also at multiple locations in Europe (Pegg and Dixon 1969; Cirulli 1969; Tjamos 1976). The Verticillium population was therefore split into two races based on the ability to cause disease on tomato cultivars carrying the Ver gene (Alexander 1962). Whereas race 1 strains are contained by Ve1 resistance, race 2 strains escape recognition and cause disease on plants that carry Ver. The increasing emergence of race 2 strains has led to a search for novel resistance sources. Although multiple additional race 2-resistant tomato accessions have been reported (Okie and Gardner 1982b, 1982a; Latterot 1984; Baergen et al. 1993a; Stamova 2005; Yadeta 2012; Klosterman et al. 2009), no QTLs or genes have been mapped for this resistance yet. However, a recent report describes resistance against race 2 of $V$. dahliae in the tomato rootstock cultivar Aibou (Usami et al. 2017), which is likely based on a dominant $R$ gene named "V2". Interestingly, while characterizing this resistance, strains of $V$. dahliae were identified that can overcome the V2-resistance in Aibou. Thus, V. dahliae can be divided into three races based on the resistance-breaking capabilities of $V$. dahliae strains. Strains which are controlled by both $V e 1$ and $V 2$ should be assigned to race 1 , whereas strains which have overcome the resistance of Ve1 or V2 should then be reassigned to race 2 and 3, respectively.

Previously, an extensive collection of wild tomato accessions has been screened at Wageningen University for resistance against race 2 strains of V. dahliae (Yadeta 2012). Several of these accessions displayed a reduction in $V$. dahliae-associated stunting symptoms (Yadeta 2012), and in chapter 3 of this thesis we confirmed the resistance in three of these accessions; Solanum pimpinellifolium VG-3, Solanum pimpinellifolium VG-21 and VG20. Of these three, especially VG-20 and VG-21 displayed a reduction in stunting symptoms and $V$. dahliae colonization upon inoculation with several race 1, 2 and 3 strains. For both accessions, Recombinant Inbred Lines (RIL) populations were developed to map QTLs for the race 2 resistance. Unfortunately, for the resistance of VG-3 and VG-21, no significant QTLS could be identified (chapter 4 of this thesis).

VG-20 was initially thought to belong to $S$. lycopersicum, but sequencing analysis revealed it to be derived from a cross between $S$. cheesmanii and $S$. lycopersicum. This means that although it is morphologically similar to $S$. lycopersicum, it also contains genetic material from S. cheesmanii. This made VG-20 an interesting candidate for QTL analysis, as the reduced morphological variation in a segregating population derived from a cross of VG-2O and the susceptible control S. lycopersicum Moneymaker may increase the power of genetic mapping analyses. In this study, we describe the screening of a RIL population derived from Moneymaker and VG-20 for resistance against $V$. dahliae. We found evidence for multiple QTLs associated with resistance against the $V$. dahliae race 2 strain DVDS29, both from VG20 and from Moneymaker. 


\section{Materials \& methods}

\section{Development of RIL-populations and genotyping}

To produce the RIL-population, S. cheesmanii VG-20 was crossed with S. lycopersicum Moneymaker with the latter as the mother plant. After selfing of F1 plants, seeds of the F2 population were further selfed by single seed descent until the F6 was reached. To genotype the RIL population, leaves were harvested from the F5 plants of which the F6 seeds were harvested. To select SNP markers for the genotyping of the RIL population, all markers from Sim et al. (2012) were tested on VG-20 and Moneymaker. Then, based on the tomato EXPMIMP 2009 genetic map (Ashrafi et al. 2009), the genetic positions of the markers were interpolated onto this map. Out of all 7,688 SolCap-SNPs, 288 SNP markers (Table S1, Sim et al. 2012) were selected for genotyping the RIL population that are distributed across the genome with intervals of on average $4 \mathrm{cM}$.

\section{Marker validation and construction of the genetic map}

Before constructing the genetic map, all markers were first visually inspected. Markers of which the genotyping resulted in no data or only heterozygous data for all genotypes were removed. Next, using the R-packages R/QTL and AsMAP (Taylor and Butler 2017), markers were validated by checking for (1) unusually skewed segregation of markers, (2) markers with $>10 \%$ of missing data, (3) identical markers on different physical positions, and (4) unusually high crossover rates between physically closely located markers. Markers which were considered as unreliable based on these criteria, 25 in total, were removed. Using the "MSTmap" function of the R package ASMap (Taylor and Butler 2017), linkage groups were constructed for each chromosome based on the 263 remaining markers (Table S1) with a p-value of $10^{-4}$ with the options "anchor" and "byChr" enabled. The physical order and genetic order were evaluated using the "ripple" function of R/QTL. If the genetic order and physical order of markers were not in agreement, the likelihood of both marker orders was estimated using the "ripple" function of the package R/QTL version 1.45-6 (Broman et al. 2003). If the genetic order and physical order of the markers was equally likely, we favoured the physical order of the markers.

\section{Preparation of inoculum and inoculation procedures}

Five petri dishes with Potato Dextrose Agar were inoculated with V. dahliae strain DVDS29 from a $-80^{\circ} \mathrm{C}$ glycerol stock. Plates were grown at $24^{\circ} \mathrm{C}$ for at least 5 days, after which the agar was cut into blocks of $\sim 1 \mathrm{~cm}^{2}$. These blocks were then transferred to a freshly made potato sucrose broth that was produced by boiling 400 grams of pre-peeled potato dices in $1 \mathrm{~L}$ of water for 15 minutes, after which the water was filtered through cheesecloth. Per L of broth, $\mathrm{IL}$ of water and $40 \mathrm{~g}$ of sucrose was added, followed by autoclaving for 30 minutes at $120^{\circ} \mathrm{C}$. After inoculating the broth with the inoculated agar blocks, it was kept in an incubator 
for at least 5 days at $24^{\circ} \mathrm{C}$ at $100 \mathrm{rpm}$. Using Mira cloth, mycelium was filtered out of the broth. Subsequently, the inoculated broth was centrifuged at 3,500 rpm for 30 minutes and the supernatant was removed. Next, the conidiospores were diluted in tap water to a concentration of $10^{7}$ conidiospores/mL. For inoculation, 12-day-old seedlings were carefully uprooted and the roots were rinsed in tap water. Mock-inoculated plants were root- dipped in water for 10 minutes, whereas other plants were root-dipped for 10 minutes in the inoculum. Of each genotype, on average, 12-14 plants were used of which half were inoculated with $V$. dahliae and the other half were mock-inoculated. After inoculation, seedlings were potted into trays of small cups containing sowing soil. From these trays, the cups were transferred to $14 \mathrm{~cm}$ pots according to the randomization scheme described below. Plants in block 1 and 2 were randomized one day after the inoculation, plants in block 2 and 3 two days after inoculation and the plants in block 5 and 6 three days after inoculation.

\section{Experimental design and randomization}

The replicate plants of each genotype were randomised across the greenhouse compartment in six blocks. The randomisation was performed in such a way that in each block, every genotype was represented by one replicate mock- and one replicate $V$. dahliae-inoculated plant immediately adjacent to each other. The remaining plants of each genotype were then randomly assigned to one of the blocks. All genotypes were furthermore randomised across the blocks in pairs of mock-inoculated and inoculated plants. (Fig. 1). By doing so, of each genotype, a mock-inoculated plant was located adjacent to a $V$. dahliae-inoculated plant of the same genotype (Fig. 1).
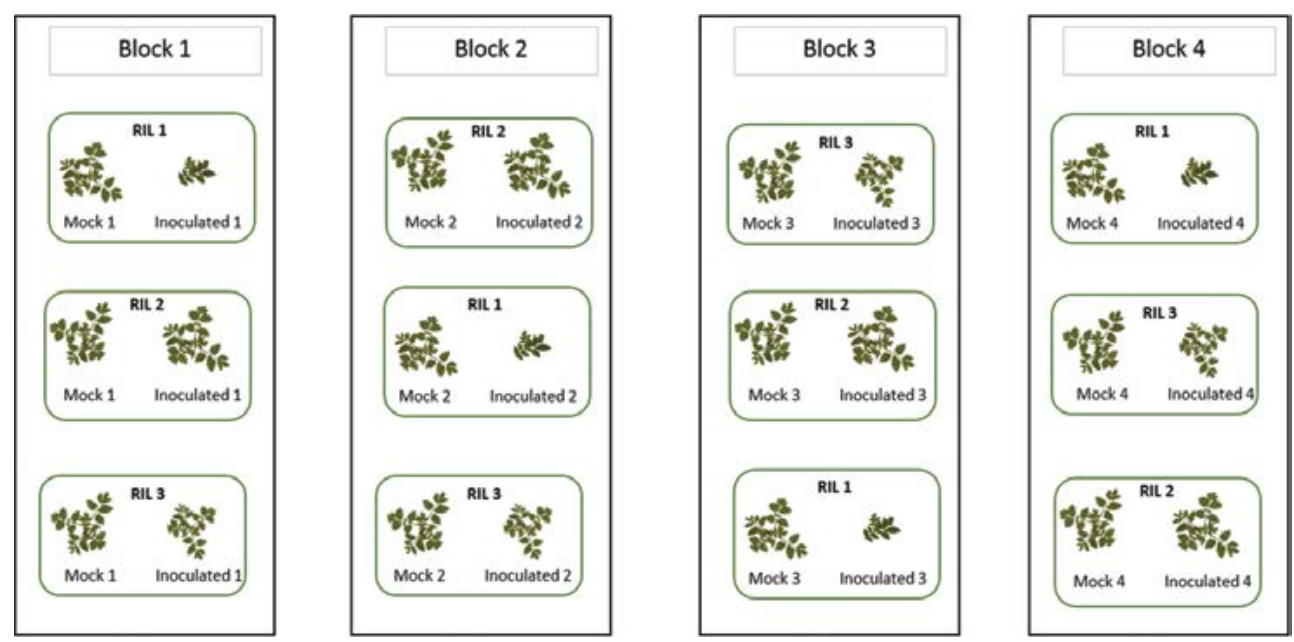

FIGURE 1 | Overview of the paired randomised block design that was used to position plants in the greenhouse. Plants were randomised across the greenhouse over six blocks in pairs of mock-inoculated and $V$. dahliae-inoculated plants such that a mock-inoculated and $V$. dahliae-inoculated plant of the same genotype were always adjacent to each other. 


\section{Phenotyping}

Blocks 1 to 3 were phenotyped at 24 dpi and blocks 4 to 6 at 25 dpi. The canopy of individual plants was photographed from the top using a Nikon D3200 DSLR camera that was fixed on a tripod. The canopy area of the plants was determined with ImageJ version 1.51f (Schneider et al. 2012). The stunting of $V$. dahliae-inoculated plants was calculated by comparing the canopy area of the $V$. dahliae-inoculated plant with the canopy area of mock-inoculated plant within each pair using the following formula:

$$
\text { Stunting }(\%)=\left(1-\frac{\text { Canopy area of inoculated plant of pair } x \text { of genotype } y}{\text { Canopy area of mock-treated plant } x \text { of genotype } y}\right) * 100
$$

To test for significant differences in stunting, a one-way ANOVA was performed for stunting. Outliers were detected based on the studentized residuals from the ANOVA analysis. Datapoints with studentized residuals below -2.5 or above 2.5 were considered as outliers and removed.

The broad-sense heritability was estimated from the output of a one-way ANOVA using the formula below, with $\sigma_{e}^{2}=M S_{\text {residual }}$ and $\sigma_{g}^{2}=\frac{M S_{\text {genotype }}-\sigma_{e}^{2}}{\text { average number of replicates per genotype }}$ Estimated broad sense heritability $\left(H^{2}\right)=\frac{\sigma_{g}^{2}}{\sigma_{g}^{2}+\sigma_{e}^{2}}$

\section{QTL analysis in $\mathbf{R}$}

The QTL analysis was performed with version 1.45-6 of the R package R/QTL (Broman et al. 2003). The QTL analysis consisted of three steps. First, using the function "scanone" an interval mapping analysis was performed using the "em" algorithm. A permutation test with 1000 permutations was then performed with the "scanone" function to determine the significance threshold ( $\mathrm{p}<0.05$ ). For the QTL analysis, two covariates were added. As the phenotypes from both experiments were included in the same analysis, one covariate was used to separate the phenotypes from both experiments. As a second covariate the average canopy area of mock-inoculated plants of each RIL was included. After the "scanone" analysis, the "scantwo" analysis was performed using the "em" algorithm. Also, in this case, a permutation test was performed. Due to the high amount of computational time required for this permutation test, the permutations were divided into ten batches using the "n.batch" option and were analysed on a Linux-based server cluster. All evidence for QTLs found with "scanone" and "scantwo" were combined into a multiple QTL model using the function "makeqtl", followed by "fitqtl", and refined with the "refineqtl" function. Using the function "stepwiseqtl", the most likely multiple QTL model was then selected. The significance threshold for this analysis was determined using the permutation test of "scantwo" with the "imp" algorithm. 


\section{Results}

\section{VG-20 possesses quantitative, dominantly inherited resistance against $\mathrm{V}$. dahliae race 2 strain DVDS29}

To study the genetics of the Verticillium wilt resistance of VG-20, we developed an F6 RILpopulation from a cross with the susceptible S. lycopersicum cultivar Moneymaker. Based on the results from chapter 3 , we selected the race 2 strain to which (1) the accession displayed both a reduction in stunting symptoms and a reduction in pathogen biomass and (2) that provided a clear contrast in stunting symptoms between the susceptible and resistant parent (chapter 3 of this thesis); For VG-20, this was V. dahliae strain DVDS29.

To phenotype the Verticillium wilt symptoms, we initially aimed to score the number of yellowing leaves as well as the stunting of $V$. dahliae-inoculated plants. However, as in many of the RILs the lower leaves became yellow without $V$. dahliae inoculation (Fig. S1), we decided to only measure the stunting of $V$. dahliae-inoculated plants. Typically, Verticillium wilt symptoms such as yellowing of leaves and stunting display a large degree of variation, which can make QTL mapping challenging. To increase the accuracy of phenotyping, we used on average 12 plants for each genotype, half of which were $V$. dahliae-inoculated while the other half were mock-inoculated. To furthermore attempt to reduce phenotypic variation, plants were randomized across six blocks in pairs of mock-inoculated and $V$. dahliae-inoculated plants. Unfortunately, significant differences were observed in the canopy area between the blocks in both experiments (Fig. S2 A, C). However, no differences in the degree of stunting, i.e. the canopy area ratios for pairs of mock-inoculated and $V$. dahliae-inoculated plants, were observed between the blocks (Fig. S2 B, D). This suggests that like in the VG-3 RIL population (chapter 4 of this thesis), the paired randomisation design likely reduced variability in stunting between the experimental blocks.

Moneymaker grouped among the most susceptible genotypes that displayed the highest degree of stunting in both phenotyping screens ( $45.8 \%$ and $47.45 \%$ ), while the F1 and VG-20 grouped among the resistant genotypes with the lowest degree of stunting $(9.18 \%, 16.5 \%$ stunting for VG-20, $0 \%$ and $9.5 \%$ stunting for F1) (Fig. 2), confirming the previously found resistance of VG-20. The low degree of stunting of the F1 in both screens suggests a dominant inheritance of the resistance. In the VG-20 population, we also observed a continuum of stunting levels between the most susceptible and the most resistant plants, suggesting that VG-20 possesses quantitative resistance against $V$. dahliae, most likely based on multiple genes.

In each screen, a considerable degree of variation in stunting was observed among $V$. dahliae-inoculated plants of the same genotype (Fig. 2). Furthermore, eight RILs (Fig. 3) showed large variation of stunting between the two experiments. This likely resulted in a 
rather low reproducibility of the symptoms between the two experiments with $R^{2}=0.10$. The eight RILs in particular displayed a difference in more than $25 \%$ stunting between the two experiments and removing these eight RILs improved the reproducibility to $R^{2}=$ 0.26. Therefore, these eight RILs were not included in the QTL analysis. Besides the low reproducibility, also the estimated broad-sense heritability was low, ranging between $H^{2}=0.21$ and $H^{2}=0.16$ (Table 1 ) for the first and second screen, respectively. This indicates that a large degree of the variation in stunting of $V$. dahliae-inoculated plants was due to non-genetic factors. Removal of the eight previously mentioned RILs did not considerably improve the estimated heritability.
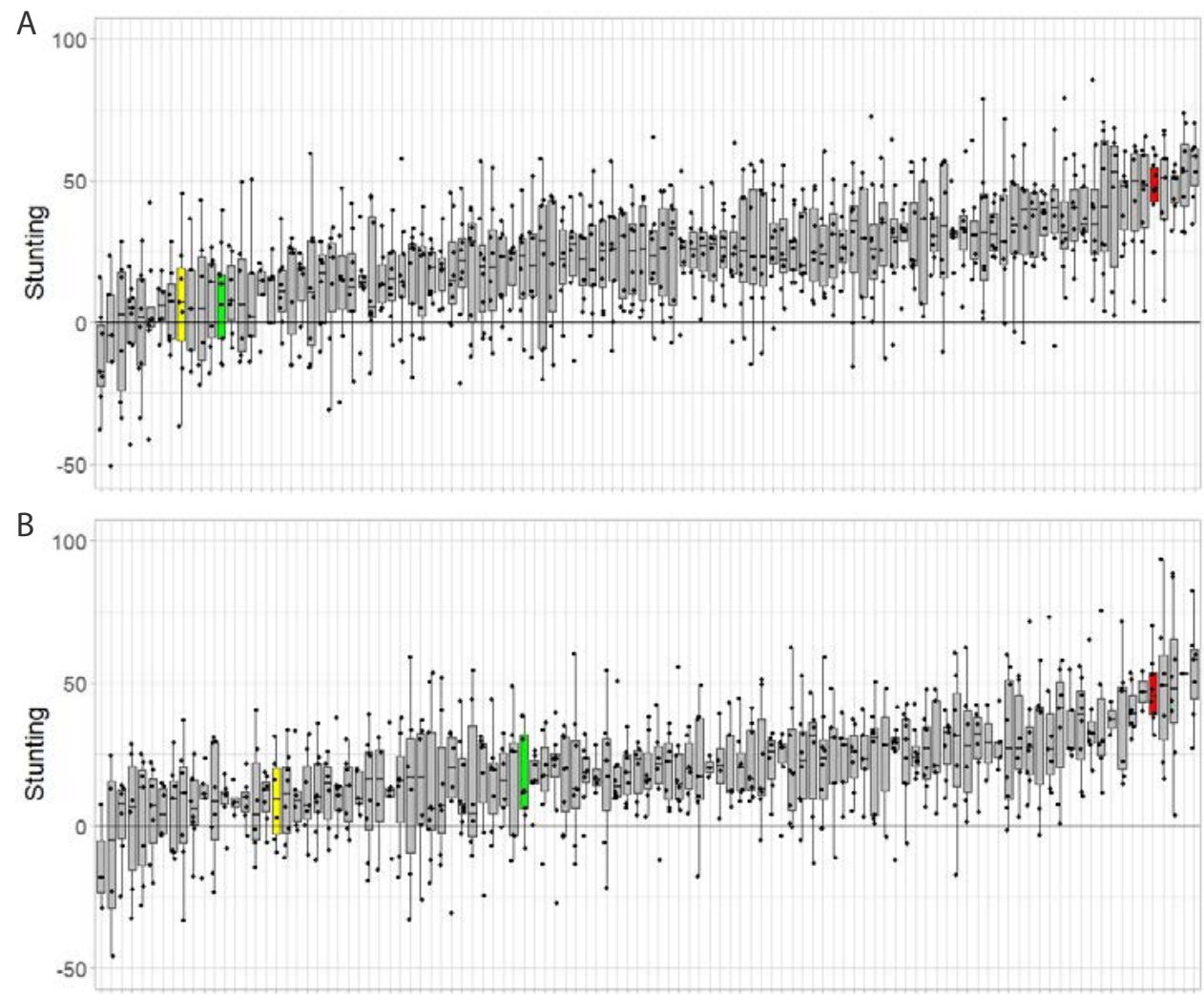

FICURE 2 | Stunting of V. dahliae-inoculated plants of the Moneymaker parent (red), the VG-20 parent (green), the FI (yellow), and the RILs (grey) at 24-25 days post-inoculation in the first (A) and second (B) screen. 


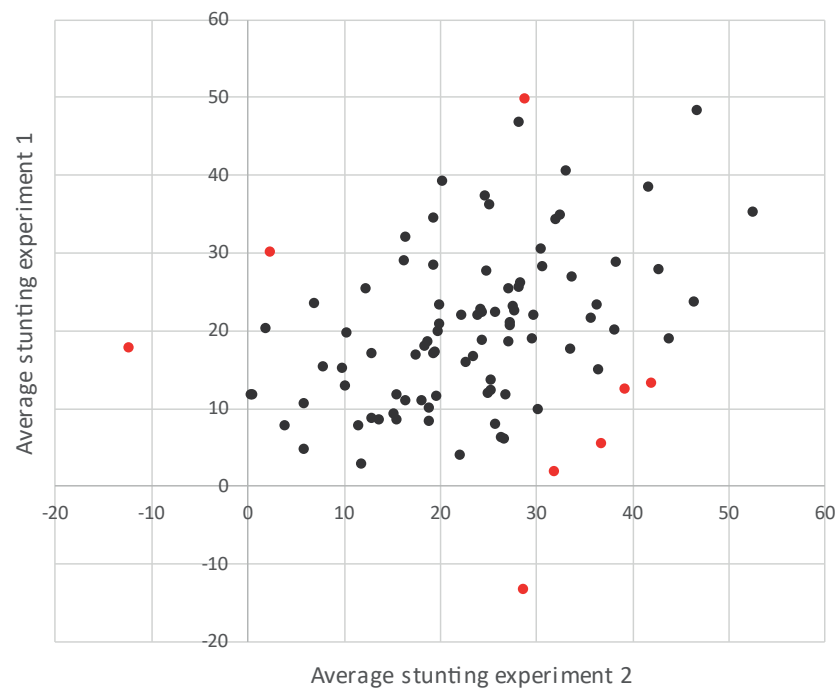

FIGURE 3 | Comparison of the average stunting of V. dahliae-inoculated RILs of the VG-20 x Moneymaker population (individual dots) in the first experiment (y-axis) and the second experiment ( $\mathrm{x}$-axis). Red dots represent the eight RILs with the largest variability in phenotype between the two experiments.

TABLE 1 | Estimates of the broad-sense heritability.

\begin{tabular}{cccc}
\hline Heritability $\mathbf{H}^{2}$ & Experiment 1 & Experiment 2 & Experiment 1+2 \\
\hline All 99 RILs & 0.21 & 0.16 & 0.15 \\
\hline $\begin{array}{c}\text { 91 RILs with most consistent } \\
\text { phenotype }\end{array}$ & 0.19 & 0.13 & 0.17 \\
\hline
\end{tabular}

$\mathrm{H}^{2}$ represents the broad-sense heritability of the stunting of $V$. dahliae-inoculated plants, with either all 99 RILs or only the 91 RILs after removal of the eight RILs with the largest variability in phenotype between the two screens. The broad-sense ANOVA was estimated using a one-way ANOVA.

\section{DNA marker analysis \& genetic map construction}

To construct a genetic map of the VG-20 population, all RILs were genotyped with a representative selection of 263 SNP-markers from the SolCap project (Sim et al. 2012). The selected markers were distributed across the genome with average intervals of $4 \mathrm{cM}$. To check the marker order on our map, we compared it with an extended version of the tomato EXPIMP 2009 map (Ashrafi et al. 2009). Although the genetic maps were comparable, some differences were observed. First of all, three wide gaps were observed on our genetic map on chromosome 5, 9 and 12 (Fig. 4, 6). These gaps were so wide that the markers flanking of these gaps could not be properly joined into the same linkage group (Fig. 4). Linkage groups are therefore referred to as chromosome 5.1, 5.2, 9.1, 9.2, 12.1 and 12.2. Although these gaps could also have been caused by a genotyping error, the gap on cho9 seems to be caused by a 
difference in crossover rates between the first markers on cho9 in the two populations. On the extended EXPIMP 2009 map, the distance between the two first markers on the distal portion of the short arm on cho9 was only $5 \mathrm{cM}$. In contrast, when the markers of cho9 were forced together in the same linkage group in the Moneymaker x VG-20 population, the distance between the first two markers was over 50 cM (Fig. 4, 6). The gaps on chromosome 5 and 12 are likely caused by a shortage of markers in those regions. For cho12, this was because of the high similarity of the genome regions between Moneymaker and VG-20, resulting in no available polymorphic SolCap markers. Besides the gaps on chromosome 5, 9 and 12 , we detected a strong segregation distortion on chromosome 2 (cho2; Fig. 5). For many of the markers on cho2, the allele of VG-20 was significantly overrepresented when compared with the Moneymaker allele. Furthermore, the physical order of three markers around the centromere on cho12 was different from the genetic order (Fig. 6). This was because of a mis-assembly in the cultivar 'Heinz' SL2.50 tomato genome, based on which the physical positions of the markers were determined, and which has been corrected in the recently updated SL4.0 tomato assembly (Table S3).

A

$\begin{array}{lllllllllllllll}1 & 2 & 3 & 4 & 5 & 6 & 7 & 8 & 9 & 10 & 11 & 12\end{array}$

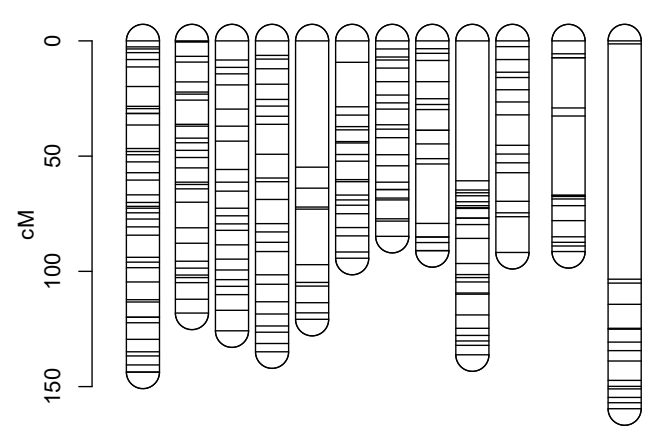

$\mathrm{B}$

$\begin{array}{lllllllllllllll}1 & 2 & 3 & 4 & 5.1 & 5.2 & 6 & 7 & 8 & 9.1 & 9.2 & 10 & 11 & 12.1 & 12.2\end{array}$
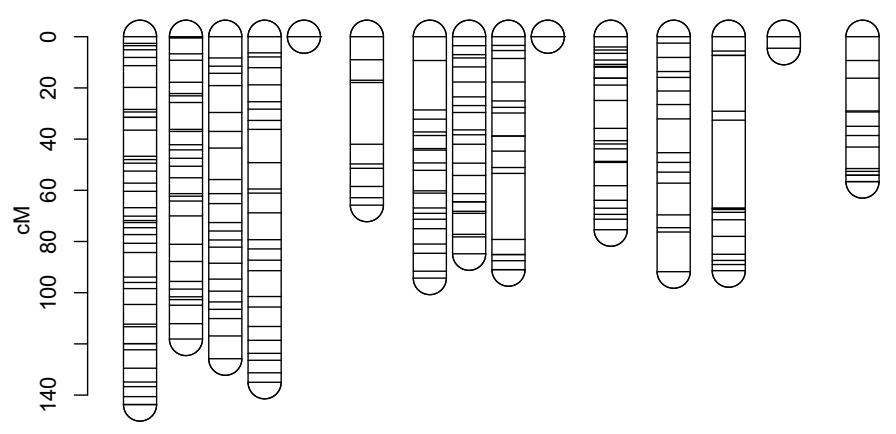

FIGURE 4 | Comparison of the genetic map of the Moneymaker x VG-20 RIL population, with the first marker on cho5 and the first two markers on chog forced in the same linkage group (A) or in separate linkage groups (B). Genetic maps were constructed using the R packages ASMap (Taylor and Butler 2017) and R/qtl (Broman 2010). 
Finally, the genetic distances on our map, regardless of the gaps on chromosome 5, 9 and 12, were generally larger than on the extended EXPIMP 2009 map. This could point towards higher crossover rates in our population compared with the population on which the EXPIMP 2009 population was based.

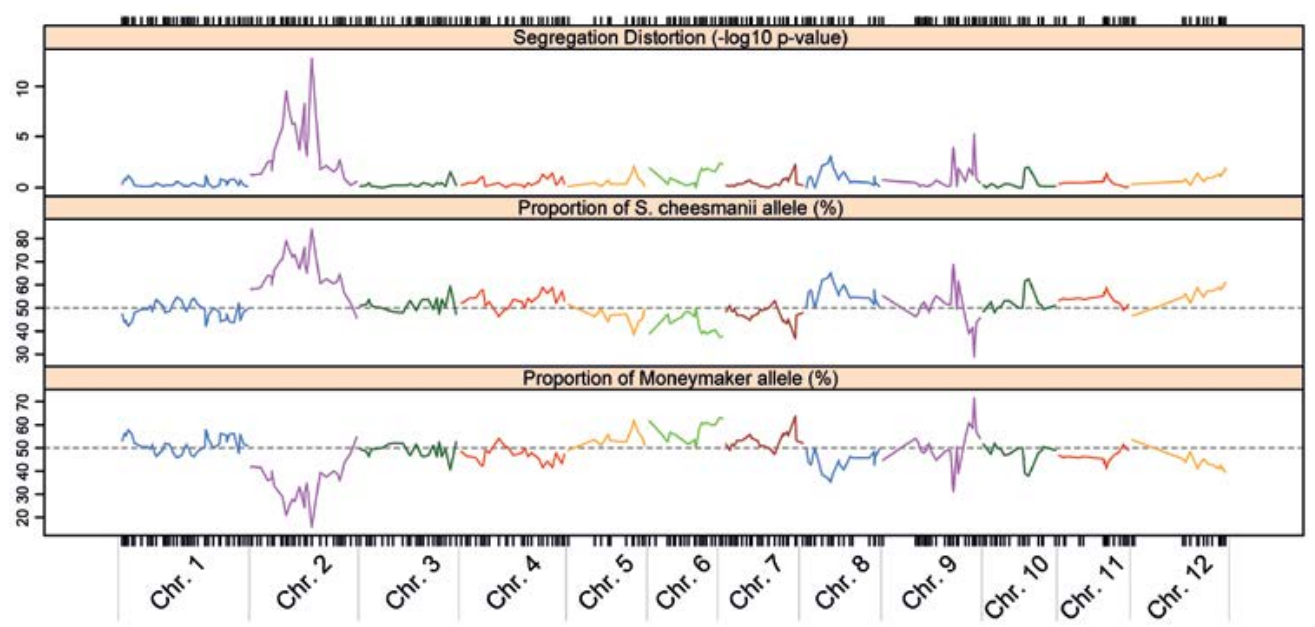

FICURE 5 | Overview of the coverage of the genomes of $S$. lycopersicum cultivar Moneymaker and VG-20 in the RIL population. The "Segregation distortion" track represents the -log10 p-values of a test of segregation distortion. The "Proportion" tracks represent the proportion of RILs with either the Moneymaker or the VG-20 allele for each marker. Black stripes on the $\mathrm{x}$-axis represent the genetic marker positions. The calculations and plotting were performed using the R package ASMap (Taylor and Butler 2017). 
Chromosome 1

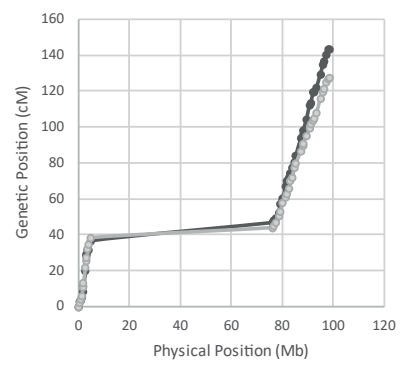

Chromosome 4

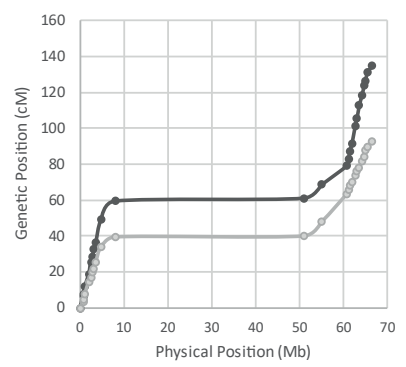

Chromosome 7

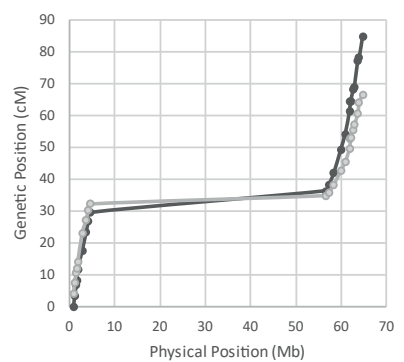

Chromosome 10

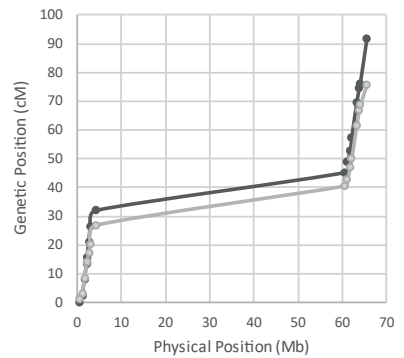

Chromosome 2

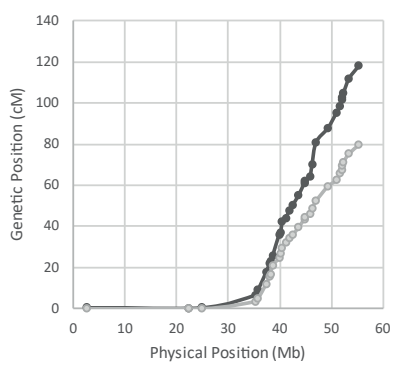

Chromosome 5

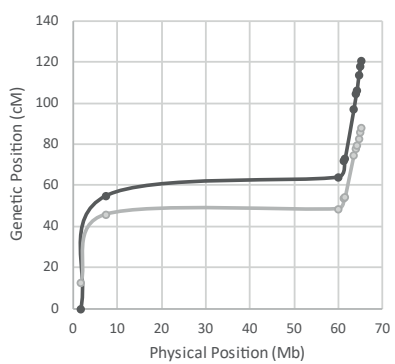

Chromosome 8

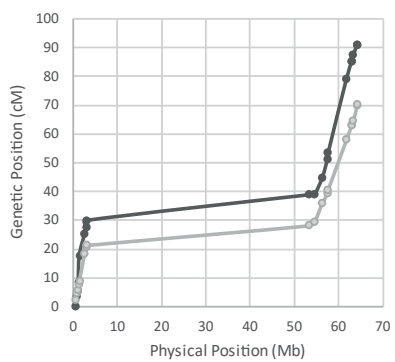

Chromosome 11

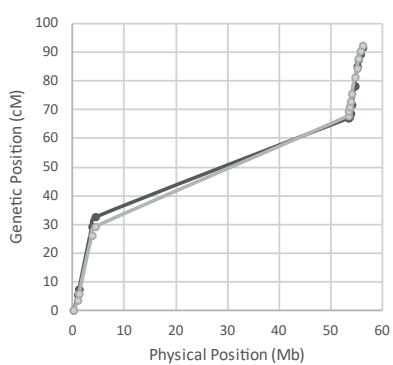

Chromosome 3

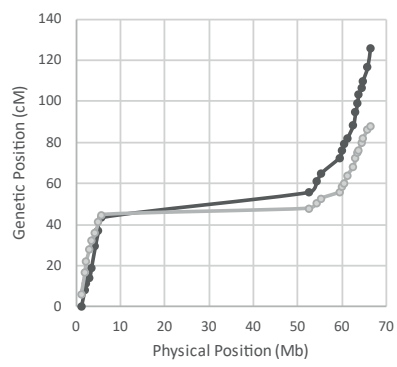

Chromosome 6

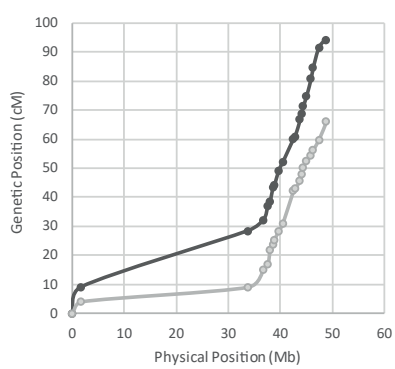

Chromosome 9

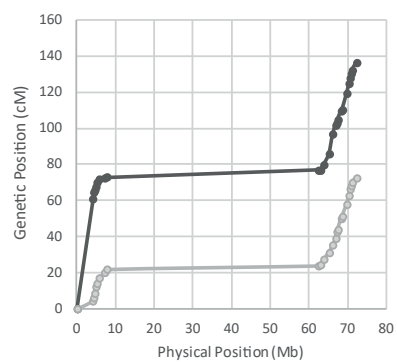

Chromosome 12

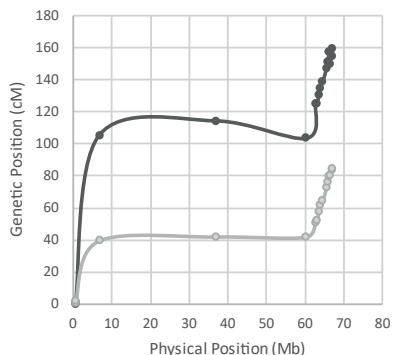

FIGURE 6 | Comparison of physical $(\mathrm{Mb})$ and genetic (cM) positions of the markers used to genotype the Moneymaker x VG-20 RIL population. Physical positions are based on version SL2.5 of the S. lycopersicum 'Heinz' reference genome. Grey dots and lines represent the positions of the EXPIMP 2009 map, black lines and dots represent the genetic positions of the markers in the Moneymaker x VG-20 RIL population. 


\section{Variation in canopy area affected the stunting of $V$. dahliae-inoculated plants}

Besides the stunting of $V$. dahliae-inoculated plants, the Moneymaker $x$ VG-20 RIL population also segregated for the canopy area of mock-inoculated plants. An interval mapping analysis for the canopy area of the mock-inoculated plants revealed a QTL at $46 \mathrm{cM}$ on chor (Fig. S3). Plants with the Moneymaker allele of this QTL displayed a larger canopy area than plants with the VG-20 allele (Fig S5). A comparison of the canopy area of the mock-inoculated plants with the stunting of $V$. dahliae-inoculated plants revealed a minor correlation (Fig. 7), suggesting that larger plants that are inoculated with $V$. dahliae are slightly more stunted than smaller plants that are inoculated with $V$. dahliae. To correct for this phenomenon, we included the canopy area of mock-inoculated plants as a covariate in the QTL analysis.

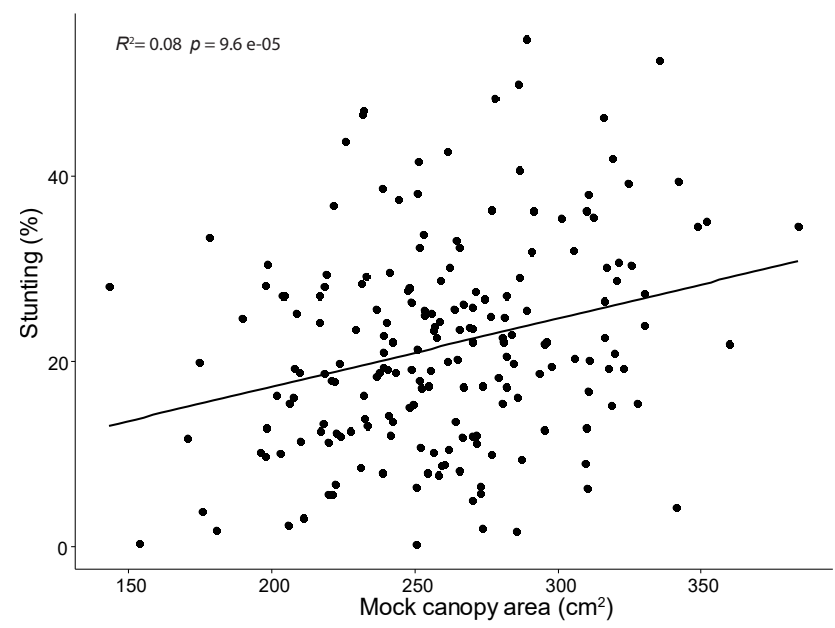

FICURE 7 | (A) Pearson Correlation analysis between the average canopy area of mock-inoculated plants and the average degree of stunting of the V. dahliae-inoculated RILs in the Moneymaker x VG-20 RIL population.

To identify QTLs for resistance against V. dahliae DVDS29 we used both interval mapping and a two-dimensional two-QTL scan from the R package R/QTL. However, based on the phenotypes of the two individual screens in the greenhouse, we could not identify significant or reproducible QTLs for stunting of $V$. dahliae-inoculated plants. Therefore, we combined the phenotypes from both experiments in one overall QTL analysis, separating the two experiments with a covariate. As a second covariate, we included the canopy area of mock-inoculated plants. Based on these analyses, we found evidence for QTLs on cho1, cho3, cho7 and cho9 (Figure 8, Table 2). Additionally, with these analyses we found indications for several other, less significant QTLs (Table S2). To further determine which combination of all these QTLs is best supported by our data, we performed several rounds of a stepwise selection method called "stepwiseqtl" from the package R/QTL. Based on this analysis, we confirmed that a model consisting of the QTLs on cho1, cho3, cho7 and 
cho9 are best supported by our data. The QTLs on cho1 and cho7 are contributed by VG-20, explaining $24.8 \%$ of the phenotypic variance (Table 3). Interestingly, the other two QTLS are contributed by Moneymaker, collectively explaining $16.52 \%$ of the phenotypic variance. The included covariates explained more of the phenotypic variance than the QTLs from Moneymaker, namely $17.4 \%$, with $11.4 \%$ for mock canopy area and $6 \%$ for the experiment number. Furthermore, the inclusion of these covariates resulted in higher LOD scores and a higher explained variance of the QTLs from VG-20 on cho1 and cho7 (Figure 8 A-B, Table 2). Finally, the QTL at $129.5 \mathrm{cM}$ on chor was only significant when the covariates were included.
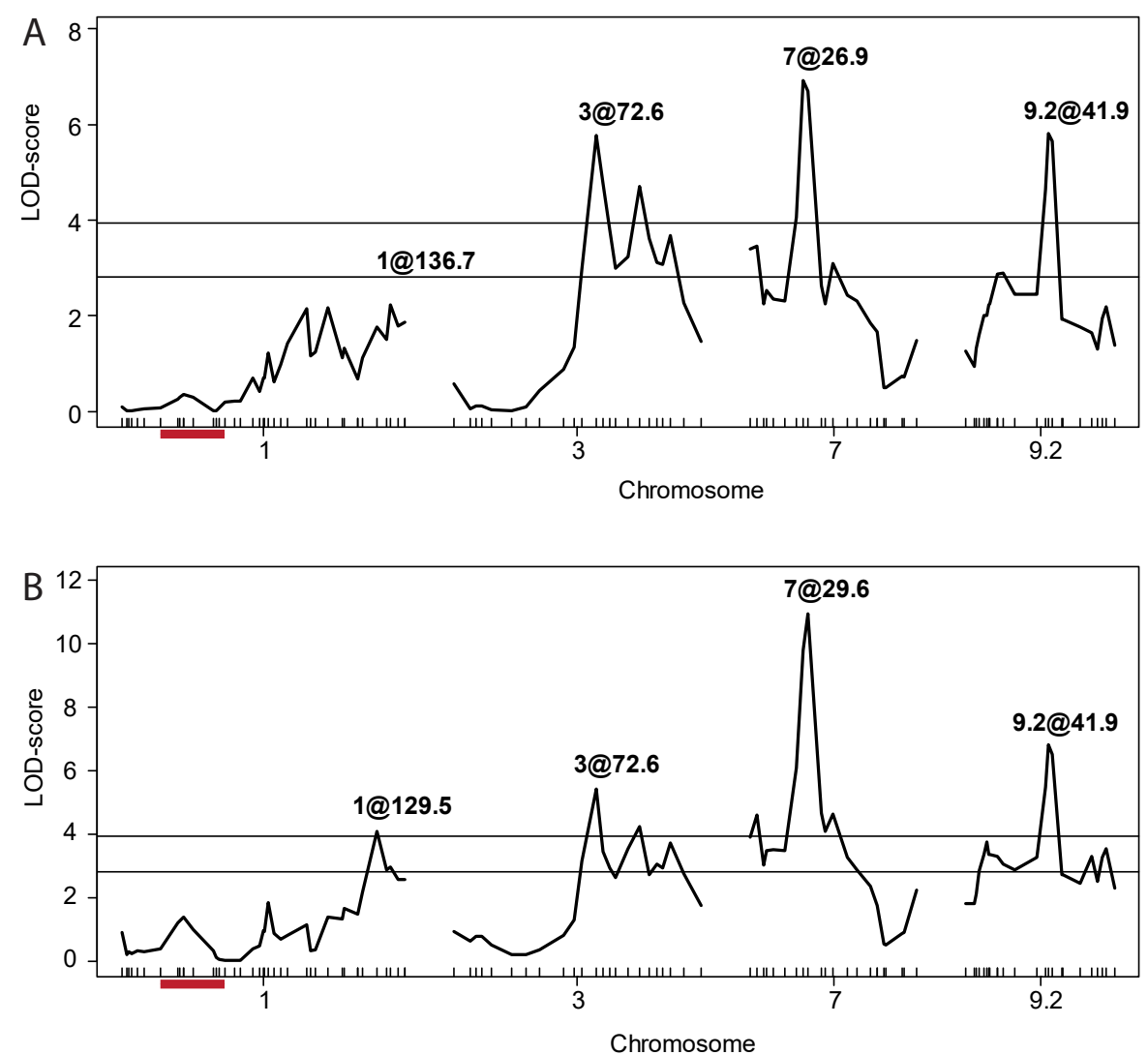

FIGURE 8 | The four most likely QTLs for V. dahliae resistance in the Moneymaker x VG-20 RIL population, estimated using "fitqtl" without (A) and with (B) the inclusion of the canopy area of mock-inoculated plants as a covariate. The Y-axis shows the LOD-scores, the $\mathrm{X}$-axis the position of the LOD-profiles. The lower horizontal black line represents the main-effect penalized LOD-threshold, the upper horizontal black line represents the strict interaction penalized LOD-threshold, determined with a two-dimensional permutation test (1000 permutations). The red horizontal line at cho1 indicates the position of the QTL for mock canopy area (Fig. S3). 
TABLE 2 | The most likely QTLs for resistance against V. dahliae DVDS29 with the inclusion of covariates.

\begin{tabular}{cccccccc}
\hline Chromosome & $\begin{array}{c}\text { Position } \\
\text { (cM) }\end{array}$ & $\begin{array}{c}\text { LOD } \\
\text { score }\end{array}$ & $\begin{array}{c}\text { Explained } \\
\text { variance }\end{array}$ & $\begin{array}{c}\text { Donor of } \\
\text { resistance } \\
\text { /tolerance }\end{array}$ & $\begin{array}{c}\text { LOD 1.5 } \\
\text { interval (cM) }\end{array}$ & $\begin{array}{c}\text { LOD 1.5 } \\
\text { interval (Mb) }\end{array}$ & $\begin{array}{c}\text { Number of } \\
\text { annotated genes } \\
\text { in interval }\end{array}$ \\
\hline 1 & 129.5 & $4.1(2.1)$ & $6.3(4.15)$ & VG-20 & $7.2(122.3-129.5)$ & $1.5(93.5-95)$ & $\sim 220$ \\
\hline 3 & 72.6 & $5.42(5.8)$ & $8.5(11.2)$ & Moneymaker & $51.7(65.2-116.9)$ & $10.4(55.5-65.9)$ & $\sim 870$ \\
\hline 7 & 29.6 & $10.9(6.9)$ & $18.5(13.7)$ & VG-20 & $12.9(23.5-36.4)$ & $52.8(3.8-56.6)$ & $\sim 870$ \\
\hline 9.2 & 41.9 & $6.8(5.8)$ & $10.9(11.3)$ & Moneymaker & $8.1(40.6-48.7)$ & $1.67(66.7-68.37)$ & $\sim 220$ \\
\hline
\end{tabular}

The four most likely QTLs for resistance against $V$. dahliae in the Moneymaker x VG-20 RIL population., estimated using "stepwiseqtl" without and without (in brackets) and with the inclusion of the canopy area of mock-inoculated plants and the experiment as a cofactor. The number of genes was estimated based on the physical positions of the flanking markers of the QTLs (LOD-1.5 interval) on the reference genome of S. lycopersicum 'Heinz' SL2.50. Gene annotations were based on the ITAG annotations version 2.5. Flanking markers of the LOD 1.5 interval can be found in Table Si.

\section{Three QTLs from VG-20 and two QTLs from Moneymaker caused reduced stunting symptoms}

To test how well the QTLs on chor and cho7 explain the resistance of VG-20 to V. dahliae DVDS29, we evaluated their effects on the stunting of $V$. dahliae-inoculated plants. As expected, RILs with the VG-20 allele of either the QTL on chor or cho7 displayed a reduction in stunting compared to RILs with the Moneymaker allele for both QTLs (Fig. 9A). Surprisingly, RILs with the VG-20 allele of the QTL for mock canopy area were less stunted than RILs with the Moneymaker allele of this QTL (Fig. 9B). This suggests that this QTL at 49 CM on cho1 may also affect resistance against $V$. dahliae DVDS29. When mock canopy area was not included as a covariate in the QTL analysis, we also found indications for a QTL for stunting at $46 \mathrm{cM}$ (Table S2, Table S5). It could thus be that the same QTL affects both V. dahliae-induced stunting and the canopy area of mock-inoculated plants, or alternatively, the two corresponding QTLs may be closely linked. Interestingly, only in RILs with the MM allele of the canopy area QTL, a significant difference in stunting was observed between RILs with the VG-20 allele and the MM allele of the stunting QTLs on chor and cho7 (Fig. 9B, panel 1-4). In RILs with the VG-20 allele of the canopy area QTL (Fig. 9B, panel 5-8), there was no significant difference in stunting between RILs with the VG-20 allele and the MM allele of the stunting QTLs on chor and cho7. Finally, even though RILs with the VG-20 allele of the QTLs on chor and cho7 were less stunted than RILs with the MM alleles, they were still more stunted than the VG-20 parent itself, which was on average $12.8 \%$ stunted (Fig. 9A-B). This suggests that these QTLs may not fully account for the resistance of VG-20. Similarly, RILs which possessed of the QTLs on chor and cho7 the Moneymaker allele were less stunted than Moneymaker (27.3\% stunting versus 45\% stunting) (Fig. 9A B).

Of the QTLs from Moneymaker, the QTL on ch03 and the QTL on cho9 appear to act additively, as RILs which possess of both QTLs the MM allele displayed the lowest level of 
stunting, whereas RILs which possessed of both QTLs the VG-20 allele displayed the highest level of stunting (Fig. 9C). Overall, we thus conclude that 3 QTLs from VG-20 and 2 QTLs from Moneymaker reduce the stunting of RILs challenged with the $V$. dahliae race 2 strain DVDS29.
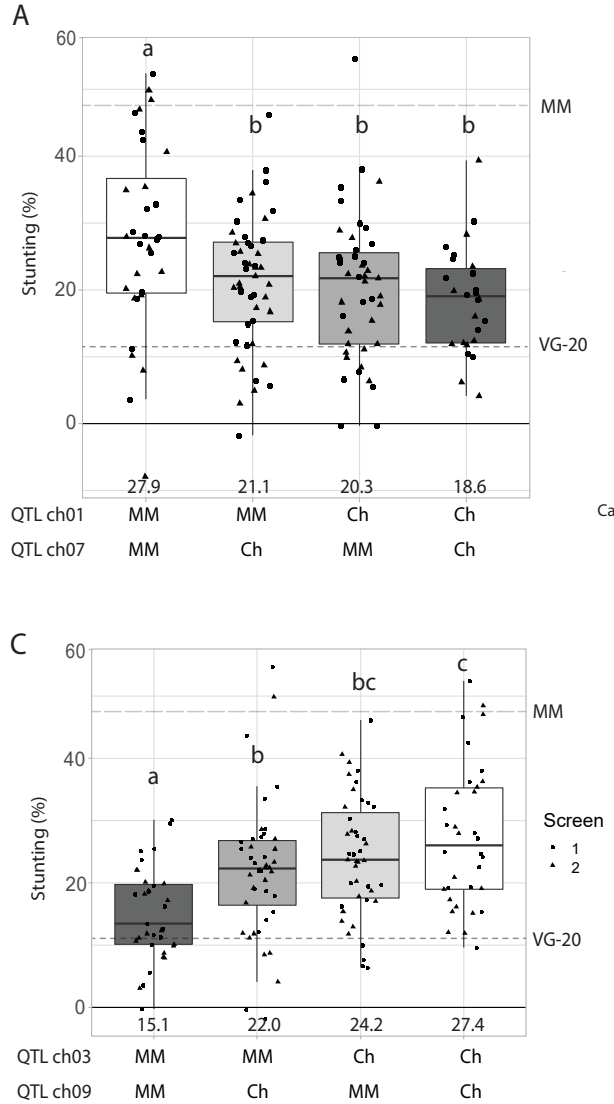

B

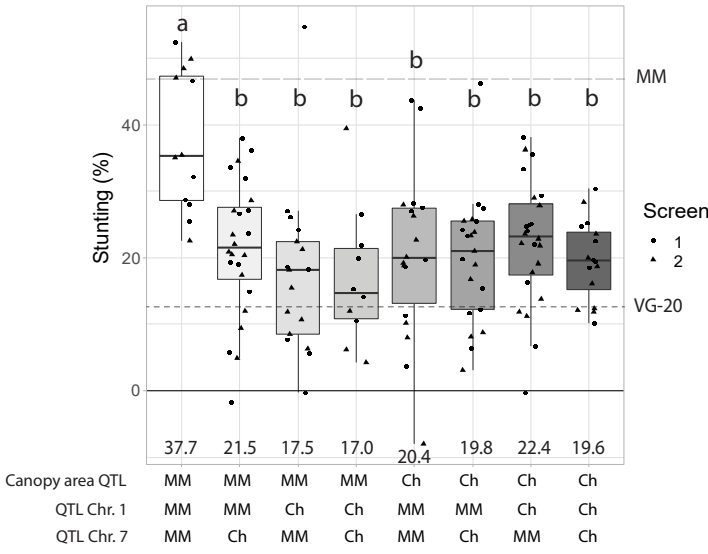

FIGURE 9 | Effects of the QTLs contributed by S. cheesmanii VG-2O (Ch) (A-B) and S. lycopersicum Moneymaker (MM) (C) on the average stunting of V. dahliae-inoculated plants (y-axis). (A) Effects of the QTLs on chor and cho7 from $S$. cheesmanii VG-20 on stunting. (B) Effects of the QTLs from S. cheesmanii VG-20 on chromosomes 1 and 7. "Canopy area QTL" represents the QTL on chor for mock canopy area. (C) Effects of the QTLs on cho3 and cho9 from S. cheesmanii VG-20 on stunting. The horizontal dashed line with large dashes represents the average stunting of Moneymaker (MM). Numbers under each boxplot indicate the average stunting of each group of RILs. Boxplots with different letter labels are significantly different according to a oneway ANOVA, followed by a Fisher's LSD test $(p<0.01)$.

\section{Discussion}

After the emergence of Verticillium wilt caused by $V$. dahliae race 2 strains on Ve-resistant tomato cultivars, breeders and researchers began a search for additional resistance sources in tomato. We previously identified three wild tomato accessions that displayed resistance against a collection of $V$. dahliae race 2 strains, of which especially VG-2O and $S$. pimpinellifolium VG-21 showed the strongest resistance. In this chapter, we demonstrated that the resistance of VG-20 to $\mathrm{V}$. dahliae segregates quantitatively and is dominantly inherited. We identified two QTLs of which the allele from VG-20 reduced the stunting of 
plants inoculated with V. dahliae DVDS29. Furthermore, we identified 2 QTLs of which the allele from Moneymaker reduced the stunting of plants inoculated with $V$. dahliae DVDS29.

When segregation for other traits affects the variance of the trait of interest, this may affect the success of QTL analysis. Therefore, when mapping QTLs, it can be helpful to include these traits as covariates in the analysis. If a covariate affects the phenotype, including it will reduce the residual variation, and thereby improve the QTL analysis (Broman and Sen 2009). In our analysis, we included the size of the mock-inoculated plants as one covariate, as genotypes with larger mock-inoculated plants were somewhat more stunted when inoculated with $V$. dahliae DVDS29 (Fig. 7). Because we combined the phenotypes of two experiments in one analysis, we included the experiment number as another covariate. Including these covariates visibly affected the QTL analysis, as the LOD-scores and the explained variance of the QTLs from VG-20 increased, and it led to the identification of an additional QTL on chor.

Inclusion of the covariates also caused some QTLs to disappear, including a QTL on chor that overlapped with a QTL associated with the canopy area of mock-inoculated plants (Fig. S3). Given that we demonstrated that the canopy area of mock-inoculated plants affects the degree of stunting of $V$. dahliae inoculated plants (Fig. 7), these QTLs may thereby be an indirect effect of the variation in plant size, and therefore not QTLs for resistance against V. dahliae DVDS29. This would then explain why these QTLs disappeared upon inclusion of the covariates in the QTL analysis. However, our data demonstrated that plant size and resistance against $V$. dahliae DVDS29 are not necessarily unlinked traits in this RIL population. On cho1, a QTL for plant size was found at a similar position at as a QTL for resistance against $V$. dahliae DVDS29 (Table S2, Fig. S3), at 49 and $46 \mathrm{cM}$, respectively. Inclusion of the covariate for plant size resulted in the disappearance of this QTL (Table S5 vs Table S6). Still, V. dahliae-inoculated genotypes with the VG-20 allele of this QTL were less stunted than $V$. dahliae-inoculated genotypes with Moneymaker allele. It seems unlikely that this reduction in stunting is caused by the slight difference in size between plants with the different alleles of the QTL for plant size (Fig. S5). This thus suggests that this QTL is involved in resistance against $V$. dahliae DVDS29. This would also explain why the QTL on cho1 at $139 \mathrm{cM}$ and cho7 only affect the degree of stunting in the Moneymaker background of the other QTL (Fig 9B). After all, if this QTL on cho1 at $46 \mathrm{cM}$ would sufficiently reduce the degree of stunting, the effect of the other resistance QTLs on chol and cho7 may no longer be visible. The fact that the region at $46-49 \mathrm{cM}$ on chor is associated with both $V$. dahliae symptoms and plants size could then be because the resistance QTL from VG-20 could be genetically linked to a QTL for plant size. Alternatively, the resistance of this QTL may come with a cost to the growth of the plants. This is not is uncommon, as more resistances were found to come with a cost on plant growth or yield (Brown 2003, 2002). 
Both when a resistance QTL is linked to a QTL for plant size or whether the resistance comes with a cost to plant growth, the inclusion of the covariate will hamper the analysis. After all, any QTLs for resistance that also affect plant size - or are linked to such QTL - will not be detected when the covariate is included. Still, the inclusion of the covariate did result in the identification of an additional QTL and increase the LOD score and the explained variance of the QTLs. Therefore, it seems that it depends on the QTL whether inclusion of a covariate improves the QTL analysis. A QTL analysis for resistance against V. dahliae should therefore be performed both with and without the covariate so the results can be compared.

Although based on this analysis, we provided evidence for three QTLs for the resistance of VG-20, this QTL analysis is only the first step. As the QTLs we identified could only be found when the phenotypes from both RIL screens were combined into one analysis, additional research must be performed to confirm the results. First of all, increasing the number of genetic markers may help get more precise estimates of the QTL regions, and further narrow down these QTLs. Although more markers will not improve the reliability of the phenotypes, is may help identify additional QTLs we missed in our analysis. As there are no markers covering the big gaps on the genetic map on chromosome 5, 9 and 12, QTLs in these chromosomal regions may have gone undetected. Therefore, additional markers for these regions would be beneficial. Also, the power of our QTL analysis was limited since only 91 RILs were used. To reliably map quantitative traits, many studies showed that a population of multiple hundreds of genotypes is desirable (Li et al. 2010; Vales et al. 2005). Thus, both increasing the number of markers and the number of RILs may help identify additional, undetected QTLs, and help to further confirm the QTLs that were identified in this chapter. Finally, we detected a severe segregation distortion on chromosome 2, as for most markers on this chromosome, the allele of VG-20 was overrepresented. Any QTLs on cho2 may therefore not have been detected. Therefore, enriching the RIL population with additional RILs which possess the Moneymaker allele of these markers on cho2 may help detect any QTLs on cho2 which have gone unnoticed.

However, to fully confirm the QTLs from VG-20 on chor and cho7, more is needed than testing additional RILs and markers. The best way to confirm the QTLs would be to develop near-isogenic lines (NILs) that are isogenic for most of the genome but differ for the QTL of interest. In this way, the effect of the QTLs can be confirmed in a fixed genetic background. Two approaches can be used to develop these NILs. Firstly, as proposed by Tuinstra et al. (1997), NILs can be developed directly from the RIL population itself, using RILs which are still heterozygous for the QTL of interest, but homozygous for the rest of the genome. Selfing these RILs would result in a Heterogeneous Inbred Family (HIF), which segregates for the QTL while the rest of the genome is fixed. However, one would need to find a RIL which lacks the other resistance QTLs from VG-20, to optimally see an effect of the QTL of interest. In our current population, such RILs do not exist for all QTLs of interest. Another popular 
strategy to develop NILs is by backcrossing. This can be done by crossing RILs harbouring the QTL from VG-20 back to Moneymaker. After each round of backcrossing, the offspring should then be genotyped with several markers covering the QTL, to select the genotypes with the VG-20 allele of the QTL to further cross back to Moneymaker. After several rounds of backcrossing, NILs will be formed with most of their genome from Moneymaker, except for the QTL regions. If these NILs, when infected with V. dahliae DVDS29, are indeed less stunted than Moneymaker, this would confirm the QTLs. Furthermore, comparing NILs which have of all the QTLs on chor and cho7 the VG-20 allele with NILs which have of only one or two of the QTLs the VG-20 allele should reveal whether or not there is an additive effect between these two QTLs. Similarly, comparing the symptom expression of these NILs with the VG-20 parents would validate whether these QTLs fully account for the resistance of VG-20, or whether additional QTLs are required to reach the resistance level of VG-20. Finally, although we found a reduction in $V$. dahliae DVDS29 colonization in inoculated VG-20 plants (chapter 3), it is unclear whether the QTLs from VG-20 also reduce the colonization of $V$. dahliae DVD29. In other words, we cannot yet distinguish whether these QTLs are for resistance or for tolerance to V. dahliae DVDS29. Therefore, the colonization of $V$. dahliae DVDS29 in NILs should be compared with the colonization in Moneymaker plants to confirm whether the QTLs on cho1 and cho7 are true resistance QTLs.

Besides the QTLs from VG-20, two QTLs associated with stunting symptoms were found to come from Moneymaker. Although Moneymaker is universally used as a susceptible control in $V$. dahliae experiments, the identification of QTLs from a susceptible parent is not uncommon, as several other mapping studies for $V$. dahliae resistance also report this (Fang et al. 2014; Rygulla et al. 2008; Wang et al. 2008; Zhang et al. 2014b). Interestingly, in the S. pimpinellifolium VG-3 RIL population, we also found a QTL from Moneymaker associated with reduced Verticillium wilt symptoms on cho3. However, it is rather striking that in the VG-3 RIL population, this QTL could only be identified based on the number of yellowing leaves of $V$. dahliae-inoculated plants and not based on stunting. Furthermore, in the VG20 RIL population, three peaks were observed on cho3 in contrast to one large peak in the VG-3 RIL population. Whether these are therefore the same QTLs cannot be conclusively determined based on our current data. Still, these results demonstrate that Moneymaker possesses some level of defence against $V$. dahliae, with a different genetic basis than the resistance of VG-3 and VG-20.

Many of the mapping studies that attempted to identify the Ver gene are based on both the stunting and foliar symptoms such as chlorosis, yellowing, and necrosis (Kawchuk et al. 2001; Lynch et al. 1997; Use et al. 1953). Similar to the VG-21 RIL population, several RILs of the VG-20 population developed yellowing and necrosis on the lower leaves in the absence of $V$. dahliae inoculation (Fig. S1), making yellowing of leaves an unreliable Verticillium wilt symptom in these populations. We could therefore not confirm whether the QTLs from VG- 
20 also affect the number of yellowing leaves. Similar to the VG-3 and VG-21 RIL-populations, the estimated heritability of the stunting phenotype was low. Although these are only rough estimates of heritability, it suggests that only a small proportion of the phenotypic variance is due to genetic factors. Given the typically high degree of variance in $V$. dahliae symptoms, this is not unexpected. As we already assessed the optimal methods for inoculation and phenotyping (chapter 2 ) we do not see further approaches which will drastically reduce the variation in $V$. dahliae symptoms to improve the estimated heritability. However, further increasing the number of replicates per genotypes may result in more reliable estimates of the phenotypes, which may improve the QTL analysis. Still, this will also substantially increase the size of the experiments. Especially when both the number of RILs and the number of replicates per RIL is increased, this will result into an experiment consisting of up to many thousands of plants, making the practical feasibility of such an experiment challenging. Therefore, confirming the QTLs we present in this chapter, combined with increasing the number of RILs and markers to search for additional QTLs should be the first steps forward.

From a scientific point of view, further studying and confirming both the QTLs from VG20 and Moneymaker will have its relevance. After all, identifying the causal genes and mechanisms for the resistance or tolerance of Moneymaker could also result in novel insights in how plants cope with disease caused by $V$. dahliae. However, from a plant breeding point of view, the first and primary focus should be the confirmation of the QTLs from VG-20. After all, we show in chapter 3 that VG-20 is more resistant than Moneymaker. Especially the QTLs from VG-20 could thus help with the development of more resistant cultivars to control V. dahliae. Finally, it should be determined whether multiple QTLs are required to reach a satisfactory level of resistance, or whether introgression of only one of the QTLs on chor and cho7 is sufficient. 


\section{Supplemental material}

SUPPLEMENTAL TABLE 1 | Markers used for the QTL analysis and genetic map construction. Genetic positions represent the genetic map of the Moneymaker x VG-20 RIL population. Markers were used from Solanaceae Coordinated Agricultural Project (2019) and Sim et al. (2012).

\begin{tabular}{|c|c|c|c|c|c|c|}
\hline Marker name & Chromosome & $\begin{array}{c}\text { Linkage } \\
\text { group }\end{array}$ & $\begin{array}{c}\text { Genetic } \\
\text { Position } \\
\text { (cM) }\end{array}$ & $\begin{array}{c}\text { Physical } \\
\text { position } \\
(\mathrm{Mb})\end{array}$ & $\begin{array}{c}\text { Moneymaker } \\
\text { SNP variant }\end{array}$ & $\begin{array}{c}\text { VG-20 SNP } \\
\text { variant }\end{array}$ \\
\hline solcap_snp_sl_15058 & 1 & 1 & 0.00 & 45434 & A & G \\
\hline solcap_snp_sl_33745 & 1 & 1 & 2.63 & 534448 & G & $\mathrm{T}$ \\
\hline solcap_snp_sl_33737 & 1 & 1 & 3.46 & 853700 & G & A \\
\hline solcap_snp_sl_20499 & 1 & 1 & 5.13 & 1116348 & $\mathrm{~T}$ & A \\
\hline solcap_snp_sl_60303 & 1 & 1 & 8.15 & 1605688 & C & $\mathrm{T}$ \\
\hline solcap_snp_sl_60276 & 1 & 1 & 11.31 & 1809372 & A & G \\
\hline solcap_snp_sl_8640 & 1 & 1 & 19.79 & 2554370 & A & G \\
\hline solcap_snp_sl_60089 & 1 & 1 & 28.42 & 2879156 & A & G \\
\hline solcap_snp_sl_60043 & 1 & 1 & 29.41 & 3101943 & C & $\mathrm{T}$ \\
\hline solcap_snp_sl_59964 & 1 & 1 & 31.52 & 3591306 & C & $\mathrm{T}$ \\
\hline solcap_snp_sl_20438 & 1 & 1 & 31.52 & 3899743 & $\mathrm{~T}$ & C \\
\hline solcap_snp_sl_33677 & 1 & 1 & 36.52 & 4871337 & G & $\mathrm{T}$ \\
\hline solcap_snp_sl_17073 & 1 & 1 & 72.56 & 74481568 & G & A \\
\hline solcap_snp_sl_30135 & 1 & 1 & 46.72 & 76357382 & A & G \\
\hline solcap_snp_sl_50470 & 1 & 1 & 48.03 & 76778320 & A & C \\
\hline solcap_snp_sl_50427 & 1 & 1 & 49.35 & 77359528 & G & A \\
\hline solcap_snp_sl_38078 & 1 & 1 & 52.48 & 78576993 & C & $\mathrm{T}$ \\
\hline solcap_snp_sl_25931 & 1 & 1 & 57.16 & 79245417 & C & $\mathrm{T}$ \\
\hline solcap_snp_sl_12352 & 1 & 1 & 60.35 & 80147347 & $\mathrm{~T}$ & C \\
\hline solcap_snp_sl_36963 & 1 & 1 & 66.84 & 81331189 & $\mathrm{~T}$ & G \\
\hline solcap_snp_sl_27345 & 1 & 1 & 70.06 & 81888731 & G & A \\
\hline solcap_snp_sl_1813 & 1 & 1 & 71.82 & 82521565 & A & $\mathrm{T}$ \\
\hline solcap_snp_sl_27600 & 1 & 1 & 74.64 & 83237569 & $\mathrm{~T}$ & C \\
\hline SL10943_357 & 1 & 1 & 77.34 & 83829584 & G & $\mathrm{T}$ \\
\hline solcap_snp_sl_34513 & 1 & 1 & 80.74 & 84767919 & A & G \\
\hline solcap_snp_sl_15339 & 1 & 1 & 84.26 & 85351601 & $\mathrm{~T}$ & C \\
\hline solcap_snp_sl_28173 & 1 & 1 & 93.88 & 87334152 & C & $\mathrm{T}$ \\
\hline solcap_snp_sl_43786 & 1 & 1 & 95.99 & 88005386 & G & $\mathrm{T}$ \\
\hline solcap_snp_sl_43843 & 1 & 1 & 98.39 & 88239598 & C & $\mathrm{T}$ \\
\hline solcap_snp_sl_28287 & 1 & 1 & 104.61 & 89572247 & C & $\mathrm{T}$ \\
\hline solcap_snp_sl_2504 & 1 & 1 & 112.27 & 90662089 & G & A \\
\hline solcap_snp_sl_40011 & 1 & 1 & 113.30 & 91224236 & C & A \\
\hline solcap_snp_sl_40219 & 1 & 1 & 119.89 & 92153144 & A & G \\
\hline solcap_snp_sl_40309 & 1 & 1 & 119.89 & 92492885 & A & C \\
\hline
\end{tabular}




\begin{tabular}{|c|c|c|c|c|c|c|}
\hline Marker name & Chromosome & $\begin{array}{c}\text { Linkage } \\
\text { group }\end{array}$ & $\begin{array}{c}\text { Genetic } \\
\text { Position } \\
(\mathrm{cM})\end{array}$ & $\begin{array}{c}\text { Physical } \\
\text { position } \\
(\mathrm{Mb})\end{array}$ & $\begin{array}{l}\text { Moneymaker } \\
\text { SNP variant }\end{array}$ & $\begin{array}{l}\text { VG-20 SNP } \\
\text { variant }\end{array}$ \\
\hline solcap_snp_sl_26879 & 1 & 1 & 122.30 & 93475357 & A & $\mathrm{T}$ \\
\hline solcap_snp_sl_19481 & 1 & 1 & 129.51 & 94950172 & A & G \\
\hline C2_At2g15890_430_b & 1 & 1 & 134.86 & 96037443 & $\mathrm{~T}$ & A \\
\hline solcap_snp_sl_48209 & 1 & 1 & 136.74 & 96508829 & G & A \\
\hline solcap_snp_sl_48077 & 1 & 1 & 140.63 & 97173206 & $\mathrm{~T}$ & C \\
\hline $\begin{array}{l}\text { SGN-U581138_snp1171_solcap_snp_ } \\
\text { sl_53721 }\end{array}$ & 1 & 1 & 143.71 & 97994925 & G & $\mathrm{C}$ \\
\hline solcap_snp_sl_53612 & 1 & 1 & 143.71 & 98411966 & A & G \\
\hline solcap_snp_sl_31191 & 2 & 2 & 0.00 & 22214295 & $\mathrm{~T}$ & C \\
\hline solcap_snp_sl_5881 & 2 & 2 & 0.40 & 24789250 & G & $\mathrm{T}$ \\
\hline solcap_snp_sl_11804 & 2 & 2 & 0.40 & 2557746 & G & $\mathrm{T}$ \\
\hline solcap_snp_sl_26136 & 2 & 2 & 6.68 & 35274016 & $\mathrm{~T}$ & C \\
\hline solcap_snp_sl_36484 & 2 & 2 & 9.19 & 35735971 & A & G \\
\hline solcap_snp_sl_8402 & 2 & 2 & 17.81 & 37304557 & G & A \\
\hline Leoo5019_205 & 2 & 2 & 22.19 & 37951739 & $\mathrm{~T}$ & C \\
\hline solcap_snp_sl_14951 & 2 & 2 & 23.07 & 38129529 & C & $\mathrm{T}$ \\
\hline solcap_snp_sl_8464 & 2 & 2 & 25.73 & 38544729 & C & A \\
\hline solcap_snp_sl_33636 & 2 & 2 & 36.18 & 39810804 & G & A \\
\hline solcap_snp_sl_10557 & 2 & 2 & 37.00 & 40105644 & A & C \\
\hline CLoo9186-0301_solcap_snp_sl_35943 & 2 & 2 & 42.20 & 40408829 & $\mathrm{C}$ & $\mathrm{T}$ \\
\hline solcap_snp_sl_25477 & 2 & 2 & 44.24 & 41109178 & A & $\mathrm{T}$ \\
\hline solcap_snp_sl_25398 & 2 & 2 & 47.55 & 41731120 & $\mathrm{~T}$ & $\mathrm{C}$ \\
\hline solcap_snp_sl_13612 & 2 & 2 & 50.62 & 42465443 & $\mathrm{~T}$ & C \\
\hline CLo16725-0417 & 2 & 2 & 55.15 & 43559167 & $\mathrm{~T}$ & G \\
\hline solcap_snp_sl_49480 & 2 & 2 & 61.33 & 44700158 & G & A \\
\hline solcap_snp_sl_49505 & 2 & 2 & 62.25 & 44772753 & $\mathrm{~T}$ & A \\
\hline solcap_snp_sl_23909 & 2 & 2 & 64.23 & 45761358 & $\mathrm{~T}$ & C \\
\hline CLo15119-0265_solcap_snp_sl_49752 & 2 & 2 & 70.04 & 46177219 & A & $\mathrm{T}$ \\
\hline solcap_snp_sl_49928 & 2 & 2 & 81.08 & 46970648 & $\mathrm{~T}$ & C \\
\hline solcap_snp_sl_12841 & 2 & 2 & 87.80 & 49223988 & $\mathrm{C}$ & $\mathrm{T}$ \\
\hline solcap_snp_sl_36287 & 2 & 2 & 95.55 & 50965815 & C & $\mathrm{T}$ \\
\hline solcap_snp_sl_67184 & 2 & 2 & 98.61 & 51626874 & $\mathrm{~T}$ & C \\
\hline solcap_snp_sl_67119 & 2 & 2 & 101.62 & 51919149 & G & C \\
\hline solcap_snp_sl_67090 & 2 & 2 & 102.72 & 52064928 & $\mathrm{C}$ & A \\
\hline CL017715-0287_solcap_snp_sl_67052 & 2 & 2 & 104.94 & 52277651 & G & A \\
\hline solcap_snp_sl_21867 & 2 & 2 & 112.10 & 53371077 & G & A \\
\hline solcap_snp_sl_47880 & 2 & 2 & 118.12 & 55238304 & $\mathrm{~T}$ & G \\
\hline solcap_snp_sl_9681 & 3 & 3 & 0.00 & 1257561 & $\mathrm{~T}$ & C \\
\hline solcap_snp_sl_9689 & 3 & 3 & 8.27 & 2053015 & C & $\mathrm{T}$ \\
\hline solcap_snp_sl_9703 & 3 & 3 & 11.50 & 2372139 & $\mathrm{~T}$ & A \\
\hline
\end{tabular}




\begin{tabular}{|c|c|c|c|c|c|c|}
\hline Marker name & Chromosome & $\begin{array}{l}\text { Linkage } \\
\text { group }\end{array}$ & $\begin{array}{c}\text { Genetic } \\
\text { Position } \\
(\mathrm{cM})\end{array}$ & $\begin{array}{c}\text { Physical } \\
\text { position } \\
(\mathrm{Mb})\end{array}$ & $\begin{array}{l}\text { Moneymaker } \\
\text { SNP variant }\end{array}$ & $\begin{array}{l}\text { VG-20 SNP } \\
\text { variant }\end{array}$ \\
\hline $\begin{array}{l}\text { SGN-U564013_snp1820_solcap_snp_ } \\
\text { sl_55020 }\end{array}$ & 3 & 3 & 14.29 & 3138305 & G & A \\
\hline solcap_snp_sl_19509 & 3 & 3 & 19.08 & 3548679 & $\mathrm{C}$ & G \\
\hline solcap_snp_sl_23193 & 3 & 3 & 29.61 & 4427906 & A & $\mathrm{T}$ \\
\hline Leooo209_237_solcap_snp_sl_26315 & 3 & 3 & 37.01 & 5136629 & A & G \\
\hline solcap_snp_sl_26366 & 3 & 3 & 43.54 & 5878593 & A & G \\
\hline solcap_snp_sl_30678 & 3 & 3 & 55.78 & 52628897 & G & A \\
\hline solcap_snp_sl_52395 & 3 & 3 & 61.30 & 54449864 & A & $\mathrm{C}$ \\
\hline solcap_snp_sl_18982 & 3 & 3 & 65.22 & 55485943 & G & A \\
\hline solcap_snp_sl_10377 & 3 & 3 & 72.63 & 59506958 & $\mathrm{~T}$ & C \\
\hline solcap_snp_sl_21694 & 3 & 3 & 75.92 & 60059066 & A & G \\
\hline solcap_snp_sl_35697 & 3 & 3 & 79.41 & 60662256 & A & G \\
\hline solcap_snp_sl_19654 & 3 & 3 & 82.21 & 61330355 & $\mathrm{~T}$ & $\mathrm{C}$ \\
\hline solcap_snp_sl_11602 & 3 & 3 & 88.48 & 62646622 & $\mathrm{~T}$ & C \\
\hline CLo15346-0143_solcap_snp_sl_62495 & 3 & 3 & 94.65 & 63163824 & G & C \\
\hline CLO17416-0406 & 3 & 3 & 99.44 & 63618158 & $\mathrm{~T}$ & G \\
\hline solcap_snp_sl_62348 & 3 & 3 & 103.60 & 63841826 & G & $\mathrm{T}$ \\
\hline solcap_snp_sl_62228 & 3 & 3 & 106.47 & 64485318 & A & G \\
\hline CLo15369-0414 & 3 & 3 & 110.06 & 64823443 & C & $\mathrm{T}$ \\
\hline CLo17760-0371_solcap_snp_sl_61928 & 3 & 3 & 116.90 & 65888518 & $\mathrm{~T}$ & G \\
\hline solcap_snp_sl_61847 & 3 & 3 & 125.78 & 66463126 & C & $\mathrm{T}$ \\
\hline solcap_snp_sl_45224 & 4 & 4 & 0.00 & 35288 & $\mathrm{~T}$ & C \\
\hline solcap_snp_sl_9832 & 4 & 4 & 6.30 & 741558 & A & $\mathrm{T}$ \\
\hline solcap_snp_sl_9856 & 4 & 4 & 7.86 & 845964 & A & G \\
\hline solcap_snp_sl_63869 & 4 & 4 & 12.10 & 1163761 & $\mathrm{~T}$ & G \\
\hline solcap_snp_sl_21335 & 4 & 4 & 18.78 & 2050817 & C & $\mathrm{T}$ \\
\hline solcap_snp_sl_34675 & 4 & 4 & 25.41 & 2470709 & A & G \\
\hline solcap_snp_sl_21372 & 4 & 4 & 28.32 & 2915842 & C & $\mathrm{T}$ \\
\hline solcap_snp_sl_34684 & 4 & 4 & 32.71 & 3174985 & A & G \\
\hline solcap_snp_sl_21390 & 4 & 4 & 36.21 & 3636527 & $\mathrm{~T}$ & C \\
\hline solcap_snp_sl_27167 & 4 & 4 & 49.16 & 4832005 & G & $\mathrm{C}$ \\
\hline solcap_snp_sl_45951 & 4 & 4 & 59.46 & 7963140 & C & $\mathrm{T}$ \\
\hline solcap_snp_sl_45378 & 4 & 4 & 61.06 & 50846174 & G & $\mathrm{T}$ \\
\hline solcap_snp_sl_24606 & 4 & 4 & 68.81 & 55046199 & G & $\mathrm{T}$ \\
\hline solcap_snp_sl_36796 & 4 & 4 & 79.33 & 60700555 & G & A \\
\hline solcap_snp_sl_17922 & 4 & 4 & 82.88 & 61100688 & G & $\mathrm{C}$ \\
\hline CLo17841-0157 & 4 & 4 & 87.35 & 61295959 & $\mathrm{~T}$ & G \\
\hline solcap_snp_sl_17956 & 4 & 4 & 91.43 & 61799612 & $\mathrm{~T}$ & $\mathrm{C}$ \\
\hline CL016270-0427_solcap_snp_sl_47056 & 4 & 4 & 101.48 & 62550134 & G & A \\
\hline SGN-U594049_snp94598 & 4 & 4 & 105.64 & 62958495 & G & A \\
\hline
\end{tabular}




\begin{tabular}{|c|c|c|c|c|c|c|}
\hline Marker name & Chromosome & $\begin{array}{l}\text { Linkage } \\
\text { group }\end{array}$ & $\begin{array}{c}\text { Genetic } \\
\text { Position } \\
\text { (cM) }\end{array}$ & $\begin{array}{c}\text { Physical } \\
\text { position } \\
(\mathrm{Mb})\end{array}$ & $\begin{array}{c}\text { Moneymaker } \\
\text { SNP variant }\end{array}$ & $\begin{array}{c}\text { VG-20 SNP } \\
\text { variant }\end{array}$ \\
\hline CLo17321-0298_solcap_snp_sl_47277 & 4 & 4 & 113.23 & 63497531 & A & G \\
\hline solcap_snp_sl_47439 & 4 & 4 & 118.56 & 64108615 & A & G \\
\hline solcap_snp_sl_47540 & 4 & 4 & 123.75 & 64536101 & $\mathrm{~T}$ & $\mathrm{C}$ \\
\hline solcap_snp_sl_47615 & 4 & 4 & 126.38 & 64829060 & $\mathrm{~T}$ & C \\
\hline solcap_snp_sl_47708 & 4 & 4 & 131.29 & 65351717 & A & C \\
\hline solcap_snp_sl_23608 & 4 & 4 & 134.97 & 66446769 & $\mathrm{C}$ & G \\
\hline solcap_snp_sl_19102 & 5 & 5.1 & 0.00 & 1860363 & $\mathrm{~T}$ & G \\
\hline solcap_snp_sl_5086 & 5 & 5.2 & 0.00 & 7473754 & A & G \\
\hline solcap_snp_sl_1 & 5 & 5.2 & 8.96 & 59889023 & G & A \\
\hline solcap_snp_sl_74 & 5 & 5.2 & 17.04 & 61275800 & $\mathrm{~T}$ & A \\
\hline solcap_snp_sl_75 & 5 & 5.2 & 17.95 & 61500171 & $\mathrm{~T}$ & C \\
\hline solcap_snp_sl_16204 & 5 & 5.2 & 42.02 & 63478118 & $\mathrm{~T}$ & C \\
\hline solcap_snp_sl_25879 & 5 & 5.2 & 49.72 & 64028007 & G & A \\
\hline solcap_snp_sl_37577 & 5 & 5.2 & 51.36 & 64214037 & A & G \\
\hline solcap_snp_sl_37673 & 5 & 5.2 & 58.54 & 64601409 & G & A \\
\hline solcap_snp_sl_354 & 5 & 5.2 & 62.93 & 65071304 & A & G \\
\hline solcap_snp_sl_37825 & 5 & 5.2 & 65.77 & 65269905 & $\mathrm{~T}$ & C \\
\hline solcap_snp_sl_39981 & 6 & 6 & 0.00 & 56871 & A & G \\
\hline solcap_snp_sl_35206 & 6 & 6 & 9.29 & 1748321 & A & G \\
\hline SL10768_412 & 6 & 6 & 28.63 & 33809111 & $\mathrm{C}$ & $\mathrm{T}$ \\
\hline solcap_snp_sl_68935 & 6 & 6 & 32.24 & 36716563 & G & A \\
\hline solcap_snp_sl_55858 & 6 & 6 & 37.25 & 37583093 & A & G \\
\hline solcap_snp_sl_55906 & 6 & 6 & 38.57 & 38008317 & A & $\mathrm{T}$ \\
\hline solcap_snp_sl_24361 & 6 & 6 & 43.65 & 38562515 & A & C \\
\hline solcap_snp_sl_14458 & 6 & 6 & 44.26 & 38851638 & A & G \\
\hline solcap_snp_sl_1337 & 6 & 6 & 49.30 & 39653274 & C & $\mathrm{T}$ \\
\hline solcap_snp_sl_41840 & 6 & 6 & 52.15 & 40448784 & $\mathrm{C}$ & $\mathrm{T}$ \\
\hline solcap_snp_sl_39141 & 6 & 6 & 60.16 & 42492600 & G & A \\
\hline solcap_snp_sl_39246 & 6 & 6 & 61.13 & 42838565 & A & G \\
\hline solcap_snp_sl_57810 & 6 & 6 & 66.90 & 43804153 & $\mathrm{~T}$ & C \\
\hline solcap_snp_sl_57749 & 6 & 6 & 68.98 & 44081669 & $\mathrm{C}$ & $\mathrm{T}$ \\
\hline solcap_snp_sl_57681 & 6 & 6 & 71.35 & 44387315 & $\mathrm{~T}$ & C \\
\hline CL015545-0057_solcap_snp_sl_57521 & 6 & 6 & 74.95 & 45035130 & $\mathrm{~T}$ & G \\
\hline Bcyc_868 & 6 & 6 & 81.03 & 45898856 & G & A \\
\hline solcap_snp_sl_57294 & 6 & 6 & 84.64 & 46293834 & $\mathrm{C}$ & $\mathrm{T}$ \\
\hline solcap_snp_sl_19851 & 6 & 6 & 91.61 & 47400898 & A & G \\
\hline solcap_snp_sl_31676 & 6 & 6 & 94.35 & 48838772 & G & A \\
\hline solcap_snp_sl_11219 & 7 & 7 & 0.00 & 920577 & A & G \\
\hline solcap_snp_sl_68446 & 7 & 7 & 3.50 & 1223861 & A & G \\
\hline solcap_snp_sl_68398 & 7 & 7 & 7.03 & 1557551 & G & A \\
\hline
\end{tabular}




\begin{tabular}{|c|c|c|c|c|c|c|}
\hline Marker name & Chromosome & $\begin{array}{l}\text { Linkage } \\
\text { group }\end{array}$ & $\begin{array}{l}\text { Genetic } \\
\text { Position } \\
\text { (cM) }\end{array}$ & $\begin{array}{c}\text { Physical } \\
\text { position } \\
(\mathrm{Mb})\end{array}$ & $\begin{array}{l}\text { Moneymaker } \\
\text { SNP variant }\end{array}$ & $\begin{array}{l}\text { VG-20 SNP } \\
\text { variant }\end{array}$ \\
\hline solcap_snp_sl_68360 & 7 & 7 & 8.27 & 1697517 & A & G \\
\hline solcap_snp_sl_11170 & 7 & 7 & 11.82 & 1899088 & $\mathrm{~T}$ & C \\
\hline solcap_snp_sl_15791 & 7 & 7 & 17.68 & 2870461 & A & G \\
\hline solcap_snp_sl_22065 & 7 & 7 & 23.55 & 3745280 & A & C \\
\hline solcap_snp_sl_67896 & 7 & 7 & 26.90 & 4210046 & $\mathrm{~T}$ & G \\
\hline solcap_snp_sl_67869 & 7 & 7 & 29.61 & 4605660 & $\mathrm{~T}$ & $\mathrm{C}$ \\
\hline solcap_snp_sl_38868 & 7 & 7 & 36.42 & 56593537 & G & $\mathrm{T}$ \\
\hline $\begin{array}{l}\text { SGN-U577304_snp134_solcap_snp_ } \\
\text { sl_38945 }\end{array}$ & 7 & 7 & 38.27 & 57269711 & G & $\mathrm{C}$ \\
\hline solcap_snp_sl_51773 & 7 & 7 & 41.96 & 58373169 & $\mathrm{~T}$ & G \\
\hline solcap_snp_sl_5853 & 7 & 7 & 49.37 & 59906625 & $\mathrm{~T}$ & C \\
\hline solcap_snp_sl_14163 & 7 & 7 & 54.18 & 60884595 & A & G \\
\hline solcap_snp_sl_19250 & 7 & 7 & 61.28 & 61822692 & $\mathrm{~T}$ & C \\
\hline CL016543-0744_solcap_snp_sl_53433 & 7 & 7 & 64.45 & 62048905 & $\mathrm{~T}$ & $\mathrm{C}$ \\
\hline solcap_snp_sl_53425 & 7 & 7 & 64.45 & 62093144 & G & A \\
\hline solcap_snp_sl_53298 & 7 & 7 & 68.22 & 62687092 & $\mathrm{~T}$ & G \\
\hline solcap_snp_sl_31228 & 7 & 7 & 69.04 & 62921810 & A & G \\
\hline solcap_snp_sl_55505 & 7 & 7 & 77.17 & 63664961 & C & A \\
\hline solcap_snp_sl_31924 & 7 & 7 & 78.19 & 63991821 & $\mathrm{~T}$ & C \\
\hline solcap_snp_sl_71171 & 7 & 7 & 84.77 & 64739981 & A & C \\
\hline solcap_snp_sl_56425 & 8 & 8 & 0.00 & 417491 & G & A \\
\hline solcap_snp_sl_56489 & 8 & 8 & 3.38 & 830226 & G & A \\
\hline solcap_snp_sl_7326 & 8 & 8 & 5.39 & 985676 & C & $\mathrm{T}$ \\
\hline solcap_snp_sl_56575 & 8 & 8 & 8.51 & 1153728 & $\mathrm{C}$ & $\mathrm{T}$ \\
\hline solcap_snp_sl_56631 & 8 & 8 & 17.70 & 1403227 & A & G \\
\hline solcap_snp_sl_14530 & 8 & 8 & 25.11 & 2580721 & A & G \\
\hline solcap_snp_sl_7386 & 8 & 8 & 27.56 & 2849019 & $\mathrm{~T}$ & C \\
\hline solcap_snp_sl_7387 & 8 & 8 & 29.85 & 2908556 & C & $\mathrm{T}$ \\
\hline solcap_snp_sl_4431 & 8 & 8 & 38.81 & 53231057 & C & G \\
\hline solcap_snp_sl_4390 & 8 & 8 & 38.81 & 54457527 & A & G \\
\hline solcap_snp_sl_18196 & 8 & 8 & 44.74 & 56243052 & C & A \\
\hline solcap_snp_sl_29418 & 8 & 8 & 51.11 & 57393693 & $\mathrm{~T}$ & $\mathrm{C}$ \\
\hline solcap_snp_sl_29413 & 8 & 8 & 53.39 & 57576758 & C & $\mathrm{T}$ \\
\hline solcap_snp_sl_34763 & 8 & 8 & 79.17 & 61713636 & $\mathrm{~T}$ & C \\
\hline solcap_snp_sl_15432 & 8 & 8 & 85.08 & 62850886 & $\mathrm{~T}$ & A \\
\hline solcap_snp_sl_15435 & 8 & 8 & 85.08 & 62865675 & A & $\mathrm{T}$ \\
\hline solcap_snp_sl_65262 & 8 & 8 & 87.52 & 63270879 & $\mathrm{~T}$ & C \\
\hline solcap_snp_sl_10181 & 8 & 8 & 91.03 & 64137467 & $\mathrm{~T}$ & A \\
\hline solcap_snp_sl_15471 & 8 & 8 & 91.03 & 64236062 & C & $\mathrm{T}$ \\
\hline solcap_snp_sl_17547 & 9 & 9.1 & 0.00 & 48822 & $\mathrm{~T}$ & G \\
\hline
\end{tabular}




\begin{tabular}{|c|c|c|c|c|c|c|}
\hline Marker name & Chromosome & $\begin{array}{l}\text { Linkage } \\
\text { group }\end{array}$ & $\begin{array}{c}\text { Genetic } \\
\text { Position } \\
(\mathrm{cM})\end{array}$ & $\begin{array}{c}\text { Physical } \\
\text { position } \\
(\mathrm{Mb})\end{array}$ & $\begin{array}{c}\text { Moneymaker } \\
\text { SNP variant }\end{array}$ & $\begin{array}{c}\text { VG-20 SNP } \\
\text { variant }\end{array}$ \\
\hline solcap_snp_sl_39558 & 9 & 9.2 & 0.00 & 4264068 & G & A \\
\hline solcap_snp_sl_39525 & 9 & 9.2 & 4.00 & 4543446 & G & A \\
\hline CLo16894-0326_solcap_snp_sl_39506 & 9 & 9.2 & 5.21 & 4706233 & A & C \\
\hline solcap_snp_sl_39452 & 9 & 9.2 & 6.47 & 4910398 & C & $\mathrm{T}$ \\
\hline solcap_snp_sl_39407 & 9 & 9.2 & 9.09 & 5191470 & G & A \\
\hline solcap_snp_sl_45076 & 9 & 9.2 & 10.78 & 5837005 & A & C \\
\hline solcap_snp_sl_45141 & 9 & 9.2 & 11.62 & 7156689 & G & $\mathrm{T}$ \\
\hline solcap_snp_sl_45162 & 9 & 9.2 & 11.94 & 7855080 & C & $\mathrm{T}$ \\
\hline solcap_snp_sl_43194 & 9 & 9.2 & 16.17 & 62487143 & C & A \\
\hline solcap_snp_sl_43175 & 9 & 9.2 & 16.17 & 62985276 & A & G \\
\hline solcap_snp_sl_43102 & 9 & 9.2 & 18.91 & 63812814 & A & G \\
\hline solcap_snp_sl_49295 & 9 & 9.2 & 24.93 & 65245403 & G & A \\
\hline solcap_snp_sl_29179 & 9 & 9.2 & 35.85 & 66234528 & G & A \\
\hline SGN-U315700_C2_At2g47590_snp377 & 9 & 9.2 & 40.64 & 66958041 & A & G \\
\hline CLo16636-0429 & 9 & 9.2 & 41.95 & 67350015 & $\mathrm{~T}$ & C \\
\hline solcap_snp_sl_29222 & 9 & 9.2 & 43.85 & 67465994 & $\mathrm{~T}$ & C \\
\hline solcap_snp_sl_58222 & 9 & 9.2 & 48.67 & 68371848 & A & G \\
\hline solcap_snp_sl_58253 & 9 & 9.2 & 49.14 & 68604010 & G & A \\
\hline solcap_snp_sl_36856 & 9 & 9.2 & 58.16 & 69819598 & $\mathrm{~T}$ & C \\
\hline solcap_snp_sl_69697 & 9 & 9.2 & 63.91 & 70467811 & G & A \\
\hline solcap_snp_sl_22327 & 9 & 9.2 & 67.00 & 70828802 & $\mathrm{~T}$ & C \\
\hline solcap_snp_sl_36878 & 9 & 9.2 & 69.44 & 71054964 & $\mathrm{C}$ & $\mathrm{T}$ \\
\hline solcap_snp_sl_25745 & 9 & 9.2 & 71.30 & 71185917 & A & C \\
\hline solcap_snp_sl_63588 & 9 & 9.2 & 75.45 & 72380672 & G & A \\
\hline CLo15614-0412 & 10 & 10 & 0.00 & 468921 & C & G \\
\hline solcap_snp_sl_13203 & 10 & 10 & 2.54 & 1175731 & C & $\mathrm{T}$ \\
\hline solcap_snp_sl_17835 & 10 & 10 & 8.08 & 1687857 & A & $\mathrm{T}$ \\
\hline solcap_snp_sl_46445 & 10 & 10 & 13.61 & 2199297 & $\mathrm{~T}$ & C \\
\hline solcap_snp_sl_17859 & 10 & 10 & 15.90 & 2335513 & A & G \\
\hline solcap_snp_sl_30523 & 10 & 10 & 21.22 & 2692905 & A & C \\
\hline SGN-U603133_snp167 & 10 & 10 & 26.46 & 3092679 & $\mathrm{~T}$ & C \\
\hline solcap_snp_sl_9598 & 10 & 10 & 32.07 & 4260136 & G & A \\
\hline solcap_snp_sl_20241 & 10 & 10 & 45.26 & 60561167 & G & A \\
\hline solcap_snp_sl_24679 & 10 & 10 & 49.07 & 61035945 & A & G \\
\hline solcap_snp_sl_33163 & 10 & 10 & 52.87 & 61721457 & $\mathrm{~T}$ & A \\
\hline solcap_snp_sl_33168 & 10 & 10 & 57.23 & 62013257 & $\mathrm{~T}$ & C \\
\hline solcap_snp_sl_61192 & 10 & 10 & 69.58 & 63163119 & $\mathrm{~T}$ & C \\
\hline solcap_snp_sl_24841 & 10 & 10 & 74.63 & 63748200 & A & C \\
\hline $1724 \_1 \_85 b$ & 10 & 10 & 76.25 & 63958041 & G & $\mathrm{T}$ \\
\hline solcap_snp_sl_60643 & 10 & 10 & 91.84 & 65462812 & G & A \\
\hline
\end{tabular}




\begin{tabular}{|c|c|c|c|c|c|c|}
\hline Marker name & Chromosome & $\begin{array}{l}\text { Linkage } \\
\text { group }\end{array}$ & $\begin{array}{c}\text { Genetic } \\
\text { Position } \\
(\mathrm{cM})\end{array}$ & $\begin{array}{c}\text { Physical } \\
\text { position } \\
(\mathrm{Mb})\end{array}$ & $\begin{array}{l}\text { Moneymaker } \\
\text { SNP variant }\end{array}$ & $\begin{array}{l}\text { VG-20 SNP } \\
\text { variant }\end{array}$ \\
\hline solcap_snp_sl_66655 & 11 & 11 & 0.00 & 220859 & $\mathrm{~T}$ & G \\
\hline solcap_snp_sl_66528 & 11 & 11 & 5.59 & 941262 & $\mathrm{~T}$ & G \\
\hline solcap_snp_sl_66462 & 11 & 11 & 7.27 & 1183930 & A & G \\
\hline solcap_snp_sl_66467 & 11 & 11 & 7.27 & 1182112 & $\mathrm{~T}$ & G \\
\hline SGN-U581437_snp1661 & 11 & 11 & 29.11 & 3667512 & $\mathrm{~T}$ & A \\
\hline solcap_snp_sl_21075 & 11 & 11 & 32.56 & 4270887 & A & G \\
\hline $\begin{array}{l}\text { SL1_00sc6004_327317_solcap_snp_ } \\
\text { sl_32056 }\end{array}$ & 11 & 11 & 67.00 & 53407331 & G & A \\
\hline solcap_snp_sl_32030 & 11 & 11 & 67.00 & 53502001 & $\mathrm{~T}$ & A \\
\hline SL1_oosc6004_502854 & 11 & 11 & 67.55 & 53582876 & $\mathrm{~T}$ & A \\
\hline SL1_oosc6004_689170 & 11 & 11 & 68.64 & 53770995 & G & C \\
\hline CLO15534-0232_solcap_snp_sl 56175 & 11 & 11 & 71.54 & 54105654 & A & $\mathrm{C}$ \\
\hline $\begin{array}{l}\text { SL1_00sc6004_1613844_solcap_snp_ } \\
\text { sl_56094 }\end{array}$ & 11 & 11 & 78.01 & 54703558 & G & A \\
\hline solcap_snp_sl_2711 & 11 & 11 & 84.98 & 55071011 & A & G \\
\hline SL1_00sc6004_2273308 & 11 & 11 & 87.42 & 55374896 & $\mathrm{C}$ & $\mathrm{T}$ \\
\hline solcap_snp_sl_17571 & 11 & 11 & 89.02 & 55717679 & A & $\mathrm{C}$ \\
\hline solcap_snp_sl_45010 & 11 & 11 & 91.39 & 56151029 & $\mathrm{~T}$ & C \\
\hline solcap_snp_sl_17717 & 12 & 12.1 & 0.00 & 510496 & $\mathrm{~T}$ & C \\
\hline solcap_snp_sl_13147 & 12 & 12.1 & 4.52 & 714884 & C & $\mathrm{T}$ \\
\hline solcap_snp_sl_33666 & 12 & 12.2 & 0.00 & 6682212 & A & G \\
\hline solcap_snp_sl_17184 & 12 & 12.2 & 16.20 & 60086697 & A & G \\
\hline solcap_snp_sl_3112 & 12 & 12.2 & 9.29 & 36706298 & G & A \\
\hline solcap_snp_sl_31275 & 12 & 12.2 & 28.95 & 62572306 & $\mathrm{~T}$ & $\mathrm{C}$ \\
\hline solcap_snp_sl_42775 & 12 & 12.2 & 29.35 & 62821376 & $\mathrm{~T}$ & $\mathrm{C}$ \\
\hline solcap_snp_sl_55550 & 12 & 12.2 & 34.96 & 63542212 & A & C \\
\hline solcap_snp_sl_31961 & 12 & 12.2 & 38.58 & 63772969 & C & $\mathrm{T}$ \\
\hline solcap_snp_sl_55733 & 12 & 12.2 & 43.07 & 64414152 & $\mathrm{~T}$ & C \\
\hline solcap_snp_sl_32828 & 12 & 12.2 & 51.54 & 65428477 & A & G \\
\hline solcap_snp_sl_32904 & 12 & 12.2 & 52.61 & 65699785 & $\mathrm{C}$ & A \\
\hline solcap_snp_sl_19393 & 12 & 12.2 & 54.13 & 66138091 & G & $\mathrm{T}$ \\
\hline SGN-U603632_snp167 & 12 & 12.2 & 54.13 & 66256781 & A & $\mathrm{T}$ \\
\hline CL009067-0206 & 12 & 12.2 & 56.64 & 66795414 & $\mathrm{~T}$ & C \\
\hline solcap_snp_sl_31342 & 12 & 12.2 & 56.64 & 66885302 & G & $\mathrm{A}$ \\
\hline
\end{tabular}




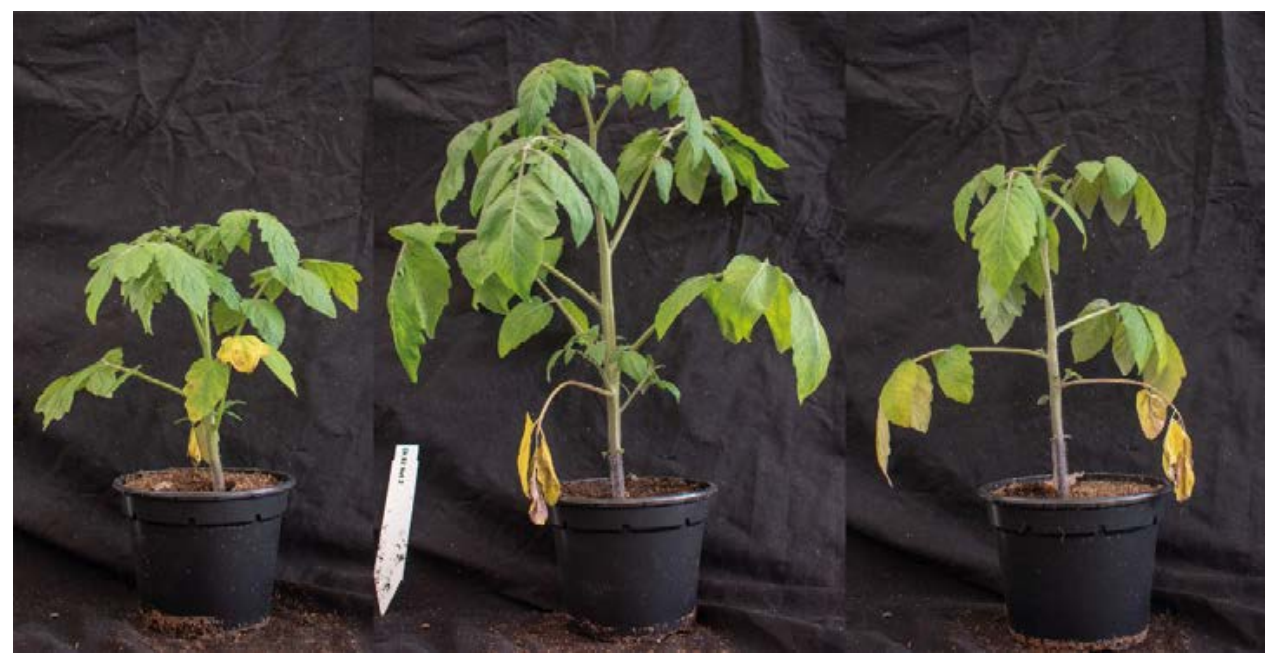

SUPPLEMENTAL FIGURE1 | Example of three RIL genotypes from the Moneymaker x VG-20 RIL population which developed yellowing of the lower leaves. Plants were not inoculated with $V$. dahliae. The picture was taken at 4 weeks after sowing. 


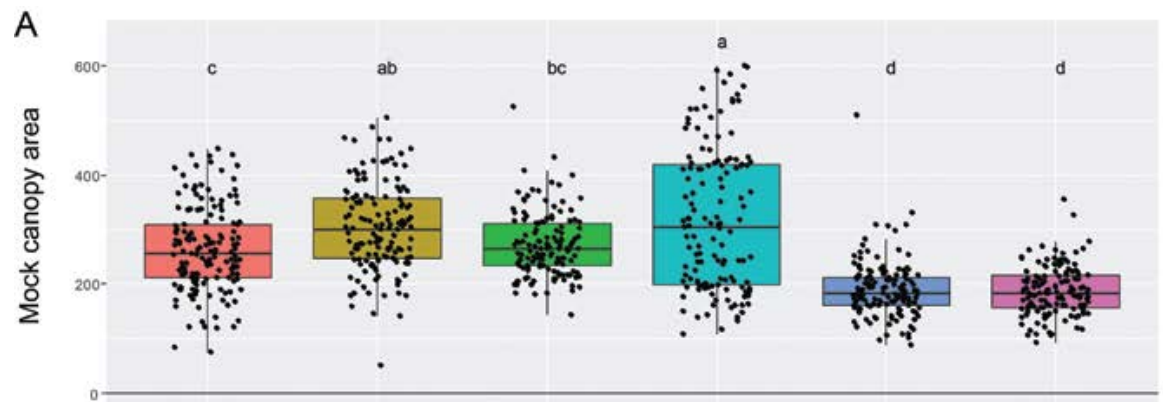

B
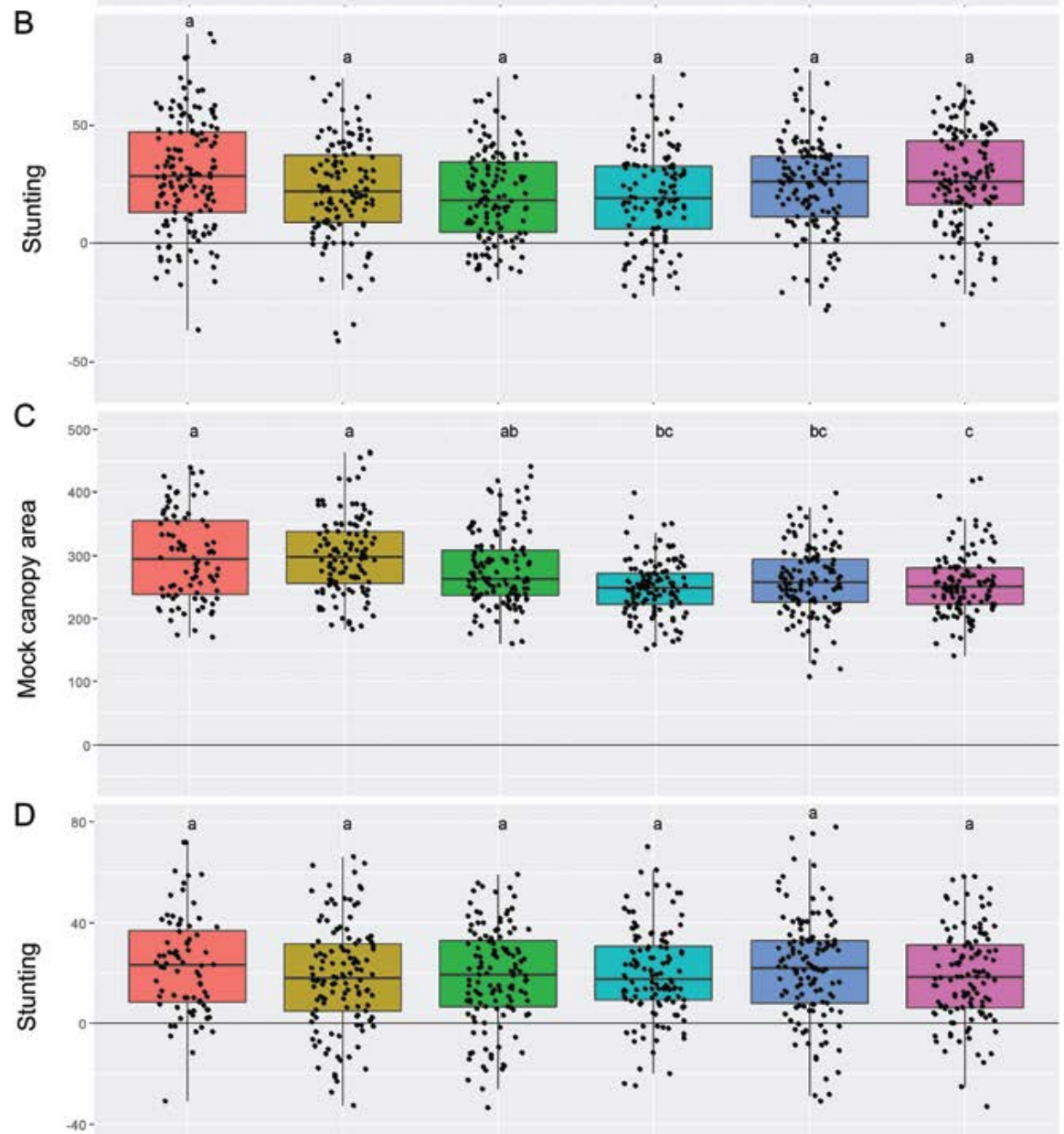

SUPPLEMENTAL FIGURE 2 | Distribution of the canopy area of mock-inoculated plants (A, C) and stunting of $V$. dahliae-inoculated plants (B, D) from the six blocks (x-axis) in the greenhouse. Phenotypes were measured at 24 or 25 dpi. Boxplots with different letter labels are significantly different as determined by a one-way ANOVA, followed by a Fishers' LSD test $(\mathrm{p}=0.01)$ 


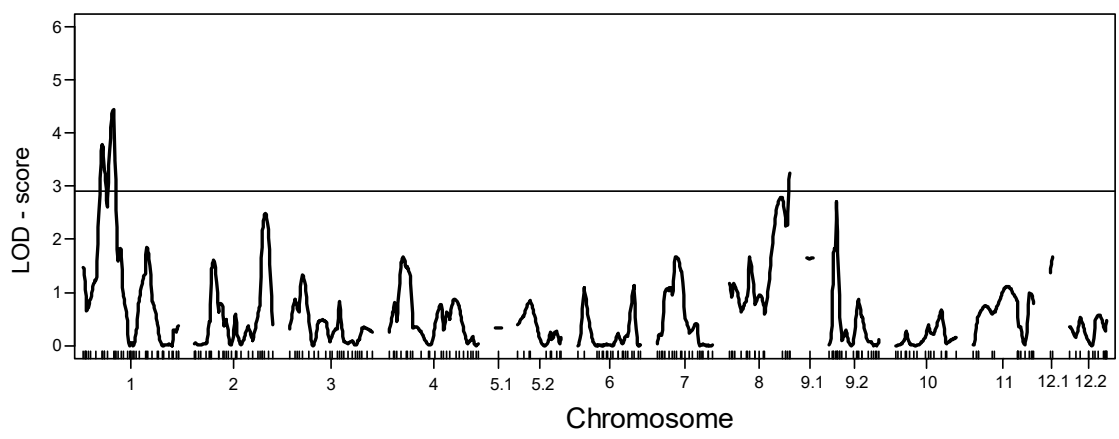

SUPPLEMENTAL FIGURE 3 | Results from an interval mapping analysis for the canopy area of mock-inoculated plants of the Moneymaker x VG-20 RIL population. The horizontal line indicates the significance threshold as determined using a permutation test with 1000 permutations.

SUPPLEMENTAL TABLE 2 | All found indications of QTLs for resistance against V. dahliae DVDS29

\begin{tabular}{|c|c|c|c|c|c|c|c|}
\hline Chromosome & $\begin{array}{l}\text { Position } \\
\text { (cM) }\end{array}$ & $\begin{array}{l}\text { Resistance } \\
\text { donor }\end{array}$ & $\begin{array}{c}\text { Found without } \\
\text { covariates } \\
\text { included? }\end{array}$ & $\begin{array}{l}\text { Found with } \\
\text { covariates } \\
\text { included? }\end{array}$ & $\begin{array}{l}\text { Found with } \\
\text { analysis }\end{array}$ & $\begin{array}{l}\text { Significance } \\
\text { level of QTL } \\
\text { with no covari- } \\
\text { ate included }\end{array}$ & $\begin{array}{c}\text { Significance } \\
\text { level of QTL } \\
\text { with covariate } \\
\text { included }\end{array}$ \\
\hline 1 & 49 & $V G-20$ & yes & no & $\begin{array}{c}\text { Scanone \& } \\
\text { Scantwo }\end{array}$ & $*$ & $\mathrm{n} / \mathrm{a}$ \\
\hline 1 & 143 & VG-20 & no & yes & $\begin{array}{c}\text { Scanone \& } \\
\text { Scantwo }\end{array}$ & $\mathrm{n} / \mathrm{a}$ & $* *$ \\
\hline 3 & 110 & Moneymaker & yes & yes & Scanone & $*$ & $* *$ \\
\hline 6 & 45 & Moneymaker & yes & no & Scantwo & $*$ & $\mathrm{n} / \mathrm{a}$ \\
\hline 6 & 56 & Moneymaker & yes & no & Scantwo & $*$ & $\mathrm{n} / \mathrm{a}$ \\
\hline 7 & 28 & $V G-20$ & no & yes & $\begin{array}{c}\text { Scanone \& } \\
\text { Scantwo }\end{array}$ & $\mathrm{n} / \mathrm{a}$ & $*$ \\
\hline 7 & 54 & Moneymaker & yes & no & $\begin{array}{c}\text { Scanone \& } \\
\text { Scantwo }\end{array}$ & $*$ & $*$ \\
\hline 9.2 & 43 & Moneymaker & yes & yes & Scantwo & * & $*$ \\
\hline 12.2 & 37 & Moneymaker & yes & no & Scantwo & $*$ & $\mathrm{n} / \mathrm{a}$ \\
\hline
\end{tabular}

Indications for QTLs of the Moneymaker x VG-20 RIL population that were found with either the "scanone" or the "scantwo" analysis. Asterisks indicate significance of the QTLs with " $\mathrm{p}<0.05$ and ${ }^{* *} \mathrm{p}<0.01$ determined using permutation tests. 

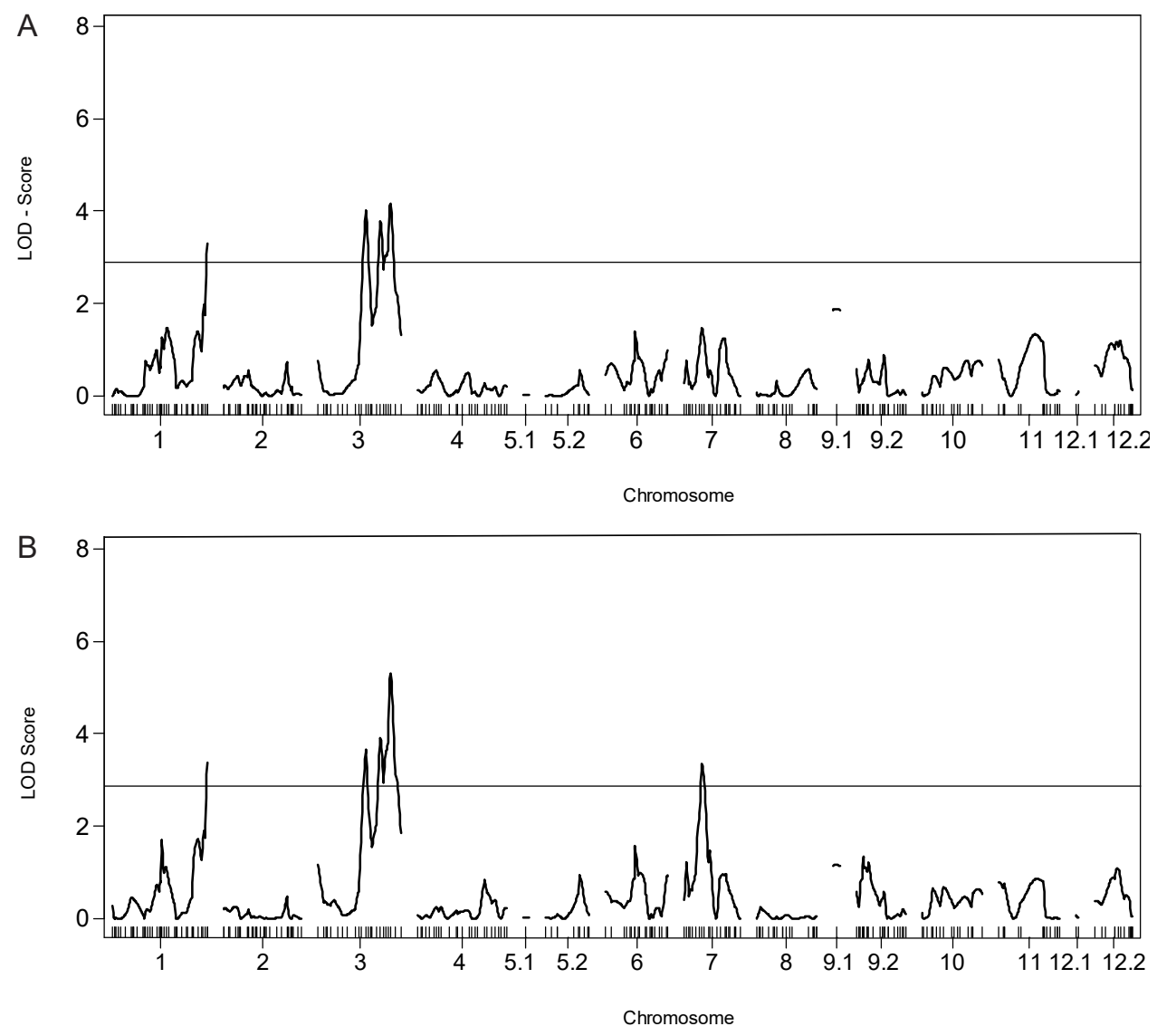

SUPPLEMENTAL FIGURE 4 | A comparison of the results from simple interval mapping without (A) and with (B) mock canopy area and experiment number included as covariates. Horizontal lines indicate the significance threshold as determined with a permutation test with 1000 permutations. 


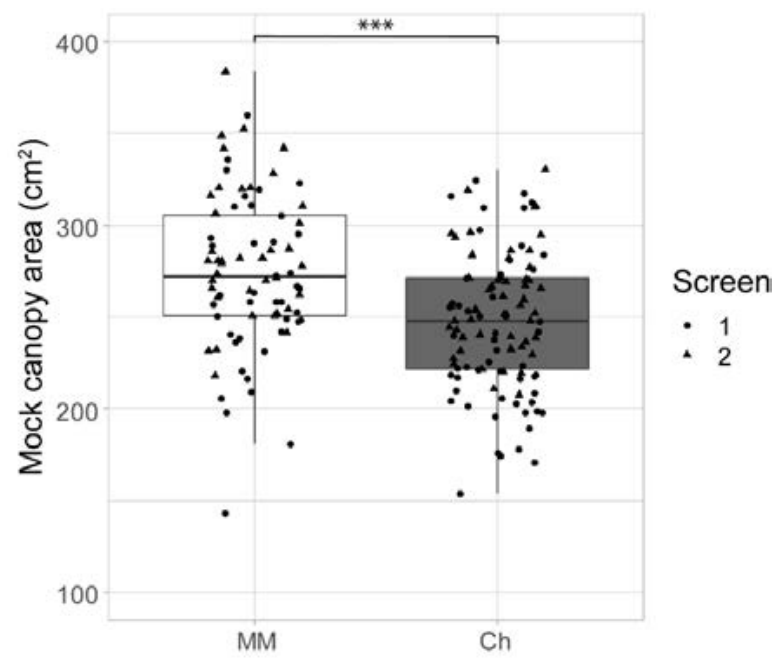

SUPPLEMENTAL FIGURE 5 | Effects of the QTL on Chor at $54 \mathrm{cM}$ for the canopy area of mock-inoculated plants of the Moneymaker (MM) x VG-20 (Ch) RIL population. Asterisks $(* * *)$ indicate significant differences $(\mathrm{p}<0.01)$ using a t-test.

SUPPLEMENTAL TABLE 3 | Comparison of the genetic and physical position of three markers on choi2.

\begin{tabular}{ccccc}
\hline Marker name & $\begin{array}{c}\text { Physical Position } \\
\text { (Mb) SL2.50 }\end{array}$ & $\begin{array}{c}\text { Physical Position } \\
\text { (Mb) SL4.0 }\end{array}$ & $\begin{array}{c}\text { Genetic position (cM) } \\
\text { EXPIMP 2009 } \\
\text { population }\end{array}$ & $\begin{array}{c}\text { Genetic position (cM) } \\
\text { VG-20 population }\end{array}$ \\
\hline solcap_snp_sl_33666 & 6.68 & 6.72 & 65.12 & 39.45 \\
\hline solcap_snp_sl_17184 & 36.71 & 60.85 & 75.41 & 42.02 \\
\hline solcap_snp_sl_3112 & 60.09 & 37.40 & 68.14 & 41.76 \\
\hline
\end{tabular}

Physical positions of the markers were determined based on the tomato reference assembly from $S$. lycopersicum 'Heinz', assembly version SL2.5 and SL4.0

SUPPLEMENTAL TABLE 4 | Results of a one-way ANOVA with stunting as explained variate and experiment number and canopy area of the mock-inoculated plants as a co-factor.

\begin{tabular}{lccccc}
\hline Factor & Df & Sum Sq & Mean Sq & F value & Pr(>F) \\
\hline Experiment number & 1 & 534.2 & 534.2 & 4.9345 & 0.0275 \\
\hdashline Area & 1 & 2511.8 & 2511.77 & 23.2016 & $2.96 \mathrm{E}-06$ \\
\hdashline Residuals & 191 & 20677.4 & 108.26 & & \\
\hline
\end{tabular}

SUPPLEMENTAL TABLE 5 | LOD scores from the two-dimensional, two QTL scan "scantwo" without the inclusion of covariates. Asterisks indicate significant LOD-scores $\left(^{* *}=\mathrm{p}<0.01,{ }^{*}=\mathrm{p}<0.05\right)$. QTL analysis was performed in $\mathrm{R}$ with the package R/QTL. 


\begin{tabular}{cccccc}
\hline QTL-pair & $\begin{array}{c}\text { Position } \\
\text { QTL 1 (cM) }\end{array}$ & $\begin{array}{c}\text { Position } \\
\text { QTL 2 (cM) }\end{array}$ & $\begin{array}{c}\text { LOD score for >1 } \\
\text { QTL, allowing for } \\
\text { interactions }\end{array}$ & $\begin{array}{c}\text { LOD score for an } \\
\text { interaction between } \\
\text { QTLs }\end{array}$ & $\begin{array}{c}\text { LOD score for 2 } \\
\text { additive QTLs }\end{array}$ \\
\hline Chr 1: Chr 12.2 & 49 & 37 & $4.91^{*}$ & 3.85 & 1.06 \\
\hdashline Chr 6: Chr 6 & 45 & 56 & $4.76^{*}$ & 2.04 & 2.72 \\
\hdashline Chr 7: $\mathrm{Chr} 9.2$ & 28 & 43 & $5.34^{*}$ & 3.83 & 1.51 \\
\hline
\end{tabular}

SUPPLEMENTAL TABLE 6 | LOD scores from the two-dimensional, two QTL scan "scantwo" with the inclusion of covariates. Asterisks indicate significant LOD-scores $\left(^{* *}=\mathrm{p}<0.01,{ }^{*}=\mathrm{p}<0.05\right)$. QTL analysis was performed in $\mathrm{R}$ with the package R/QTL.

\begin{tabular}{cccccc}
\hline QTL-pair & $\begin{array}{c}\text { Position } \\
\text { QTL 1 (cM) }\end{array}$ & $\begin{array}{c}\text { Position } \\
\text { QTL 2 (cM) }\end{array}$ & $\begin{array}{c}\text { LOD score for >1 } \\
\text { QTL, allowing for } \\
\text { interactions }\end{array}$ & $\begin{array}{c}\text { LOD score for an } \\
\text { interaction between } \\
\text { QTLs }\end{array}$ & $\begin{array}{c}\text { LOD score for 2 } \\
\text { additive QTLs }\end{array}$ \\
\hline Chr 1: Chr 7 & 143 & 28 & $5.64^{* *}$ & 1.12 & $4.53^{* *}$ \\
\hline Chr 7: Chr 7 & 29 & 54 & $4.95^{*}$ & 1.25 & $3.71^{*}$ \\
\hdashline Chr 7: Chr 9.2 & 27 & 11 & $5.35^{*}$ & 2.51 & 2.84 \\
\hline
\end{tabular}

A
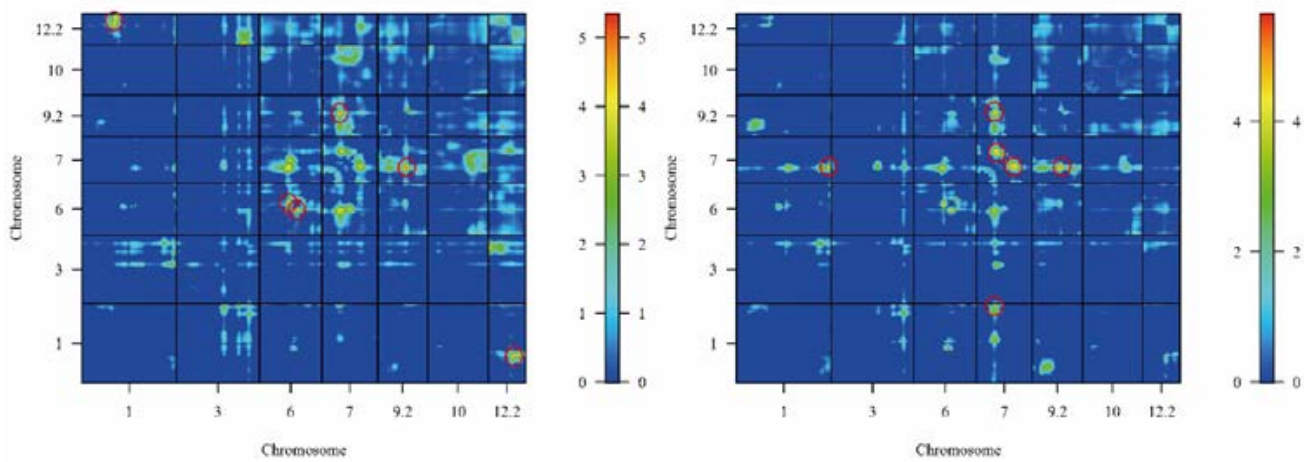

SUPPLEMENTAL FIGURE 6 | LOD scores from the $2 \mathrm{D}$ - two QTL scan analysis without covariate (A) and with mock canopy area and experiment included as covariates (B). Red circles highlight positions with significant LOD scores $(\mathrm{p}<0.05)$ as determined using a two-dimensional permutation test with 1000 permutations. LOD scores indicate the likelihood of a pair of two QTLs over the likelihood of a single QTL, allowing for epistatic interactions. QTL analysis was performed in $\mathrm{R}$ with the package R/QTL. 


\title{
Chapter 6
}

\section{Comparative genomics reveals two candidates for the Verticillium dahliae in planta-secreted effector protein recognized in $\mathbf{2}$ tomato plants}

\author{
Edgar A. Chavarro-Carrero",", \\ Jasper P. Vermeulen ${ }^{1,2,}$, \\ Toshiyuki Usami ${ }^{3}$, \\ Henk J.Schouten², \\ Yuling Bai ${ }^{2}$, \\ Michael F. Seidl ${ }^{1,4}$ and \\ Bart P.H.J. Thomma ${ }^{1,5}$
}

'Laboratory of Phytopathology, Wageningen University \& Research, 6708 PB Wageningen, The Netherlands ${ }^{2}$ Laboratory of Plant Breeding, Wageningen University \& Research, 6708 PB Wageningen, The Netherlands. ${ }^{3}$ Craduate School of Horticulture, Chiba University, Matsudo, Chiba 271-8510, Japan.

${ }^{4}$ Theoretical Biology \& Bioinformatics Group, Department of Biology, Utrecht University, Utrecht, The Netherlands. ${ }^{5}$ Cluster of Excellence on Plant Sciences (CEPLAS), University of Cologne, Botanical Institute, Cologne, Germany. These authors contributed equally to this work 


\section{Abstract}

Plant pathogens secrete effector molecules during host invasion to promote host colonization. However, some of these effectors are recognized by host receptors to activate an immune response. Recently, resistance in tomato against strains of the soilborne vascular wilt fungus Verticillium dahliae that belong to race 2, mediated by the single dominant V2 locus, was identified. In this study, we performed comparative genomics between race 2 strains, that are contained by V2 resistance, and V2 resistance-overcoming race 3 strains to identify the avirulence effector that activates the V2 resistance. Based on pathogenicity profiling on $V 2$ resistant tomato plants, a collection of $V$. dahliae isolates was categorized into race 2 and race 3 strains. Ultimately, we identified $277 \mathrm{~kb}$ race 2 -specific sequences comprising only two genes that encode predicted secreted proteins, and both are expressed during $V$. dahliae colonization of tomato plants. These two effector genes reside in close proximity of each other and, consequently, no natural isolates of $V$. dahliae could be found that possess only one of the two genes. Future functional analysis based on deletion of individual genes from race 2 isolates and introduction into race 3 isolates, will have to reveal which of the two candidates is the genuine avirulence effector gene that corresponds to tomato $\mathrm{V} 2$. 


\section{Introduction}

In nature, plants are continuously threatened by potential plant pathogens. However, most plants are resistant to most potential plant pathogens due to passive and inducible defence mechanisms, including preformed physical barriers, chemical defences and immune responses that are activated by invasion patterns of diverse nature (Cook, et al., 2015; Dangl \& Jones, 2001). Throughout time, different conceptual frameworks have been put forward to describe the molecular basis of plant-pathogen interactions and the mechanistic underpinning of plant immunity. Initially, Harold Flor introduced the gene-for-gene model in which the product of a single dominant host gene, termed a resistance $(R)$ gene, induces a resistance phenotype in response to a pathogen expressing a single dominant avirulence (Avr) gene (Flor 1942). Isolates of the pathogen which lost the avirulence gene or which possess a variant of which the product is not recognized by the $R$ gene escape recognition and are assigned to a resistance-overcoming race. In parallel to these race-specific Avrs, non-racespecific elicitors were described as conserved microbial molecules that are often recognized by multiple plant species (Darvill and Albersheim 1984). The recognition by plants both of Avrs and of non-race-specific elicitors, presently known as microbe-associated molecular patterns (MAMPs), was combined in the 'zig-zag' model (Jones and Dangl 2006). In this model, MAMPs are perceived by cell surface-localized pattern recognition receptors (PRRs) to trigger MAMP-triggered immunity (MTI), while effectors are recognized by cytoplasmic receptors that are known as resistance $(\mathrm{R})$ proteins to activate effector-triggered immunity (ETI) (Jones and Dangl 2006). Importantly, the model recognizes that Avrs function to suppress host immune responses in the first place, implying that these molecules, besides acting as avirulence determinants, act as virulence factors through their function as effector molecules (Jones and Dangl 2006). It is presently recognized that microbial pathogens secrete dozens to hundreds of effectors in their hosts to contribute to the establishment of disease, only some of which become recognized and act as Avr (Rovenich et al., 2014). A more recent model, termed the Invasion Model, recognizes that the functional separation of MTI and ETI is problematic for several reasons and proposes that the corresponding receptors, collectively termed invasion pattern receptors (IPRs), detect either externally encoded or modified-self ligands that indicate invasion, termed invasion patterns (IPs), to mount an effective immune response (Cook et al., 2015; Thomma et al., 2011). After IPR activation, a wide array of cellular events take place, including a rapid influx of calcium ions, a burst of active oxygen species release, activation of mitogen-activated protein kinases (MAPKs), reprogramming of gene expression, deposition of callose cell wall appositions at sites of attempted infection and, often, a localized cell death that is called the hypersensitive response (HR) (Jones and Dangl 2006).

Plant invasion pattern receptors (IPR) encompass typical $R$ genes which have been exploited for almost a century to confer resistance against plant pathogens upon introgression wild 
relatives into elite cultivars (Dangl et al., 2013; Dodds \& Rathjen, 2010). Most $R$ genes encode members of a highly polymorphic superfamily of intracellular nucleotide-binding leucinerich repeat (NLR) receptors, while some others encode cell surface receptors (Dangl et al. 2013). Whereas some $\mathrm{R}$ proteins directly interact with their corresponding avirulence (Avr) effectors to activate immunity, others monitor the manipulation of guarded cellular host proteins by pathogen avirulence (Avr) effectors in order to mount defence (van der Biezen and Jones 1998; Jones and Dangl 2006). Unfortunately, most $R$ genes used in commercial crops are short-lived in the field because they are overcome by pathogen populations as their deployment in monoculture-based crop systems selects for pathogen variants that have overcome the $R$ gene-based immunity (Stukenbrock and McDonald 2008; Dangl et al. 2013). Overcoming of resistance occurs upon purging of the Avr gene, sequence diversification such that the gene product is no longer recognized, or by subversion of host immunity that is activated by the Avr through other effectors (Cook et al. 2015). For example, for the tomato leaf mould fungus Cladosporium fulvum that has been studied as a model to understand the gene-for-gene hypothesis for decades, loss of resistance mediated by a suite of $R$ genes has been attributed to allelic variation of Avrs. This allelic variation ranges from single nucleotide polymorphisms, leading to highly unstable effector proteins and loss of recognition, through gene inactivation by means of the insertion of transposonlike elements, to loss of the complete Avr gene (Stergiopoulos et al., 2007).

The molecular cloning of the first bacterial Avr gene from Pseudomonas syringae pv. glycinea was reported in 1984 (Staskawicz et al., 1984), the first fungal Avr gene from Cladosporium fulvum in 1991 (van Kan et al., 1991) and the first oomycete Avr gene from Phytophthora sojae in 2004 (Shan et al., 2004). Dozens of additional Avrgenes have been cloned since then. Initially, such Avr genes were identified by reverse genetics and map-based cloning strategies, but also other approaches have been exploited such as functional screens in which candidate libraries were tested on plants that express the corresponding $R$ gene (Takken et al. 2000; Mesarich et al. 2014). More recently, the availability of genome sequences due to declining sequencing costs has allowed the cloning of novel Avrs by combining comparative genomics or transcriptomics with functional assays (de Jonge et al., 2012; Mesarich et al., 2014).

Verticillium wilt is a vascular wilt disease that is caused by the soil-borne fungal pathogen Verticillium dahliae that has a broad host-range comprising of hundreds dicotyledonous plant species, including numerous crops such as tomato, potato, lettuce, olive and cotton (Fradin \& Thomma, 2006; Klosterman et al., 2009). The first source of genetic resistance against Verticillium wilt was identified in tomato in the early 1930s in an accession called Peru Wild (Schaible et al., 1951). The resistance is governed by a single dominant locus, designated $V e$ (Diwan et al., 1999), comprising two genes that encode cell surface receptors of which one, Ve1, acts as a genuine resistance gene (Fradin and Thomma 2006). Shortly after its deployment in the 1950s, resistance-overcoming strains have appeared that were 
assigned to race 2 whereas strains that are contained by VeI-resistance belong to race 1 (Alexander 1962). Thus, Ve1 is characterized as a race-specific $R$ gene, and resistanceovercoming strains have become increasingly problematic over time (Alexander, 1962; Dobinson et al., 1996). With comparative population genomics of race 1 and race 2 strains, the $V$. dahliae avirulence effector that is recognized by tomato Ve1 was identified as VdAve1, a secreted effector that is induced during host colonization (de Jonge et al., 2012). As anticipated, it was demonstrated that VdAver acts as a virulence factor on tomato plants that lack the functional Ver gene and that, consequently, cannot recognize VdAver (de Jonge et al., 2012). Whereas all race 1 strains carry an identical copy of $V d A v e 1$, all race 2 strains analysed to date are characterized by complete loss of the VdAver locus (de Jonge et al., 2012; Faino et al., 2016). Intriguingly, phylogenetic analysis has revealed that VdAver was horizontally acquired by $V$. dahliae from plants (de Jonge et al., 2012), after which the effector gene was lost multiple times independently, presumably due to selection pressure exerted by the Ve1 locus that has been introgressed in most tomato cultivars (Faino et al. 2016). Despite significant efforts, attempts to identify tomato resistance sources against $V$. dahliae race 2 strains have remained unsuccessful for a long time (Baergen et al., 1993). Recently, however, a source of race 2 resistance was identified in the wild tomato species Solanum neorickii (formerly Lycopersicon parviflorum) (Usami et al. 2017). This genetic material was subsequently used to develop the rootstock tomato cultivars Aibou, Ganbarune-Karis and Back Attack by Japanese breeding companies, in which resistance is controlled by a single dominant locus, denoted V2 (Usami et al. 2017). However, experimental trials using race 2-resistant rootstocks revealed Verticillium wilt disease in some of the plots, pointing towards the existence of $V 2$ resistance-overcoming $V$. dahliae strains that, consequently, are assigned to race 3 (Usami et al. 2017). In this study, we performed comparative genomics based on Oxford Nanopore sequencing technology, combined with functional assays, to identify the avirulence effector that activates race-specific resistance in tomato lines that carry V2.

\section{Results}

\section{Identification of Verticillium dahliae strains that escaped $\mathbf{V} 2$ recognition in tomato}

Recently, based on the single dominant $V 2$ locus, race 2-resistant tomato rootstock cultivars were developed that include the rootstock cultivar Aibou. While testing the efficacy of the resistance, $V 2$ resistance-overcoming strains of $V$. dahliae were found that belong to race 3 (Usami et al. 2017). To identify the $V$. dahliae avirulence gene encoding the effector that is recognized by tomato $V 2$, we pursued a comparative genomics strategy with the aim to identify race 2 -specific regions that are present in every race 2 strain and absent in every race 3 strain. We hypothesized that these race 2 -specifc regions comprise the Avr gene 
encoding the effector that is recognized by V2. To this end, pathogenicity assays were performed with a collection of $V$. dahliae strains on a differential set of tomato genotypes, comprising (I) Moneymaker plants that are susceptible to all $V$. dahliae races, (II) VeItransgenic Moneymaker plants that are resistant to race 1 but not to race 2 strains of $V$. dahliae (Fradin et al. 2009), and (III) Aibou plants that carry Ve1 and V2 and are therefore resistant to race 1 as well as race 2 strains of the pathogen (Usami et al. 2017). First, we aimed to confirm the race assignment of eight $V$. dahliae strains that were previously tested by Usami et al. (2017). Additionally, three strains that were previously assigned to race 2 were included in our study (de Jonge et al., 2012). Arguably, V. dahliae genome assemblies of the highest quality are the previously generated gapless telomere-to-telomere assemblies of strains VdLs17 and JR2 isolated from lettuce and tomato, respectively (Faino et al., 2015). As $V$. dahliae strain VdLs17 is weakly pathogenic on tomato, we attempted to make use of the race 1 JR2 strain for our comparative genomics strategy.

At three weeks post inoculation, all strains were found to cause significant stunting on the susceptible control Moneymaker (Fig. 1A), confirming that all strains have the basic ability to cause disease on tomato. Similarly, all strains, except for the race 1 strain JR2, caused significant stunting on race 1-resistant Ve1-transgenic Moneymaker plants (Fig. 1B), confirming that these strains lack the VdAve1 effector that is recognized by Ve1. This observation implies that, except for strain JR2, none of the strains is of race 1 and that a possible containment on Aibou plants cannot be caused by Ve1 recognition of the VdAve1 effector. Importantly, we confirmed that most of the strains that were used by Usami et al. (2017) and that were previously assigned to race 2 did not cause significant stunting on Aibou, whereas most of the strains that were assigned to race 3 caused clear stunting symptoms (Fig. 1C). More specifically, this concerned the race 2 strains TO22, UD-1-4-1 and GF-CA2 and the race 3 strains HOMCF, GF-CB5 and GF1192 (Fig. 1; Usami et al., 2017). The exceptions are strains GF1207 and VT-2A that were previously assigned to race 2 and 3, respectively (Usami et al. 2017), but for which the phenotyping in our assays was not unambiguous due to a relatively low degree of virulence on Moneymaker plants (Fig. IA, C). The previously assigned race 2 strain DVDS26 (de Jonge et al., 2012) caused no significant stunting on Aibou plants, confirming that this remains to be a race 2 strain, while strains DVD161 and DVD3 caused significant stunting, implying that these strains should be assigned to race 3. As expected, the race 1 strain JR2 did not cause stunting on Aibou plants, which can at least partially be attributed to Aver effector recognition by the Ver gene product in these plants. However, the finding that a transgenic line of the JR2 strain from which the gene that encodes the race 1-specific effector Ave1 was deleted (JR2 $\Delta$ Ave1; de Jonge et al., 2012) caused significant stunting on Ve1-transgenic Moneymaker and not on Aibou plants, suggests that the JR2 strain carries the gene that encodes the avirulence molecule that is recognized by the $V 2$ gene product. However, this interpretation needs to be made with caution given that the aggressiveness of the VdAver deletion strain on tomato is severely 
reduced (de Jonge et al., 2012), as can also be observed on Moneymaker plants in our assays (Fig. 1A). This observation, combined with the observation that stunting on Aibou plants by any race 3 strain is generally less than stunting on Moneymaker plants (Fig $1 A-C$ ), could indicate that basal defence against Verticillium wilt is enhanced in Aibou plants, and thus that incompatibility of the Aver deletion strain may be due to enhanced basal defence of Aibou plants rather than due to V2-mediated recognition of the corresponding avirulence molecule in the JR2 strain.
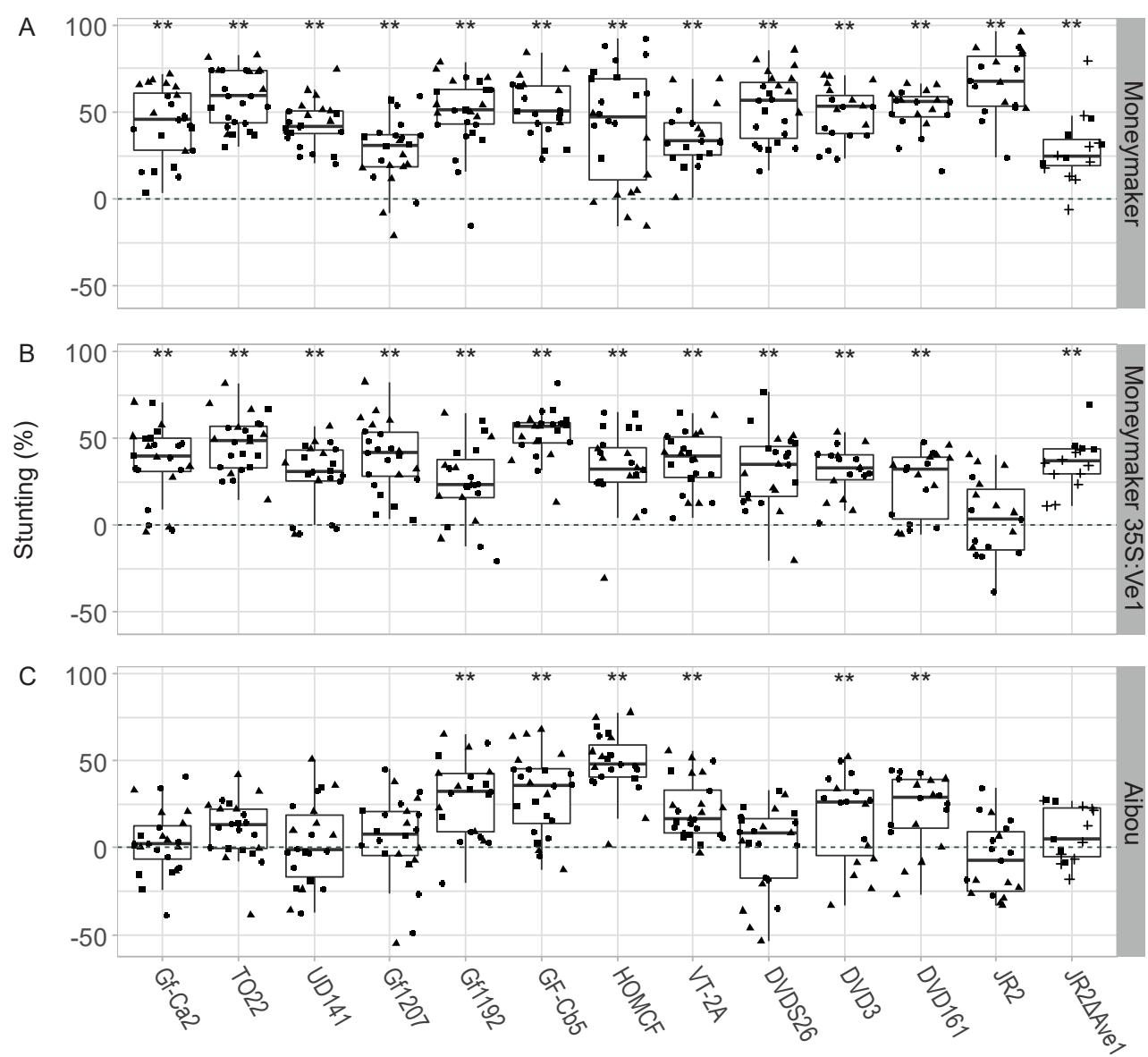

FICURE 1 | Pathogenicity phenotyping of a collection of Verticillium dahliae strains on tomato. V. dahliae-induced stunting of wild-type Moneymaker plants (A), Ve1-transgenic Moneymaker plants (35S:Ve1) (B) and Aibou plants (C) as measured at 21 days post inoculation. The graphs show collective data from four different experiments indicated with different symbols (circles, squares, triangles and plus symbols), and asterisks indicate significant stunting as determined with an ANOVA followed by a Fishers' LSD test $(\mathrm{p}<0.01)$. 


\section{Comparative genomics to identify Verticillium dahliae Av2 candidates}

Based on our phenotyping results, showing that unambiguous race assignment was not possible for every strain, four different groupings into race 2 and 3 strains were made with different degrees of stringency for the set of twelve $V$. dahliae strains (Table 1 ). The clearest phenotyping results in our assays were obtained for the strains that were previously phenotyped, i.e. the race 2 strains TO22, UD-1-4-1 and GF-CA2 and the race 3 strains HOMCF, GF-CB5 and GF1192 (Usami et al. 2017). Thus, for the most stringent approach (I) comparative genomics was performed based only on these six strains (Table 2). Furthermore, our phenotyping assays consistently assigned the strain DVDS26 as race 2, and strains DVD161 and DVD3 as race 3. Therefore, in a second approach (II), the strains of approach I were supplemented with these three strains (Table 2). For approach III, strains of approach II were supplemented with the previously assigned race 2 strain GF1207 and the previously assigned race 3 strain VT-2A (Usami et al. 2017), for which the phenotyping was not unambiguous in our assays (Table 2). Finally, in approach IV we used the JR2 genome assembly (Faino et al., 2015) as a reference for comparative genomics with the selection of race 2 and race 3 strains as defined in approach II (Table 2).

Besides a previously generated gapless telomere-to-telomere assembly of strain JR2 (Faino et al., 2015), genome assemblies were also available for strains DVDS26, DVD161 and DVD3, albeit that these assemblies were highly fragmented as these were based on Illumina sequencing (David Torres, unpublished data) (Table 1). In this study, we determined the genome sequences of four additional $V$. dahliae strains that belong to race 2, namely $\mathrm{TO} 22$, UD1-4-1, GF1207 and GFCA2, and four additional race 3 strains, namely GFCB5, GF1192, VT2A and HOMCF, with Oxford Nanopore sequencing Technology (ONT) using a MinION device (Table 1). For each strain, $\sim 2-3 \mathrm{~Gb}$ of sequence data was produced, representing 50$100 x$ genome coverage based on the $\sim 35 \mathrm{Mb}$ gapless reference genome of $V$. dahliae strain JR2 (Faino et al. 2015). Subsequently, self-correction of the reads, read trimming and genome assembly was performed, leading to genome assemblies ranging from 18 contigs for strain UD1-4-1 to 69 for strain GF1207, respectively (Table 1).

To perform comparative genomics, for each of the approaches self-corrected reads from the selected $V$. dahliae race 3 strains were mapped against the designated reference genome and regions that were not covered by race 3 reads were retained. For approaches I-III the assembly of $V$. dahliae strain TO22 was used as a reference, whereas for approach IV the telomere-to-telomere assembly of strain JR2 (Faino et al., 2015) was used (Table 1). Next, self-corrected reads from the selected race 2 strains were mapped against the retained reference genome-specific regions that are absent from the race 3 strains. Sequences that were found in every race 2 strain were retained as candidate regions to encode the Avr effector. Sequences that are shared by the $V$. dahliae strain TO22 reference assembly and the race 2 strains, and that are absent from the race 3 strains, were mapped against 
the $V$. dahliae strain JR2 genome assembly, and common genes were extracted. Sequences that did not map to the $V$. dahliae strain JR2 genome assembly were annotated de novo using Augustus.

Our strategy revealed race 2-specific regions of a total length of $670 \mathrm{~kb}$, containing 122 genes of which eight encode putative secreted proteins for approach I with the most stringent selection of strains to be included in the analysis (Table 2). For approach II, the addition of three strains to those included in approach I reduced the race 2 -specific regions to $660 \mathrm{~kb}$, containing 115 genes, of which six encode putative secreted proteins (Table 2). For approach III, the further addition of two strains for which the phenotyping was somewhat ambiguous slightly reduced the amount of candidate regions to $563 \mathrm{~kb}$ comprising 110 genes. However, the same six putatively secreted proteins remained as candidates, so effectively the list of Avr candidates remained the same as for approach II (Table 2). The last comparison in approach IV, based on the reference assembly of the $V$. dahliae JR2 strain, identified a $277 \mathrm{~kb}$ race 2 -specific region with only two genes that are predicted to encode secreted proteins; the previously identified proteins XLOC_00170 (currently named VDAG_JR2_Chr4g03680a) and evm.model.contig1569.344 (currently named VDAG_JR2_Chr4g03650a), further referred to as Evm_344 (de Jonge et al. 2013; Faino et al., 2015).

TABLE $1 \mid$ V. dahliae strains used in this study for comparative genomics and genome assembly statistics.

\begin{tabular}{|c|c|c|c|c|c|c|c|c|c|c|}
\hline Strain & $\begin{array}{l}\text { old } \\
\text { race }\end{array}$ & Ref. ${ }^{1}$ & $\begin{array}{l}\text { New } \\
\text { race }\end{array}$ & Platform $^{2}$ & $\begin{array}{l}\text { Data } \\
(\mathrm{Gb})^{3}\end{array}$ & $\begin{array}{l}\text { Assembly } \\
\text { size }(\mathrm{Mb})\end{array}$ & $\begin{array}{l}\text { No. of } \\
\text { contigs }^{4}\end{array}$ & $\begin{array}{l}\text { Contig } \\
\text { N50 (Kb) }\end{array}$ & $\begin{array}{l}\text { Used in } \\
\text { approaches }\end{array}$ & Ref. $^{1}$ \\
\hline $\mathrm{JR2}$ & 1 & A & 1 & PacBio & 8.9 & 36.1 & 8 & 4168 & IV & $\mathrm{D}$ \\
\hline DVDS26 & 2 & $B$ & 2 & Illumina & 1 & 35.3 & 5,361 & $47.1^{*}$ & II, III, IV & $\mathrm{B}$ \\
\hline DVD161 & 2 & B & 2,3 & Illumina & 1 & 34.1 & 4,078 & $42.4^{*}$ & II, III, IV & B \\
\hline DVD3 & 2 & $B$ & 2,3 & Illumina & 1 & 34.1 & 9,318 & $43.9^{*}$ & II, III, IV & $B$ \\
\hline $\mathrm{TO} 22$ & 2 & C & 2 & Nanopore & 4 & 34.9 & 20 & 12.4 & I, II, III, IV & TS \\
\hline UD1-4-1 & 2 & $\mathrm{C}$ & 2 & Nanopore & 1.9 & 34.6 & 18 & 18.1 & I, II, III, IV & TS \\
\hline GF1207 & 2 & $\mathrm{C}$ & 2 & Nanopore & 1.6 & 34.8 & 69 & 8.5 & III & TS \\
\hline GF-CA2 & 2 & $\mathrm{C}$ & 2 & Nanopore & 2 & 35 & 38 & 9.1 & I, II, III, IV & TS \\
\hline GF-CB5 & 3 & $\mathrm{C}$ & 2,3 & Nanopore & 4 & 34.8 & 19 & 11.4 & I, II, III, IV & TS \\
\hline VT-2A & 3 & $\mathrm{C}$ & 2,3 & Nanopore & 1.8 & 34.8 & 22 & 10.2 & III & TS \\
\hline GF1192 & 3 & $\mathrm{C}$ & 2,3 & Nanopore & 2 & 34.6 & 23 & 14.5 & I, II, III, IV & TS \\
\hline HOMCF & 3 & $\mathrm{C}$ & 2,3 & Nanopore & 2 & 36.1 & 33 & 10.1 & I, II, III, IV & TS \\
\hline
\end{tabular}

${ }^{1}$ References: A: Fradin et al., 2009; B: de Jonge et al., 2012; C: Usami et al., 2017; D: Faino et al., 2015; TS: this study. ${ }^{2}$ Sequencing platform used.

${ }^{3}$ Amount of sequencing data generated

${ }^{4}$ For strains 
TABLE 2 | Comparative genomics for four different groupings of race 2 and race 3 strains to reveal race 2 -specific Avr candidates.

\begin{tabular}{|c|c|c|c|c|}
\hline Approach & I & II & III & IV \\
\hline Race 2 reference & $\mathrm{TO} 22$ & $\mathrm{TO} 22$ & $\mathrm{TO} 22$ & $\mathrm{JR} 2$ \\
\hline \multirow[t]{4}{*}{ Race 2} & GF-CA2 & GF-CA2 & GF-CA2 & GF-CA2 \\
\hline & $\mathrm{TO} 22$ & $\mathrm{TO} 22$ & $\mathrm{TO} 22$ & $\mathrm{TO} 22$ \\
\hline & UD-1-4-1 & UD-1-4-1 & UD-1-4-1 & UD-1-4-1 \\
\hline & & DVDS26 & DVDS26 GF1207 & DVDS26 \\
\hline \multirow[t]{6}{*}{ Race 3} & GF-1192 & GF-1192 & GF-1192 & GF-1192 \\
\hline & GF-CB5 & GF-CB5 & $\mathrm{GF}-\mathrm{CB} 5$ & $\mathrm{GF}-\mathrm{CB} 5$ \\
\hline & HOMCF & HOMCF & HOMCF & HOMCF \\
\hline & & DVD161 & DVD161 & DVD161 \\
\hline & & $\mathrm{DVD}_{3}$ & DVD3 & DVD3 \\
\hline & & & VT-2A & \\
\hline Retained (kb) & 670 & 660 & 563 & 277 \\
\hline Shared with JR2 (kb) & 282 & 277 & 222 & 277 \\
\hline \#JR2 genes & 44 & 41 & 40 & 41 \\
\hline \#Augustus genes & 78 & 74 & 70 & -- \\
\hline \#Secreted & 8 & 6 & 6 & 2 \\
\hline \multirow[t]{9}{*}{ Retained candidates } & XLOC_00170 & XLOC_00170 & XLOC_00170 & XLOC_00170 \\
\hline & $\begin{array}{c}\text { evm.model. } \\
\text { contig1569.344 }\end{array}$ & $\begin{array}{l}\text { evm.model. } \\
\text { contig1569.344 }\end{array}$ & $\begin{array}{c}\text { evm.model. } \\
\text { contig1569.344 }\end{array}$ & $\begin{array}{l}\text { evm.model. } \\
\text { contig1569.344 }\end{array}$ \\
\hline & $\begin{array}{c}\text { tigoo000058:1027588- } \\
1028906\end{array}$ & $\begin{array}{c}\text { tigoo000058:1027588- } \\
1028906\end{array}$ & $\begin{array}{c}\text { tigoo000058:1027588 } \\
1028906\end{array}$ & \\
\hline & $\begin{array}{c}\text { tigoooooos8:1116044- } \\
1116494\end{array}$ & $\begin{array}{c}\text { tigoooooo58:1116044- } \\
1116494\end{array}$ & $\begin{array}{c}\text { tigooooo058:1116044- } \\
1116494\end{array}$ & \\
\hline & $\begin{array}{l}\text { tigo0000151:403362- } \\
404089\end{array}$ & $\begin{array}{c}\text { tig00000151:403362- } \\
404089\end{array}$ & $\begin{array}{c}\text { tig00000151:403362- } \\
404089\end{array}$ & \\
\hline & tigooo17428:835657- & tigooo17428:835657- & tigooo17428:835657- & \\
\hline & 837290 & 837290 & 837290 & \\
\hline & $\begin{array}{l}\text { tig0o000058:1220542- } \\
1226128\end{array}$ & & & \\
\hline & $\begin{array}{l}\text { tigooooo198:12352- } \\
\qquad 12816\end{array}$ & & & \\
\hline
\end{tabular}

\section{In planta expression analysis of the candidate avirulence effector genes}

We anticipate that the avirulence gene that encodes the effector that activates $V 2$ resistance in tomato should be expressed in planta, at least in tomato. Based on deep transcriptome sequencing of Nicotiana benthamiana plants inoculated with V. dahliae strain JR2 it was previously demonstrated that the two effector candidates are expressed in planta (de Jonge et al. 2013). Real-time PCR analysis on a time course of tomato plants inoculated with the $V$. dahliae JR2 strain revealed that the two candidate genes are also expressed during tomato 
colonization, with a peak in expression around 7 days post inoculation, whereas little to no expression could be recorded upon growth in vitro (Fig. 2). Both genes are similarly expressed in $V$. dahliae strain $\mathrm{TO} 22$, albeit that the expression peaks slightly later, at $11 \mathrm{dpi}$ (Fig. 2). However, whereas the expression level of both genes is similar in $V$. dahliae strain JR2, Evm_344 is higher expressed in V. dahliae strain TO22 than XLOC_00170. Importantly, none of the six additional effector gene candidates that we identified in our comparative genomics approaches I-III and that are absent from $V$. dahliae strain JR2 is expressed in planta in V. dahliae strain TO22 (Fig. 2). Thus, these six effector genes can be disqualified as Avr candidates based on the transcriptional profiling, and only two genes remain, XLOC_00170 and Evm_344, that display an expression profile that is expected for a potential avirulence effector gene (de Jonge et al. 2011).

\section{Occurrence of the two candidate avirulence effector genes in a collection of} V. dahliae strains

To determine which of the two remaining effector genes encodes the genuine Avr molecule, a fungal isolate that carries either of the candidates separately should be tested for the ability to cause disease on Aibou plants that carry V2. Therefore, we attempted to identify a $V$. dahliae isolate that carries one of the two effector candidates only. To this end, presence/ absence polymorphisms were assessed in a collection of 52 previously sequenced $V$. dahliae strains (Fig. 3; de Jonge et al., 2012; Faino et al., 2015; Fan et al., 2018; Gibriel et al., 2019; Kombrink et al. 2017). Remarkably, both effector genes always occurred together in 17 of the isolates, including the four race 2 isolates that were sequenced in this study, and we were not able to identify any isolate that carries only a single of the two effector genes (Fig. 3). Thus, we were not able to disqualify either of the two effector genes as Avr candidate based on pathogenicity testing of naturally occurring $V$. dahliae isolates on Aibou plants. As many Avr effectors are found to be under selection pressure, and thus often display enhanced allelic variation (Stergiopoulos et al., 2007), we assessed the allelic variation of the two candidates in the 17 isolates in which they are present (Fig. 3). However, whereas no allelic variants were found for Evm_344, only a single allelic variant was found for XLOC_00170, namely a single nucleotide polymorphism in exon 3 leading to an amino acid substitution $\left(E_{73} V\right)$ in the effector protein that occurred in 7 of the 17 isolates that carry the effector gene (Fig. 4). Thus, also based on allelic variation none of the two effector genes can be disqualified as Avr candidate. 
(a)

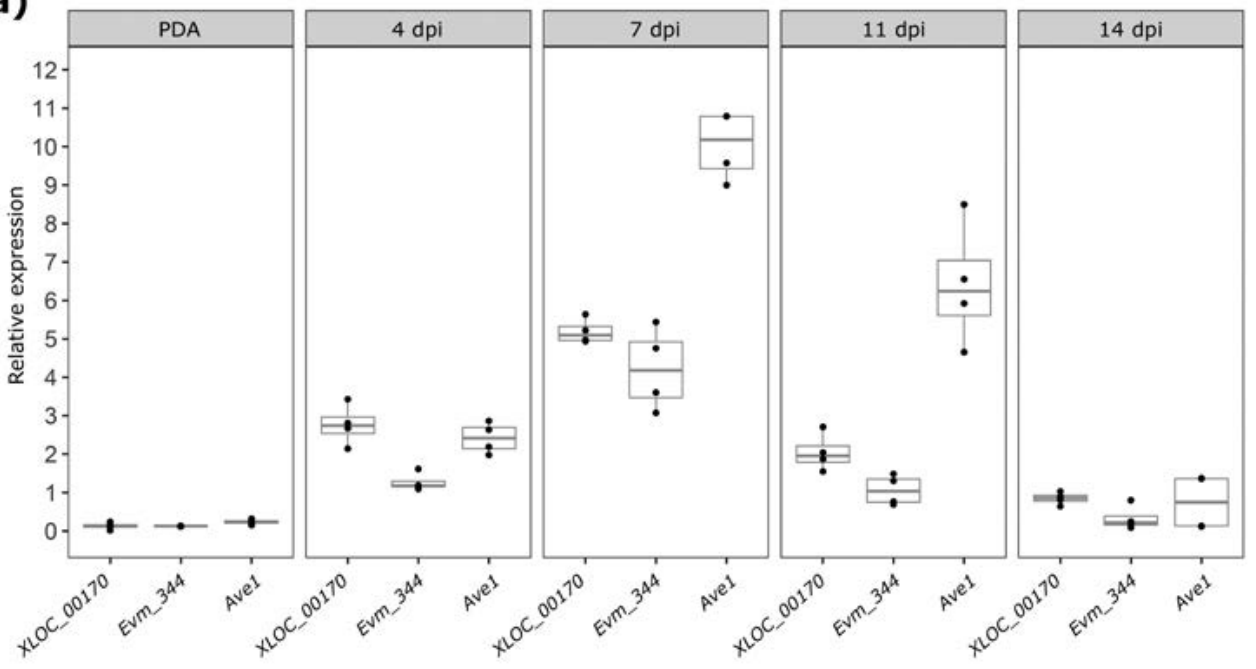

(b)

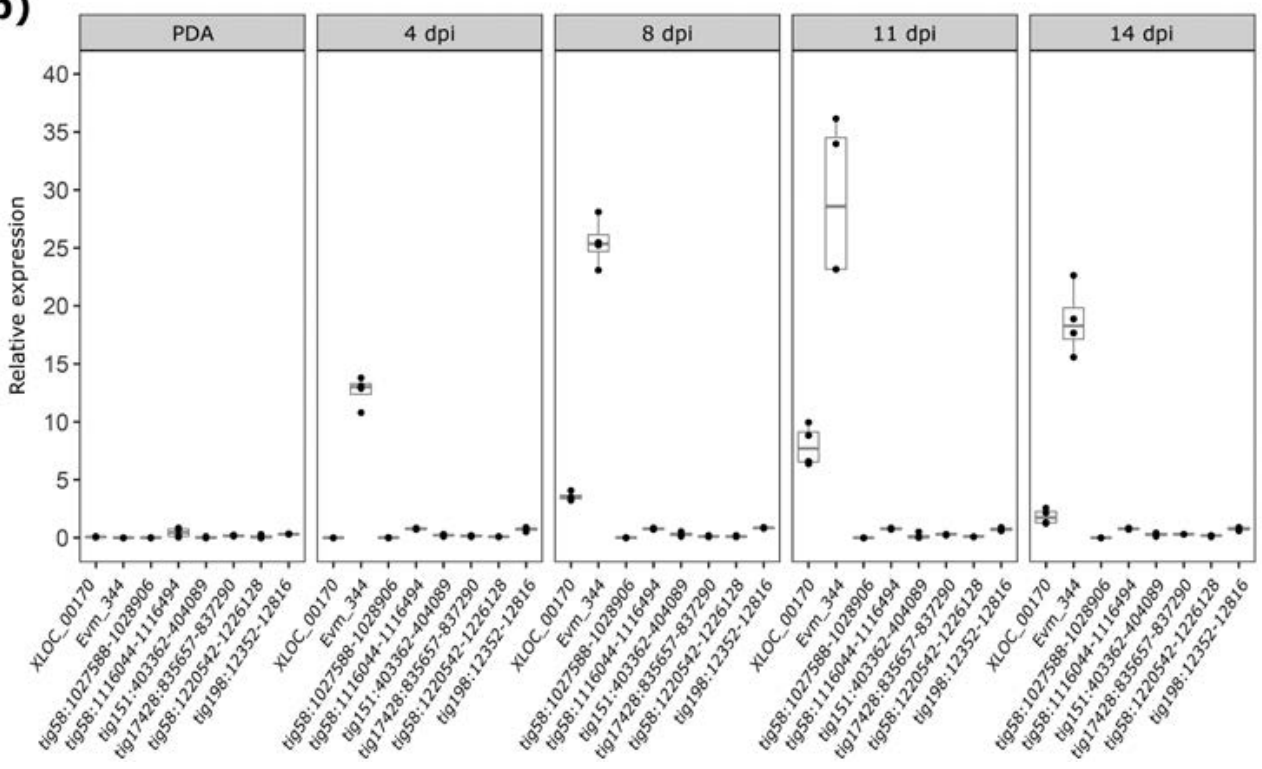

FIGURE 2 | Expression of V. dahliae candidate avirulence effector genes in vitro and during colonization of tomato plants. To assess in planta expression, twelve-day-old tomato Moneymaker seedlings were root-inoculated with $V$. dahliae strain JR2 (A) or strain TO22 (B), and plants were harvested from 4 to 14 days post inoculation (dpi), while conidiospores were harvested from five-day-old cultures of $V$. dahliae on potato dextrose agar (PDA) to monitor in vitro expression. Real-time PCR was performed to determine the relative expression of XLOC_O0170, Evm_344 and the race 1-specific effector gene Ave1 as a positive control (de Jonge et al., 2012) for strain JR2, using V. dahliae GAPDH as reference (A). Similarly, the relative expression of XLOC_00170, Evm_344 and six additional avirulence effector genes for strain TO22, using V. dahliae GAPDH as reference (B). 
The co-occurrence of both Avr effector candidate genes suggests that they are physically linked in the genome. Therefore, the genomic organization surrounding these two effector gene candidates was investigated based on the gapless genome assembly of $V$. dahliae strain JR2 (Faino et al., 2015). As expected, both genes are located in close proximity, namely in a previously identified LS region on chromosome 4 with only two other genes in between (Fig. 5). Furthermore, as typically observed in LS regions that are enriched in repetitive elements (de Jonge et al., 2013; Faino et al., 2016), the genes are surrounded by repeats (Fig. 5). This organization suggests that it may be unlikely to find naturally occurring $V$. dahliae isolates that carry only one of the two effector genes, so other strategies than testing natural isolates on Aibou plants for confirming which of the two candidates encodes the genuine Avr should be pursued.

\section{Phylogeny of 52 sequenced strains of $V$. dahliae revealed a close relationship among the strains carrying the Avr candidate genes}

To assess the phylogenetic relationships between strains that carry the two Avr candidates identified in this study and strains lacking these candidates a phylogenetic tree was generated using Realphy (Langmead and Salzberg 2012) with all V. dahliae genomes that are available in our laboratory. The phylogenetic tree shows that the strains group into three major clades, two of which contain strains with the two Avr candidate genes. However, within these clades closely related strains occur that lost the effector genes, demonstrating the occurrence of multiple independent losses (Fig. 3). Interestingly, strains carrying the allelic variant of XLOC_00170 are clustered in the same branch, suggesting that a single event caused this allelic variant (Fig. 3). Overall, we could not identify a clear phylogenetic structure within the $V$. dahliae population with respect to occurence of the effector gene. 


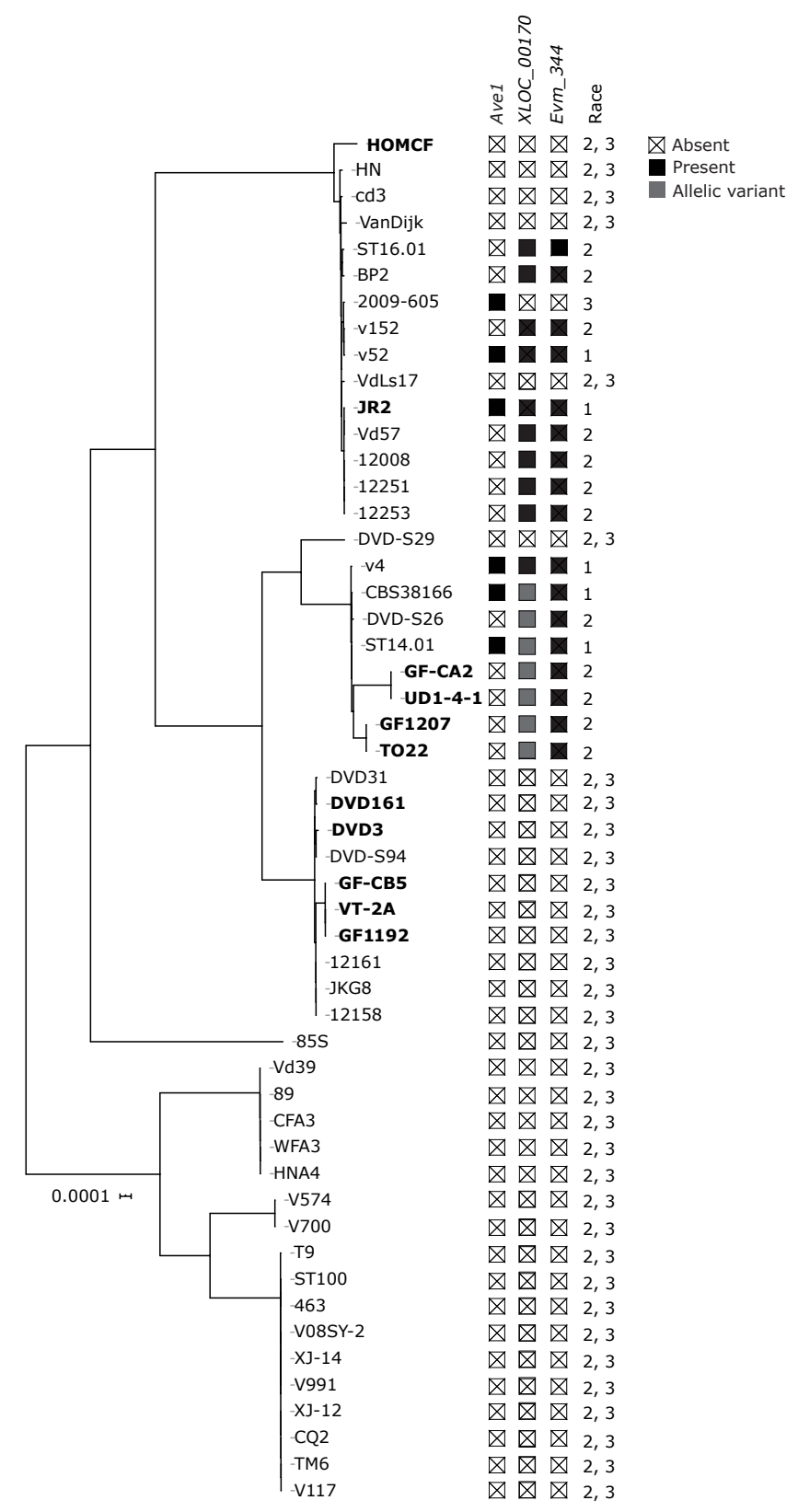

FIGURE 3 | Phylogenetic tree of sequenced V. dahliae strains with indication of presence-absence variation in race 1 and race 2 effector candidates. Strains that were phenotyped and included in any of the comparative genomics approaches (Table 2) are shown in bold. Presence of the avirulence gene Ave1, avirulence candidate genes XLOC_00170 and Evm_344, and the race designation based on presence or absence of these genes are indicated. Phylogenetic relationships between sequenced $V$. dahliae strains were inferred using Realphy (Langmead and Salzberg 2012). 
(a)

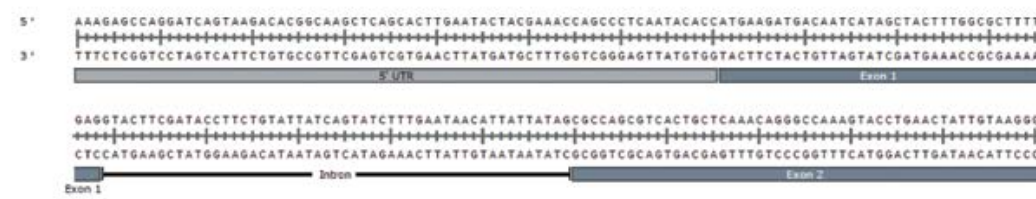

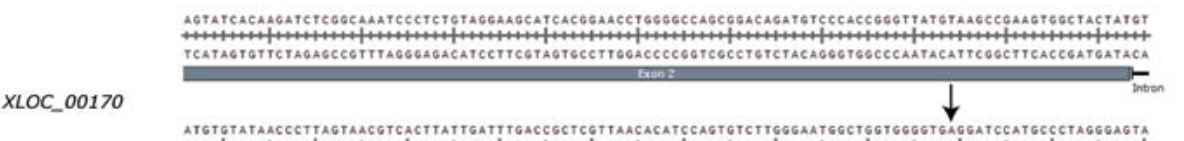

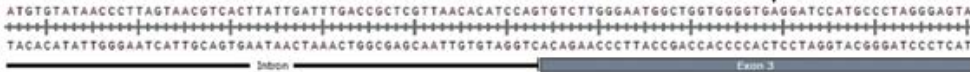
TGOAAATGGCTITGAGOTTOTTAGTATGTGAATCTAAGCCCOAGTCAGCACCTCTCAGOCACTGAGATTOTTACTAATCOTTGTAGTCTYTAACGTATAAAGOTA ACCTTTACCGAACTCCAACAATCATACACTTAGATTCGOGTCAGTCGTGGAGAGTCCGTOACTCTAACAATGATTAGCAACATCAQAAATTGCATATTTCCAT

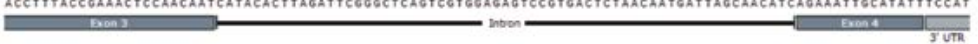

TACTTCTATTCAACTC S:

ATGAAGATAAGTTOAS S.

(b)
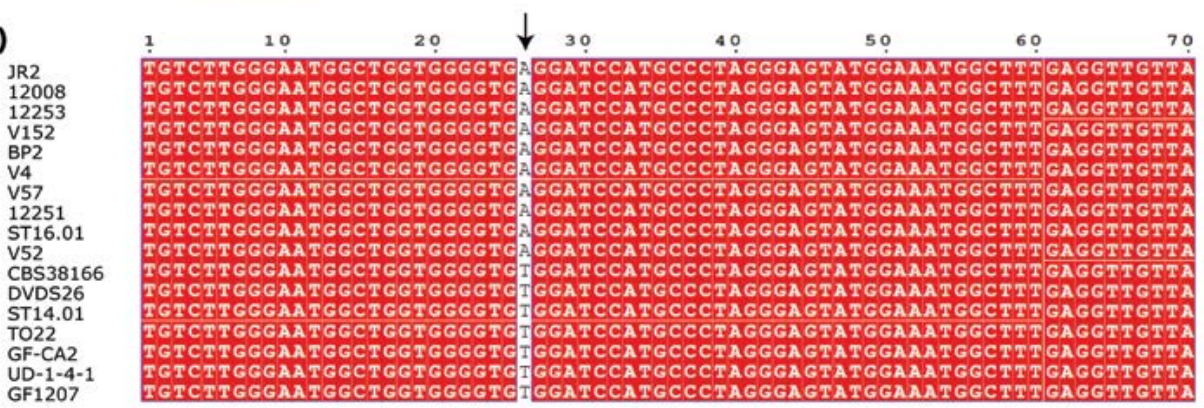

(c) JR2

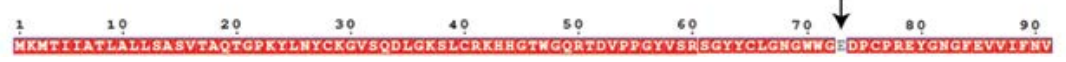

FIGURE 4 | Allelic variation of the effector candidate gene XLOC_00170 in sequenced strains carrying the effector. (A) Genomic sequence of effector candidate gene XLOC_00170, the arrow shows the position of the single nucleotide substitution found in particular strains. (B) Alignment of exon 3 of the effector candidate gene in the 17 strains containing the effector. The arrow shows the single nucleotide substitution that occurs in seven of the strains when compared with strain JR2. (C) Amino acid sequence of the effector candidate gene XLOC_00170 as encoded by V. dahliae strain JR2 with $\mathrm{E}_{73}$ that is substituted by $\mathrm{V}$ in seven isolates indicated by an arrow. 
(a)

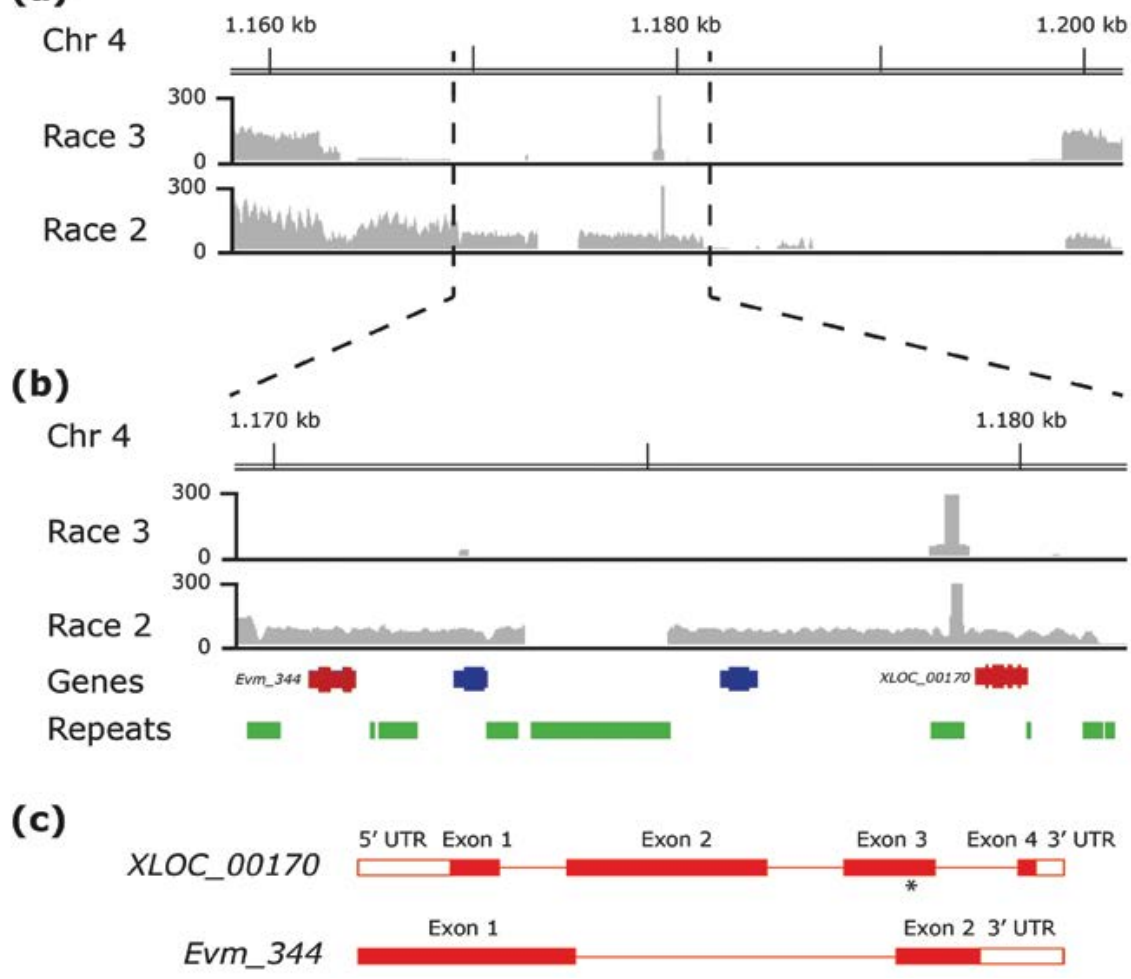

FIGURE 5 | Genomic organization of the locus comprising the two candidate Avr effector genes in V. dahliae strain JR2. (A) Schematic representation of the genomic region on chromosome 4 that is largely lacking in the race 3 strains that are included in comparative genomics approach IV (Table 2). Reads mapping to the region are shown as coverage plots for race 3 and race 2 strains. (B) Close-up of the region surrounding the Avr effector gene candidates XLOC_00170 and Evm_344 that are indicated as red gene models. Other gene models are show in blue and repetitive elements in green. (B) Gene models for XLOC_00170 and Evm_344. The asterisk indicates the approximate position of the single (A to T) nucleotide substitution in the XLOC_00170 gene of 7 of the 17 isolates that carry the gene, leading to a single amino acid substitution $\left(\mathrm{E}_{73} \mathrm{~V}\right)$.

\section{Discussion}

Currently, two major resistance gene sources have been described in tomato against the vascular wilt pathogen V. dahliae; Ve1 and the recently described V2 locus (Fradin et al. 2009; Usami et al. 2017). Since its initial introduction from a wild Peruvian tomato accession into agricultural crops in the 1950s (Deseret News and Telegram 1955), Ve1 has been widely used to control race 1 strains of $V$. dahliae. After multiple traditional approaches were unsuccessful to identify the avirulence effector that is recognized by Ve1, a genome sequencing and comparative genomics analysis based on $V$. dahliae race 1 and 2 strains by de Jonge et al. 
(2012) revealed a single gene, VdAve1. A subsequent functional analysis confirmed that $V d A v e 1$ encodes the avirulence effector that is recognized by Ve1. In this study, we used a similar approach to attempt to identify the gene encoding the avirulence effector that is recognized by $\mathrm{V} 2$. Theoretically, the failure of $\mathrm{V} 2$ to recognize "Av2" in race 3 strains could be due to several factors, such as single nucleotide polymorphisms, frameshift mutations or absence of expression of $A v 2$ in race 3 strains. However, effector evolution in $V$. dahliae seems to be often driven by presence/absence variations. For example, the VdAver effector which is recognized by $V e 1$ is completely absent in race 2 strains, while all race 1 strains thus far showed an identical copy of $V d A v e 1$ (de Jonge et al. 2012). Similarly, the recently identified pathogenicity effector "TomI", which is required for $V$. dahliae strains to infect tomato, was absent in all tested strains which were not pathogenic on tomato (Li 2019). Therefore, we hypothesized that the pathogenicity of race 2 and 3 strains on V2-resistant plants is governed by a similar presence/absence polymorphism of Av2. Through pathogenicity profiling on Aibou plants that carry the Ve1 and the V2 locus, we categorized multiple V. dahliae strains into race 2 and 3, namely those that are contained and those that cause disease, respectively. Sequencing and comparative genomics analysis followed by in planta expression analysis revealed $277 \mathrm{~kb}$ of genomic sequence that is specific to race 2 strains, and that lacks in race 3 strains. This sequence contains six genes that encode secreted proteins, of which only two were expressed in planta; XLOC_00170 and Evm_344. Likely, one of these two genes encodes the avirulence effector that is recognized by $V 2$.

To confirm which of these two effector genes encodes the genuine Avr molecule, $V$. dahliae isolates which carry either one of the effector candidates individually should be tested for their ability to cause disease on V2 plants. However, as the genes are located in close proximity, naturally occurring $V$. dahliae strains which carry only one of the effector genes may be difficult to find. Therefore, to test the role of either effector candidate in activating disease resistance in V2-resistant plants, genetic deletion- and complementation experiments should be performed, similar to the functional analysis performed by de Jonge et al. (2012) to confirm the nature of $V d A v e 1$ as avirulence factor. By generating individual deletion lines for each candidate in a $V$. dahliae race 2 strain, combined with individual introduction in a race 3 strain, it can be tested which of the two genes encodes the avirulence molecule; deletion of the genuine avirulence molecule from a race 2 strain, and introduction into a race 3 strain will result in gain- and loss of virulence on V2 tomatoes, respectively. To provide further evidence for a role as avirulence molecule, heterologous expression in V2 plants, for instance through potato virus $\mathrm{X}$ expression (Takken et al. 2000), could be performed to determine which of the two effector candidates is recognized and triggers a hypersensitive response on V2.

Like VdAve1, both XLOC_oo170 and Evm_344 reside in a lineage-specific region of the V. dahliae genome. In filamentous plant pathogens, effector genes tend to be enriched in such lineage-specific regions (Dong et al. 2015). Typically, these lineage-specific regions are also 
enriched in repetitive and transposable elements, causing these regions to be highly flexible, facilitating effector evolution (De Jonge et al. 2013; Faino et al. 2016; Dong et al. 2015). One important evolutionary advantage of this genomic flexibility is the ability of pathogens to lose effector molecules that are located in these regions once they become recognized and therefore have become avirulence molecules. For example, for VdAve1, the most likely evolutionary scenario is that this effector was acquired once from plants in $V$. dahliae through horizontal gene transfer, and subsequently lost multiple times in independent lineages that encountered VeI-resistant plants (Faino et al. 2016; de Jonge et al. 2012). Probably, the genomic flexibility of the LS region in which VdAver resides has contributed to facilitate these presence/absence polymorphisms of $V d A v e 1$. Thus far, all studied race 1 strains carry an identical copy of $V d A v e 1$, while all race 2 strains lack the complete VdAver gene (de Jonge et al. 2012; Faino et al. 2016; De Jonge et al. 2013). For XLOC 00170, we observed two allelic variants due to the occurrence of a SNP that results in a single amino acid substitution. However, if XLOC 00170 encodes the avirulence molecule, both variants will be recognized because strains with either of the allelic variants were categorized as race 2 in our pathogenicity assays. All $V$. dahliae race 2 strains included in our study carry XLOC_00170 and Evm_344, while all race 3 strains lack both entire genes, suggesting that evasion of race 2 resistance is based on presence-absence variation similar to the presenceabsence variation of $V d A v e 1$ between race 1 and race 2 strains, respectively.

Further analyses of the $A v 2$ molecule may reveal relevant information with respect to effective management of $V$. dahliae race 2 and race 3 strains. After the introduction of Ve1-resistant cultivars in the mid-1950s, race 2 strains emerged rapidly, first in the US (Alexander 1962; Robinson 1957), and soon thereafter also in Europe (Cirulli 1969; Pegg and Dixon 1969). After V2 resistance was introduced in Japan in 2006 (Usami et al. 2017), V2-resistance overcoming race 3 strains emerged in several Japanese prefectures on two separate islands. Assuming that either XLOC_00170 or Evm_344 encodes the avirulence molecule that is recognized by V2, our analyses provide additional clues about the distribution of race 3 strains outside Japan, and thus can help to predict to what extent race 2 resistance can be successfully employed to manage Verticillium wilt disease. The strains that lack XLOC_00170 and Evm_344 are found worldwide, as they comprise strains that were originally isolated in China, Canada, the USA, and Europe, including the Netherlands. This indicates that, besides in Japan, race 3 strains are already present worldwide and that $V e 1$ and $V 2$ may not be sufficient to control $V$. dahliae in these regions. However, more strains should be tested to obtain a more complete picture of the worldwide distribution of race 2 and 3 strains. Finally, our observations suggest that the presence-absence variation for these two genes is not caused by selection pressure imposed by the V2 gene, as this gene has likely not yet been exploited outside Japan. Possibly, the presence-absence variation of these genes is caused by other factors.

Further functional analysis of the avirulence molecule recognized by V2 may also provide 
initial clues about the potential durability of $V 2$ resistance. For the durability of the resistance governed by an immune receptor, an important factor is a significant fitness penalty for the pathogen upon losing the corresponding avirulence factor (Brown 2015). Often, effectors of plant pathogens display functional redundancy, causing mutation or deletion of effectors to not always result in a significant fitness cost (Win et al. 2012; de Jonge et al. 2011). If such functionally redundant effectors become recognized by an immune receptor, they can be lost with relatively little cost to the pathogen. However, deletion of other effectors, such as VdAve1, can cause a significant virulence penalty (de Jonge et al. 2012), making resistance triggered by these avirulence effectors in theory more durable. Therefore, it is relevant to test whether deletion of XLOC_00170 or Evm_344 imposes a significant virulence penalty as observed for VdAve1 (de Jonge et al. 2012). However, the absence of a clear difference in aggressiveness between race 2 and race 3 strains as observed in our phenotyping, which is in contrast to the significant difference in virulence as previously observed between race 1 and race 2 strains (de Jonge et al., 2012) suggests that the contribution of XLOC_00170 and Evm_344 to V. dahliae virulence in race 2 strains is modest.

Finally, the avirulence effector recognized by V2 may be used as a tool for genetic mapping of the V2 gene. Typically, $V$. dahliae symptoms on tomato display a high degree of variability, making genetic mapping analyses for $V$. dahliae resistance challenging. If the recognition of the avirulence effector triggers a robust hypersensitive response on V2 plants, this phenomenon may be used for phenotyping in genetic mapping analyses. A similar effectorassisted resistance breeding approach has been used successfully in the identification of $R$ genes in wild tomato accessions against the late blight pathogen Phytophthora infestans (Vleeshouwers and Oliver 2014; Du et al. 2015), as well as against the leaf mould pathogen Cladosporium fulvum (Takken et al. 1999; Lauge et al. 1998). It may thus be worth investigating whether such an effector-assisted approach may also help in the identification of the V2 gene.

\section{Materials \& methods}

\section{V. dahliae inoculation and phenotyping}

Plants were grown in potting soil (Potgrond 4, Horticoop, Katwijk, the Netherlands) under controlled greenhouse conditions (Unifarm, Wageningen, the Netherlands) with day/night temperature of $24 / 18^{\circ} \mathrm{C}$ for $16-\mathrm{h} / 8$-h periods, respectively, and relativity humidity between 50 and $85 \%$. For V. dahliae inoculation, 10-day-old seedlings were root-dipped for $10 \mathrm{~min}$ as previously described (Fradin et al. 2009). Disease symptoms were scored at 21 days post inoculation (dpi) by measuring the canopy area to calculate stunting as follows:

$$
\text { stunting }(\%)=\left(1-\frac{\text { canopy area } V . \text { dahliae }- \text { inoculated plant }}{\text { average canopy area of mock }- \text { inoculated plants }}\right) * 100
$$


To test for significant differences in stunting, an ANOVA was performed to test for significant stunting. Outliers were detected based on the studentized residuals from the ANOVA analysis. Datapoints with studentized residuals below -2.5 or above 2.5 were considered as outliers and removed.

\section{High Molecular Weight (HMW) DNA isolation and Nanopore sequencing}

Conidiospores were harvested from potato dextrose agar (PDA) plates, transferred to Czapek dox medium and grown for ten days. Subsequently, fungal material comprising mycelium and conidiospores was collected on Miracloth, freeze-dried overnight and ground to powder with mortar and pestle of which $300 \mathrm{mg}$ was incubated for one hour at $65^{\circ} \mathrm{C}$ with $350 \mu \mathrm{L}$ DNA extraction buffer ( $0.35 \mathrm{M}$ Sorbitol, $0.1 \mathrm{M}$ Tris-base, $5 \mathrm{mM}$ EDTA pH 7.5), $350 \mu \mathrm{L}$ nucleic lysis buffer (0.2 M Tris, $0.05 \mathrm{M}$ EDTA, $2 \mathrm{M} \mathrm{NaCl}, 2 \% \mathrm{CTAB}$ ) and 162.5 $\mu \mathrm{L}$ Sarkosyl (10\% w/v) with $1 \% \beta$-mercaptoethanol. Next, $400 \mu \mathrm{L}$ of phenol/chloroform/ isoamyl alcohol (25:24:1) was added, shaken manually and incubated at room temperature (RT) for 5 minutes before centrifugation at $16,000 \mathrm{~g}$ for $15 \mathrm{~min}$ at RT. After transfer of the aqueous phase to a new tube, $10 \mu \mathrm{l}$ of RNAase $(10 \mathrm{mg} / \mu \mathrm{L})$ was added and incubated at $37^{\circ} \mathrm{C}$ for one hour. Subsequently, half a volume of chloroform was added, shaken manually and centrifuged at 16,000 g for $5 \mathrm{~min}$ at RT. After transfer of the aqueous phase to a new tube the chloroform extraction was repeated. Next, the aqueous phase was mixed with 10 volumes of $100 \%$ ice-cold ethanol, incubated for $30 \mathrm{~min}$ at RT, and the DNA was fished out using a glass hook and transferred to a new tube containing $500 \mu \mathrm{L}$ of $70 \%$ ethanol, wash step with $70 \%$ ethanol was performed twice. Finally, the DNA was air-dried, resuspended in nuclease-free water and stored at $4^{\circ} \mathrm{C}$ for two days. The DNA quality, size and quantity were assessed by nanodrop, gel electrophoresis and Qubit analyses, respectively.

Library preparation with the Rapid Sequencing Kit (SQK-RAD004) was carried out as described by the manufacturer (Oxford Nanopore Technologies, Oxford, UK) with an initial amount of 400 ng HMW DNA. An R9.4.1 flow cell (Oxford Nanopore Technologies, Oxford, UK) was loaded with the library and run for 24 hours. Base calling was performed using Guppy (version 3.1.5; Oxford Nanopore Technologies, Oxford, UK) with the high accuracy base calling algorithm. Next, adapter sequences were removed using Porechop (version 0.2.4 with default settings; Wick, 2018). Finally, the reads were self-corrected, trimmed and assembled using Canu (Version 1.8; Koren et al., 2017).

\section{Comparative genomics and candidate identification}

Self-corrected reads from the $V$. dahliae race 3 strains were mapped against the designated reference genome using BWA-MEM (version 0.7.17; default settings; Li, 2013). Reads with low-quality mapping score (score less than 10) were removed using Samtools view (version 1.9; setting: - q 10) (Li et al. 2009), and also reads mapping in regions with low coverage ( $<10 \mathrm{x})$ were discarded using Bedtools coverage (version 2.25.0; setting: - $d$ ) (Quinlan and Hall 2010). 
Next, self-corrected reads from race 2 strains were mapped against the retained reference genome-specific regions that are absent from the race 3 strains. Retained sequences that were shared by the reference and every race 2 strain while being absent from every race 3 strain were retained as candidate regions to encode the Avr molecule.

The previously determined gene annotation of the $V$. dahliae strain JR2 genome (Faino et al. 2015) was used to extract genes when $V$. dahliae strains JR2 or TO22 were used as alignment references. To this end, the retained sequences shared by the $V$. dahliae strain TO22 reference assembly as well as the race 2 strains that are absent from the race 3 strains were mapped against the $V$. dahliae strain JR2 genome assembly, and genes present in the shared sequences were extracted. The remaining sequences that did not map to the $V$. dahliae strain JR2 genome assembly were annotated using Augustus (version 2.1.5; default settings; Stanke et al., 2006). Next, SignalP software (version 4.0; Petersen et al. 2011) was used to identify $\mathrm{N}$-terminal signal peptides in the predicted protein sequences.

\section{Real-time PCR to detect expression of candidate genes}

To determine expression profiles of Avr effector candidate genes during $V$. dahliae infection of tomato, two-week-old tomato (Moneymaker) seedlings were inoculated with $V$. dahliae strain JR2 or with strain TO22, and stems were harvested at regular intervals up to 14 days post inoculation (dpi). To assess expression in vitro, conidiospores were harvested from five-day-old PDA plates. Total RNA extraction and cDNA synthesis were performed as previously described (Santhanam et al. 2013). Real time-PCR was performed with primers listed in Table S1, using the $V$. dahliae glyceraldehyde-3-phosphate dehydrogenase gene $(\mathrm{GAPDH})$ as endogenous control.

\section{Genome mining}

In total, 44 previously sequenced $V$. dahliae strains and the eight strains sequenced in this study were mined for the presence of Avr effector gene candidates using BLASTn. Next, gene sequences were extracted using Bedtools (setting: getfasta) (Quinlan \& Hall, 2010) and aligned to determine allelic variation using Espript (version 3.0; default settings) (Robert and Gouet 2014). Similarly, amino acid sequences were aligned using Espript (Robert and Gouet 2014).

To determine the genomic localization of XLOC_00170 and Evm_344, the genome assembly and annotation of $V$. dahliae strain JR2 was used (Faino et al. 2015) together with coverage plots from reads of race 3 and race 2 strains as described in comparative genomics approach IV (Table 2) using R scripts, with package karyoploteR for R (version 3.6) using kpPlotBAMCoverage function. A schematic representation of the genomic region on chromosome 4 comprising XLOC_00170 and Evm_344 was generated using Integrative Genomics Viewer (IGV) software v2.6.3 (Robinson et al. 2011). 


\section{Phylogenetic tree construction}

A phylogenetic tree of 52 sequenced $V$. dahliae strains was generated with Realphy (version 1.12) (Bertels et al., 2014) using Bowtiez (version 2.2.6) (Langmead and Salzberg 2012) to map genomic reads against the reference $V$. dahliae strain JR2. A maximum likelihood phylogenetic tree was inferred using RAxML (version 8.2.8) (Stamatakis 2014).

\section{Functional Analysis}

For heterologous expression in tomato, the binary pSfinx vector for potato virus X-mediated expression was used (Takken et al. 2000). To this end, the XLOC_00170 and Evm_344 coding sequences were amplified from genomic DNA of $V$. dahliae strain TO22 and cloned into pSfinx (Takken et al. 2000). Agrobacterium tumefaciens transformation and inoculations on Moneymaker plants that lack known $V$. dahliae resistance genes, on VeI-transgenic Moneymaker plants (Fradin et al. 2009), and on Aibou plants that carry Ve1 and V2 (Usami et al. 2017) were performed as described previously (van Esse et. al., 2006). 


\section{Supplemental material}

TABLE S1 | Primers used in this study.

\begin{tabular}{ll}
\hline Primer name & Oligonucleotide sequence $\left(5^{\prime} \rightarrow 3^{\prime}\right)$ \\
\hline XLOCoo170-F & CAGCCCTCAATACACCATGAAGATG \\
\hline XLOCool70-R & TTCCGTGATGCTTCCTACAGAGG \\
\hline evm1569.344-F & CACTTGCTTGGTTGCATGAT \\
\hline evm1569.344-R & TCCTTACTGTGCTGGATTCG \\
\hline VdAve1-F & AGCTTTCTACGCTTGGA \\
\hline VdAve1-R & TTGGCTGGGATTGCT \\
\hdashline VdGAPDH-F & CGAGTCCACTGGTGTCTTCA \\
\hline VdGAPDH-R & CCCTCAACGATGGTGAACTT \\
\hline
\end{tabular}





\title{
Chapter 7
}

\section{General discussion}

\author{
Jasper P. Vermeulen ${ }^{1,2}$
}

'Laboratory of Phytopathology, Wageningen University \& Research, 6708 PB Wageningen, The Netherlands.

${ }^{2}$ Laboratory of Plant Breeding, Wageningen University \& Research, 6708 PB Wageningen, The Netherlands. 


\section{Introduction}

Verticillium dahliae is a destructive fungal vascular pathogen that can cause severe yield losses on many different crop species (Tabaeizadeh et al. 1999; Zeise and Von Tiedemann 2002; Klosterman et al. 2009; Fradin and Thomma 2006). One of the preferred strategies to control this disease has been the identification and employment of genetic resistance. Wild crop relatives have provided breeders with a rich pool of genetic resources to improve crops with many traits, including disease resistances (Hajjar and Hodgkin 2007; Rick and Chetelat 1995). Thus far, over 40 resistance genes against different pathogens and pests have been introgressed from wild tomato species, including S. peruvianum, S. cheesmanii, S. pimpinellifolium and various other wild species into tomato cultivars (Rick and Chetelat 1995). It has been estimated that every year at least one additional $R$ gene is introduced into cultivated tomato from a wild tomato species (Rick and Chetelat 1995). Like for resistance against many other tomato pathogens, wild tomato relatives have also been used as a source of resistance against Verticillium dahliae. Indeed, the first identified $R$ gene against Verticillium, Ve1, was derived from a wild Peruvian tomato (Schaible et al. 1951). In an attempt to identify additional resistances against $V$. dahliae, Yadeta (2012) performed a large-scale wild germplasm screening, which revealed several wild tomato accessions that provided resistance against race 2 strains of $V$. dahliae. In $m y \mathrm{PhD}$ research, we further evaluated the resistance of six of these wild tomato accessions and performed QTL analyses to determine whether the resistance of these accessions could be used in breeding programs to generate tomato cultivars with a more broad resistance against $V$. dahliae.

\section{Symptoms of Verticillium vascular wilt disease: wilting and stunting}

In resistance screens, and especially in QTL analyses, the methodology to score disease symptoms is of crucial importance. Resistances and QTLs for disease resistance can only be accurately identified based on accurately scored disease symptoms or pathogen colonization. Therefore, it is very important to determine beforehand which symptoms will be scored, and with which methods. Since V. dahliae resides inside the plant for most of the disease cycle, typically only symptoms of the infection are scored in QTL analyses. Still, techniques exist to quantify the colonization of $V$. dahliae through real-time PCR, or to assess fungal presence with fungal outgrowth assays from diseased tissues (Lievens et al. 2006; Fradin et al. 2009). However, as these techniques require tissue samples to be processed, they are more laborious than direct measurements of $V$. dahliae symptoms. Therefore, quantifying the colonization of $V$. dahliae is too laborious for the analysis of entire mapping populations for QTL analyses. 
Disease symptoms caused by Verticillium dahliae largely depend on the host species, environment, and the age of the host plant (Fradin and Thomma 2006). There are no unique symptoms that occur consistently on all plant species infected by $V$. dahliae. Commonly reported symptoms for Verticillium wilt diseases are foliar symptoms, such as chlorosis, necrosis and senescence of the leaves (Fradin and Thomma 2006), vascular browning, and stunting; growth retardation of $V$. dahliae infected plants. In resistance screens and QTL mapping studies for $V$. dahliae resistance, various different types of symptoms have been used. In QTL analyses for resistance against Verticillium spp. in cotton, oilseed rape, strawberry and alfalfa, foliar and/or vascular symptoms such as yellowing, chlorosis and necrosis were most commonly used, typically using a numeric scale, i.e. from o to 5 (Bolek et al. 2005; Wang et al. 2008; Fang et al. 2013, 2014; Jiang et al. 2009; Zhang et al. 2015, 2014b; Rygulla et al. 2008; Antanaviciute et al. 2015; Yu et al. 2020). In tomato, stunting symptoms have most commonly been used in resistance and pathogenicity studies (Yadeta 2012; Fradin et al. 2009; Kombrink et al. 2017; Zhang et al. 2014c; Fradin et al. 2014), although foliar symptoms have also been scored (Usami et al. 2017; Diwan et al. 1999), sometimes in combination with stunting (Schaible et al. 1951).

In resistance screens, the main aim is typically to compare the symptom expression of different host genotypes to draw conclusions about differences in resistance between the host genotypes. To best be able to draw such conclusions, the phenotyping approach should provide a high "discriminative power", so that differences in symptom expression between host genotypes can best be detected. In the conditions in our plant growing facilities, the susceptible control S. lycopersicum Moneymaker did not consistently display clear foliar symptoms such as yellowing, chlorosis or wilting. However, both in this $\mathrm{PhD}$ research and during previous studies (Yadeta 2012; Fradin et al. 2009; Kombrink et al. 2017; Zhang et al. 2014c; Fradin et al. 2014), Moneymaker plants consistently displayed stunting symptoms when inoculated with $V$. dahliae. Therefore, in resistance screens where the resistance of other tomato accessions is compared with that of Moneymaker, stunting symptoms generally provide a better discriminative power than foliar symptoms. Since stunting symptoms can be measured from different plant size-related parameters, in Chapter 2 we evaluated various of these parameters to determine which parameter gives the highest discriminative power. Of the size-related parameters in our analysis, canopy area measurements yielded the highest discriminative power. Compared to 14 days post inoculation, this discriminative power was further enhanced at 21 days post inoculation. Therefore, in this $\mathrm{PhD}$ research, disease screens were based on stunting symptoms which were calculated from canopy area measurements at 21 days after inoculation. 


\section{Validation of the V. dahliae resistance of three wild tomato accessions}

Of the 57 wild tomato genotypes that were screened by Yadeta (2012), eight displayed resistance against two race 2 strains of $V$. dahliae. In Chapter 3 , the resistance of several of these accessions was further validated with the phenotyping approach developed in Chapter 2 , which resulted in the selection of S. cheesmanii VG-20 and S. pimpinellifolium VG-21 as the most potent accessions for further analyses. Although $S$. pimpinellifolium VG-3 initially did not display a high level of resistance, the availability of a fully genotyped RIL population for this accession (Víquez-Zamora et al. 2014) motivated us to also select this accession for further analysis, while in parallel RILs were developed from VG-20 and VG-21. However, the development of novel RIL populations is a time-consuming procedure. Originally, Yadeta (2012) suggested that VG-20 and VG-21 may possess race-2-specific resistance, as both accessions displayed severe stunting symptoms and $V$. dahliae colonization in the stems when challenged with two aggressive race 1 strains. Although we confirmed in Chapter 3 that these accessions indeed displayed increased stunting when challenged with aggressive race 1 strains, we also demonstrated that VG-20, VG-21 and VG-3 were still less stunted than the susceptible S. lycopersicum Moneymaker control. This finding suggests that the resistance of these wild accessions is also effective against race 1 strains, albeit to a lesser extent. In fact, the resistance of the three wild accessions seems of a broad-spectrum nature, as the accessions displayed reduced symptom expression and $V$. dahliae colonization against the majority of the strains used in this $\mathrm{PhD}$ research. Given that the accessions also displayed reduced stunting symptoms when challenged with a $V$. nonalfalfa strain, this also indicates that the resistance is not solely specific to $V$. dahliae. This is not unique, as Ver was also found to be effective against multiple $V$. dahliae species (de Jonge et al. 2012).

Resistance against plant pathogens can be classified in qualitative and quantitative resistance. Qualitative resistance segregates into discrete classes of resistant and susceptible individuals and is typically based on a single $R$ or $S$ gene. In contrast, quantitative resistance segregates into a continuous distribution of phenotypes from susceptible to resistant and is, in most cases, based on multiple loci (Corwin and Kliebenstein 2017). When compared with qualitative $R$ gene resistance, quantitative resistance is commonly found to be more broadspectrum than $R$ gene-based resistance (Poland et al. 2009; Corwin and Kliebenstein 2017; Parlevliet 2002). This makes sense, since qualitative $R$ gene-based resistance is typically only effective against pathogens which possess the avirulence molecule that is recognized by the immune receptor that is encoded by the $R$ gene. Still, $R$ gene-based resistance can in some cases also be more broad-spectrum. For example, two NB-LRR immune receptors from wild potato species were found to govern relatively broad-spectrum resistance against potato late blight (Song et al. 2003; Van Der Vossen et al. 2003). Broad-spectrum R-gene resistance can occur when the Avr that is recognized by the R protein is widely conserved among pathogen strains, i.e. because the Avr is required for pathogenicity and therefore 
costly for pathogen strains to lose. However, based on the segregation of the stunting symptoms in the VG-20, VG-21 and VG-3 RIL populations, the resistance of these accessions is probably not based on a single $R$ gene. In a RIL population, resistance governed by a single $R$ gene should display a 1:1 segregation pattern, as RILs can either carry the $R$ gene and be resistant or lack the functional $R$ gene and be susceptible. Given that in all three populations the stunting symptoms displayed a continuous distribution between the most susceptible to the most resistant plants, it is unlikely that the resistance is based on a single $R$ gene. The resistance of these accessions is therefore quantitative and most likely based on the concerted action of multiple genes.

\section{The complexities of mapping quantitative resistance against V. dahliae}

When compared with qualitative $R$ gene resistance, quantitative resistance can be far more difficult to map (Corwin and Kliebenstein 2017). This is illustrated by the QTL analyses described this PhD research, as in two out of three RIL populations no QTLs could be identified for resistance based on stunting symptoms. In a QTL analysis, many factors influence the success and accuracy of the detection of QTLs, including population size, genetic properties of the QTLs, and accuracy of the phenotypic data (Collard et al. 2005). In a mapping population, the total variance of a phenotype is the result of the sum of underlying genetic variance and environmental variance (Acquaah 2012). When two individuals from a RIL population are selected randomly, the difference in a specific trait between these individuals is the result of genetic differences between the plants and differences in the environment the plants were exposed to. If a large proportion of the variance of the phenotype is not caused by genetic factors but by environmental factors, it is therefore generally more difficult to detect QTLs associated with the phenotype. The proportion of the phenotypic variance of a given trait that is caused by genetic factors is also referred to as the heritability of a trait (Acquaah 2012). In the three RIL populations screened in my $\mathrm{PhD}$ research, the estimated heritability of the stunting of $V$. dahliae-inoculated plants was rather low, ranging from $H^{2}=0.32$ for the VG-3, $H^{2}=0.17$ for the VG-20, and $H^{2}=0.10$ for the VG-21 RIL population (Chapter 4-5). Although these are only rough estimates of the actual heritability, these estimates suggest that a significant proportion of the variance in stunting was caused by environmental factors rather than by genetic factors. This is further underlined by the low reproducibility the phenotypic data between the two screens of each RIL population (Chapter 4-5). Probably, this low estimated heritability and reproducibility of the stunting symptoms combined with the probably polygenic nature of the resistance has made the detection of QTL in these populations cumbersome.

Although it is not exactly known what causes the stunting of $V$. dahliae-infected plants, stunting has been suggested to be caused by blockage of the xylem vessels, either by the 
fungus or the plants defence responses (Fradin and Thomma 2006). Possibly, water stress resulting from this vessel blockage causes stomatal closure, which subsequently reduces the photosynthetic activity. Since many environmental factors such as temperature, lightlevels, nutrition and humidity may affect growth, photosynthesis, water stress or stomatal conductance, local environmental variations in these factors could thus have caused variation in the stunting. Other factors such as toxins produced by the fungus may also cause stunting of V. dahliae-infected plants (Pegg and Brady 2002; Fradin and Thomma 2006; Buchner et al. 1989). It is likely that other environmental factors can cause significant variation in stunting. After all, many factors influence plant growth, and local variations in these factors can undoubtedly also cause variation in stunting. To reduce the effect of local environmental differences on the growth of the plants, the VG-20 and VG-21 RIL population was randomized in pairs of mock-inoculated and V. dahliae-inoculated RILs. This way, stunting of each $V$. dahliae-inoculated plant could be compared to an adjacently located mock-inoculated plant, which was therefore exposed to a similar micro-environment. However, as the estimated heritability of the stunting symptoms in the VG-20 and VG21 RIL population was still low despite this paired randomization approach, other factors must also have played a role as well. One factor which was especially apparent in the VG-21 RIL population was intumescence; a physiological disorder of the leaves characterized with tumour formation (Fig. 1) on the leaf surface which is caused by abnormal cell enlargement and cell divisions (Lang and Tibbitts 1983; Kubota et al. 2017). Several RILs in the VG-21 RIL population developed severe intumescence, resulting in necrosis and abscission of leaves, which affected stunting measurements in turn. Given that this intumescence was especially persistent in the VG-21 RIL population, this may explain why the estimated heritability of the resistance in this population was lower than in the other populations. In tomato plants grown in greenhouses, intumescence is often caused by of UV-B deficiencies (Kubota et al. 2017), since glass windows filter out most of the UV-B irradiance (Tuchinda et al. 2006). Fortunately, supplementation of UV-B light can reduce or even eliminate the intumescence (Kubota et al. 2017), and thereby probably increase the heritability of the stunting symptoms in the VG-21 RIL population.

Although as part of this $\mathrm{PhD}$ research we optimized the phenotyping approach and the randomizing design, other adaptations could possibly help to further increase the heritability or the precision of the QTL analysis. First of all, a straight-forward way to improve the accuracy of the phenotypic data would be to further increase the number of replicates per RIL, as this will result in better estimates of the phenotype of each RIL. Secondly, the identification and inclusion of covariates in the QTL analysis may enhance the QTL analysis. If a covariate has a significant effect on the phenotype, its inclusion in the QTL analysis will reduce the residual variation in the phenotype, and thereby enhance the ability to detect QTLs (Broman and Sen 2009). In Chapter 5 we found that there was a modest association between canopy area of mock-inoculated plants and the stunting symptoms, 
and that inclusion of the mock canopy area as a covariate resulted in the identification of an additional QTL. It may thus be helpful to search for additional covariates and test the effect of the inclusion of these covariates on the QTL analysis. For example, if local variations in humidity and temperature would indeed affect the stunting of plants, inclusion of the average humidity and temperature each RIL was exposed to as a covariate may further enhance the QTL analysis.

Another important factor which determines the success of a QTL analysis is the genetic architecture of a trait and the size of the mapping population. When a trait is highly polygenic and/or based on genes which individually only have small effects on the phenotype, larger mapping populations, ideally consisting of many hundreds or even thousands of genotypes, are desirable (Li et al. 2010; Vales et al. 2005). An increase in population size provides an increased statistical power, better estimates of gene effects, and more precise confidence intervals of the locations of the QTLs (Beavis 1998; Darvasi et al. 1993). When the population size is small, QTLs which are closely linked may appear as a single QTL or even remain completely undetected when the linked QTLs have opposing effects. Since the segregation of the resistance of VG-3, VG-20 and VG-21 suggests that these resistances are polygenic, increasing the number of RILs is a sensible step to improve the accuracy of the QTL analysis.

It needs to be acknowledged that an increase of both the number of RILs and the number of replicates per RIL will result in enormous experiments consisting of many thousands of plants. To make such large-scale experiments practically feasible, a high-throughput approaches needs to be adopted to be able to handle these large numbers of plants. Probably, the major bottleneck of such large-scale experiments would be the phenotyping, as all plants should ideally be phenotyped in a relatively short time period to allow reliable phenotypic comparison of the plants. Fortunately, the approach in this $\mathrm{PhD}$ research to measure the stunting of $V$. dahliae-infected plants based on image analysis can relatively easily be automated. In the last decade, an increasing number of automated plant phenotyping platforms were developed, many of which perform a high-throughput phenotyping analysis based on top-down images (Humplík et al. 2015). Such systems can therefore easily be adapted to allow high-throughput measurements of $V$. dahliae-induced stunting symptoms.

Fortunately, despite the difficulties during the QTL analyses for quantitative V. dahliae resistance, we were able to identify multiple QTLs in the Moneymaker x VG-20 RIL population (Chapter 5), of which three were derived from the VG-20 parent, and two originated from Moneymaker. It may seem surprising that also QTLs were identified from the susceptible parent. However, also Moneymaker plants are not completely derived of an immune system. In the initial phase of the infection, plant pathogens enter the apoplast, the extracellular space of plant tissue. As a first layer of basal defence, plants employ toxins and hydrolytic enzymes in the apoplast, such as chitinases and glucanases to inhibit pathogen 
colonization. Compatible plant pathogens then in turn evolved strategies such as the secretion of effectors, to avoid or suppress these mechanisms, and cause disease (Rovenich et al. 2014; Kombrink et al. 2017). Given that the mechanisms underlying basal defence are very diverse, it may very well be that the basal defence mechanisms in Moneymaker plants differ from those in VG-20. The QTLs for stunting that were contributed by Moneymaker may then contain genes involved in this basal defence. However, the severe fungal colonization and symptom expression of $V$. dahliae-infected Moneymaker plants, both found in this thesis and previous studies (i.e. Fradin et al. 2009; Yadeta 2012), illustrates that these two QTLs are not likely to provide a high level of resistance.

Compared to Moneymaker, VG-20 displayed clearly improved resistance against $V$. dahliae both in Chapter 5 of this thesis and in the work of Yadeta (2012). Therefore, the first focus should be on the validation of the three QTLs contributed by VG-20. Although all three QTLs from VG-20 were shown to reduce stunting symptoms, it is unclear whether all three QTLs are true resistance QTLs, and therefore also reduce the colonization of $V$. dahliae. The best approach to further validate the QTLs would be to develop near-isogenic lines (NILs) that are isogenic for most of the genome but possess the VG-20 allele for the QTL of interest. This way, the effect of each QTL on stunting and V. dahliae colonization can be tested in a fixed genetic background. Simultaneously, by comparing NILs that possess only one or multiple QTLs the VG-20 allele, it can be validated whether only one or multiple alleles of these QTLs are required to reach the highest level of resistance. Finally, by challenging these NILs with multiple V. dahliae strains, it can be determined how each individual QTL contributes to the broad-spectrum efficacy of the resistance of VG-20. Finally, our analysis also indicates that these three QTLs from VG-20 may not fully account for the resistance of VG-20 (Chapter 5). To identify the remaining resistance loci, a more powerful QTL analysis is probably required that is based on more RILs. Still, it is unknown how many additional causal genes underly the quantitative resistance, and whether all these genes can practically be used in breeding programs. It may therefore be best to first validate the QTLs that were already identified and assess whether a satisfactory level of resistance can be established with these QTLs.
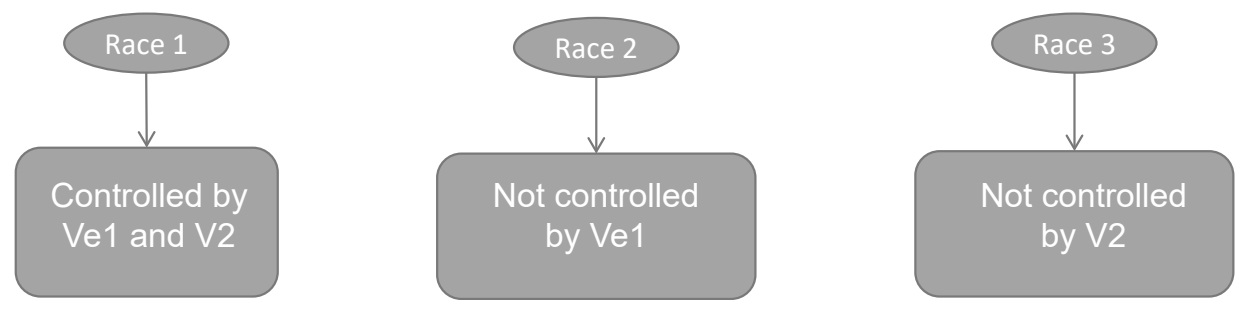

FICURE 1 | The latest classification of $V$. dahliae into race 1,2 and 3, based on the resistance genes Ve1 (Fradin et al. 2009; Kawchuk et al. 2001) and the recently described V2 locus (Usami et al. 2017). 


\section{Yellowing of leaves: another V. dahliae-related symptom?}

Strikingly, besides the segregation for $V$. dahliae stunting symptoms, a clear segregation was observed for yellowing of the lower leaves of $V$. dahliae inoculated RILs in the VG-3 RIL population (Chapter 4). This was surprising, since both Moneymaker and VG-3 did not display apparent foliar symptoms on all plants when inoculated with $V$. dahliae (Chapter 3). Since in the VG-20 and VG-21 RIL populations, yellowing, necrosis and intumescence developed on leaves of both mock-inoculated and $V$. dahliae-inoculated plants, $V$. dahliaeassociated yellowing symptoms could not be reliably scored (Fig. 1). This may raise the question whether the yellowing symptoms in the VG-3 RIL population are truly symptoms for $V$. dahliae infection. However, since in the VG-3 population, yellowing symptoms were not apparent on most mock-inoculated plants, and there was no meaningful correlation $\left(R^{2}=0.05\right)$ between the number of yellowing leaves in mock-inoculated and $V$. dahliaeinoculated plants, this indicates that the yellowing of the leaves in the VG-3 population was caused by the $V$. dahliae infection.

Intriguingly, the VG-3 RIL population displayed a vastly different segregation pattern for the stunting symptoms than for the yellowing symptoms. This indicates that different genetic mechanisms underly the segregation for stunting and the segregation for yellowing. Possibly, these different genetic mechanisms are active during different phases of the disease cycle. After the initial root penetration by $V$. dahliae, it can take the fungus up to a week to spread and accumulate in the stem xylem vessels (Fradin and Thomma 2006). Based on the disease trials with $V$. dahliae on tomato plants in this $\mathrm{PhD}$ research and previous trials (Fradin et al. 2009; Yadeta 2012), we know that stunting symptoms generally develop when the first true leaves begin to expand, at approximately 10 days after the inoculation. When the fungus enters its saprophytic stage, it starts to colonize the roots and shoots (Fradin and Thomma 2006). In this phase, senescence of leaves starts, which includes yellowing and necrosis of the leaves (Fradin and Thomma 2006). Based on our observations, yellowing symptoms generally seem to appear 2,5 weeks after the inoculation but become especially apparent from 3-4 weeks after the inoculation onwards. Likely, the genetic mechanisms that reduce stunting symptoms are therefore already activated in the early, pre-saprophytic phase of the disease cycle, whereas the genetic mechanisms that reduce yellowing symptoms may only be involved later, in the saprophytic phase of the disease cycle. Whereas Moneymaker plants display extensive fungal colonization and stunting when challenged with $V$. dahliae, Moneymaker plants only developed mild yellowing symptoms. This phenotype may be explained by the two QTLs that were identified in Chapter 4 , as these two QTLs from Moneymaker were found to reduce the number of yellowing leaves upon inoculation with $V$. dahliae. Since infected Moneymaker plants generally displayed more $V$. dahliae biomass accumulation, but not more severe yellowing symptoms than VG-3 plants (Chapter 3), these QTLs most likely govern tolerance to $V$. dahliae. This potential tolerance of 
Moneymaker to $V$. dahliae may then also be an explanation for why other studies mentioned more severe foliar symptoms on other susceptible tomato plants (i.e. Usami et al. 2017; Diwan et al. 1999). Simultaneously, it would explain why in these studies foliar symptoms were successfully used to distinguish resistance levels between plants, whereas in my PhD research the difference in yellowing symptoms seems not always to be associated with a difference in $V$. dahliae colonization.

\section{Verticillium dahliae race 3: the arms-race continues}

For over 65 years, the only reported qualitative $R$ gene against $V$. dahliae was $V e 1$, which is only effective against race 1 strains that possess the $V d A v e 1$ effector and not against race 2 strains that lack VdAve1. Fortunately, in recent years, several additional resistances have been reported in tomato, including the three wild accessions studied in my $\mathrm{PhD}$ research. In 2017, a resistant tomato cultivar named Aibou was identified by Usami et al. (2017) which possesses a dominantly inherited locus named V2. Although V2 provides a relatively high level of resistance, several $V$. dahliae strains have overcome this resistance. This finding warranted a re-classification of $V$. dahliae races. The latest classification divides $V$. dahliae strains into three races: Strains which have not overcome the resistance of $V e 1$ and $V 2$ are assigned to race 1 , whereas strains which have overcome the resistance of Ve1 or V2 are assigned to race 2 or 3 , respectively (Fig. 2). Taking this into consideration, a sensible step in $V$. dahliae resistance breeding would be to combine the resistance of Ver and V2, as this would provide a broader resistance spectrum. However, currently it is not clear to what extent race 3 strains are prevalent worldwide, and thereby to what extent Ver and V2 resistant plants can effectively be used to control $V$. dahliae.

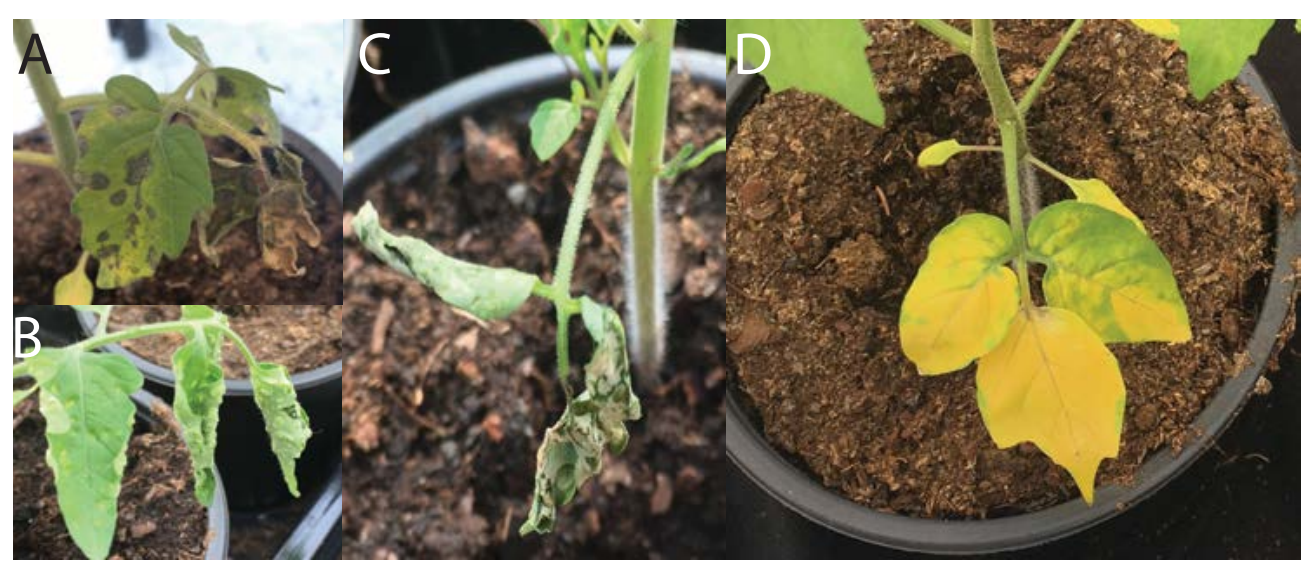

FICURE 2 | Examples of necrosis (A), intumescence (B-C) and yellowing of leaves of mock-inoculated leaves of the Moneymaker x VG-20 (A-D) and Moneymaker x VG-21 (B-C) RIL populations. 
In Chapter 6 we pursued the hypothesis that, like Ve1, the potential immune receptor encoded by V2 detects a race 2-specific avirulence effector, "Av2", which is not present in race 3 strains. Based on a comparative genomics analysis with a selection of race 2 and 3 strains we identified a $277 \mathrm{~kb}$ race 2 -specific genomic sequence which is absent in race 3 strains. Only two effector candidates in this region are expressed in planta, making it very likely that one of these candidates is the Av2 avirulence factor. Assuming that further analyses will confirm that indeed one of these candidates is Av2, this information is of value for the future management of Verticillium wilt disease on tomato. First of all, if Av2 triggers a robust hypersensitive response on V2 plants, Av2 can be used as a probe to locate the V2 gene. After all, if the heritability of a $V 2$-triggered hypersensitive response is better than the $V$. dahliae induced stunting symptoms, it may make the mapping and introgression of $V 2$ easier. Secondly, the presence of both effector candidates gives insights in the prevalence of race 3 strains. Among the strains which lack both avirulence effector candidates are strains originally isolated from Canada, the USA, China, the Netherlands and Japan. Although additional analyses need to further unravel how widespread race 3 strains are these countries, resistance governed by Ve1 and V2 may thus not always be effective to control Verticillium wilt. Furthermore, worldwide employment of Ve1 and V2 resistant plants will inevitably cause a selection pressure on $V$. dahliae strains to lose, or modify, the Av2 gene. As, based on our pathogenicity assays with race 2 and 3 strains, no apparent difference in virulence was detected, the virulence contribution of Av2 is probably modest. This suggests that, unlike the VdAver effector (de Jonge et al. 2012), there may be no severe virulence cost for race 2 strains to lose Av2. This suggests that it may be relatively easy for race 2 strains to evade detection by $\mathrm{V} 2$. In any case, also to control race 3 strains, additional resistances are thus still required to control V. dahliae. Fortunately, the three QTLs from VG-20 that were identified in Chapter 5 may already provide resistance against race 3 of $V$. dahliae, as the strain $V$. dahliae DVDS29 that was used for the QTL analyses belongs to race 3 as it lacks both candidate effector genes. Validation and introgression of these QTLs could thus be a promising strategy to further control race 3 of $V$. dahliae.

\section{The apparent rarity of $\mathrm{R}$ genes against $\mathrm{V}$. dahliae}

Thus far, qualitative $R$ gene-based resistance have been the main source of resistance in the generation of $V$. dahliae-resistant tomato plants, first with the introduction of $V e I$ in crops in the 1950s (Deseret News and Telegram 1955), and since 2006 with the introduction of Ve1- and V2 resistant Aibou plants in Japan (Usami et al. 2017). However, these two $R$ genes against $V$. dahliae in tomato seem to be the exception. Although some crops possess homologs of VeI (Song et al. 2016), V. dahliae resistance screens in other crops such as cotton, strawberry, eggplant and rapeseed typically report quantitative resistance against V. dahliae, based on multiple QTLs (Rygulla et al. 2008; Antanaviciute et al. 2015; Bolek et 
al. 2005; Wang et al. 2008; Zhao et al. 2014; Toppino and Barchi 2016). When compared with other vascular pathogens, for instance Fusarium oxysporum, $R$ genes against $V$. dahliae thus seem to be rather rare. After all, at least $18 R$ genes have been described against $F$. oxysporum, of which nine in tomato only (de Sain and Rep 2015). Given the similar infection cycle and nearly identical symptoms of F. oxysporum and $V$. dahliae on tomato, this apparent difference in prevalence of $R$ genes is remarkable.

However, several differences in the biology of the two pathogens may explain why $R$ genes against $F$. oxysporum appear to be far more prevalent than $R$ genes against $V$. dahliae. First of all, although $F$. oxysporum and $V$. dahliae both have a wide host range, individual $V$. dahliae strains usually display a far broader host range than individual F. oxysporum strains. Typically, $V$. dahliae strains display a low degree of adaptation to individual host species. Although some $V$. dahliae effectors have been identified that are crucial pathogenicity factors on particular hosts (Li 2019), $V$. dahliae strains typically infect a wide array of host plants. In contrast, strains of F. oxysporum are grouped into formae speciales, which can generally only infect one or very few plant species (Edel-Hermann and Lecomte 2019). For example, tomato-infecting fusarium strains belong to F. oxysporumf. sp. lycopersici, whereas banana-infecting strains belong to F. oxysporum f. sp. cubense.

For a host species to evolve resistance, it needs to be exposed to a pathogen that impairs its fitness. After all, infection must be present for resistance against be advantageous, and thus be favoured by selection (Roy and Kirchner 2000; Antonovics et al. 2013). Consequently, the more a host plant is exposed to a particular elicitor, the higher the chance that the host evolves an $R$ gene encoding an immune receptor that recognizes this effector. One important class of elicitors that are recognized by $R$ genes are effectors, which are produced by the pathogen to support the colonization of its host (Rovenich et al. 2014). Of F. oxysporum, it has been determined that strains that infect the same host plant carry highly similar effector profiles, and that formae speciales can be distinguished based on these effector profiles (de Sain and Rep 2015). In contrast, $V$. dahliae strains that infect the same host plants carry highly divergent effector repertoires, and the host range does generally not correlate with the composition of these effector repertoires (Gibriel et al. 2019; Li 2019). Thus, when a plant immune receptor recognizes a $V$. dahliae effector, the $R$ gene that encodes this immune receptor will often only provide a minor fitness benefit, as it is likely that this particular effector is only present in a small proportion of $V$. dahliae strains. This minor fitness benefit will probably cause the spread of this $R$ gene through the host population to be limited. In contrast, when an $R$ gene recognizes a F. oxysporum effector, this effector is far more likely to be shared among most or all other F. oxysporum strains of the particular forma specialis, which therefore provides a greater fitness benefit. This may thus make the $R$ gene spread more extensively through the host population, increasing the chance that breeders or phytopathologists encounter this $R$ gene. Finally, also the distribution of the effectors 
inside the plants may have an influence on the evolution of $R$ gene resistance against $V$. dahliae. In contrast to F. oxysporum, little convincing evidence exists that $V$. dahliae employs effectors that reach the cytoplasm of hosts cells. Considering that one of the major classes of $R$-genes, NB-LRRs, resides in the cytoplasm, it is unlikely that this class of $R$ genes is involved in the resistance against $V$. dahliae in tomato.

\section{Future perspectives of resistance breeding against $V$. dahliae}

In an evolutionary sense, no resistance lasts forever. It is therefore inevitable that, sooner or later, additional resistances to $V$. dahliae will need to be identified and employed to further control this disease. My PhD research and the research of Yadeta (2012) has demonstrated that wild tomato germplasm can be a valuable pool for the identification of additional resistances against $V$. dahliae. However, my research also illustrates that the genetics underlying quantitative resistance against $V$. dahliae cannot always easily be unravelled. Fortunately, recent developments can facilitate scientists to identify quantitative resistance loci more effectively. More advanced mapping populations have been developed, such as genome-wide association mapping (GWA), nested association mapping (NAM) populations (McMullen et al. 2009) and multiparent advanced generation intercross (MAGIC) populations (Beyer et al. 2008), which have a greatly enhanced ability to dissect genetically complex traits. Simultaneously, automated phenotyping platforms allow researchers to screen larger numbers of plants, resulting in higher discriminating power in the mapping analyses. Although it remains to be seen whether highly polygenic resistance can practically be used in breeding programs, gaining an increased understanding of these resistance mechanisms may help to design novel strategies to control diseases such as Verticillium wilt. Furthermore, identification and introgression of only the QTLs with the largest contributions to the quantitative resistance, such as the QTLs for the resistance of VG-20 we mapped in Chapter 5, may still provide a sufficient level of resistance against control $V$. dahliae.

Despite the apparent rarity of $R$ genes against $V$. dahliae, strategies can be adapted to accelerate the identification of $R$ genes. Over the last decades, the decreasing costs of sequencing technologies has resulted in a rapid identification of $V$. dahliae effectors (de Jonge et al. 2012; Li 2019). These effectors can be valuable probes to detect $R$ genes in tomato against $V$. dahliae. This strategy was first adapted against Phytophtora infestans, and it has led to an accelerated cloning of $R$ genes against this pathogen (Vleeshouwers et al. 2011; Vleeshouwers and Oliver 2014). Probing of wild tomato germplasm collections with $V$. dahliae effectors may thus also accelerate the identification of $R$ genes against $V$. dahliae. Furthermore, selection of effectors which are important for the virulence or pathogenicity of the fungus may potentially provide more durable resistance against $V$. dahliae. For 
example, a single effector, Tom1, was recently found to be crucial for the pathogenicity of $V$. dahliae on tomato (Li 2019). If an $R$ gene could be detected which encodes an immune receptor that recognizes this effector, it probably will provide durable resistance against $V$. dahliae, as the pathogen cannot afford to lose this Tom1 effector. Simultaneously, it may be of interest to study the interaction with Tom1 with host tomato plants. Although effectors are mostly known for their suppression of host resistance, many effectors may in fact activate susceptibility $(S)$ genes of the host. Identification and subsequent impairment of these $S$ genes may therefore be a promising alternative strategy to achieve resistance against V. dahliae (van Schie and Takken 2014).

Overall, with the right tools and scientific knowledge, both quantitative resistance and qualitative resistance can thus successfully be identified and employed in plant breeding programs. In the end, to achieve durable resistance against $V$. dahliae, an interesting approach would be to combine the potential broad-spectrum, partial resistance QTLS identified in this thesis with the more narrow-spectrum but stronger resistance governed by Ve1 and V2 (Pilet-Nayel et al. 2017). 
References 



\section{References}

Acquaah, G. 2012. Principles of plant genetics and breeding. Wiley-Blackwell.

Alexander, D., Goodman, R. M., Gut-Rella, M., Glascock, C., Weymann, K., Friedrich, L., Maddox, D., Ahl-Goy, P., Luntz, T., Ward, E., Ryals, J. 1993. Increased tolerance to two oomycete pathogens in transgenic tobacco expressing pathogenesis-related protein 1a. Proc. Natl. Acad. Sci. U. S. A. 90:7327-7331

Alexander, L. J. 1962. Susceptibility of certain Verticillium-resistant tomato varieties to an ohio isolate of the pathogen. Phytopathology. 53:998-1000

Allard, R. W. 1960. Principles of plant breeding. Wiley.

Antanaviciute, L., Surbanovski, N., Harrison, N., McLeary, K. J., Simpson, D. W., Wilson, F., Sargent, D. J., and Harrison, R. J. 2015. Mapping QTL associated with Verticillium dahliae resistance in the cultivated strawberry (Fragaria ananassa). Hortic. Res. 2:1-8

Antoniou, P. P., Markakis, E. A., Tjamos, S. E., Paplomatas, E. J., and Tjamos, E. C. 2008. Novel methodologies in screening and selecting olive varieties and root-stocks for resistance against Verticillium dahliae. Eur. J. Plant Pathol. 122:549-560

Antonovics, J., Boots, M., Ebert, D., Koskella, B., Poss, M., and Sadd, B. M. 2013. The origin of specificity by means of natural selection: Evolved and nonhost resistance in hostpathogen interactions. Evolution. 67:1-9

Appiano, M. 2016. PhD Thesis, Wageningen University. Insights into the mlo-based powdery mildew resistance.

Asamizu, E., and Ezura, H. 2009. Inclusion of tomato in the genus Solanum as "Solarium lycopersicum" is evident from phylogenetic studies. J. Japanese Soc. Hortic. Sci. 78:3-5

Ashrafi, H., Kinkade, M., and Foolad, M. R. 2009. A new genetic linkage map of tomato based on a Solanum lycopersicum $\times$ S. pimpinellifolium RIL population displaying locations of candidate pathogen response genes. Genome. 52:935-956

Baack, E. J., and Rieseberg, L. H. 2007. A genomic view of introgression and hybrid speciation. Curr. Opin. Genet. Dev. 17:513-518
Baergen, K. D. D., Hewitt, J. D. D., St. Clair, D. A., and Clair, D. A. S. 1993. Resistance of tomato genotypes to four isolates of Verticillium dahliae race 2. HortScience. 28:833-836

Bai, Y., and Lindhout, P. 2007. Domestication and breeding of tomatoes: what have we gained and what can we gain in the future? Ann. Bot. 100:1085-1094

Bai, Y., and Lindhout, P. 2008. New challenges for durable resistance breeding in tomato. Acta Hortic. 789:61-73

Ballini, E., More, J. B., Droc, G., Price, A., Courtois, B., Notteghem, J. L., and Tharreau, D. 2008. A genome-wide meta-analysis of rice blast resistance genes and quantitative trait loci provides new insights into partial and complete resistance. Mol. Plant-Microbe Interact. 21:859-868

Bauchet, G., and Causse, M. 2012. Genetic diversity in tomato (Solanum lycopersicum) and its wild relatives. Genet. Divers. Plants. p133-162. ISBN 978-953-51-0185-7

Beavis, W. D. 1998. QTL analyses: Power, precision, and accuracy. Pages 145-162 in: Molecular Dissection of Complex Traits

Belcher, A. R., Zwonitzer, J. C., Cruz, J. S., Krakowsky, M. D., Chung, C. L., Nelson, R., Arellano, C., and Balint-Kurti, P. J. 2012. Analysis of quantitative disease resistance against southern leaf blight and of multiple disease resistance in maize, using near-isogenic lines. Theor. Appl. Genet. 124:433-445

Bender, C. G. G., and Shoemaker, P. B. B. 1984. Prevalence of Verticillium wilt of tomato and virulence of Verticillium dahliae race 1 and race 2 isolates in Western North cCrolina. Plant Dis. 68:305-309

Bergougnoux, V. 2014. The history of tomato: From domestication to biopharming. Biotechnol. Adv. 32:170-189

Bertels, F., Silander, O. K., Pachkov, M., Rainey, P. B., and Van Nimwegen, E. 2014. Automated reconstruction of whole-genome phylogenies from short-sequence reads. Mol. Biol. Evol. 31:1077-1088 
Beyer, P., Morell, M., Mackay, I., and Powell, W. 2008. From mutations to MAGIC: resources for gene discovery, validation and delivery in crop plants. Curr. Opin. Plant Biol. 11:215-221

van der Biezen, E. A., and Jones, J. D. G. 1998. Plant disease-resistance proteins and the gene-forgene concept. Trends Biochem. Sci. 23:454-456

Bishop, C. D., and Cooper, R. M. 1983. An ultrastructural study of root invasion in three vascular wilt diseases. Physiol. Plant Pathol. 22:15-IN13

Blanca, J., Cañizares, J., Cordero, L., Pascual, L., Diez, M. J., and Nuez, F. 2012. Variation revealed by SNP genotyping and morphology provides insight into the origin of the tomato. PLoS One. 7

Bletsos, F., Thanassoulopoulos, C., and Roupakias, D. 2003. Effect of grafting on growth, yield, and Verticillium wilt of eggplant. HortScience. 38:183-186

Block, A., Schmelz, E., O'Donnell, P. J., Jones, J. B., and Klee, H. J. 2005. Systemic acquired tolerance to virulent bacterial pathogens in tomato. Plant Physiol. 138:1481-1490

Bolek, Y., El-Zik, K. M., Pepper, A. E., Bell, A. A., Magill, C. W., Thaxton, P. M., and Reddy, O. U. K. 2005. Mapping of Verticillium wilt resistance genes in cotton. Plant Sci. 168:1581-1590

Boller, T., and Felix, G. 2009. A renaissance of elicitors: perception of microbe-associated molecular patterns and danger signals by patternrecognition receptors. Annu. Rev. Plant Biol. 60:379-406

Boshoven, J. C. 2017. PhD Thesis, Wageningen University. Virulence contribution and recognition of homologs of the Verticillium dahliae effector Ave1.

Bradley, D. J., Gilbert, G. S., and Parker, I. M. 2003. Susceptibility of clover species to fungal infection: The interaction of leaf surface traits and environment. Am. J. Bot. 90:857-864

Broman, K. W. 2010. Genetic map construction with R/ qtl. http://www.rqtl.org

Broman, K. W., and Sen, S. 2009. A Guide to QTL Mapping with R/qtl. Springer US.

Broman, K. W., Wu, H., Sen, Ś., and Churchill, G. A. 2003. R/qtl: QTL mapping in experimental crosses. Bioinformatics. 19:889-890

Brown, J. K. M. 2003. A cost of disease resistance: paradigm or peculiarity? Trends Genet. 19:667671
Brown, J. K. M. 2015. Durable resistance of crops to disease: a Darwinian perspective. Annu. Rev. Phytopathol. 53:513-539

Brown, J. K. M. 2002. Yield penalties of disease resistance in crops. Curr. Opin. Plant Biol. 5:339-344

Buchner, V., Burstein, Y., and Nachmias, A. 1989. Comparison of Verticillium dahliae-produced phytotoxic peptides purified from culture fluids and infected potato stems. Physiol. Mol. Plant Pathol. 35:253-269

Buell, C. R., and Somerville, S. C. 1995. Expression of defence-related and putative signaling genes during tolerant and susceptible interactions of Arabidopsis with Xanthomonas campestris pv. campestris. Mol. Plant-Microbe Interact. 8:435-443

Buhtz, A., Witzel, K., Strehmel, N., Ziegler, J., Abel, S., and Grosch, R. 2015. Perturbations in the primary metabolism of tomato and Arabidopsis thaliana plants infected with the soil-borne fungus Verticillium dahliae. PLoS One. 10:1-14

Busch, L. V., and Smith, E. 1981. Susceptibility of Ontario-grown alfalfa cultivars and certain Medicago species to Verticillium albo-atrum. Can. J. Plant Pathol. 3:169-172

Chaloner, T., van Kan, J. A. L., and Grant-Downton, R. T. 2016. RNA 'Information warfare' in pathogenic and mutualistic interactions. Trends Plant Sci. 21:738-748

Chamnongpol, S., Willekens, H., Moeder, W., Langebartels, C., Sandermann, H., Van Montagu, M., Inzé, D., and Van Camp, W. 1998. Defence activation and enhanced pathogen tolerance induced by $\mathrm{H}_{2} \mathrm{O}_{2}$ in transgenic tobacco. Proc. Natl. Acad. Sci. U. S. A. 95:58185823

Chen, F., He, G., He, H., Chen, W., Zhu, X., Liang, M., Chen, L., and Deng, X. W. 2010. Expression analysis of miRNAs and highly-expressed small RNAs in two rice subspecies and their reciprocal hybrids. J. Integr. Plant Biol. 52:971-980

Chen, P., Lee, B., and Robb, J. 2004. Tolerance to a non-host isolate of Verticillium dahliae in tomato. Physiol. Mol. Plant Pathol. 64:283-291

Cirulli, M. 1969. Un isolato di Verticillium dahliae Kleb. virulento verso varietà resistenti di Pomodoro. Phytopathol. Mediterr. 8:132-136 
Clint Nesbitt, T., and Tanksley, S. D. 2002. Comparative sequencing in the genus Lycopersicon: Implications for the evolution of fruit size in the domestication of cultivated tomatoes. Genetics. 162:365-379

Collard, B. C. Y., Jahufer, M. Z. Z., Brouwer, J. B., and Pang, E. C. K. 2005. An introduction to markers, quantitative trait loci (QTL) mapping and marker-assisted selection for crop improvement: The basic concepts. Euphytica. 142:169-196

Cook, D. E., Mesarich, C. H., and Thomma, B. P. H. J. 2015. Understanding plant immunity as a surveillance system to detect invasion. Annu. Rev. Phytopathol. 53:541-563

Cooper, J., and Dobson, H. 2007. The benefits of pesticides to mankind and the environment. Crop Prot. 26:1337-1348

Corwin, J. A., and Kliebenstein, D. J. 2017. Quantitative resistance: More than just perception of a pathogen. Plant Cell. 29:655-665

Cowger, C., and Brown, J. K. M. 2019. Durability of quantitative resistance in crops: greater than we know? Annu. Rev. Phytopathol. 57:253-277

Crick, F., and Watson, J. 1953. Molecular structure of nucleic acids: a structure for deoxyribose nucleic acid. Nature. 171:737-738

Crisp, P. A., Hammond, R., Zhou, P., Vaillancourt, B., Lipzen, A. M., Daum, C., Barry, K. W., de Leon, N., Buell, C. R., Kaeppler, S. M., Meyers, B. C., Hirsch, C., and Springer, N. M. 2019. Variation and inheritance of small RNAs in maize inbreds and Fi hybrids. Plant Physiol. 182: 318-331

Dan, H., Ali-Khan, S. T., and Robb, J. 2001 . Use of quantitative PCR diagnostics to identify tolerance and resistance against Verticillium dahliae in potato. Plant Dis. 85:700-705

Dangl, J. L., Horvath, D. M., and Staskawich, B. J. 2013. Pivoting the plant immune system. Science. 341:745-751

Dangl, J. L., and Jones, J. D. G. 2001. Plant pathogens and integrated defence responses to infection. Nature. 411:826-833

Darvasi, A., Weinreb, A., Minke, V., Weller, J. I., and Soller, M. 1993. Detecting marker-QTL linkage and estimating qtl gene effect and map location using a saturated genetic map. Genetics. 134:943-951
Darvill, A. G., and Albersheim, P. 1984. Phytoalexins and their elicitors-a defence against microbial infection in plants. Annu. Rev. Plant Physiol. 35:243-275

Davar, R., Darvishzadeh, R., and Majd, A. 2011. Genotype-isolate interaction for resistance against Sclerotinia sclerotiorum in sunflower. Phytopathol. Mediterr. 50:442-449

Dean, R. A., Lichens-Park, A., and Kole, C. 2014. Genomics of plant-associated fungi and oomycetes: Dicot pathogens.

Depotter, J. R. L., Thomma, B. P. H. J., and Wood, T. A. 2019. Measuring the impact of Verticillium longisporum on oilseed rape (Brassica napus) yield in field trials in the United Kingdom. Eur. J. Plant Pathol. 153:321-326

Deseret News and Telegram. Salt Lake City, Wednesday 14 december 1955. From Jungles Of Peru.

Diwan, N., Fluhr, R., Eshed, Y., Zamir, D., and Tanksley, S. D. 1999. Mapping of Ve in tomato: a gene conferring resistance against the broadspectrum pathogen, Verticillium dahliae race 1. Theor. Appl. Genet. 98:315-319

Dobinson, K. F., Tenuta, G. K. K., and Lazarovits, G. 1996. Occurence of race 2 of Verticillium dahliae in processing tomato fields in southwestern ontario. Can. J. Plant Pathol. 18:55-58

Dodds, P. N., and Rathjen, J. P. 2010. Plant immunity: towards an integrated view of plant-pathogen interactions. Nat. Rev. Genet. 11:539-48

Dong, S., Raffaele, S., and Kamoun, S. 2015. The twospeed genomes of filamentous pathogens: Waltz with plants. Curr. Opin. Genet. Dev. 35:57-65

Du, J., Verzaux, E., Chaparro-Garcia, A., Bijsterbosch, G., Keizer, L. C. P., Zhou, J., Liebrand, T. W. H., Xie, C., Govers, F., Robatzek, S., Van Der Vossen, E. A. G., Jacobsen, E., Visser, R. G. F., Kamoun, S., and Vleeshouwers, V. G. A. A. 2015. Elicitin recognition confers enhanced resistance against Phytophthora infestans in potato. Nat. Plants. 1:1-5

Edel-Hermann, V., and Lecomte, C. 2019. Current status of Fusarium oxysporum formae speciales and races. Phytopathology. 109:512-530

van Esse, H. P., Thomma, B. P. H. J., van 'T Klooster, J. W., and de Wit, P. J. G. M. 2006. Affinity-tags are removed from Cladosporium fulvum effector proteins expressed in the tomato leaf apoplast. J. Exp. Bot. 57:599-608 
Essenbeck, N. von. 1817. Das System der Pilze und Schwämme. Würzburg.

Faino, L., Seidl, M. F., Datema, E., van den Berg, G. C. M., Janssen, A., Wittenberg, A. H. J., and Thomma, B. P. H. J. 2015. Single-molecule realtime sequencing combined with optical mapping yields completely finished fungal genome. MBio. 6:1-11

Faino, L., Seidl, M. F., Shi-Kunne, X., Pauper, M., van den, G. C., Wittenberg, A. H., and Thomma, B. P. H. J. 2016. Transposons passively and actively contribute to evolution of the two-speed genome of a fungal pathogen. Genome Res. 26:1091-1100

Fan, R., Cockerton, H. M., Armitage, A. D., Bates, H., Cascant-Lopez, E., Antanaviciute, L., Xu, X., Hu, X., and Harrison, R. J. 2018. Vegetative compatibility groups partition variation in the virulence of Verticillium dahliae on strawberry. PLoS One. 13:1-21

Fang, H., Zhou, H., Sanogo, S., Flynn, R., Percy, R. G., Hughs, S. E., Ulloa, M., Jones, D. C., and Zhang, J. 2013. Quantitative trait locus mapping for Verticillium wilt resistance in a backcross inbred line population of cotton (Gossypium hirsutum $\times$ Gossypium barbadense) based on RGA-AFLP analysis. Euphytica. 194:79-91

Fang, H., Zhou, H., Sanogo, S., Lipka, A. E., Fang, D. D., Percy, R. G., Hughs, S. E., Jones, D. C., Gore, M. A., and Zhang, J. 2014. Quantitative trait locus analysis of Verticillium wilt resistance in an introgressed recombinant inbred population of Upland cotton. Mol. Breed. 33:709-720

FAOSTAT. 2020. FAOSTAT. Available at: http://www. fao.org/faostat/en/\#data [Accessed January 19, 2020].

Flood, J. 2010. The importance of plant health to food security. Food Secur. 2:215-231

Flor, H. H. 1942. Inheritance of pathogenicity in Melampsora lini. Phytopathology. 32:653-669

Focus, N. 2007. Seeking agriculture's ancient roots. Science. 316:1830-1835

Fradin, E. F., and Thomma, B. P. H. J. 2006. Physiology and molecular aspects of Verticillium wilt diseases caused by V. dahliae and V. albo-atrum. Mol. Plant Pathol. 7:71-86

Fradin, E. F., Zhang, Z., Juarez Ayala, J. C., Castroverde, C. D. M., Nazar, R. N., Robb, J., Liu, C.-M., and Thomma, B. P. H. J. 2009. Genetic dissection of Verticillium wilt resistance mediated by tomato Ve1. Plant Physiol. 150:320332
Fradin, E. F., Zhang, Z., Rovenich, H., Song, Y., Liebrand, T. W. H., Masini, L., Van Den Berg, G. C. M., Joosten, M. H. A. J., and Thomma, B. P. H. J. 2014. Functional analysis of the tomato immune receptor Ve1 through domain swaps with its non-functional homolog Ve2. PLoS One. 9

Gibriel, H. A. Y., Zhu, L. J., Seidl, M. F., and Thomma, B. P. H. J. 2019. Verticillium dahliae strains that infect the same host plant display highly divergent effector catalogs. BioRxiv.

Gold, J., and Robb, J. 1995. The role of the coating response in craigella tomatoes infected with Verticillium dahliae, races 1 and 2. Physiol. Mol. Plant Pathol. 47:141-157

Gould, S. B., Waller, R. F., and McFadden, G. I. 2008. Plastid Evolution. Annu. Rev. Plant Biol. 59:491-517

Grayston, S. J., Vaughan, D., and Jones, D. 1997. Rhizosphere carbon flow in trees, in comparison with annual plants: The importance of root exudation and its impact on microbial activity and nutrient availability. Appl. Soil Ecol. 5:29-56

Grogan, R. G. 1979. Verticillium wilt on resistant tomato cultivars in california: virulence of isolates from plants and soil and relationship of inoculum density to disease incidence. Phytopathology. 69:1176

Groszmann, M., Greaves, I. K., Albertyn, Z. I., Scofield, G. N., Peacock, W. J., and Dennis, E. S. 2011. Changes in 24-nt siRNA levels in Arabidopsis hybrids suggest an epigenetic contribution to hybrid vigor. Proc. Natl. Acad. Sci. U. S. A. 108:2617-2622

Hajjar, R., and Hodgkin, T. 2007. The use of wild relatives in crop improvement: A survey of developments over the last 20 years. Euphytica. 156:1-13

Han, G. 2019. Origin and evolution of the plant immune system. New Phytol. 222:70-83

Hansen, H. P. 1934. Inheritance of resistance against plant diseases caused by fungi, bacteria and vira. Pages 1-74 in: Yearbook of the Royal Veterinary and Agricultural College, Copenhagen, Denmark,

Hily, J. M., Poulicard, N., Mora, M. Á., Pagán, I., and García-Arenal, F. 2016. Environment and host genotype determine the outcome of a plant-virus interaction: From antagonism to mutualism. New Phytol. 209:812-822 
Hollomon, D. W. 2015. Fungicide Resistance in Plant Pathogens. Fungic. Resist. Plant Pathog. p. 3-11

Huisman, O. C. 1988. Seasonal colonization of roots of field-grown cotton by verticillium dahliae and $\mathrm{v}$. tricorpus. Phytopathology. 78:708

Humplík, J. F., Lazár, D., Husičková, A., and Spíchal, L. 2015. Automated phenotyping of plant shoots using imaging methods for analysis of plant stress responses - A review. Plant Methods. 11:1-10

Inderbitzin, P., Bostock, R. M., Davis, R. M., Usami, T., Platt, H. W., and Subbarao, K. V. 2011. Phylogenetics and taxonomy of the fungal vascular wilt pathogen Verticillium, with the descriptions of five new species. PLoS One. 6

Inglese, S. J., and Paul, N. D. 2006. Tolerance of Senecio vulgaris to infection and disease caused by native and alien rust fungi. Phytopathology. 96:718-726

Jiang, F., Zhao, J., Zhou, L., Guo, W. Z., and Zhang, T. Z. 2009. Molecular mapping of Verticillium wilt resistance QTL clustered on chromosomes D7 anD D9 in upland cotton. Sci. China, Ser. C Life Sci. 52:872-884

Jiménez-Díaz, R. M., Olivares-García, C., TraperoCasas, J. L., Jiménez-Gasco, M. M., NavasCortés, J. A., Landa, B. B., and Milgroom, M. G. 2017. Variation of pathotypes and races and their correlations with clonal lineages in Verticillium dahliae. Plant Pathol. 66:651-666

Johnson, R. 1981. Durable resistance: definition of, genetic control, and attainment in plant breeding. Phytopathology. 71:567

Jones, J. B. 1991. Compendium of tomato diseases. American Phytopathological Society. ISBN 9780890544242

Jones, J. D. G. J. D. G., and Dangl, J. L. J. L. 2006. The plant immune system. Nature. 444:323-9

De Jonge, R., Bolton, M. D., Kombrink, A., Van Den Berg, G. C. M., Yadeta, K. A., and Thomma, B. P. H. J. 2013. Extensive chromosomal reshuffling drives evolution of virulence in an asexual pathogen. Genome Res. 23:1271-1282

de Jonge, R., Bolton, M. D., and Thomma, B. P. H. J. 2011. How filamentous pathogens co-opt plants: The ins and outs of fungal effectors. Curr. Opin. Plant Biol. 14:400-406

de Jonge, R., van Esse, H. P., Maruthachalam, K., Bolton, M. D., Santhanam, P., Saber, M. K., Zhang, Z., Usami, T., Lievens, B., Subbarao,
K. V, and Thomma, B. P. H. J. 2012. Tomato immune receptor Ve1 recognizes effector of multiple fungal pathogens uncovered by genome and RNA sequencing. Proc. Natl. Acad. Sci. U. S. A. 109:5110-5

Juvik, J. A., Bolkan, H., and Tanksley, S. D. 1991. The Ve gene for race 1 Verticillium resistance is on chromosome 7. Tomato Genet. Coop. 41:23 van Kan, J. A., van den Ackerveken, G. F., and de Wit, P. J. 1991. Cloning and characterization of $\mathrm{cDNA}$ of avirulence gene avry of the fungal pathogen Cladosporium fulvum, causal agent of tomato leaf mold. Mol. Plant. Microbe. Interact. 4:52-59

Kang, H., Wang, Y., Peng, S., Zhang, Y., Xiao, Y., Wang, D., Qu, S., Li, Z., Yan, S., Wang, Z., Liu, W., Ning, Y., Korniliev, P., Leung, H., Mezey, J., McCouch, S. R., and Wang, G. L. 2016. Dissection of the genetic architecture of rice resistance against the blast fungus Magnaporthe oryzae. Mol. Plant Pathol. 17:959-972

Kawchuk, L. M., Hachey, J., Lynch, D. R., Kulcsar, F., van Rooijen, G., Waterer, D. R., Robertson, a, Kokko, E., Byers, R., Howard, R. J., Fischer, R., and Prufer, D. 2001. Tomato Ve disease resistance genes encode cell surface-like receptors. Proc. Natl. Acad. Sci. U. S. A. 98:6511-5

Kerr, E. A., and Busch, L. V. 1977. A suggestion of linkage between Ve and alb. Tomato Genet. Coop. 27:18

Kerr, E. A., and Busch, L. V. 1975. Test of Ve, Verticillium resistance, with genes on chromosome 4 . Tomato Genet. Coop. 25:10

Klebahn, H. 1913. Beitrage zur Kenntnis der fungi imperfecti. I. eine Verticillium-krankheit auf dahlien. Mycol. Cent. :49-66

Klosterman, S. J., Atallah, Z. K., Vallad, G. E., and Subbarao, K. V. 2009. Diversity, pathogenicity, and management of Verticillium species. Annu. Rev. Phytopathol. 47:39-62

Kombrink, A., Rovenich, H., Shi-Kunne, X., RojasPadilla, E., van den Berg, G. C. M., Domazakis, E., de Jonge, R., Valkenburg, D. J., Sanchez-Vallet, A., Seidl, M. F., and Thomma, B. P. H. J. 2017. Verticillium dahliae LysM effectors differentially contribute to virulence on plant hosts. Mol. Plant Pathol. 18:596-608

Koren, S., Walenz, B. P., Berlin, K., Miller, J. R., Bergman, N. H., and Phillippy, A. M. 2017. Canu: scalable and accurate long-read assembly via adaptive k-mer weighting and repeat separation. Genome Res. 27:722-736 
Kourelis, J., and Van Der Hoorn, R. A. L. 2018. Defended to the nines: 25 years of resistance gene cloning identifies nine mechanisms for $\mathrm{R}$ protein function. Plant Cell. 30:285-299

Kubota, C., Eguchi, T., and Kroggel, M. 2017. UV-B radiation dose requirement for suppressing intumescence injury on tomato plants. Sci. Hortic. (Amsterdam). 226:366-371

Kump, K. L., Bradbury, P. J., Wisser, R. J., Buckler, E. S., Belcher, A. R., Oropeza-Rosas, M. A., Zwonitzer, J. C., Kresovich, S., McMullen, M. D., Ware, D., Balint-Kurti, P. J., and Holland, J. B. 2011. Genome-wide association study of quantitative resistance against southern leaf blight in the maize nested association mapping population. Nat. Genet. 43:163-168

Lamichhane, J. R., Dachbrodt-Saaydeh, S., Kudsk, P., and Messéan, A. 2016. Conventional Pesticides in Agriculture: Benefits Versus Risks. Plant Dis. 100:10-24

Lander, E. S., and Botstein, D. 1989. Mapping mendelian factors underlying quantitative traits using rflp linkage maps. Genetics. 121:185-199

Lang, S., and Tibbitts, T. 1983. Factors controlling intumescence development on tomato plants. J. Am. Soc. Hortic. Sci. 108:93-98

Langmead, B., and Salzberg, S. L. 2012. Fast gappedread alignment with Bowtie 2. Nat. Methods. 9:357-359

Latterot, H. 1984. Specific resistance of Verticillium dahlias race 2 in tomato. Tomato Genet. Coop. 34:10-11

Lauge, R., Joosten, M. H. A. J., Haanstra, J. P. W., Goodwin, P. H., Lindhout, P., and de Wit, P. J. G. M. 1998. Successful search for a resistance gene in tomato targeted against a virulence factor of a fungal pathogen. Plant Biol. 95:90149018

van der Lek, H. A. A. 1919. Over de z.g. "verwelkingszoekten," in het bijzonder welke door Verticillium alboatrum veroorzaakt worden. Tijdschr. Plantenziekten. 25:17-52

Li, H. 2013. Aligning sequence reads, clone sequences and assembly contigs with BWA-MEM. arXiv. 0०:1-3

Li, H., Handsaker, B., Wysoker, A., Fennell, T., Ruan, J., Homer, N., Marth, G., Abecasis, G., and Durbin, R. 2009. The Sequence Alignment/ Map format and SAMtools. Bioinformatics. 25:2078-2079
Li, H., Hearne, S., Bänziger, M., Li, Z., and Wang, J. 2010. Statistical properties of QTL linkage mapping in biparental genetic populations. Heredity (Edinb). 105:257-267

Li, J. 2019. Identification of host-specific effectors mediating pathogenicity of the vascular wilt pathogen.

Liedl, B. e., Labate, J. A., Stommel, J. R., Slade, A., and Kole, C. 2013. Mapping mendelian factors underlying quantitative traits using RFLP linkage maps. CRC Press. ISBN 9781457710957

Lievens, B., Brouwer, M., Vanachter, A. C. R. C., Cammue, B. P. A., and Thomma, B. P. H. J. 2006. Real-time PCR for detection and quantification of fungal and oomycete tomato pathogens in plant and soil samples. Plant Sci. 171:155-165

Livak, K. J., and Schmittgen, T. D. 2001. Analysis of relative gene expression data using real-time quantitative PCR and the 2(-Delta Delta C(T)) Method. Methods. 25:402-408

de los Reyes, B. G. 2019. Genomic and epigenomic bases of transgressive segregation - New breeding paradigm for novel plant phenotypes. Plant Sci. 288:110213

Lynch, D. R., Kawchuk, L. M., Hachey, J., Bains, P. S., and Howard, R. J. 1997. Identification of a gene conferring high levels of resistance against Verticillium wilt in Solanum chacoense. Plant Dis. 81:1011-1014

McMullen, M. D., Kresovich, S., Villeda, H. S., Bradbury, P., Li, H., Sun, Q., Flint-Garcia, S., Thornsberry, J., Acharya, C., Bottoms, C., Brown, P., Browne, C., Eller, M., Guill, K., Harjes, C., Kroon, D., Lepak, N., Mitchell, S. E., Peterson, B., Pressoir, G., Romero, S., Rosas, M. O., Salvo, S., Yates, H., Hanson, M., Jones, E., Smith, S., Glaubitz, J. C., Goodman, M., Ware, D., Holland, J. B., and Buckler, E. S. 2009. Genetic properties of the maize nested association mapping population. Science 325:737-740

Melotto, M., Underwood, W., Koczan, J., Nomura, K., and He, S. Y. 2006. Plant stomata function in innate immunity against bacterial invasion. Cell. 126:969-980

Mereschkowsky, C. 1905. Über natur und ursprung der chromatophoren im pflanzenreiche. Biol. Cent. 18:593-604 
Mesarich, C. H., Griffiths, S. A., Van Der Burgt, A., Ökmen, B., Beenen, H. G., Etalo, D. W., Joosten, M. H. A. J., and De Wit, P. J. G. M. 2014. Transcriptome sequencing uncovers the Avr5 avirulence gene of the tomato leaf mold pathogen Cladosporium fulvum. Mol. Plant-Microbe Interact. 27:846-857

Miah, G., Rafii, M. Y., Ismail, M. R., Puteh, A. B., Rahim, H. A., Asfaliza, R., and Latif, M. A. 2013. Blast resistance in rice: A review of conventional breeding to molecular approaches. Mol. Biol. Rep. 40:2369-2388

Michaelson, J. J., Loguercio, S., and Beyer, A. 2009. Detection and interpretation of expression quantitative trait loci (eQTL). Methods. 48:265276

Mikaberidze, A., and McDonald, B. A. 2020. A tradeoff between tolerance and resistance against a major fungal pathogen in elite wheat cultivars. New Phytol. :879-890

Network, R. P. 2016. Trends and challenges in pesticide resistance detection. Trends Plant Sci. 21:834-853

Niks, R. E., Qi, X., and Marcel, T. C. 2015. Quantitative resistance against biotrophic filamentous plant pathogens: concepts, misconceptions, and mechanisms. Annu. Rev. Phytopathol. 53:150605182533006

Oerke, E.-C. 1999. Estimated crop losses due to pathogens, animal pests and weeds. p. 72-741 Elsevier Science B.V.

Oerke, E. C. 2006. Crop losses to pests. J. Agric. Sci. 144:31-43

Okie, W. R., and Gardner, R. G. 1982a. Breeding for resistance against Verticillium dahliae race 2 of tomato in North Carolina. J. Am. Soc. Hortic. Sci. 107:552-555

Okie, W. R., and Gardner, R. G. 1982b. Screening tomato seedlings for resistance against Verticillium dahliae races 1 and 2. Plant Dis. 66:34-37

Ownley, B. H., and Trigiano, R. N. 2017. Plant Pathology: Concepts and laboratory exercises. CRC Press.

Pagán, I., Alonso-Blanco, C., and García-Arenal, F. 2008. Host responses in life-history traits and tolerance to virus infection in Arabidopsis thaliana. PLoS Pathog. 4

Pagán, I., and García-Arenal, F. 2018. Tolerance to plant pathogens: Theory and experimental evidence. Int. J. Mol. Sci. 19
Pak, T. R., Lynch, G. R., and Tsai, P. S. 1997. Modification of a CTAB DNA extraction protocol for plants containing high polysaccharide and polyphenol components sue. Plant Mol. Biol. Report. 15:8-15

Pandolfi, V., Neto, J., Silva, M., Amorim, L., Wanderley-Nogueira, A., Silva, R., Kido, E., Crovella, S., and Iseppon, A. 2016. Resistance (r) genes: applications and prospects for plant biotechnology and breeding. Curr. Protein Pept. Sci. 18:323-334

Papadaki, A. M., Bletsos, F. A., Eleftherohorinos, I. G., Menexes, G., and Lagopodi, A. L. 2017. Effectiveness of seven commercial rootstocks against Verticillium wilt and their effects on growth, yield, and fruit quality of tomato. Crop Prot. 102:25-31

Parisi, M., Aversano, R., Graziani, G., Ruggieri, V., Senape, V., Sigillo, L., and Barone, A. 2016. Phenotypic and molecular diversity in a collection of 'Pomodoro di Sorrento' Italian tomato landrace. Sci. Hortic. (Amsterdam). 203:143-151

Parlevliet, J. E. 2002. Durability of resistance against fungal, bacterial and viral pathogens; present situation. Euphytica. 124:147-156

Pavan, S., Jacobsen, E., Visser, R. G. F., and Bai, Y. 2010. Loss of susceptibility as a novel breeding strategy for durable and broad-spectrum resistance. Mol. Breed. 25:1-12

Pegg, G. F., and Brady, B. L. 2002 . Verticillium wilts. $\mathrm{CAB}$ International, CABI Publishing.

Pegg, G. F., and Dixon, G. R. 1969. The reactions of susceptible and resistant tomato cultivars to strains of Verticillium albo-atrum. Ann. Appl. Biol. 63:389-400

Petersen, T. N., Brunak, S., Von Heijne, G., and Nielsen, H. 2011. SignalP 4.0: Discriminating signal peptides from transmembrane regions. Nat. Methods. 8:785-786

Pilet-Nayel, M. L., Moury, B., Caffier, V., Montarry, J., Kerlan, M. C., Fournet, S., Durel, C. E., and Delourme, R. 2017. Quantitative resistance against plant pathogens in pyramiding strategies for durable crop protection. Front. Plant Sci. 8:1-9

Poland, J. A., Balint-Kurti, P. J., Wisser, R. J., Pratt, R. C., and Nelson, R. J. 2009. Shades of gray: the world of quantitative disease resistance. Trends Plant Sci. 14:21-29 
Politowski, K. 1978. Tolerance and resistance against plant disease: an epidemiological study. Phytopathology. 68:1177

Preedy, V. R., and Watson, R. R. 2019. Tomatoes and tomato products: nutritional, medicinal and therapeutic properties. CRC Press. ISBN 9780367452759

Quinlan, A. R., and Hall, I. M. 2010. BEDTools: A flexible suite of utilities for comparing genomic features. Bioinformatics. 26:841-842

Råberg, L. 2014. How to live with the enemy: understanding tolerance to parasites. PLoS Biol. 12

Raboin, L. M., Ballini, E., Tharreau, D., Ramanantsoanirina, A., Frouin, J., Courtois, B., and Ahmadi, N. 2016. Association mapping of resistance against rice blast in upland field conditions. Rice. 9:1-12

Rick, C. M., and Chetelat, R. T. 1995. Utilization of related wild species for tomato improvement. Acta Hortic. :21-38

Rick, C. M., Martin, F. M., and Gentile, A. 1959. Linkage of Verticillium resistance $(\mathrm{Ve})$. Tomato Genet. Coop. 9:44

Robert, X., and Gouet, P. 2014. Deciphering key features in protein structures with the new ENDscript server. Nucleic Acids Res. 42:320324

Roberts, J. J. 1984. Tolerance to leaf rust in susceptible wheat cultivars. Phytopathology. 74:349

Robinson, D. B. 1957. Verticillium wilt of potato in relation to symptoms, epidemiology and variability of the pathogen. Pages 49 in: University of Wisconsin Agricultural Experiment Station Results Bulletin,

Robinson, J. T., Thorvaldsdóttir, H., Winckler, W., Guttman, M., Lander, E. S., Getz, G., and Mesirov, J. P. 2011. Integrative Genome Viewer. Nat. Biotechnol. 29:24-26

Rose, L. E., Overdijk, E. J. R., and van Damme, M. 2019. Small RNA molecules and their role in plant disease. Eur. J. Plant Pathol. 18:1-14

Rovenich, H., Boshoven, J. C., and Thomma, B. P. H. J. 2014. Filamentous pathogen effector functions: Of pathogens, hosts and microbiomes. Curr. Opin. Plant Biol. 20:96-103

Roy, B. A., and Kirchner, J. W. 2000. Evolutionary dynamics of pathogen resistance and tolerance. Evolution (N. Y). 54:51-63
Rudolph, B. A. 1931. Verticillium hadromicosis. Hilgardia. 5:201-900

Rygulla, W., Snowdon, R. J., Friedt, W., Happstadius, I., Cheung, W.Y., and Chen, D. 2008. Identification of quantitative trait loci for resistance against Verticillium longisporum in oilseed rape (Brassica napus). Phytopathology. 98:215-221

de Sain, M., and Rep, M. 2015. The role of pathogensecreted proteins in fungal vascular wilt diseases. Int. J. Mol. Sci. 16:23970-23993

Salvaudon, L., and Shykoff, J. A. 2013. Variation in Arabidopsis developmental responses to oomycete infection: resilience vs changes in life history traits. New Phytol. 197:919-926

Santhanam, P., Van Esse, H. P., Albert, I., Faino, L., Nürnberger, T., and Thomma, B. P. H. J. 2013 Evidence for functional diversification within a fungal Nepi-like protein family. Mol. PlantMicrobe Interact. 26:278-286

Schaible, L., Cannon, O. S., and Waddoups, V. 1951. Inheritance of resistance against Verticillium wilt in a tomato cross. Phytopathology. 41:986-990

van Schie, C. C. N. N., and Takken, F. L. W. W. 2014. Susceptibility genes 101: how to be a good host. Annu. Rev. Phytopathol. 52:1-31

Schneider, C. A., Rasband, W. S., and Eliceiri, K. W. 2012. NIH Image to ImageJ: 25 years of image analysis. Nat. Methods. 9:671-675

Schouten, H. J., Tikunov, Y., Verkerke, W., Finkers, R., Bovy, A., Bai, Y., and Visser, R. G. F. 2019. Breeding has increased the diversity of cultivated tomato in the Netherlands. Front. Plant Sci. 10:1-12

Shan, W., Cao, M., Leung, D., and Tyler, B. M. 2004. The Avrib locus of Phytophthora sojae encodes an elicitor and a regulator required for avirulence on soybean plants carrying resistance gene Rpsib. Mol. PlantMicrobe Interact. 17:394-403

Sherbakoff, C. D. 1949. Breeding for resistance against Fusarium and Verticillium wilts. Bot. Rev. 15:377-422

Shittu, H. O., Castroverde, D. C. M., Nazar, R. N., and Robb, J. 2009. Plant-endophyte interplay protects tomato against a virulent Verticillium. Planta. 229:415-426

Shivaprasad, P. V., Dunn, R. M., Santos, B. A. C. M., Bassett, A., and Baulcombe, D. C. 2012. Extraordinary transgressive phenotypes of hybrid tomato are influenced by epigenetics and small silencing RNAs. EMBO J. 31:257-266 
Short, D. P. G., Gurung, S., Hu, X., Inderbitzin, P., and Subbarao, K. V. 2014. Maintenance of sexrelated genes and the co-Occurrence of both mating types in Verticillium dahliae. PLoS One. 9

Sim, S. C., Durstewitz, G., Plieske, J., Wieseke, R., Ganal, M. W., van Deynze, A., Hamilton, J. P., Buell, C. R., Causse, M., Wijeratne, S., and Francis, D. M. 2012. Development of a large SNP genotyping array and generation of highdensity genetic maps in tomato. PLoS One. 7

Solanaceae Coordinated Agricultural Project. 2019. http://solcap.msu.edu/tomato_genotype_data. shtml.

Song, J., Bradeen, J. M., Naess, S. K., Raasch, J. A., Wielgus, S. M., Haberlach, G. T., Liu, J., Kuang, H., Austin-Phillips, S., Buell, C. R., Helgeson, J. P., and Jiang, J. 2003. Gene RB cloned from Solanum bulbocastanum confers broad spectrum resistance against potato late blight. Proc. Natl. Acad. Sci. U. S. A. 100:9128-9133

Song, Y., Zhang, Z., Seidl, M. F., Majer, A., Jakse, J., Javornik, B., and Thomma, B. P. 2016. Broad taxonomic characterization of Verticillium wilt resistance genes reveals ancient origin of the tomato Vei immune receptor. Mol. Plant Pathol. 18: 195-209

Spooner, D. M., Anderson, G. J., and Jansen, R. K. 1993. Chloroplast DNA Evidence for the Interrelationships of Tomtoes, Potatoes, and Pepinos (Solanaceae). Am. J. Bot. 80:676

St.Clair, D. A. 2010. Quantitative disease resistance and quantitative resistance loci in breeding. Annu. Rev. Phytopathol. 48:247-268

Stamatakis, A. 2014. RAxML version 8: A tool for phylogenetic analysis and post-analysis of large phylogenies. Bioinformatics. 30:1312-1313

Stamova, L. 2006. Introgression of resistance against Verticillium dahliae race 2 into processing tomato cultivars in california. Acta Hortic. 724:39-43

Stamova, L. 2005. Resistance against Verticillium dahliae race 2 and its introgression into processing tomato cultivars. Acta Hortic. 695:257-261

Stanke, M., Tzvetkova, A., and Morgenstern, B. 2006. AUGUSTUS at EGASP: using EST, protein and genomic alignments for improved gene prediction in the human genome. Genome Biol. $7: 1-8$
Stare, T., Ramšak, Ž., Blejec, A., Stare, K., Turnšek, N., Weckwerth, W., Wienkoop, S., Vodnik, D., and Gruden, K. 2015. Bimodal dynamics of primary metabolism-related responses in tolerant potato-Potato virus $\mathrm{Y}$ interaction. BMC Genomics. 16:1-17

Staskawicz, B. J., Dahlbeck, D., and Keen, N. T. 1984. Cloned avirulence gene of Pseudomonas syringae pv. glycinea determines race-specific incompatibility on Glycine max (L.) Merr. Proc. Natl. Acad. Sci. U. S. A. $81: 6024-6028$

Stergiopoulos, I., De Kock, M. J. D., Lindhout, P., and De Wit, P. J. G. M. 2007. Allelic variation in the effector genes of the tomato pathogen Cladosporium fulvum reveals different modes of adaptive evolution. Mol. Plant-Microbe Interact. 20:1271-1283

Stukenbrock, E. H., and McDonald, B. A. 2008. The origins of plant pathogens in agro-ecosystems. Annu. Rev. Phytopathol. 46:75-100

Tabaeizadeh, Z., Agharbaoui, Z., Harrak, H., and Poysa, V. 1999. Transgenic tomato plants expressing a Lycopersicon chilense chitinase gene demonstrate improved resistance against Verticillium dahliae race 2. Plant Cell Rep. 19:197-202

Taguchi, F., Takeuchi, K., Katoh, E., Murata, K., Suzuki, T., Marutani, M., Kawasaki, T., Eguchi, M., Katoh, S., Kaku, H., Yasuda, C., Inagaki, Y., Toyoda, K., and Shiraishi, T. 2006. Identification of glycosylation genes and glycosylated amino acids of flagellin in Pseudomonas syringae pv. tabaci. 8:923-938

Taguchi, F., Yamamoto, M., Ohnishi-kameyama, M., Iwaki, M., Yoshida, M., Ishii, T., and Konishi, T. 2010. Defects in flagellin glycosylation affect the virulence of Pseudomonas syringae pv . tabaci 6605. :72-80

Takken, F. L. W., Luderer, R., Gabriëls, S. H. E. J., Westerink, N., Lu, R., De Wit, P. J. G. M., and Joosten, M. H. A. J. 2000. A functional cloning strategy, based on a binary PVX-expression vector, to isolate HR-inducing cDNAs of plant pathogens. Plant J. 24:275-283

Takken, F. L. W., Thomas, C. M., Joosten, M. H. A. J., Golstein, C., Westerink, N., Hille, J., Nijkamp, H. J. J., De Wit, P. J. G. M., and Jones, J. D. G. 1999. A second gene at the tomato Cf-4 locus confers resistance against Cladosporium fulvum through recognition of a novel avirulence determinant. Plant J. 20:279-288 
Talboys, P. W. 1972. Resistance against vascular wilt fungi. Proc. R. Soc. London. 181:319-332

Tang, J., and Chu, C. 2017. MicroRNAs in crop improvement: Fine-tuners for complex traits. Nat. Plants. 3

Taylor, J., and Butler, D. 2017. R-Package ASMap: efficient genetic linkage map construction and diagnosis. 79

Thomma, B. P. H. J., Nürnberger, T., and Joosten, M. H. A. J. 2011. Of PAMPs and effectors: the blurred PTI-ETI dichotomy. Plant Cell. 23:4-15

Tjamos, E. C. 1976. Virulence of Greek Verticillium isolates on monogenically resistant and susceptible tomato seedlings. Pages 41 in: 2 nd International Verticillium Symposium,

Toppino, L., and Barchi, L. 2016. QTLs mapping for Fusarium oxysporum and Verticillium dahliae resistance in eggplant (Solanum melongena L. Pages 248-255 in: Proceedings of XVIth EUCARPIA Capsicum and Eggplant Working Group Meeting,

Tsolakidou, M. D., Pantelides, I., Tzima, A. K., Kang, S., Paplomatas, E. J., and Tsaltas, D. 2019. Disruption and overexpression of the gene encoding ACC (1-aminocyclopropane-1carboxylic acid) deaminase in soil-borne fungal pathogen Verticillium dahliae revealed the role of ACC as a potential regulator of virulence and plant defence. Mol. Plant-Microbe Interact. 32:639-653

Tuchinda, C., Srivannaboon, S., and Lim, H. W. 2006. Photoprotection by window glass, automobile glass, and sunglasses. J. Am. Acad. Dermatol. 54:845-854

Tuinstra, M. R., Ejeta, G., and Goldsbrough, P. B. 1997. Heterogeneous inbred family (HIF) analysis: A method for developing near-isogenic lines that differ at quantitative trait loci. Theor. Appl. Genet. 95:1005-1011

Uribe, P., Jansky, S., and Halterman, D. 2014. Two CAPS markers predict Verticillium wilt resistance in wild Solanum species. Mol. Breed. 33:465-476

Usami, T., Momma, N., Kikuchi, S., Watanabe, H., Hayashi, A., Mizukawa, M., Yoshino, K., and Ohmori, Y. 2017. Race 2 of Verticillium dahliae infecting tomato in Japan can be split into two races with differential pathogenicity on resistant rootstocks. Plant Pathol. 66:230-238
Use, T. H. E., Wild, O. F., Species, L., and Tomato, F. O. R. 1953. The use of wild Lycopersicon species for tomato disease control. Phytopathology. 44:409-414

Vales, M. I., Schön, C. C., Capettini, F., Chen, X. M., Corey, A. E., Mather, D. E., Mundt, C. C., Richardson, K. L., Sandoval-Islas, J. S., Utz, H. F., and Hayes, P. M. 2005. Effect of population size on the estimation of QTL: A test using resistance against barley stripe rust. Theor. Appl. Genet. 111:1260-1270

Veronese, P., Narasimhan, M. L., Stevenson, R. A., Zhu, J. K., Weller, S. C., Subbarao, K. V., and Bressan, R. A. 2003 . Identification of a locus controlling Verticillium disease symptom response in Arabidopsis thaliana. Plant J. 35:574-587

Víquez-Zamora, M., Myluska, C., Finkers, R., Tikunov, Y., Bovy, A., Visser, R. G., Bai, Y., and van Heusden, S. 2014. Mapping in the era of sequencing: high density genotyping and its application for mapping TYLCV resistance in Solanum pimpinellifolium. BMC Genomics. 15

Vleeshouwers, V. G. A. A., and Oliver, R. P. 2014. Effectors as tools in disease resistance breeding against biotrophic, hemibiotrophic, and necrotrophic plant pathogens. Mol. PlantMicrobe Interact. 27:196-206

Vleeshouwers, V. G. A. A, Raffaele, S., Vossen, J. H., Champouret, N., Oliva, R., Segretin, M. E., Rietman, H., Cano, L. M., Lokossou, A., Kessel, G., Pel, M. A, and Kamoun, S. 2011. Understanding and exploiting late blight resistance in the age of effectors. Annu. Rev. Phytopathol. 49:507-31

Van Der Vossen, E., Sikkema, A., Te Lintel Hekkert, B., Gros, J., Stevens, P., Muskens, M., Wouters, D., Pereira, A., Stiekema, W., and Allefs, S. 2003. An ancient $R$ gene from the wild potato species Solanum bulbocastanum confers broadspectrum resistance against Phytophthora infestans in cultivated potato and tomato. Plant J. 36:867-882

Wang, H. M., Lin, Z. X., Zhang, X. L., Chen, W., Guo, X. P., Nie, Y. C., and Li, Y. H. 2008. Mapping and quantitative trait loci analysis of Verticillium wilt resistance genes in cotton. J. Integr. Plant Biol. 50:174-182 
Wang, M., Weiberg, A., Lin, F. M., Thomma, B. P. H. J., Huang, H. Da, and Jin, H. 2016. Bidirectional cross-kingdom RNAi and fungal uptake of external RNAs confer plant protection. Nat. Plants. 2

Weiberg, A., Bellinger, M., and Jin, H. 2015. Conversations between kingdoms: small RNAs. Curr. Opin. Biotechnol. 32:207-215

Wick, R. 2018. Porechop.

Wikström, N., Savolainen, V., and Chase, M. W. 2001. Evolution of the angiosperms: Calibrating the family tree. Proc. R. Soc. B Biol. Sci. 268:2211-2220

Wilhelm, S. J. 1955. Longevity of the Verticillium wilt fungus in the laboratory and field. Phytopathology. 45:180-181

Win, J., Chaparro-Garcia, A., Belhaj, K., Saunders, D. G. O., Yoshida, K., Dong, S., Schornack, S., Zipfel, C., Robatzek, S., Hogenhout, S. A., and Kamoun, S. 2012. Effector biology of plant-associated organisms: Concepts and perspectives. Cold Spring Harb. Symp. Quant. Biol. 77:235-247

Windsor, D. A. 1998. Most of the species on Earth are parasites. Int. J. Parasitol. 28:1939-1941

Yadeta, K. A. 2012. PhD Thesis, Wageningen University. Verticillium wilt resistance in Arabidopsis and tomato: identification and functional characterization.

Yadeta, K. A., Thomma, B. P. H. J., and J Thomma, B. P. H. 2013. The xylem as battleground for plant hosts and vascular wilt pathogens. Front. Plant Sci. 4:97

Yang, L., Mu, X., Liu, C., Cai, J., Shi, K., Zhu, W., and Yang, Q. 2015. Overexpression of potato miR482e enhanced plant sensitivity to Verticillium dahliae infection. J. Integr. Plant Biol. 57:1078-1088

Yu, L.-X., Zhang, F., Culma, C. M., Lin, S., Niu, Y., Zhang, T., Yang, Q., Smith, M., and Hu, J. 2020. Construction of high-density linkage maps and identification of quantitative trait loci associated with Verticillium wilt resistance in autotetraploid alfalfa (Medicago sativa l.). Plant Dis. 104:1439-1444

Zalom, F. G. 2003. Pests, endangered pesticides and processing tomatoes. Acta Hortic. 613:223-233
Zamir, D., Bolkan, H., Juvik, J. A., Watterson, J. C., and Tanksley, S. D. 1993. New evidence for placement of $\mathrm{Ve}$ - the gene for resistance against Verticillium race 1. Tomato Genet. Coop. 43:51-52

Zeise, K., and Von Tiedemann, A. 2002. Host specialization among vegetative compatibility groups of Verticillium dahliae in relation to Verticillium longisporum. J. Phytopathol. 150:112-119

Zhang, J., Yu, J., Pei, W., Li, X., Said, J., Song, M., and Sanogo, S. 2015. Genetic analysis of Verticillium wilt resistance in a backcross inbred line population and a meta-analysis of quantitative trait loci for disease resistance in cotton. BMC Genomics. 16:1-13

Zhang, L., Peng, Y., Wei, X., Dai, Y., Yuan, D., Lu, Y., Pan, Y., and Zhu, Z. 2014a. Small RNAs as important regulators for the hybrid vigour of super-hybrid rice. J. Exp. Bot. 65:5989-6002

Zhang, X., Yuan, Y., Wei, Z., Guo, X., Guo, Y., Zhang, S., Zhao, J., Zhang, G., Song, X., and Sun, X. 2014b. Molecular mapping and validation of a major QTL conferring resistance against a defoliating isolate of verticillium wilt in cotton (Gossypium hirsutum L.). PLoS One. 9:11-17

Zhang, Z., Song, Y., Liu, C. M., and Thomma, B. P. H. J. 2014c. Mutational analysis of the Ver immune receptor that mediates Verticillium resistance in tomato. PLoS One. 9:1-10

Zhao, Y., Wang, H., Chen, W., and Li, Y. 2014. Genetic structure, linkage disequilibrium and association mapping of Verticillium wilt resistance in elite cotton (Gossypium hirsutum L.) germplasm population. PLoS One. 9 



\section{Summary}

\section{Acknowledgements}

About the author

\section{Education statement}





\section{Summary}

Verticillium dahliae is a soil-borne fungal pathogen that causes vascular wilt diseases in temperate regions around the world. The fungus can infect over 200 plant species, among which are many economically important crops. Yield losses caused by $V$. dahliae generally range from 10 to $15 \%$, but for various crops, such as potato, cotton, lettuce and strawberry, yield losses of over $50 \%$ have been reported. Because the fungus resides inside the plant for the majority of its life cycle, most fungicides are ineffective. For over a century, the identification and employment of genetic resistance has been one of the preferred strategies to control $V$. dahliae. In tomato, the only described qualitative resistances thus far against $V$. dahliae are the Ver gene and the dominantly inherited $V 2$ locus. Soon after the introduction in commercial tomato cultivars of Ve1 in the 1950 and V2 in 2006, race 2 and 3 strains emerged that have overcome the resistance mediated by Ve1 and V2, respectively. Thus, identification and employment of additional resistances against $V$. dahliae is required to further control $V$. dahliae, which is the aim of this $\mathrm{PhD}$ thesis.

In order to reliably explore germplasm for resistance against $V$. dahliae, a precise phenotyping method is very important. The phenotyping method should provide a strong discriminative power, such that quantitative differences in $V$. dahliae symptom expression between host genotypes can effectively be detected. Therefore, in Chapter 2, we compared and validated multiple phenotyping methods. We demonstrated that of the methods we tested, comparing the canopy area of $V$. dahliae-inoculated plants with that of mockinoculated plants yields the best discriminative power. Furthermore, we found that the discriminative power to detect differences in symptom expression between genotypes was higher when the canopy area was measured at 21 days post inoculation than at 14 days post inoculation. In the remainder of the $\mathrm{PhD}$ research, we therefore used the optimized phenotyping approach for $V$. dahliae experiments on tomato. In an attempt to further optimize the assay, we also evaluated several inoculation methods. Neither increasing the $V$. dahliae inoculum concentration, nor trimming of the roots prior to inoculation, nor nutrient supplementation increased the discriminative power of the assay.

In Chapter 3, we compared $V$. dahliae symptom development on six wild tomato accessions that were previously reported to possess a particular degree of resistance to $V$. dahliae. We finally focused on three wild accessions, Solanum cheesmanii VG-20, Solanum pimpinellifolium VG-21, and Solanum pimpinellifolium VG-3. Of these accessions, especially VG-20 and VG21 displayed a strong reduction in $V$. dahliae symptoms. We then subjected these three accessions to a diverse collection of $V$. dahliae strains and a $V$. nonalfalfa strain. The three accessions displayed reduced symptom expression to nearly all strains tested. By measuring the colonization of several of these strains in the lower stems of the plants, we confirmed that especially VG-20 and VG-21 displayed a broad-spectrum resistance against V. dahliae strains. 
To unravel the genetics of the resistance of VG-20, VG-21 and VG-3, F6 recombinant inbred line (RIL) populations were developed with $S$. lycopersicum Moneymaker as the susceptible parent. In Chapter 4, we screened the resistance of the RIL populations of VG-3 and VG-21. In both populations, the stunting symptoms displayed a continuous segregation pattern, indicating that VG-3 and VG-21 possess quantitative resistance against $V$. dahliae that is probably based on multiple genes. Unfortunately, we were unable to identify QTLs based on the stunting symptoms, neither in the VG-21 population, nor in the VG-3 population. Possibly, the low estimated heritability and reproducibility of the symptoms, combined with the likely polygenic nature of the resistance hampered the detection of QTLs. However, the VG-3 population also segregated for yellowing symptoms on the lower leaves. Since yellowing symptoms were not apparent in most mock-inoculated plants and no meaningful correlation emerged between the yellowing of leaves of mock-inoculated plants and $V$. dahliae-inoculated plants, we concluded that this yellowing is most likely caused by the $V$. dahliae infection. Strikingly, the segregation pattern of the yellowing symptoms was vastly different from the segregation pattern of the stunting symptoms, suggesting a different genetic basis for both symptoms. Both the V. dahliae-inoculated F1 and many of the RILs developed more yellowing leaves than both parents, which suggests that heterozygosity and transgressive segregation caused an increase in yellowing symptoms on $V$. dahliaeinoculated plants. Based on the yellowing symptom, two QTLs from the susceptible parent lycopersicum Moneymaker could be identified, yet no QTLs from VG-3.

In Chapter 5, we describe the QTL analysis for the resistance of VG-20. Similar to what we observed in the VG-21 and VG-3 RIL populations, we observed a continuous distribution of stunting levels in the VG-20 RIL population. This suggests that also VG-20 possesses quantitative resistance against $V$. dahliae that is probably based on multiple genes. Fortunately, in contrast to the previous chapter, we identified five QTLs associated with the stunting symptom. Three QTLs originated from VG-20, whereas the two other QTLs originated from Moneymaker. Although RILs homozygous for the VG-20 allele of the three QTLs from VG-20 all displayed reduced stunting compared to RILs with the Moneymaker allele, they still displayed a higher level of stunting than VG-20 itself, suggesting that these QTLs may not fully account for the resistance of VG-20. Interestingly, one of the QTLs contributed by Moneymaker on chromosome 3 overlapped with one of the QTLS we identified in the VG-3 population. However, as the QTL in the VG-3 population was associated with reduced yellowing symptoms and the QTL in the VG-20 population with reduced stunting symptoms, it is not clear if these are truly the same QTL. Still, the notion that we identified QTLs originating from Moneymaker in both populations suggests that despite being universally used as a susceptible control, Moneymaker still possesses some degree of basal defence against $V$. dahliae. 
In Chapter 6, we performed a comparative genomics analysis between race 2 and race 3 strains of $V$. dahliae in an attempt to identify the gene that encodes the enigmatic "Av2" avirulence effector, which activates the resistance governed by $V 2$. In order to categorize $V$. dahliae strains into race 2 and 3, we performed pathogenicity assays on V2-resistant plants. Using nanopore sequencing, the race 2 and 3 strains were sequenced. Our comparative genomics analysis identified $277 \mathrm{~kb}$ of race 2 -specific sequences, which are absent from race 3 strains. Ultimately, in these race 2 -specific sequences, we identified two effector candidates which were expressed in planta and of which the protein products were predicted to be secreted. Likely, one these effector candidates is the avirulence molecule Av2 that activates $V 2$ resistance. Importantly, since both effector candidates were absent from V. dahliae strains that originated from the USA, Canada, China and the Netherlands, this suggests that race 3 strains are already present around the world, and that additional resistances besides $V e 1$ and $V 2$ may be required to control $V$. dahliae in these regions.

Finally, in Chapter 7, the most important results presented in this $\mathrm{PhD}$ thesis are integrated and discussed, to provide a perspective how these findings may be used to control $V$. dahliae in tomato and other crops. 



\section{Acknowledgements}

After an amazing, inspiring, and intense adventure, the time has finally come that my $\mathrm{PhD}$ thesis is finished. Although my $\mathrm{PhD}$ has had its ups and downs, the ups definitively outweighed the downs. I never regretted embarking on this amazing journey, and I have learned a lot, both on a personal and professional level. Looking back, it goes without saying that this $\mathrm{PhD}$ work is the result of a concerted effort. I could not have come where I am today without the many people who helped and supported me along the way.

First, I would like to thank my supervisors Bart, Henk and Yuling. I am very grateful that you gave me the opportunity me to start with this project, which connected perfectly to my interests and previous experience. Furthermore, I have always considered it a privilege to have been part of two different laboratories. Although it can sometimes be a bit challenging to have three supervisors with different personalities, backgrounds and viewpoints, the diversity of your support, feedback and our discussions has definitively enriched my $\mathrm{PhD}$ research.

Henk, thank you for being my mentor, and for your supervision and dedication throughout the whole $\mathrm{PhD}$ project. In fact, even before the PhD project started, you already invited me to have a look when the first crosses were made which would be the start of the populations described in this thesis. You made sure that your office was always open for me, and I would like to thank you for all the nice discussions we've had throughout the years, from puzzling with the genetics of the "AAs" and "BBs", to the "Verticillium challenges" we faced, to your sailing adventures. I also really enjoyed the dinners with the "Schoutens" group in your home, so also thanks for opening your home.

Yuling, ever since I started my MSc in Wageningen, you have inspired and guided me, first as a teacher and study advisor and then as my promotor. I still remember the discussions we had when you were my study advisor and I could not decide whether I wanted to become a breeder of phytopathologist. I guess in a way, by giving me the opportunity to do this $\mathrm{PhD}$, you made sure I never really had to make that choice. Although we can both be a bit stubborn at times, I always greatly valued your contributions and suggestions, especially your unique plant breeders' point of view, which will definitively help me in the rest of my career.

Bart, since our very first chat I directly felt welcome at the laboratory of phytopathology and in the Verticillium group. You have a special combination of being a passionate, dedicated, and serious researcher who at the same time is also an honest but caring mentor and supervisor. I have always felt privileged to have had you, besides two plant breeding experts, as one of my promotors. Your feedback and suggestions definitively made me better appreciate and understand the more fundamental aspects of phytopathology. I wish you all the best in Cologne!

Ton, thanks for being my external advisor. Although we did not need to meet very often, it was nice to be able to talk to you and to discuss the progress of my research. 
Besides having had the luxury of doing my $\mathrm{PhD}$ research in two laboratories, I really enjoyed the collaboration with the companies in the Verticillium project. Thank you, Bejo, EastWest, Hazera, Nunhems, Rijk Zwaan, Syngenta and Takii for your dedicated involvement in the project. I really valued your feedback during the half-yearly consortium meetings. Your input in the project definitively was of value for my $\mathrm{PhD}$ research.

Next, I would like to thank the dedicated staff of Unifarm. Especially Bert, Bertus, Andre, Sean, Henk, Teus and Maarten, thanks for your help and support with my countless greenhouse experiments. I did some calculations, and overall, you have filled more than $\underline{21 \text { thousand pots }}$ for me! It thus goes without saying that without your help, I would not have been where I am now.

Annelies, Bernadette, Ciska, Danny, Doret, Grady, Gerard, Gert, Iris, Irma, Isolde, Laurens, Linda, and Johan you made sure I could always perform my lab work in well-organized, wellfunctioning and vibrant labs, both at PBR and at Phyto. Thanks for always being available to help me or answer any of my questions. Furthermore, the past and present secretaries of both laboratories also deserve my gratitude for all their great work, so thank you Ali, Danielle, Janneke, Letty, Nicole and Petra.

Fien, thanks for your help with providing the tomato seeds for my experiments. You were always available when I had questions about plant passports, crossings or strange-looking wild tomato plants. You even taught me how to cross tomato plants, which is, of course, an important skill for any plant breeding $\mathrm{PhD}$.

Danny, thanks for always being one of the happiest faces in the lab. It is always fun talking to you, and I will miss our little chats in the corridor. Also, thanks for helping me out with my last experiments, as without you I would probably still have been grinding stems up to this day. Furthermore, thanks to you and Doret, I kept my writing motivation up, as you made it impossible for me to 'escape' to the lab or the greenhouse.

Johan, thanks for your tips and advice when I was setting up a digital phenotyping method to score the Verticillium symptoms on tomato plants. Your help with developing a method to detect $\mathrm{QR}$-codes in pictures has probably saved me weeks of tedious manual image analysis.

I would also like to thank all my PBR colleagues, especially Aina, Anne, Annick, Charlotte, Cynara, Henriette, Jarst, Jeroen, Johan Bucher, Johan Willemsen, Kim, Mas, Pauline and Peter for the nice coffee breaks, lunches, lab outings and activities we shared. Thanks to you, I looked forward to lunch every day. Not only to (again) fill my belly with spicy noodles, but especially to enjoy the funny and often crazy stories and jokes we shared during lunch. I already miss the bewildered looks on the faces of people walking by who picked up snippets of our conversations.

Many thanks, of course, also go out to my "PhD buddy" Katharina. We embarked on our Verticillium journey together, and you have always been there for me throughout this journey. It 
has been great to be able to share all the ups and downs of our $\mathrm{PhD}$ lives, and I think although, or perhaps becáuse we are quite different persons, we have learned a lot from each other. I am very proud of what we achieved together, and I'm happy to have shared this $\mathrm{PhD}$ journey together with you.

Kim, we also shared all the ups and downs of our $\mathrm{PhD}$ journey together from the very start. I will miss the coffee-breaks we had, all the walks around the little lake and the many cups of tea and coffee we enjoyed at Doppios. Also, I am really happy you wanted to be one of my paranymphs, and I am sure you will also successfully make it to the finish line.

Aina, thanks for being my fellow "QTL mapping buddy". I enjoyed our common quest to get familiar with the ins and outs of R/QTL, despite the often not-so-obvious instructions of Broman's manual. Also, thanks for the many cookies and pieces of fruit you shared with me during our coffee breaks, which I always forgot to bring with me.

Of course, I would also like to thank my colleagues and fellow PhDs at the laboratory of Phytopathology, for making me feel welcome from the very first day, even though I was mostly working at Plant Breeding. I really liked all the Christmas drinks, lab-outings, and sushidinners together. It was also always very helpful to present my "plant breeding" research to you, as many of your questions and suggestions made me think about my research in ways I otherwise wouldn't have.

Furthermore, I would like to thank the former and remaining members of the Verticillium group, especially David, Edgar, Gabriel, Grardy, Hui, Jasper, Jinling, Katharina, Martin, Malaika, Michael, Nick and Nelia, for the always warm and stimulating group environment, your commitment to the work discussions, and of course for enjoying the (very) spicy potluck dinners with some of you.

David, Edgar, Gabriel, Katharina and Sergio, thanks for all the nice dinners we have shared over the years. It was a great experience to get to know some of the South American cuisine, while enjoying (too) many glasses of wine together. I also really enjoyed our trips together to Germany and Belgium. Thanks for being such nice travel companions.

Finally, many thanks go out to my family, especially my amazing parents Walter and Joke and my wonderful sister Iris. Thanks for always being there for me, and for all your help and support. I really appreciate you always had time for one of our many hikes in the forest, which was a great way to clear my mind. Without you I would not have been where I am today. Iris, I am very happy that you are one of my paranymphs, and I am looking forward to taking the last hurdle of my $\mathrm{PhD}$ journey together with you.

Als laatste wil ik ook Opa Jan bedanken. Ook al bent $u$ niet meer onder ons, $u$ heeft mij altijd geïnspireerd om het beste uit mijzelf te halen. Ook bent $u$ de allereerste geweest die mij geïnspireerd heeft om later iets met planten te gaan doen, door altijd samen naar uw moestuin te gaan. Het gaat u goed! 



\section{About the author}

Jasper Pepijn Vermeulen was born in Utrecht on July $21^{\text {st }}, 1991$. He spent most of his youth in the Tulastraat in Voordorp, located in the suburbs of Utrecht. One of the very first words Jasper ever spoke was "buiten", meaning "outside" or "outdoors". Probably influenced by the many holiday trips Jasper undertook with his parents into the outdoors, Jasper has always been fascinated by the natural world. In 2010, he therefore started his BSc studies in biology at Utrecht University. Although he originally wanted to study ecology and evolutionary biology, the fascinating world of how plants adapt to biotic and abiotic stresses immediately caught his attention. He spent the

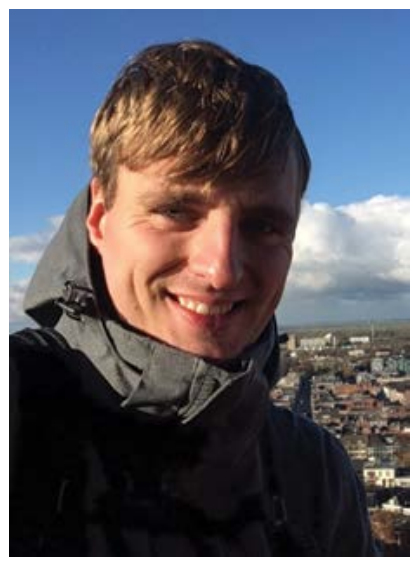
remainder of his BSc studies specializing in plant physiology and phytopathology. Jasper did his BSc thesis in the group of Prof. dr. R. (Ronald) Pierik, where he studied the effect of daylength on the shade avoidance response of Arabidopsis thaliana plants. After finishing his BSc thesis, Jasper took a little detour. He moved to the high Arctic for six months, where he joined the University Centre of Svalbard (UNIS) to study Arctic Biology. Although this was the greatest adventure of his life, his growing interest in phytopathology and plant breeding brought him back to civilization. He moved to Wageningen to do his MSc studies in plant biotechnology, where he specialized in molecular plant breeding and phytopathology. Jasper wrote two MSc theses at the laboratory of Plant Breeding: a minor thesis in the group of dr. JH (Jack) Vossen, and a major thesis in the group of dr.ir. RE (Rients) Niks. In his minor thesis, Jasper studied the role of helper NB-LRR proteins in the resistance of potato against Phythoptora infestans. In his major thesis, he worked on the identification of QTLs for the non-host resistance of barley against multiple formae speciales of powdery mildew. After completing his MSc studies, he continued as a PhD candidate in the group of prof. dr. Y (Yuling) Bai at the laboratory of Plant Breeding and the group of prof. dr. ir. BPHJ (Bart) Thomma at the laboratory of Phytopathology. In his $\mathrm{PhD}$ research, Jasper further combined his passion for plant breeding and phytopathology by searching for QTLs for the resistance of wild tomato plants against Verticillium dahliae. After he finished writing his PhD thesis, Jasper continued his journey as a phytopathology researcher at the Dutch seed company Rijk Zwaan.

Email: jasper.vermeulen@icloud.com 



\title{
Education Statement of the Graduate School Experimental Plant Sciences
}

\author{
Issued to: Jasper P. Vermeulen \\ Date: $\quad 20$ October 2020 \\ Group: $\quad$ Laboratory of Phytopathology, Laboratory of Plant Breeding \\ University: Wageningen University \& Research
}

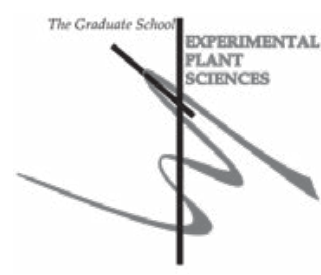

\section{1) Start-Up Phase}

date

$\underline{c p}$

- First presentation of your project

"Identification of genes for resistance or susceptibility to Verticillium wilt in

28 Jun 2016 tomato and other crops"

- Writing or rewriting a project proposal

- MSc courses

Subtotal Start-Up Phase

2) Scientific Exposure

$\underline{\text { date }}$

$\underline{c p}$

- EPS PhD student days

EPS Get2Gether 2017

EPS Get2Gether 2018

9 Feb 2017

0,3

EPS Get2Gether 2019

15-16 Feb 2018

0,6

EPS Get2Gether 2019

11-12 Feb $2019 \quad 0,6$

EPS Theme 2 Symposium 2017

23 Jan 2017

0,3

EPS Theme 2 Symposium 2018

24 Jan 2018

0,3

EPS Theme 2 Symposium 2019

1 Feb 2019

0,3

EPS Theme 2 Symposium 2020

4 Feb 2020

0,3

- Lunteren Days and other national platforms

Annual Experimental Plant Sciences Meeting in Lunteren

10-11 Apr $2017 \quad 0,6$

Annual Experimental Plant Sciences Meeting in Lunteren

9-10 Apr 2018

Annual Experimental Plant Sciences Meeting in Lunteren

8-9 Apr 2019

0,6

Host-Microbe Genetics meeting at the UMCG in Groningen

26 Oct 2018

0,6

TKI Verticillium consortium kick-off meeting May 2016

30 May 2016

0,3

TKI Verticillium consortium meeting November 2016

24 Nov 2016

0,2

TKI Verticillium consortium meeting May 2017

18 May 2017

0,2

TKI Verticillium consortium meeting November 2017

16 Nov 2017

0,2

TKI Networking Event 2018

3 Apr 2018

0,2

TKI Verticillium consortium meeting May 2018

31 May 2018

TKI Verticillium consortium meeting November 2018

5 Nov 2019

TKI Verticillium consortium meeting May 2019

16 May 2019

0,2

TKI Verticillium consortium meeting November 2019

14 Nov 2019

Seminars (series), workshops and symposia

Symposium: WGS WURomics symposium 2016

15 Dec 2016

Symposium: "Breeding Data: Statistical Advances in Modern Plant Breeding"

16 Oct 2018 
Seminar: Dr. Caitlin Allen - 'How Ralstonia solanacearum succeeds in plant xylem vessels'

Seminar: Wenbo Wa - 'Effectors as molecular probes to understand

29 Apr 2016

0,1

pathogenesis'

Seminar: Fons Debets

20 Jun $2016 \quad 0,1$

Seminar: Remco Stam

16 Dec 2016

0,1

Seminar: Asaf Levy - 'Bacteria and the future of agriculture: from sequence to

26 Jan 2018

0,1

22 Feb 2018

0,1

function'

Seminar: IBL Seminar Dr. Margaret Frank - 'Grafting-Induced Vigor in Crops:

19 Jun 2018

New Approaches to Understanding an Ancient Practice'

Seminar: Dr. Mary C. Wildermuth - 'Salicylic acid and cell cycle control of plantmicrobe interactions'

Seminar: Arjan de Visser

Seminar: Melvin Bolton - 'Sugarbeet pathology - from fungi to viruses'

25 Jun 2018

29 Jun 2018

Seminar: Volkel Lipka - 'Live and Let Die or Live and Let Live - Interactions of

7 Sep 2018

Arabidopsis with fungal pathogens'

14 Sep 2018

Seminar: Tom Wood - 'Nanopath: Utilising Nanopore sequencing for Septoria

14 Sep 2018 surveillance'

Seminar: Bjarne Larsen - 'Applying Evolutionary Theory to Improve Plant

24 Feb 2020

Production'

2 Apr 2020

Seminar: Nick Talbot - 'Investigating the biology of plant infection by rice blast fungus'

Seminar plus

- International symposia and congresses

EUCARPIA tomato 2018, Naples, Italy

2019 IS-MPMI XVIII Congress, Glasgow, Scotland

2-4 May $2018 \quad 0,9$

14-18 Jul $2019 \quad 1,5$

- Presentations

Oral presentation at TKI Verticillium consortium kick-off meeting May 2016

Oral presentation at TKI Verticillium consortium meeting November 2019

Poster Presentation at Annual Experimental Plant Sciences Meeting Lunteren,

30 May 2016

1,0

14 Nov $2019 \quad 1,0$

EUCARPIA Tomato 2018 and TKI Networking Event 2018

2018

Poster presentation at IS-MPMI XVIII Congress, Glasgow, Scotland

$15 \mathrm{Jul} 2019$

1,0

3rd year interview

- Excursions

Excursion to Tomato World

14 Oct 2016

0,2

Subtotal Scientific Exposure

\section{3) In-Depth Studies}

$\begin{array}{cc}\text { date } & \underline{c p} \\ & \\ \text { 18 May 2016 } & 0,3 \\ \text { 12-13 Dec 2016 } & 0,6 \\ \text { 29-31 Aug 2016 } & 1,4 \\ \text { 11-19 Dec 2017 } & 1,5 \\ \text { 5-6 Feb 2019 } & 0,6 \\ \text { 11-13 Jun 2019 } & 1,6\end{array}$


Journal club

Individual research training

Subtotal In-Depth Studies

6,0

4) Personal Development

$\begin{array}{cc}\text { date } & \underline{c p} \\ \text { 1 Sep 2016 } & 0,3 \\ \text { Apr - Jun 2017 } & 0,3 \\ \text { 2017/2018 } & 0,4 \\ \text { 7 Nov 2018 } & 0,3 \\ \text { 6 Mar 2019 } & 0,3 \\ \text { 12 Sep - 10 Oct 2019 } & 1,6 \\ \text { 24 Apr - 26 Jun 2019 } & 1,3 \\ \text { 4-5 Nov 2019 } & 0,6 \\ \text { 20 May 2017 } & 0,3 \\ \text { Aug 2017 - Dec 2018 } & 1,5 \\ \text { Sep 2018 - Jun 2019 } & 1,0 \\ & \end{array}$

- General skill training courses

Competence Assessment

WUR Meditation Course

Plant Breeding PhD Discussion Club (peer consultation group)

Brain Training

Brain Friendly Working \& Writing

Career Perspectives

Efficient Writing Strategies

Adobe Indesign

Organisation of meetings, $\mathrm{PhD}$ courses or outreach activities

Participating in Fascination Of Plants Day 2017

Hosting the Plant Breeding Monday Seminars

Organising the SLU-WUR Plant Breeding Symposium 2019

7,9

5) Teaching \& Supervision Duties

Subtotal Personal Development

$\underline{c p}$

- Courses

Guest Lecture at HAN May 2018

Guest Lecture at HAN December 2018

22 May $2018 \quad 0,5$

Guest Lecture at HAN May 2019

10 Dec $2018 \quad 0,5$

Guest Lecture at HAN December 2019

21 May $2019 \quad 0,5$

9 Dec $2019 \quad 0,5$

- Supervision of BSc/MSc students

Supervising MSc student: Florian Tanner

2018

1,5

Supervising MSc student: Feije Sophie van Rijn

$2019 / 2020$

1,5

Subtotal Teaching \& Supervision Duties

Herewith the Graduate School declares that the PhD candidate has complied with the educational requirements set by the Educational Committee of EPS with a minimum total of 30 ECTS credits.

"A credit represents a normative study load of 28 hours of study. 
The research described in this thesis was funded through the Topconsortium voor Kennis en Innovatie (TKI) Tuinbouw \& Uitgangsmaterialen, with project number 1409-026, which is (partly) financed by the breeding companies Bejo Zaden, BASF, EastWest Seed, Hazera, Rijk Zwaan, Syngenta and Takii Seed.

Financial support from Wageningen University for the printing of this thesis is gratefully acknowledged.

Cover \& Layout design: Iliana Boshoven-Gkini | AgileColor.com

Printing: Ridderprint | Ridderprint.nl 


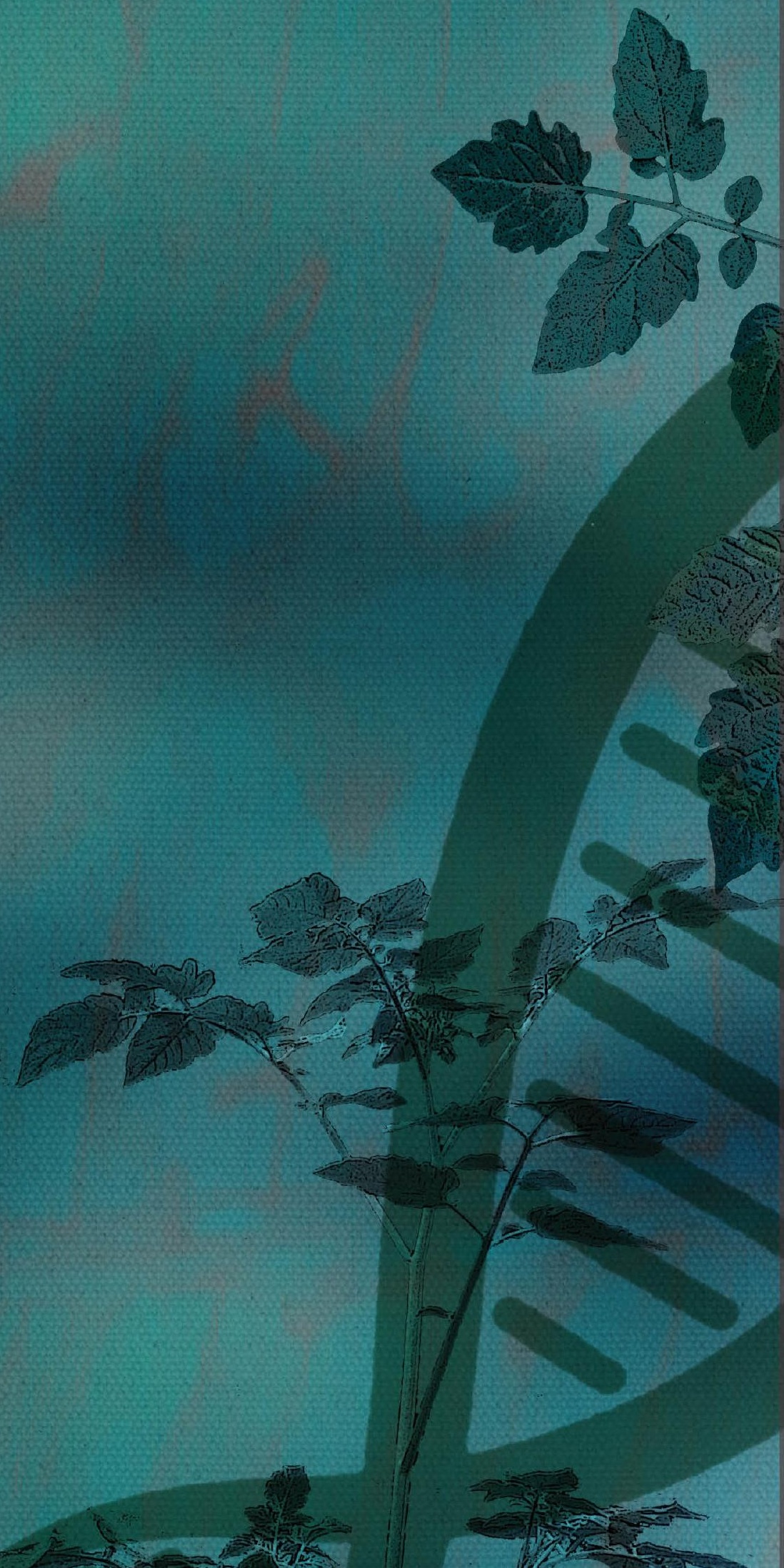

